\title{
Hanford Wells
}

\author{
V. L. McGhan \\ D. W. Damschen
}

May 1979

Prepared for the U.S. Department of Energy under Contract EY-76-C-06-1830

Pacific Northwest Laboratory Operated for the U.S. Department of Energy by Battelle Memorial Institute 


\title{
NOTICE
}

This report was prepared as an account of work sponsored by the United States Government. Neither the United States nor the Department of Energy, nor any of their emplayees, nor any of their contractors, subcontractors, or their employees, makes any warranty, express or implied, or assumes any legal liability or responsibility for the accuracy, completeness or usefulness of any information, apparatus, product or process disclosed, or represents that its use would not infringe privately owned rights.

The views, opinions and conclusions contained in this report are those of the contractor and do not necessarily represent those of the United States Government or the United States Department of Energy.

\author{
PACIFIC NORTHWEST LABORATORY \\ operated by \\ BATTELLE \\ for the \\ UNITED STATES DEPARTMENT OF ENERGY \\ Under Contract EY-76-C-06-1830
}

\author{
Printed in the United States of America \\ Available from \\ National Technical Information Service \\ United States Department of Commerce \\ 5285 Port Royal Road \\ Springfield, Virginia 22151
}

Price: Printed Copy \$__ Microfiche $\$ 3.00$

NTIS

-Pages Selling Price

\begin{tabular}{|c|c|}
\hline $001-025$ & $\$ 4.00$ \\
\hline $026-050$ & $\$ 4.50$ \\
\hline $051-075$ & $\$ 5.25$ \\
\hline $076-100$ & $\$ 6.00$ \\
\hline $101-125$ & $\$ 6.50$ \\
\hline $126-150$ & $\$ 7.25$ \\
\hline $151-175$ & $\$ 8.00$ \\
\hline $176-200$ & $\$ 9.00$ \\
\hline $201-225$ & $\$ 9.25$ \\
\hline $226-250$ & $\$ 9.50$ \\
\hline $251-275$ & $\$ 10.75$ \\
\hline $276-300$ & $\$ 11.00$ \\
\hline
\end{tabular}


PNL-2394

UC-1i

33679000533176

HANFORD WELLS

V. L. McGhan

D. W. Damschen

May 1979

Prepared for the U.S. Department of Energy Under Contract EY-76-C-06-1830

Pacific Northwest Laboratory

Richland, Washington 39352 


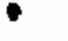

.

. 


\section{ACKNOWLEDGMENTS}

The authors wish to acknowledge the contribution made by G. L. Wagenaar and T. 3. Wood of the Orilling Technology Group, Rockwell Hanford Operations, in updating this document. 


\section{CONTENTS}

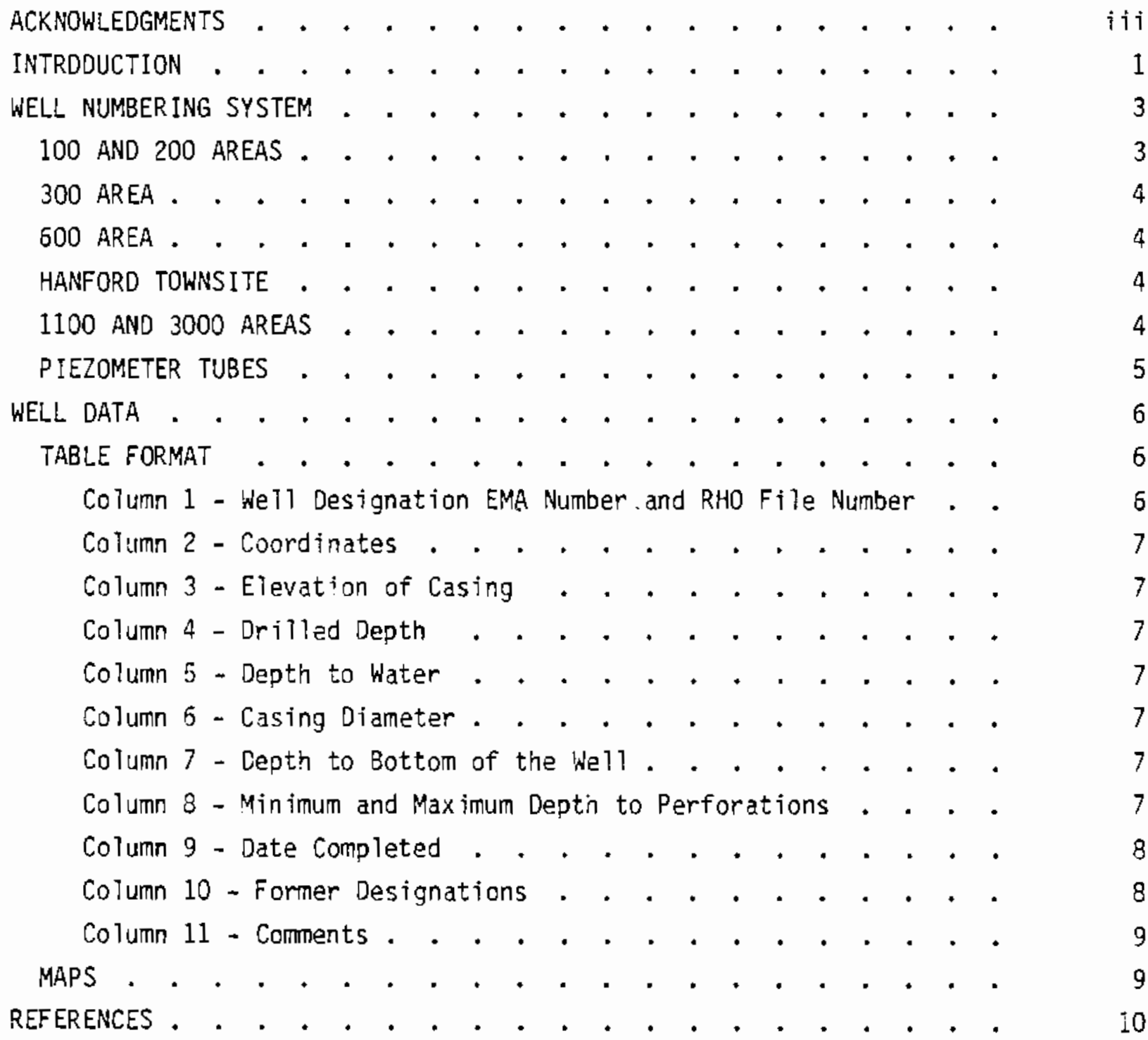




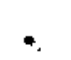

$\cdot$

,

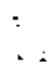

. 


\section{INTRODUCTION}

The Hanford Site contains over 2200 wells constructed from pre-Hanford Works days to the present. More than 200 older wells were mostly hand-dug farm wells, many of which are no longer usable or even visibie. The newer wells are cased wells which were either churn-drilled, drive-barrel-drilled or rotary-drilled. The wells drilled within the Hanford Site were constructed by contract drillers, by drilling crews in the employ of DOE prime contractors, or by the U.S. Geological Survey. Some of these were constructed originaily to provide sanitary water for outlying installations such as U.S. Army facilities, others to permit surveillance of the ground-water and sub-surface formations in the vicinity of waste disposal facilities, and still others to obtain data for a geological survey of the region. Some of these wells have been destroyed during construction of new facilities or through casing failures but are included on a master record to identify data obtained from them.

As of January 1979, over 1900 wells still exist (Table 1 contains a summary of status). About 850 of these existing wells were drilled to the groundwater table. About 780 of these wel?s (including about 24 farm welis) still contain water. The others have become dry through infiltration of sediments or a general lowering of the water table in their vicinity.

This report provides a complete documentation of weils in and adjacent to the Hanford Site. It supersedes all previous compilations of Hanford wells, the most recent being June 1977 (BNhl-2296). Not included in this report or the master record are those destroyed farm wells which were never surveyed into the Hanford coordinate system nor used for water-table measurements or water sampie collection. References 1,2 and 3 contain the available data on these wells.

While considerable effort has been made to obtain the most accurate and complete tabulation possible of the Hanford Site wells, inevitably some omissions and errors may exist, particularly for old farm wells. Periodic additions and corrections to this document will be issued to be made a part of this report. 
TABLE 1. Hanford We11s

Well Use

Amount

Ground-water Contamination Surveillance
Ground-water Hydrological Data Collection
Dry Wells for Monitoring Waste Management
Facilities

Basalt Stratigraphy Characterization

\begin{tabular}{|c|c|c|}
\hline Total Wells by Area & Constructed & $\begin{array}{l}\text { Existing } \\
\text { June } 1977\end{array}$ \\
\hline 100 Area & 116 & 63 \\
\hline 200 Area & 1328 & 1280 \\
\hline 300 Area & 37 & 30 \\
\hline 400 Area & 8 & 4 \\
\hline 600 Area & 664 & 518 \\
\hline 1100 Area (Richland) & 105 & 16 \\
\hline 300 Ared (N. Richland) & 8 & 6 \\
\hline $\begin{array}{l}\text { Existing Total Wells } \\
\text { by Site }\end{array}$ & 2266 & 1917 \\
\hline
\end{tabular}




\section{WELL NUMBERING SYSTEM}

Well structures are identified by the number 99 . The rest of the number designating a specific well is established mainly by its location on the plant. The designation includes three groups of symbols. The first group consists of the number 99 preceded by a number identifying the area in which the we 11 is located. Example: 199, 299, 399, 699, 1199, 3099.

\section{0 and 200 AREAS}

Wells located in the 100 and 200 Areas are further identified in the second group of symbols according to the designation of the particular area $(E, W, B, D, K, F, H, N)$, followed by the number of the sheet map encompassing that portion of the area in which the well is located. The sheet maps are shown on official second level maps for each area. Example: The first twosymbol groups for a well located within the area described by Sheet Map 24 of the 20D-E Area would be 299-E24 $\rightarrow$. The 100-K Area and the 100-N Area second level maps are not further divided into sheet maps so the second group of symbols for these areas is simply " $K$ " and "N". The third-symbol group in the well number identifies the specific structure with in the sheet map area. In some cases the numbering system is arbitrary; but where practical, numbers were chosen in accordance with a provious numbering system. For example, the well formerly designated 361-B- $\underline{6}$ now has the number 299-E28- 6 .

Some of the monitoring we 115 in the 200 Areas are dry wells, i.e., wells that do not extend to the water table. These have been differentiated from deeper wells by numbering all of the shallow wells with the third-group numbers greater than 50. In some cases we 175 have been designated with 100 and 200 Area numbers even though they are actually located outside the area fence. These structures are monitoring wells adjacent to ground disposal facilities that are located outside the area. Monitoring wells adjacent to the $\mathrm{BC}$-Cribs are exampies of this situation. 
300 AREA

Wells in the 300 Area are designated in a manner similar to that described for the 100 and 200 Areas. The single difference occurs in the second symbol group in which no area designation is given, but which consists simply of the 300 Area sheet map number.

\section{AREA}

The 600 Area includes all of the Hanford Site outside the limited access areas. Well numbers in this area are prefixed by 699. The second and third groups of numbers for 600 Area we17s consist of the north and west plant coordinates, respectively, rounded off to the nearest $1000 \mathrm{ft}$. For example, a we 11 located at plant coordinates, W14554 would be designated 699-26-15. If the well is located south or east of the plant coordinate origin, an $S$ or $E$ is used with the appropriate number. Some 600 Area wells are located within $1000 \mathrm{ft}$ of each other; these have letters (A, B, C, etc.) following the numbers for unique identification. A few wells located outside of the Hanford Site boundaries have also been given 600 Area designations.

\section{AND 3000 AREA}

Well numbers in the 1100 Area (City of Richland) and the 3000 Area (old North Richland) have 1199 and 3099 prefixes, respectively. The second and third number groups are the Richland coordinates rounded to the nearest $1000 \mathrm{ft}$. These wells were located in the Richland coordinate system because the plant grid had not been extended this far south when they were surveyed. New wells within the Hanford Site boundaries in the 1100 and 3000 Areas are located and named in the 600 Area plant grid system. However, wells drilled since 1961 within the Richland city limits are not included in the listing. 


\section{PIEZOMETER TUBES}

Piezometer tubes were installed in 85 cased we11s to measure potentials and sample the aquifer at various depths. Presence of these tubes is indicated by a letter ( $0, P, Q, R, S$, or $T)$ after the well number; these tubes are listed with the corresponding well in this tabulation. Six other sets of piezometer tubes are individually drilled several feet apart, and are also designated by the sequence of letters $P, Q, R$, etc. Each tube of this type is regarded as a separate well in the tabulation. 


\section{WELL DATA}

The tabulation of existing wells and others for which data are recorded is composed of sections, each of which reports wells from one of the areas. This tabulation is given at the end of this report. It was prepared from computer listings of the master well information file maintained by PNL Water and Land Resources Department.

\section{IABLE FORMAT}

The table format consists of the columns described below. Column 1 - Well Designation EMA Number and RHO File Number

All of the existing wells that can be located and others for which data have been reported are designated in Column 1 by the system described earlier. Well designations can change when wells are surveyed into a coordinate system or when a resurvey is made.

The EMA (Environmental Monitoring Analys is Accumulation and Reporting) number in this column identifies the well for the computer program which processes sampiing schedules and results. All wells assigned an EMA number have been or are presently in the routine ground-water sampling schedule.

- The RHO File numbers in this column pertain to tank farm wells. The first number is the tank farm number; the second number is the tank number; and the third number is the position of the we 11 around the tank. Each tank is treated as a clock face with numbers located in hour quadrants. Numbers may run 1-24 on a 24-hr basis. Twelve o'clock is due north on tank center line. Wells outside the tank area are sequenced in like manners with the center line of the farm due north -12 o'clock.

A series of asterisks indicates that a well (or wells) has been removed from the active wells list. These wells may have been filled in, abandoned or otherwise destroyed.

A single asterisk indicates that the preceding well had piezometer tubes which have since been removed. In cases where only selected piezometer tubes were removed, they are noted by the single asterisk. 
Column 2 - Coordinates

Well coordinates are listed in Column 2 when this information is available. Plant coordinates are designated "P", Richland coordinates "R". For certain wells the coordinates have been estimated.

Column 3 - Elevation of Casing

The elevation of the top of the well casing is reported in feet above mean sea level. Some values have been estimated.

Column 4-Drilled Depth

The depth of the we11, as determined at the time the well was completed, is listed in feet below the top of the casing.

Column 5 - Depth to Water

The depth from the top of the casing to the water table is listed in feet for wells in which this distance has been measured. These depths to water can fluctuate over several feet, particularly for wells near the Columbia River. Severa? old farm wells which are not currently being used for water-table elevation measurements are designated "wet" or "dry" as appropriate. In addition, numerous wells used by Washington Public Power Supply System (WPPSS) as seismic shot holes are listed as "dry".

Column 6 - Casing Diameter

The diameter of the casing used in construction of the well is given in inches. A few of the weils constructed at Hanford required casing of more than one size, and for these only the diameter of the access hoie is recorded. The length of the shortest side is listed for rectangularly shaped farm wells.

Coiumn 7 - Depth to Bottom of the Well

The most recent measurement of the bottom depth of the we 11 is reported. Generally, wells become partially filled as sediments enter the well through the perforations. The depths shown in this column were determined from data obtained since 1978 . 
Column 8-Minimum and Maximum Depth to Perforations

The depths to the top and bottom of the well casing perforations are expressed in feet below the top of the well casing. However, in some wells aiternating perforated and unperforated sections may be between the depths 1isted. Wells that were completed with well screens have no perforation depths 1isted. The screen depths are listed under the comments column.

Column 9 - Date Completed

This column records the date, to the nearest month where possible, on which the well was completed. In some cases only the year in which the well was drilled is known.

Column 10 - Former Designations

Column 10 lists the designations that have been used to identify the well in the past. These include local Hanford names or names applied by the agency responsible for constructing the we11, such as the USGS designations.

The reference numbers used in this column refer to the reference listing for this report. The following is a list of other abbreviations used in the former designations column:

$\begin{array}{ll}\text { DH } & \text { Drill Hole } \\ \text { FFTF } & \text { Fast Flux Test Facility } \\ \text { HD } & \text { Hanford Durand (driller's name) } \\ \text { HI } & \text { Hanford International (driller's name) } \\ \text { HR } & \text { Hanford Ranney (driller's name) } \\ \text { N. Run } & \text { North Run (well taping and sampling route) } \\ \text { RD } & \text { Richland Durand } \\ \text { RI } & \text { Richland International } \\ \text { RRY } & \text { Railroad yard } \\ \text { Sea. In . Emp. } & \text { Seattle Inland Empire Gas Co. } \\ \text { TH } & \text { Test Hole } \\ \text { USGS } & \text { U.S. Geological Survey } \\ \text { WBD } & \text { White Bluffs Durand } \\ \text { WPPSS } & \text { Washington Public Power Supply System }\end{array}$


WWOG

$\times$ W

HAN
Western Washington $0 i 1$ and Gas Co.

We 11 number $x$

Former Hanford Townsite

\section{Column 11 - Comments}

Noted in this column are: changes made to the wells during routine maintenance, sampling pump installation, seismic study wells, pumped water supply welis, and welis which have had screens installed. Wells that were not located during the census of wells taken for this report are so indicated in this column. Many of these wells have probably been destroyed.

MAPS

Maps of the 200 East and 200 West Areas are included on the following pages, and a 600 Area map is in the binder. Only the wells which extend to ground water are shown in the 200 Area maps. The 600 Area maps shows all wells currently available for water level measurements and ground-water sampling. Area maps showing well locations are available for the the 100 N Area $(H-1-38646)$ and the 300 Area $(H-3-32585)$. 


\section{REFERENCES}

1. Newcomb, R. C., J. R. Strand and F. J. Frank. 1972. Geology and Groundwater Characteristic of the Hanford Reservation on the U.S. Atomic Energy Commission Washington. Geol. Survey Prof. Paper 717.

2. Jenkins, O. P. 1922. Underground Water Supply of the Region About White Bluffs and Hanford. Washington Department of Conservation and Development, Division of Geology, Bulletin No. 26.

3. Walters, K. L. and M. J. Grolier. 1960. Geology and Groundwater Resources of the Columbia Basin Project Area, Washington. State of Washington Water Supp Ty Bullet in No. 8, Vol. 1. 


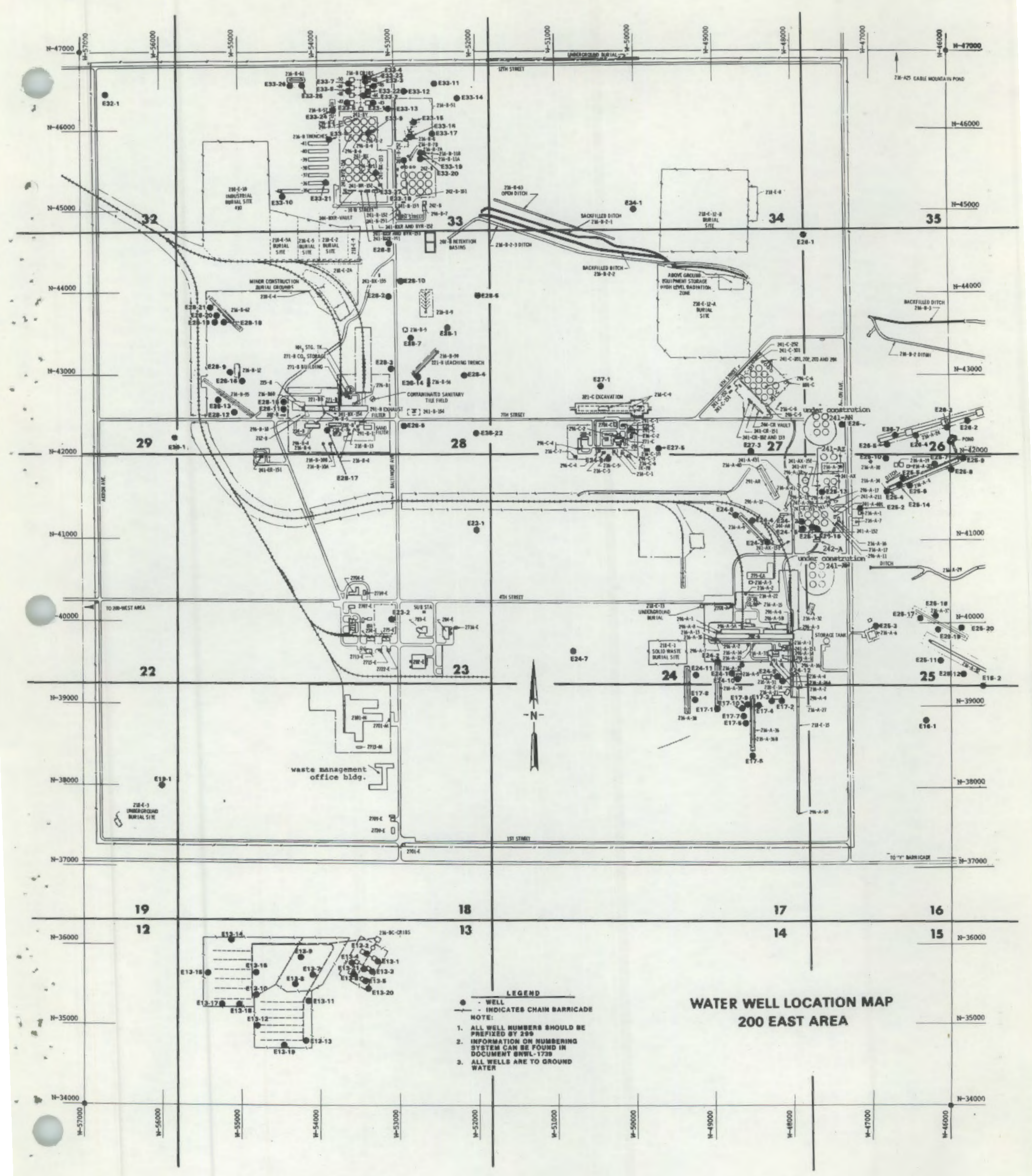




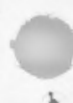

1

$+5$

,

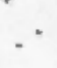

.

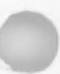

$\therefore$

.

$\because$

4

0 


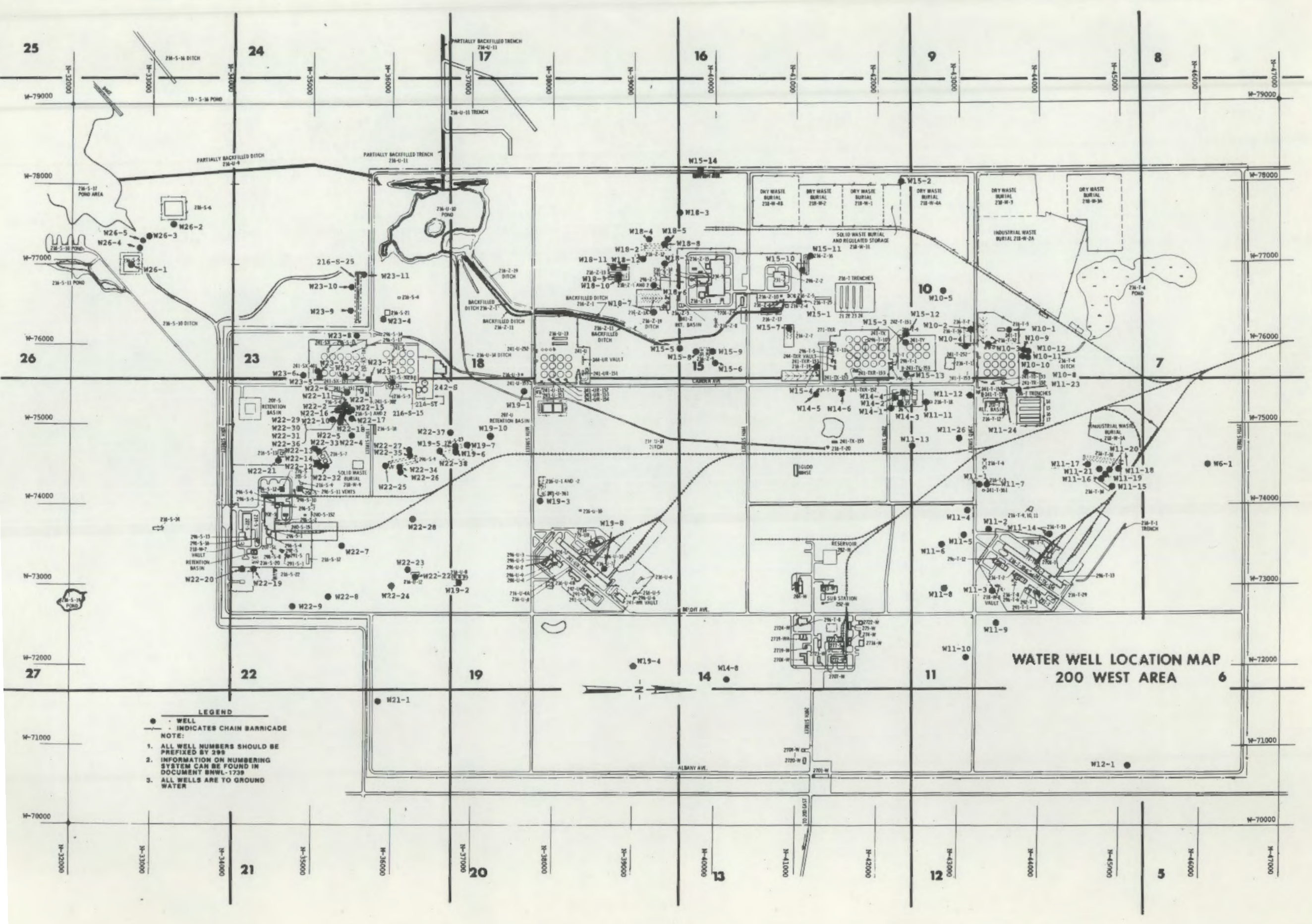




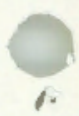

1

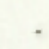

,

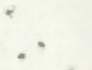

.

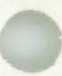

$\therefore$
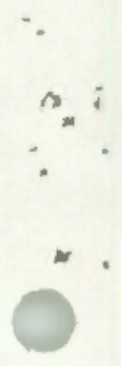


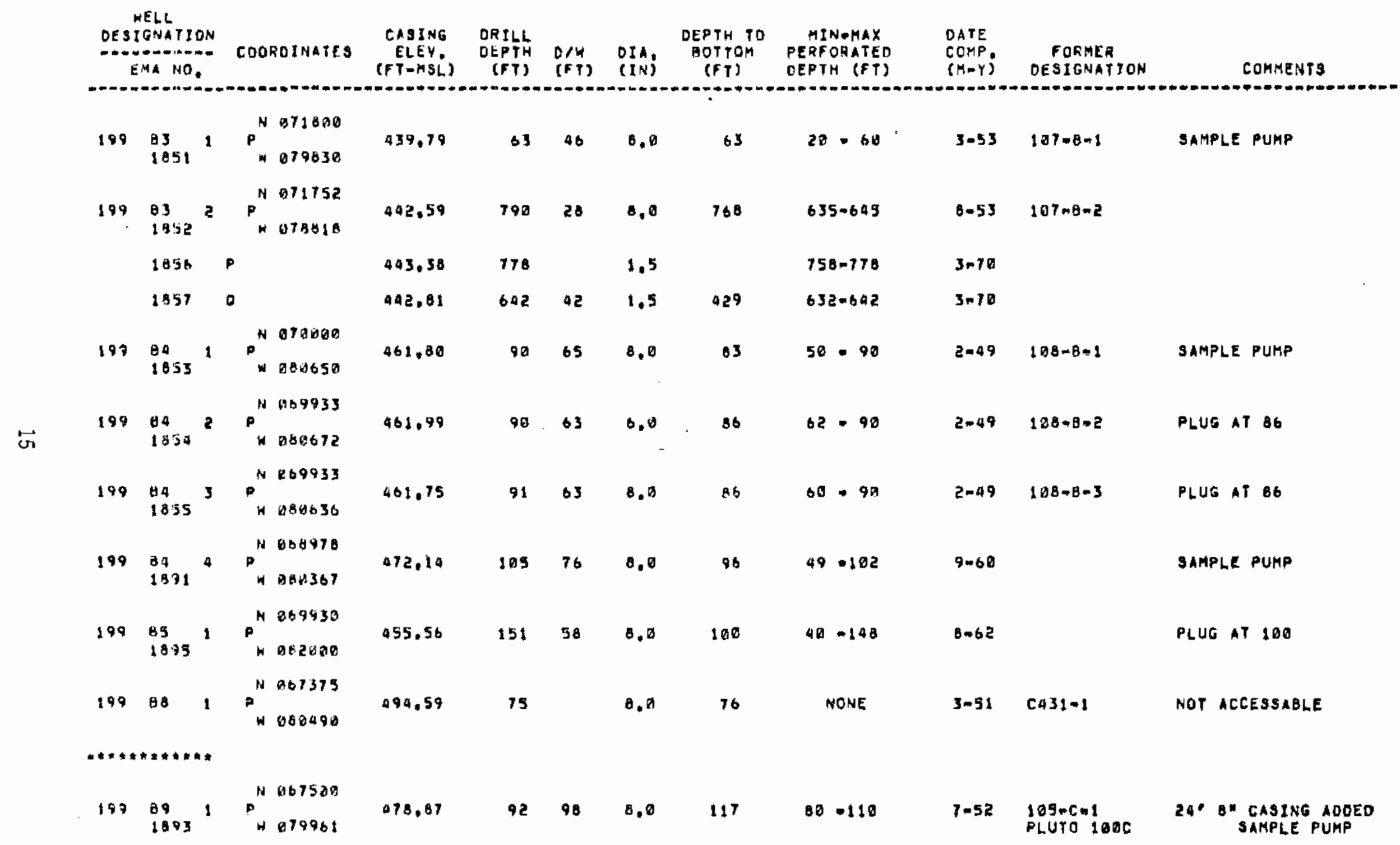


HELL

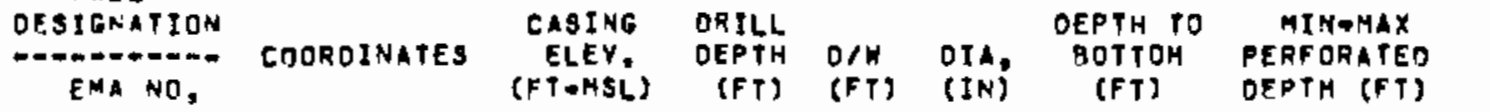

DATE

COMP, FORMER

(M-Y) OESIGNATION

COHMENTS

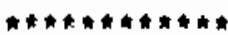

$199025 P^{N 090783}$ $1894 N 052030$

460.87

$95 \quad 73 \quad 6,0 \quad 99$

6.60

OAMPLE PUHP

占

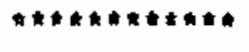

$199 l_{1092}^{12} P^{N 092125}$

469.67

9169

0.8

91

$35 \cdot 90$

$\theta 00$

SAHPLE PUMP

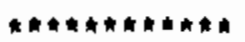

$\begin{array}{llll}199 & 09 & 2 & P^{N} 090725 \\ 1861 & & & \\ 199 & \begin{array}{l}03 \\ 1362\end{array} & P^{N} 094720 \\ 4052205\end{array}$

444.61

77 DRY

6,0

44

$50-79$

$6=5$

$107-0.1$

909.06

$61 \quad b 2$

6.0

81

$35 \cdot 79$

$6-52 \quad 107-0-2$

SAHPLE PUHP 


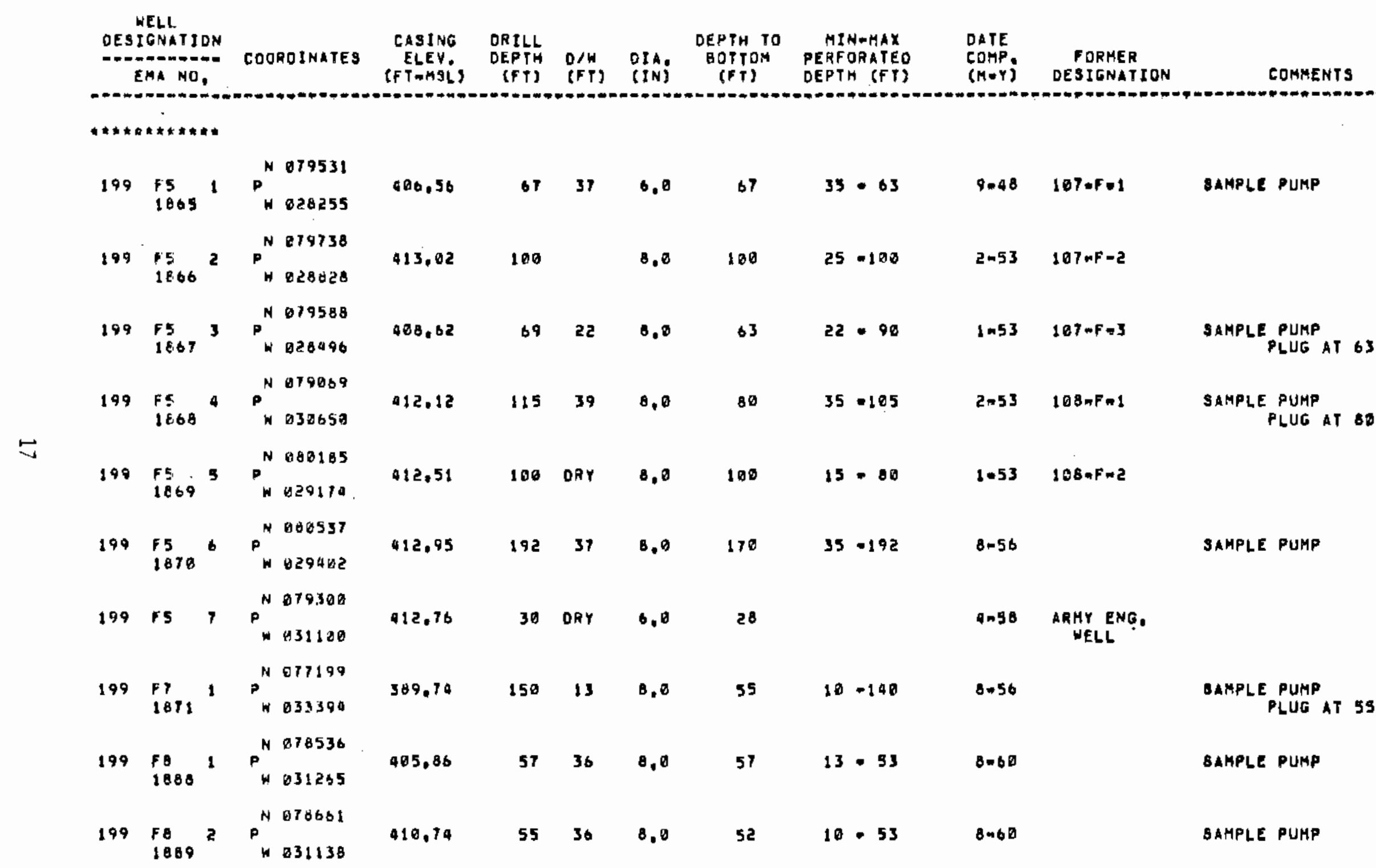




\begin{tabular}{|c|c|c|c|c|c|c|c|c|c|c|c|}
\hline OESI & $\begin{array}{l}\text { ELL } \\
\text { GNATIDN } \\
\text { MA NOE. }\end{array}$ & COORDINATES & $\begin{array}{c}\text { CASINO } \\
\text { ELEY } \\
\text { (FT-MSL) }\end{array}$ & $\begin{array}{l}\text { DRTLL } \\
\text { DEPTH } \\
\text { (FT) }\end{array}$ & 足 & DIA; & $\begin{array}{l}\text { OEPTH TO } \\
\text { BOTTOM } \\
\text { (FT) }\end{array}$ & $\begin{array}{l}\text { MIN+MAX } \\
\text { PERFORATEO } \\
\text { OEPTH (FT) }\end{array}$ & $\begin{array}{l}\text { DATE } \\
\text { COMP; } \\
\text { (MNY) }\end{array}$ & $\begin{array}{l}\text { FORMER } \\
\text { DESIGNATION }\end{array}$ & COMAENTS \\
\hline 199 & ${ }_{1890^{43}}^{1}$ & $\begin{array}{l}F_{N}^{N} 094994 \\
N 040052\end{array}$ & 421,98 & 15 & as & 0,0 & 75 & $29 \cdot 74$ & $8=60$ & & SAMPLE PUMP \\
\hline 196 & $\begin{array}{l}1442 \\
1876^{2}\end{array}$ & $P_{N 038565}^{N 095209}$ & 417,43 & 386 & 3 & 6,0 & 311 & $29 \cdot 58$ & $5-52$ & $807-\mathrm{H}+2$ & CONF INED AQUSFER \\
\hline 199 & $\begin{array}{l}\mathrm{HA}, 3 \\
1877^{3}\end{array}$ & & & 53 & 30 & 6.0 & & $34 \cdot 55$ & $5-74$ & & SAMPLE PUMP \\
\hline
\end{tabular}




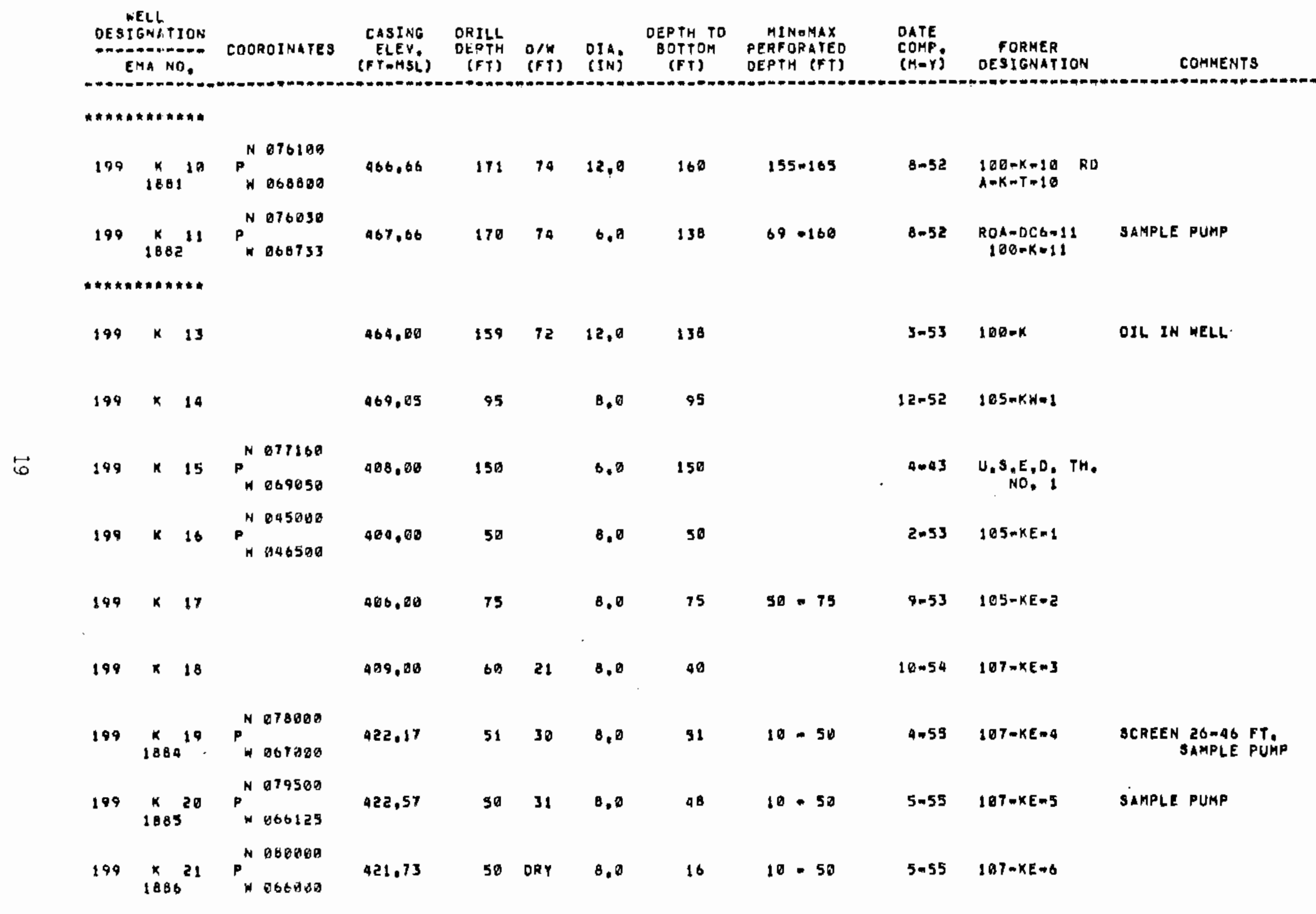




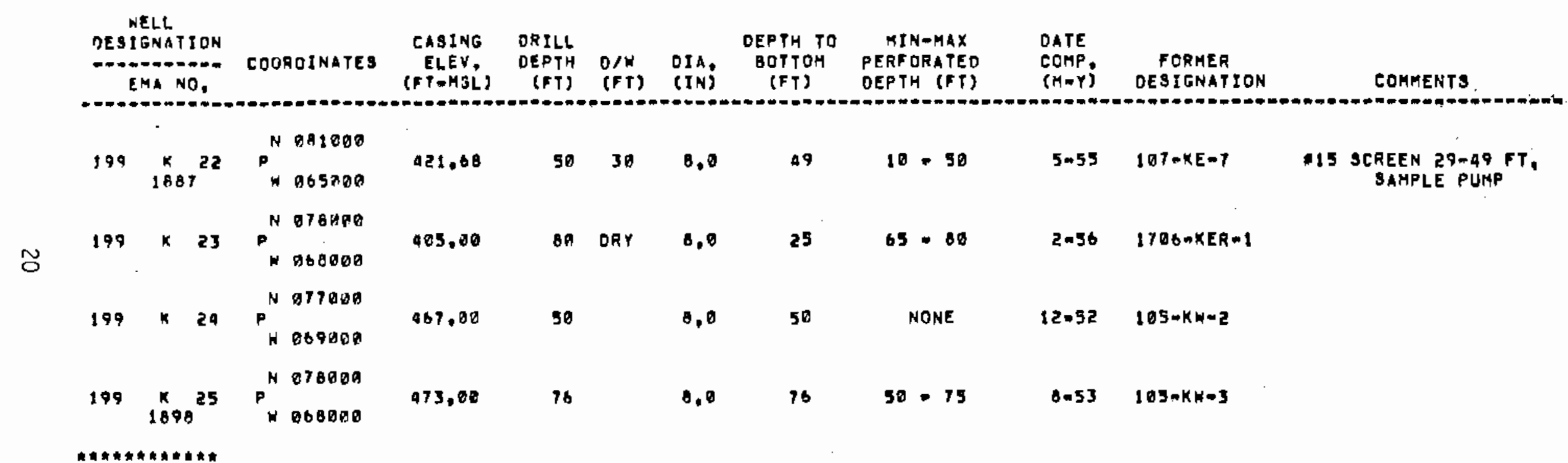




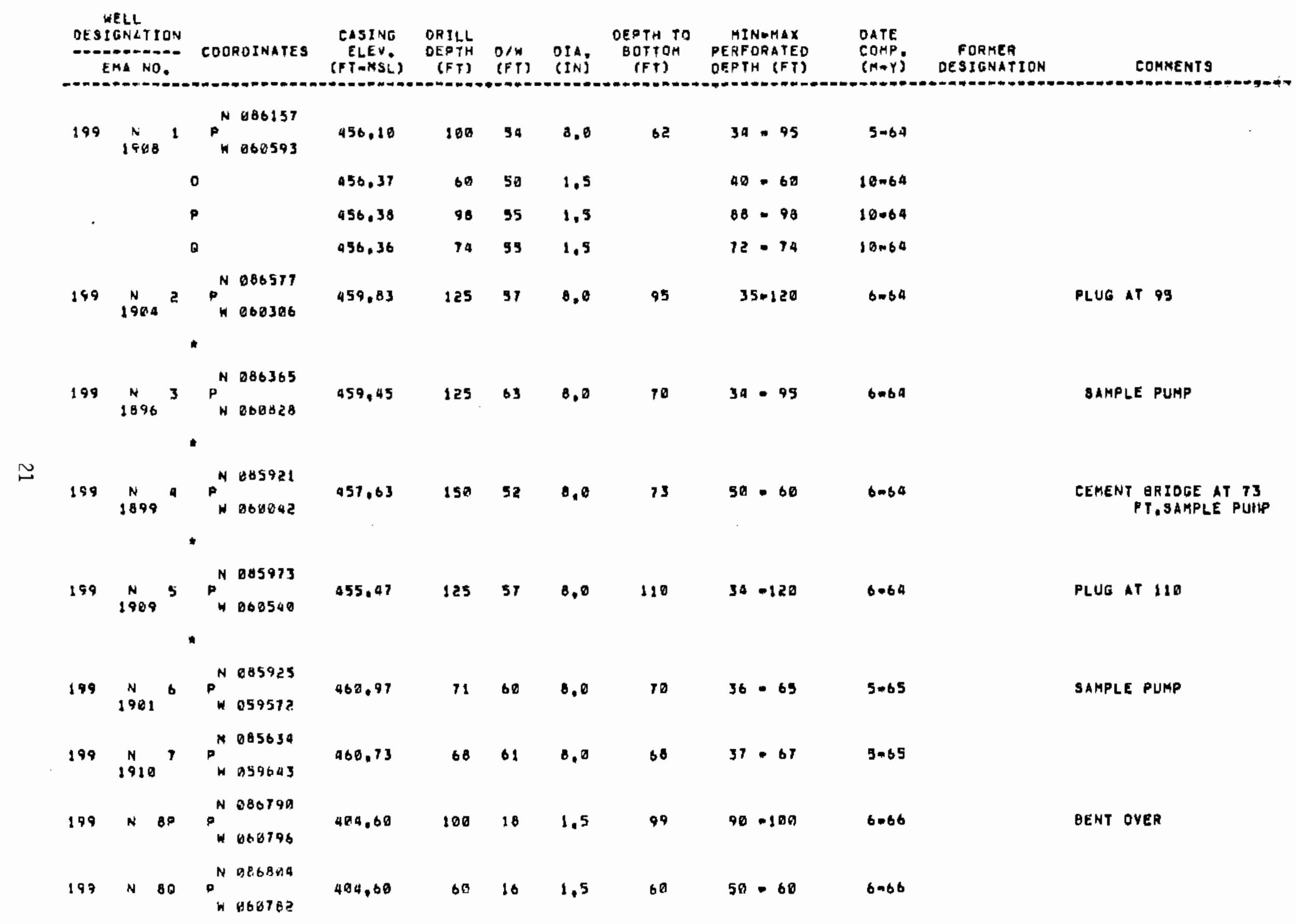




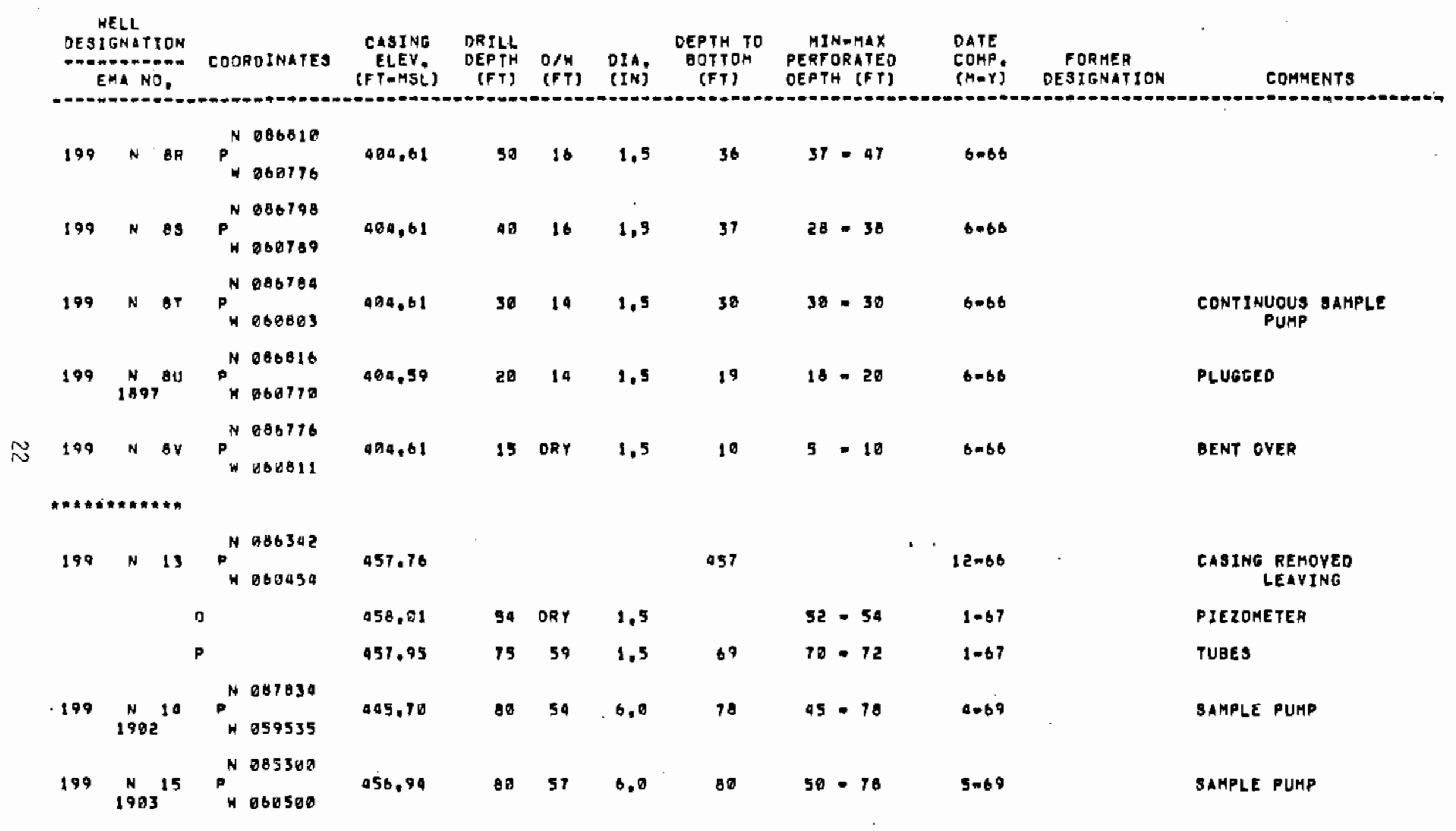




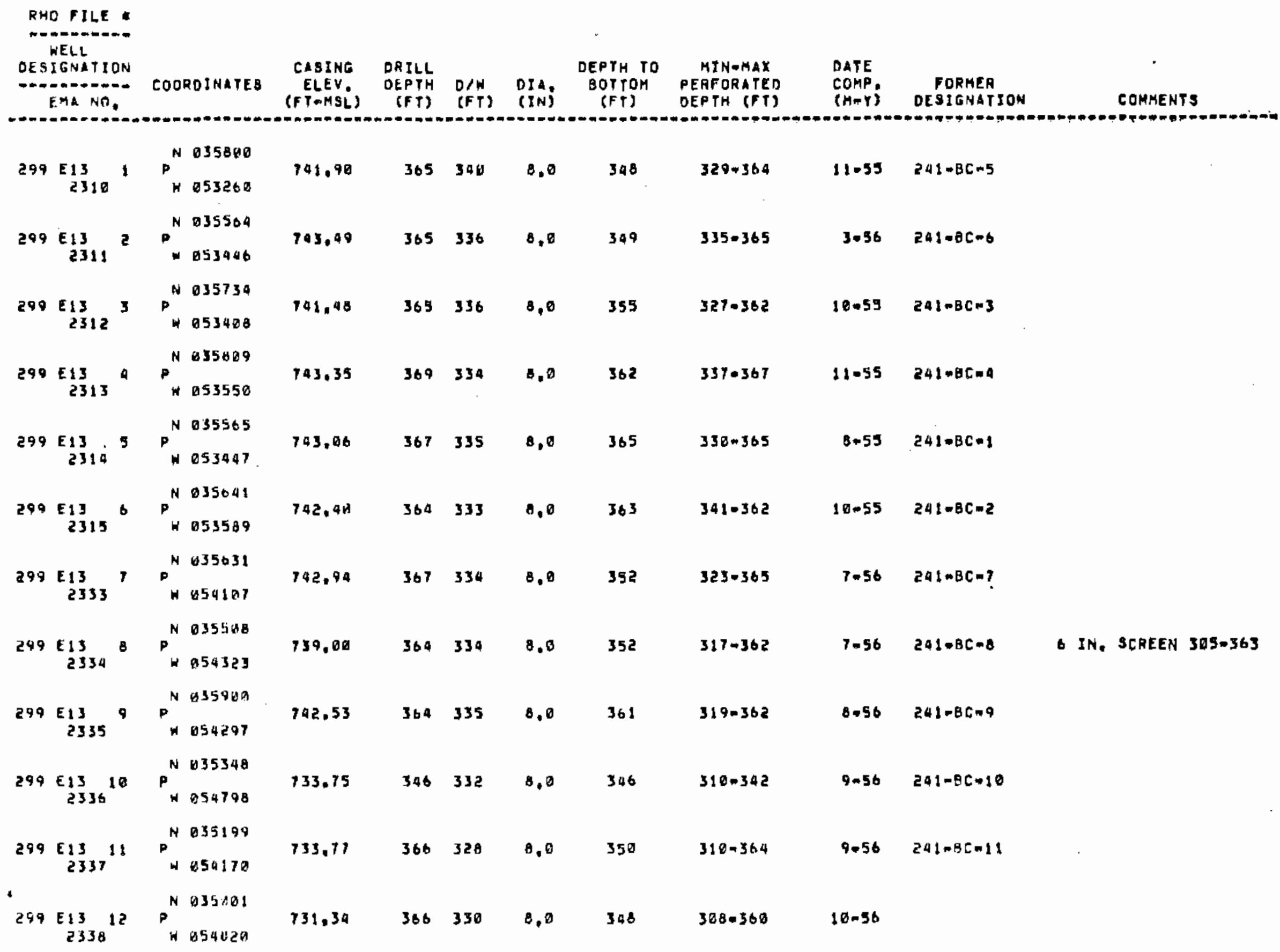




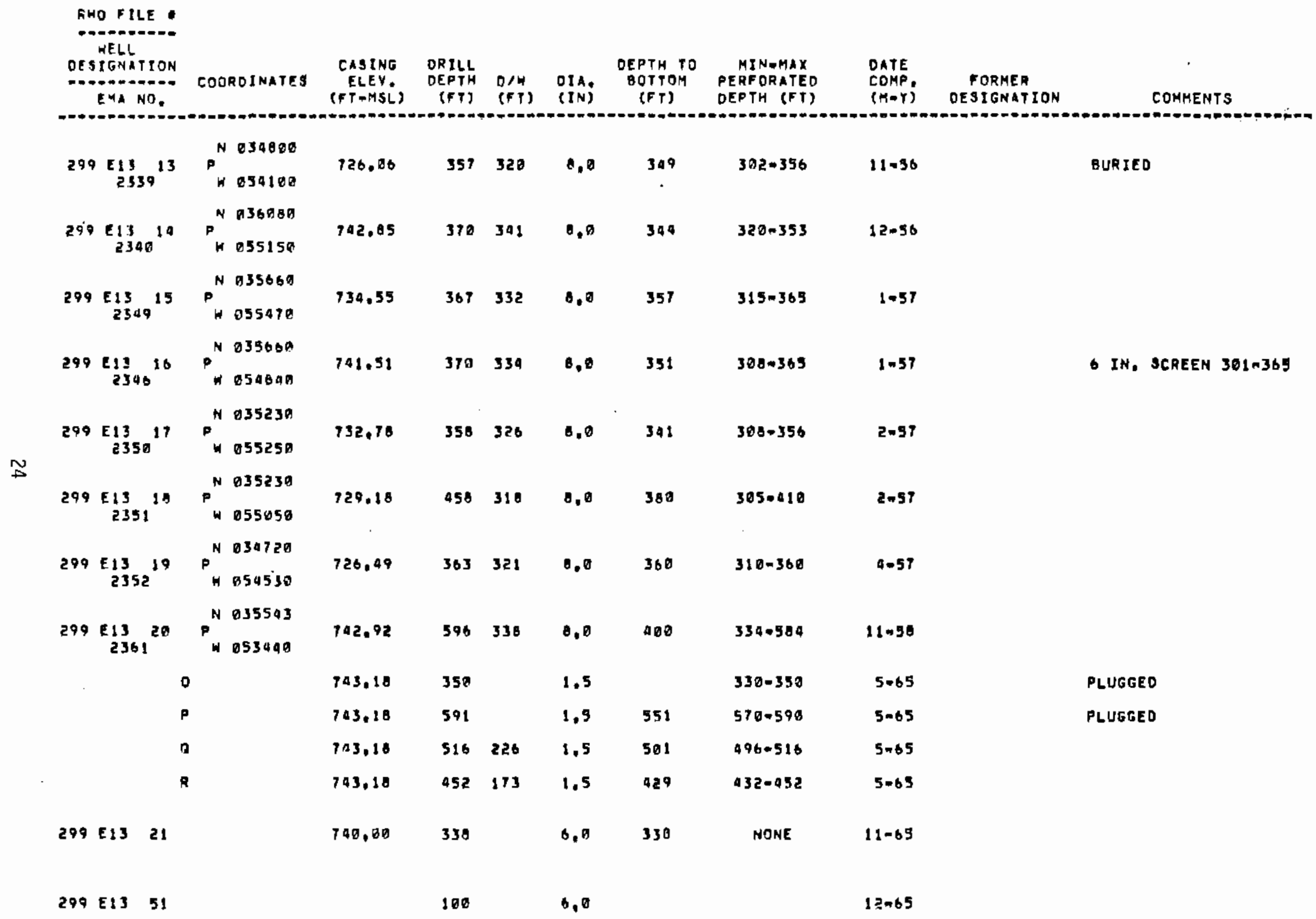


RMO FILE

RHD FILE

$$
\text { NELL }
$$

DESIGNATION EMA NO,

CODROINATES

$\begin{array}{lll}\text { EASING DRILL } & & \text { DEPTH YO MINMHAX } \\ \text { ELEY. DEPTH O/H OTA, BOTTOM PERFORATED }\end{array}$ BOTTOM PERFDRATED DEPTH (FT)

DATE

COMP. FORMER

(M-Y) DESTENATION

CORMENTS

$299 E 13 \quad 52$

9a

6,0

$12 \div 65$

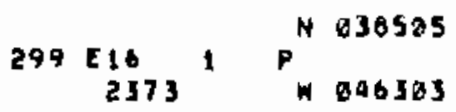

$696.94 \quad 038$

$8.0 \quad 278$

$280-455$

$1=61$

0

696,20 308 ORY

270

$4-84$

$\$ 96.37 \quad 476 \quad 290$

$1.5 \quad 474$

451.476

$10-62$

2002 0

$2903 R$

$690.37 \quad 417 \quad 292$

$1,5 \quad 398$

$392=417$

$10-62$

696.80

$364 \quad 295$

$1.5 \quad 336$

336.363

$10-62$

in

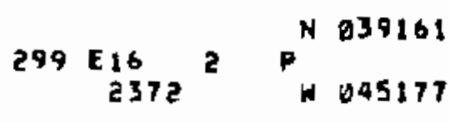

$689.56 \quad 340 \quad 200 \quad 8.0 \quad 33$

$265 \cdot 336$

$12-60$

$299-E 25=13$

$209 E 17, P_{232 B}^{N 059053}$

718.88

$336 \quad 318$

0.0330

$363 \times 333$

$12-59 \quad 216-1-10-1$

$299 E 172 P^{N 039003}$

$715.74 \quad 406 \quad 313 \quad 0.8$

$303-398$

$4=60$

$299 E 17$ 2368 $P_{N 043590}^{N 034866}$

715.83

$400 \quad 31$

0.0398

3180000

$3+60$

$299 E 17 \quad 4 \quad P^{N 839999}$

717.26

$400 \quad 312$

8.0366

$298-398$

9.35

$299517,5 P^{N 511} P^{N 038700}$

718,65

33631

8.8

298.335

$7=65$

$299 \varepsilon 176 P^{N} 036190$

720.17

$500 \quad 316$

$8,0 \quad 099 \quad 3009460$

$6-65$

$2996177 P^{N 320}+238710$

$400 \quad 314$

$0.0 \quad 384$

$300 \cdot 385$

$4=66$ 


\begin{tabular}{|c|c|c|c|c|c|c|c|c|c|c|c|c|}
\hline $\begin{array}{r}\text { DE } 55^{\prime} \\
=8 \\
E\end{array}$ & $\begin{array}{l}\text { KELL } \\
\text { ISNATIO } \\
\text { YMA NO }\end{array}$ & & COOROINATES & $\begin{array}{c}\text { CAOING } \\
\text { ELEV } \\
\text { (FI-MSL) }\end{array}$ & $\begin{array}{l}\text { DRILL } \\
\text { OEPYH } \\
\text { (FT) }\end{array}$ & $\begin{array}{l}\text { D/H } \\
(F T)\end{array}$ & DIA & $\begin{array}{l}\text { DEPTH TO } \\
\text { BOTTOH } \\
\text { (FT) }\end{array}$ & $\begin{array}{l}\text { RINAHAX } \\
\text { PERFORATED } \\
\text { OEPTH (FT) }\end{array}$ & $\begin{array}{l}\text { DATE } \\
\text { COMP, } \\
\text { (MNY) }\end{array}$ & $\begin{array}{l}\text { FORMER } \\
\text { DESIGNATION }\end{array}$ & COMHENTS \\
\hline 299 & $\begin{array}{l}E 17 \\
2513\end{array}$ & - & $\begin{array}{l}\text { P } \\
+039121 \\
W 04247\end{array}$ & 718.73 & 365 & 314 & 6.0 & 362 & $303-362$ & 8.67 & & \\
\hline 299 & E17 & 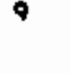 & & 717,98 & 325 & 313 & 6.0 & 321 & $3: 0 \cdot 320$ & $5=68$ & & \\
\hline 298 & $\begin{array}{l}E 17 \\
2515^{2}\end{array}$ & 19 & & 719.62 & 325 & 319 & & 323 & $310-320$ & $6 m 68$ & & \\
\hline 299 & E17 $2516^{5}$ & $5 \pi$ & & & 99 & & 6.0 & & NONE & $1=66$ & & \\
\hline 299 & $\begin{array}{l}199 \\
2359\end{array}$ & 1 & P 156023. & 736.00 & 370 & 334 & 8,0 & 336 & 320.370 & 12.37 & OH+17 & $\begin{array}{l}4 \text { IN LiNER TO } 371 \mathrm{FT}^{\mathrm{T}} \\
\text { DEEPENEO } 5=77\end{array}$ \\
\hline 299 & $\begin{array}{l}E 23 \\
2208\end{array}$ & 1 & ${ }^{P} W 052000$ & $7 A 9,65$ & 340 & 308 & 8.8 & 337 & 310.300 & $4-48$ & $361-8-8$ & \\
\hline \multirow[t]{4}{*}{299} & $\begin{array}{l}E 23 \\
2376\end{array}$ & 2 & P & 721.08 & 455 & 320 & $B .0$ & 438 & $304-435$ & $9=61$ & - & \\
\hline & & ] & & 721.26 & 340 & 315 & 1.5 & & $320-340$ & $5=65$ & & \\
\hline & 2406 & $\mathbf{p}$ & & 721,26 & 456 & 318 & 1.5 & 453 & $436-436$ & $5-65$ & & \\
\hline & 2007 & D & & 721.26 & 400 & 318 & 1.5 & & $380-980$ & $5-65$ & & \\
\hline 299 & $\begin{array}{l}\text { E } 24 \\
2317\end{array}$ & 1 & $P_{H}^{N} 839396863$ & 915,89 & 343 & 314 & 8.0 & 301 & $300-341$ & 3.55 & $216-4-5-12$ & \\
\hline 299 & $\begin{array}{l}\text { E24 } \\
2329\end{array}$ & 2 & $P^{N} 039084$ & $T 17,19$ & 350 & 312 & 8.0 & 303 & $245+348$ & 6.56 & $216-A-10=4$ & \\
\hline 299 & $\begin{array}{l}E 20 \\
2345\end{array}$ & 3 & P & 698.69 & 333 & & 8.0 & 250 & $277 \cdot 331$ & $6=56$ & $216-1-9.1$ & OBSTRUCTIDN AT 253FT \\
\hline & . & : & 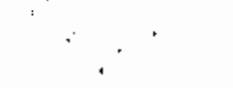 & $\because$ & & & & & & & $\therefore$ & s \\
\hline
\end{tabular}




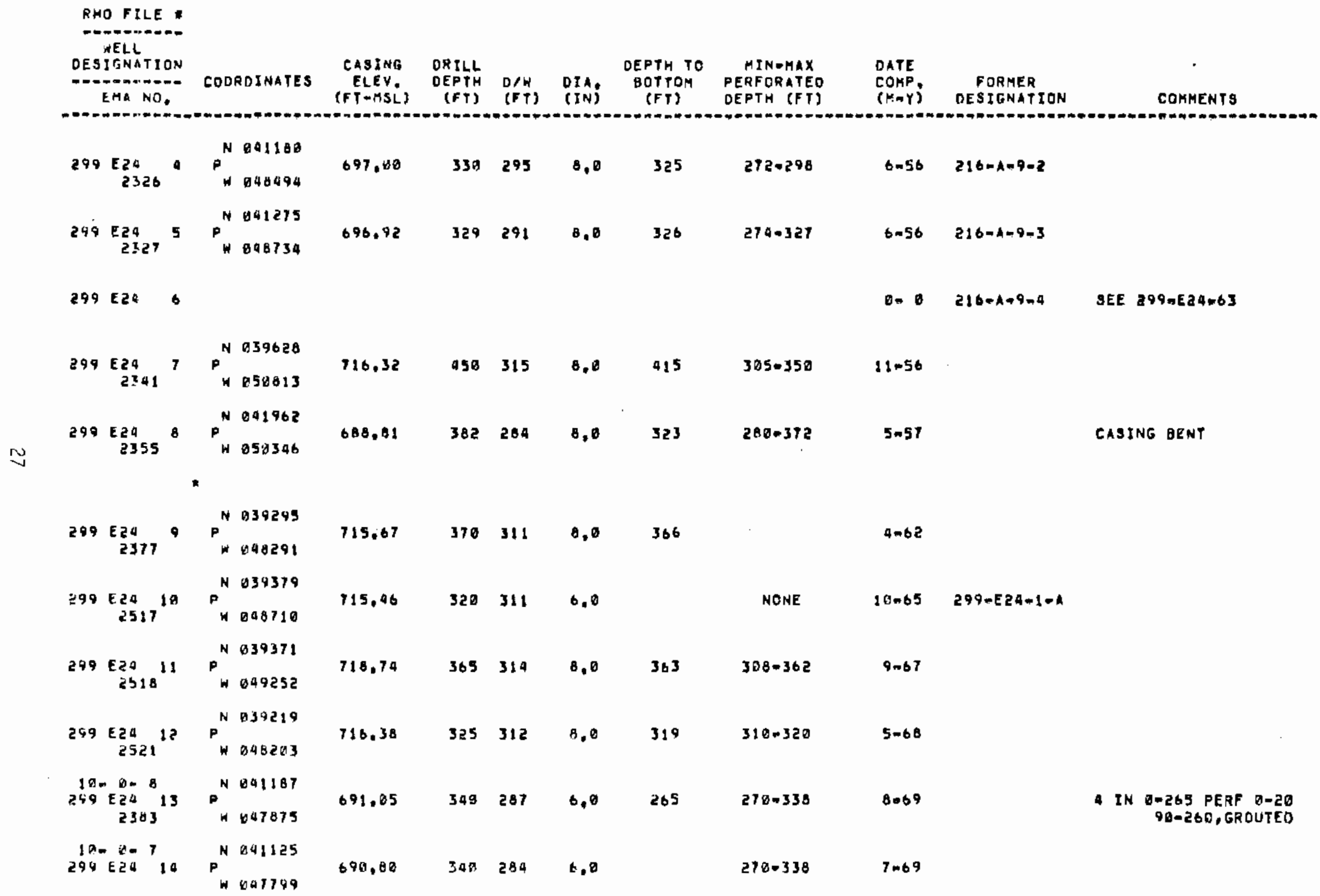




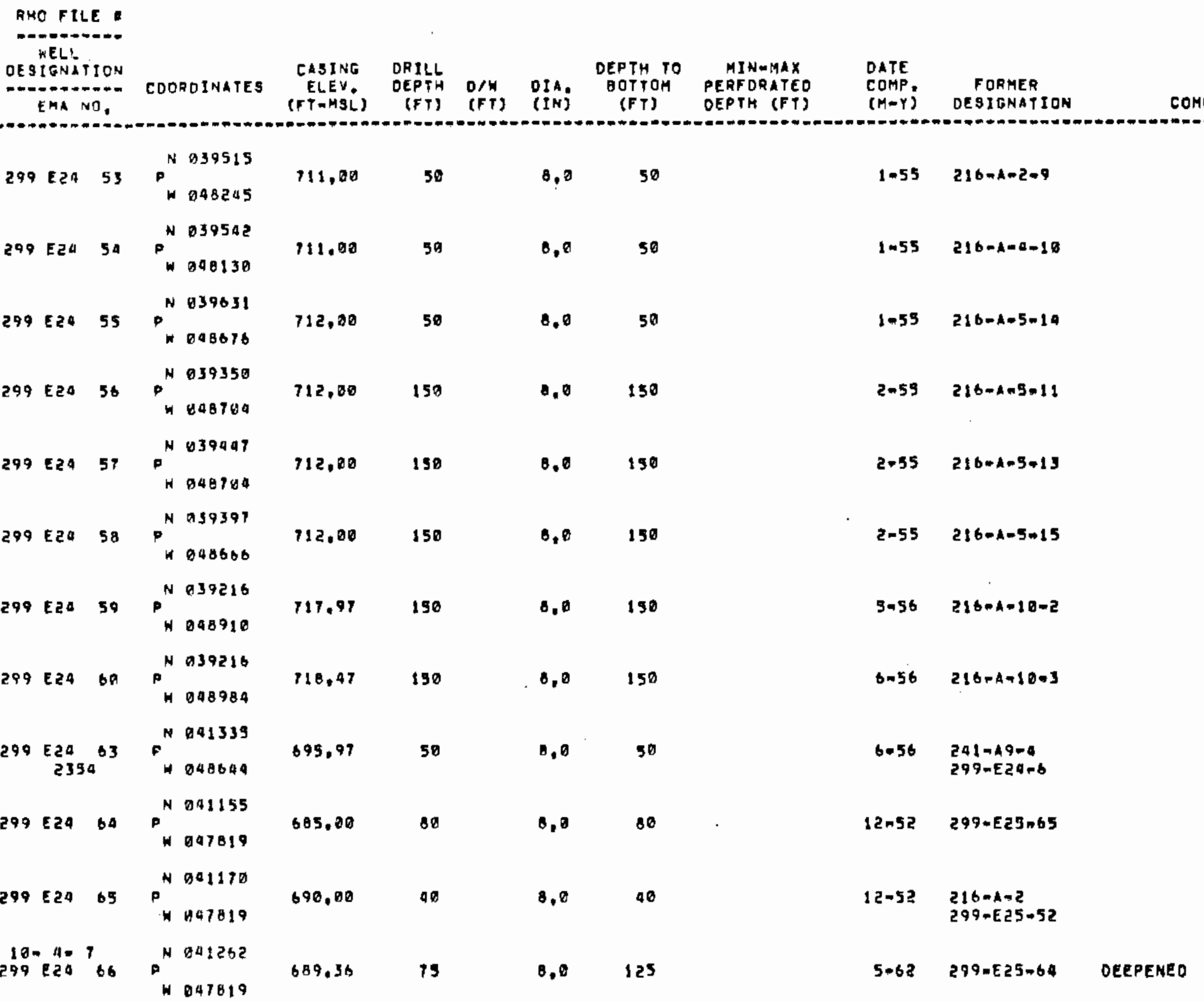




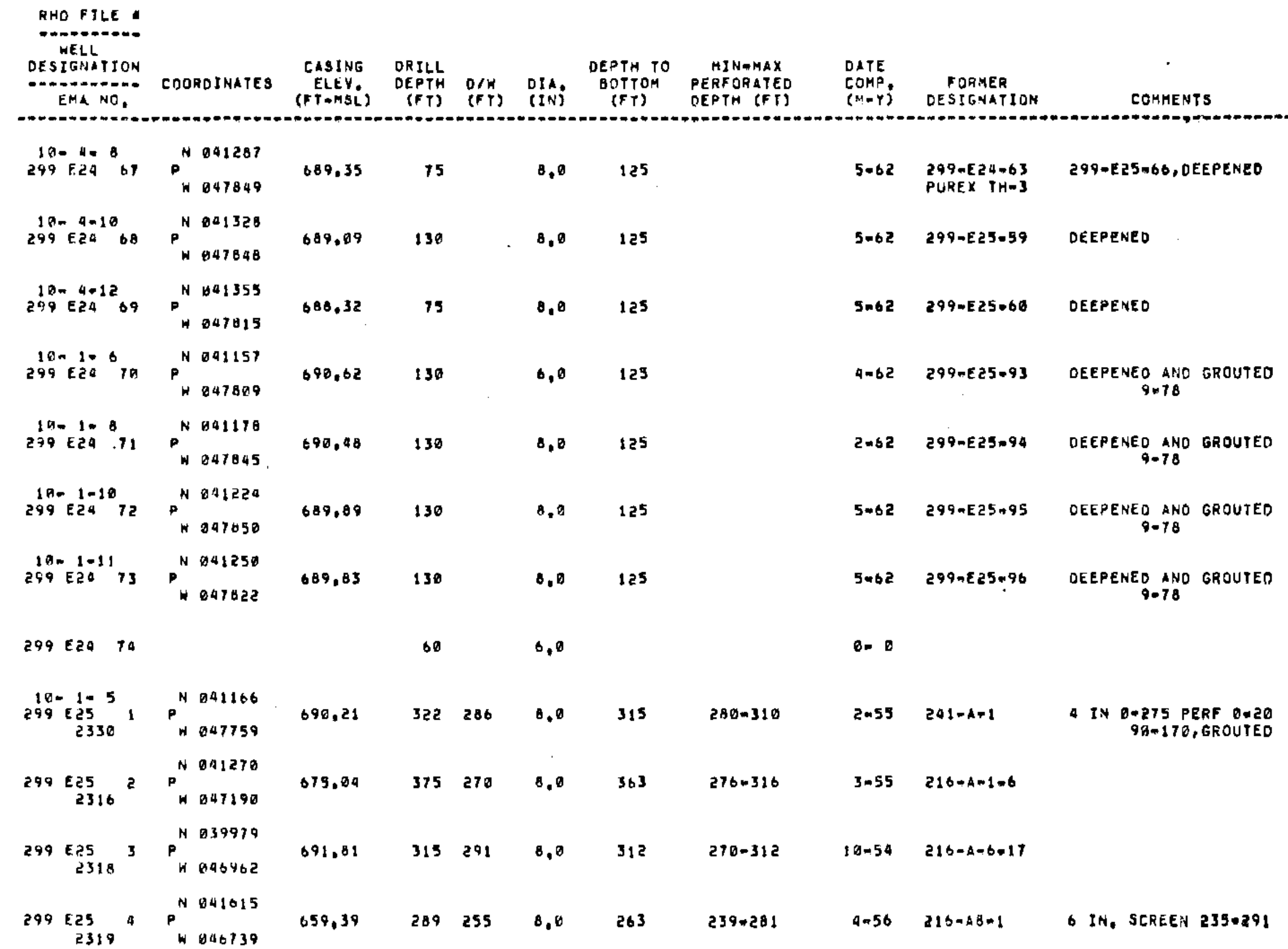




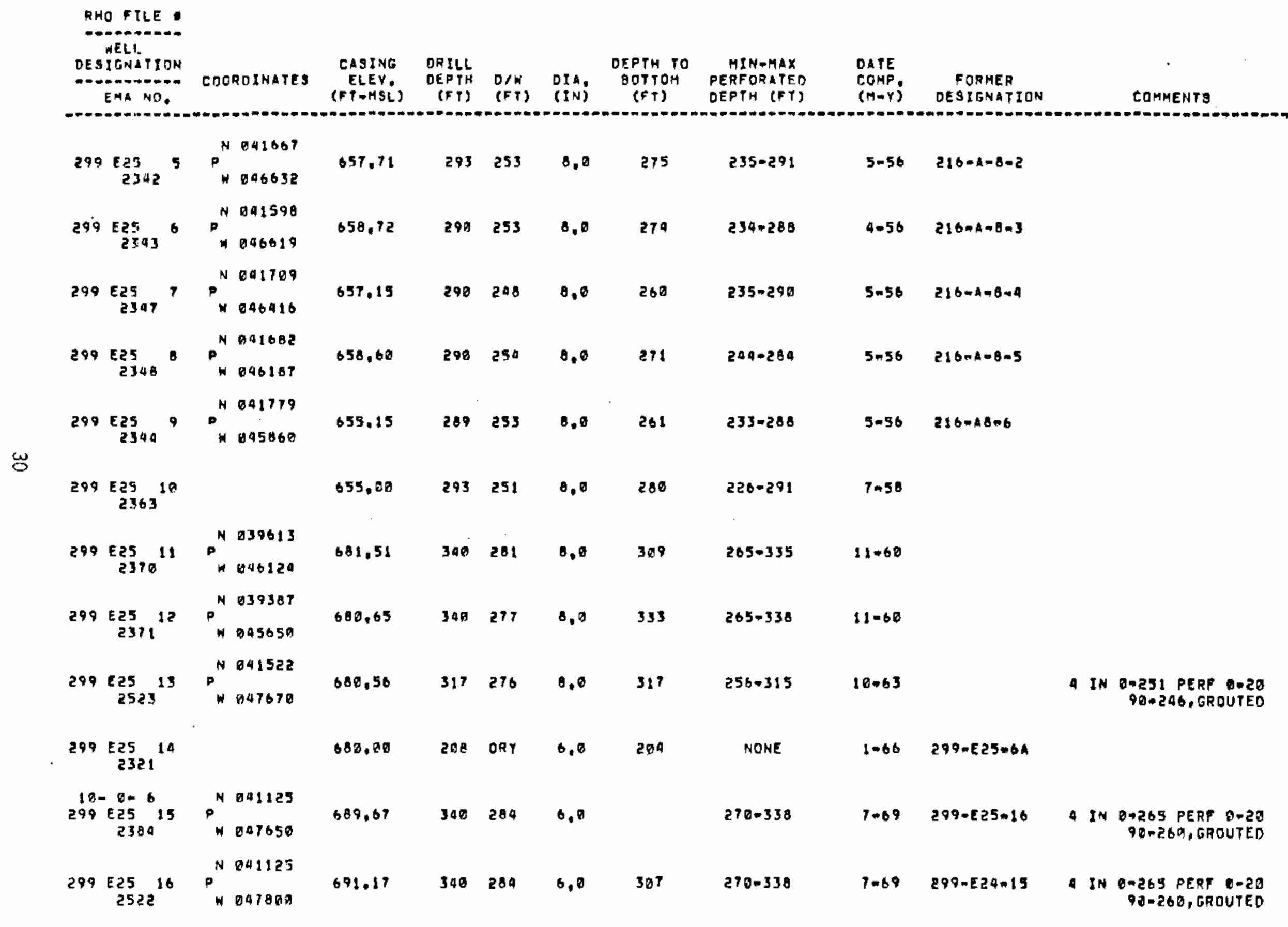




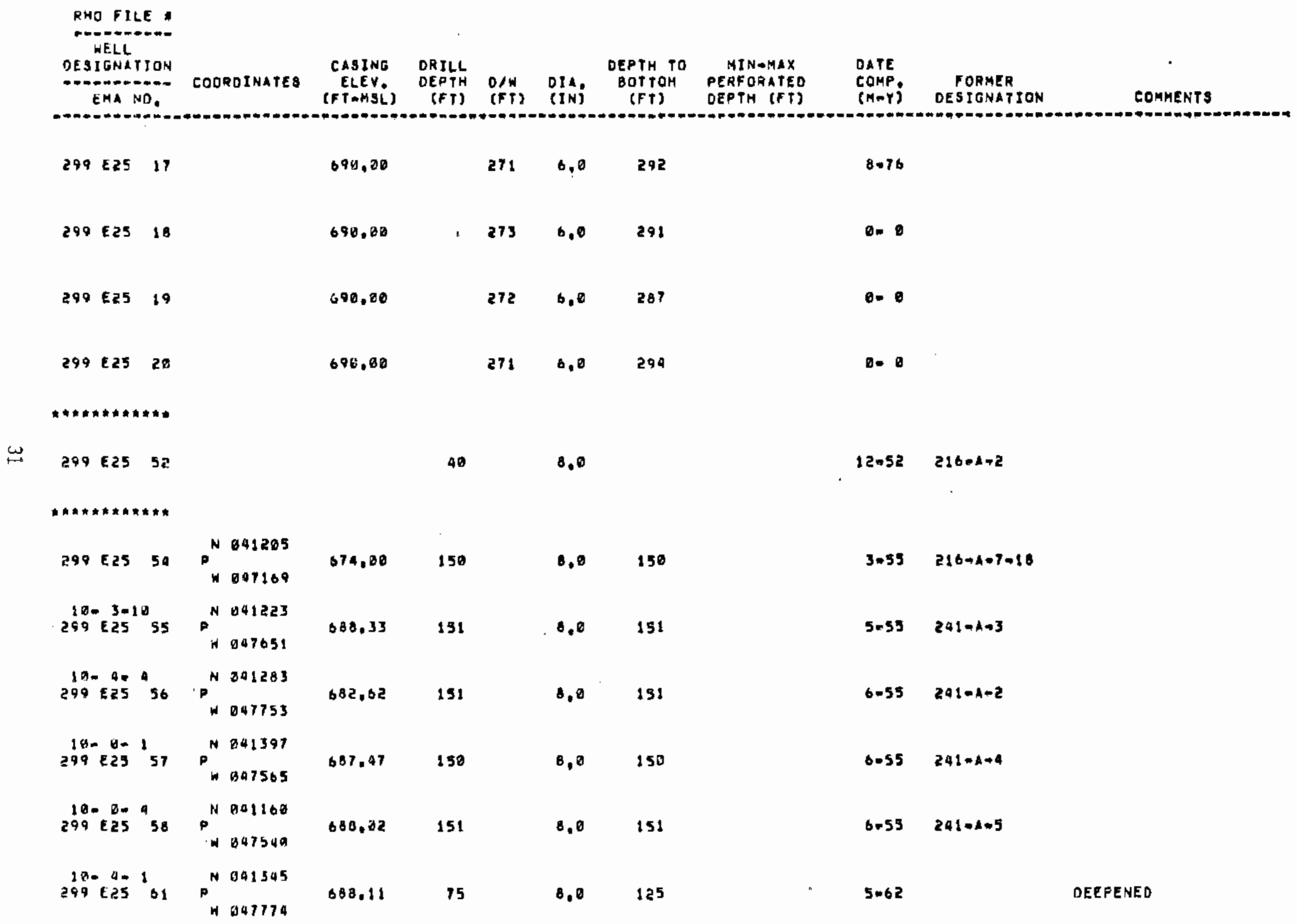




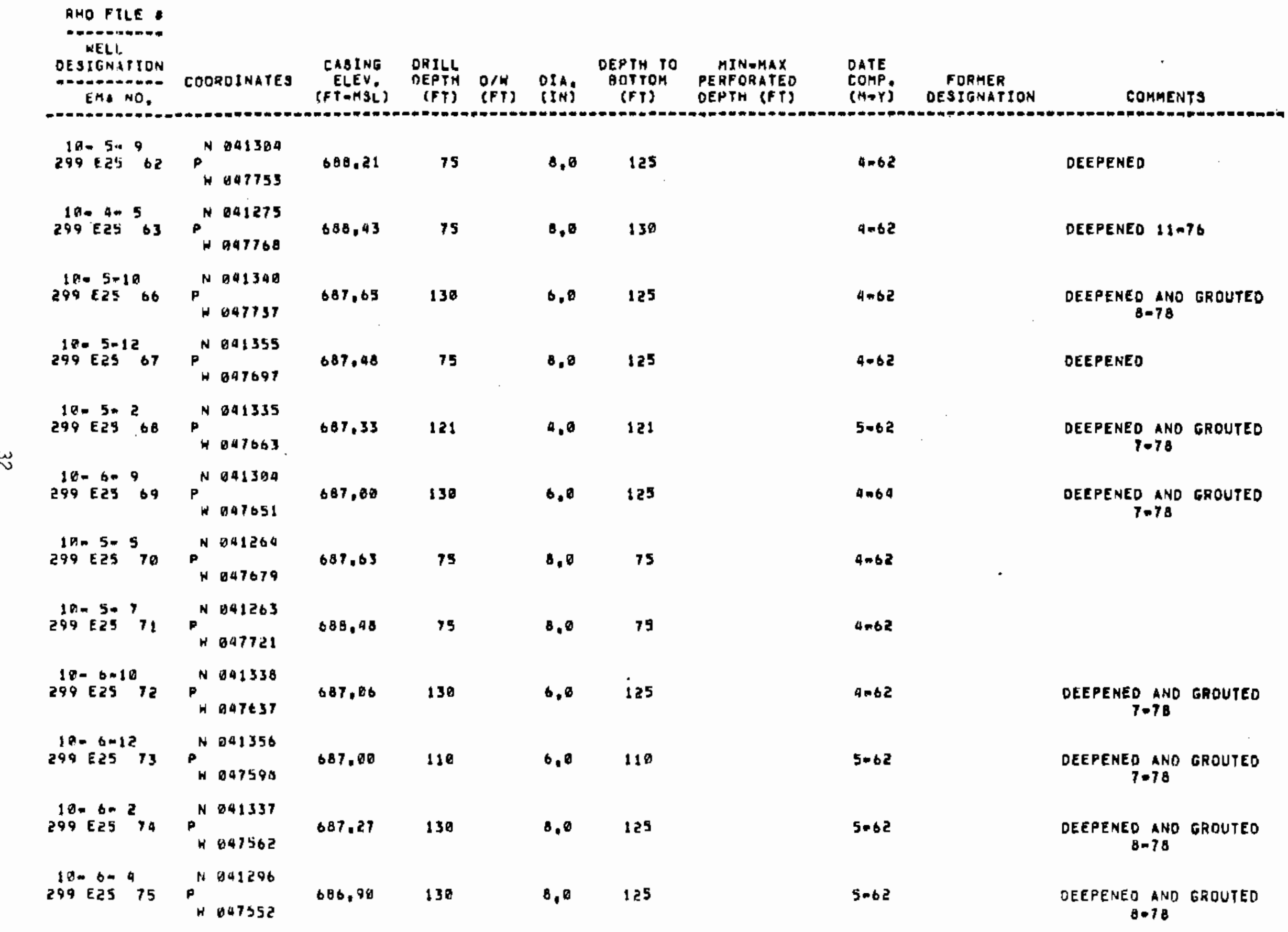




\begin{tabular}{|c|c|c|c|c|c|c|c|c|c|c|c|}
\hline DESIGHATION & COORDINATES & $\begin{array}{l}\text { CASING } \\
\text { ELEV. } \\
\text { (FTAMSL) }\end{array}$ & $\begin{array}{l}\text { DRILL } \\
\text { OEPTH } \\
\text { (FT) }\end{array}$ & $\begin{array}{l}0 / w 1 \\
(F T)\end{array}$ & $\begin{array}{l}\text { DIA: } \\
\text { IIN) }\end{array}$ & $\begin{array}{l}\text { DEPTH TO } \\
\text { BOTTOM } \\
\text { (FT) }\end{array}$ & $\begin{array}{l}\text { MINmAAX } \\
\text { PERFORATED } \\
\text { DEPTH (FT) }\end{array}$ & $\begin{array}{l}\text { DATE } \\
\text { COMP, } \\
\text { (M-Y) }\end{array}$ & $\begin{array}{l}\text { PORMER } \\
\text { DESIGNATION }\end{array}$ & COMMENT & \\
\hline $\begin{array}{l}19-60^{5} \\
29925\end{array}$ & $\begin{array}{l}P_{H}^{N} 041264 \\
H 07577\end{array}$ & 687,15 & 75 & & 8.0 & 75 & & $4=62$ & & & \\
\hline $\begin{array}{c}19-60^{7} \\
299255^{7} 71\end{array}$ & $P_{H}^{N 041264}$ & 687.25 & 130 & & 6.0 & 125 & & $2=62$ & & $\begin{array}{c}\text { DEEPENED AND } \\
7-78\end{array}$ & GROUTED \\
\hline 290 E25 18 & $\begin{array}{l}P^{N} 041250 \\
N 047583\end{array}$ & 687,54 & 130 & & 0.0 & 125 & & $5-62$ & & $\begin{array}{c}\text { DEEPENED AND } \\
9.18\end{array}$ & GROUTED \\
\hline $\begin{array}{l}10-3 \cdot 2 \\
299 \text { E25 }\end{array}$ & $\begin{array}{l}P^{N} 041234 \\
H 47553\end{array}$ & 687,30 & 130 & & 6,0 & 123 & & $9-62$ & & $\begin{array}{c}\text { DEEPENED AND } \\
4-78\end{array}$ & GROUTED \\
\hline $299^{18+35^{4}} 8 a$ & $\begin{array}{l}P^{N} 041180 \\
\end{array}$ & 687,54 & 130 & & 6,0 & 125 & & 4.62 & & $\begin{array}{c}\text { DEEPENED ANO } \\
5.78\end{array}$ & GROUTED \\
\hline $\begin{array}{l}180-3-5 \\
299^{5} E 25^{5} 81\end{array}$ & $\begin{array}{l}\text { N Paiss } \\
\text { W047591 }\end{array}$ & 608,12 & 130 & & 6.0 & 125 & & 4.62 & & $\begin{array}{c}\text { DEEPENEO ANO } \\
g_{n} 78\end{array}$ & GROUTED \\
\hline $\begin{array}{l}19-3-7 \\
299^{25}\end{array}$ & $\begin{array}{l}P_{W}^{N} 041168 \\
\\
N 47652\end{array}$ & 688,32 & 130 & & 6.0 & 125 & & 9062 & & $\begin{array}{c}\text { OEEPENED AND } \\
5=78\end{array}$ & GROUTEO \\
\hline $\begin{array}{l}100203 \\
299 \text { E25 } 83\end{array}$ & $\begin{array}{l}P^{N} 041201 \\
H 047652\end{array}$ & 687,92 & 130 & & 6.0 & 125 & & $9-62$ & & $\begin{array}{c}\text { DEEPENED AND } \\
\frac{5-78}{18}\end{array}$ & GROUTED \\
\hline $299^{16-3=11}$ \& & $\begin{array}{l}P_{W 047655}^{N} 041250 \\
{ }_{N} 040\end{array}$ & 687,53 & 85 & & 0,0 & 75 & & $4=64$ & & & \\
\hline $\begin{array}{l}18 \mathrm{~m} 2=5 \\
299 \text { E25 }\end{array}$ & $P_{N 047670}^{N 041166}$ & 688,57 & 130 & & 6.0 & 125 & & 4.62 & & $\begin{array}{l}\text { DEEPENED AND } \\
6+18\end{array}$ & GROUTED \\
\hline $299^{10-25}{ }^{6} 25$ & $\begin{array}{l}\text { P } \\
\text { W } 8477158\end{array}$ & 689,42 & 90 & & 8,0 & 85 & & $9-62$ & & $\begin{array}{c}\text { DEEPENE O AND } \\
6.78\end{array}$ & GROUTED \\
\hline $\begin{array}{l}19-2 n{ }^{\circ} \\
299^{2}=255^{87}\end{array}$ & $P^{N 041184}$ & 689,42 & 130 & & 6,6 & 125 & & $a \neq b 2$ & & $\begin{array}{l}\text { DEEPENED AND } \\
7.78\end{array}$ & GROUTED \\
\hline
\end{tabular}


RHO FILE

\begin{tabular}{|c|c|c|c|c|c|c|c|c|c|c|c|}
\hline $\begin{array}{l}\text { WELL } \\
\text { OESIGNATION } \\
\text { EMA NO. }\end{array}$ & COOA & QOINATES & $\begin{array}{l}\text { CASING } \\
\text { ELEV. } \\
\text { (FT-HSL) }\end{array}$ & $\begin{array}{l}\text { DRILL } \\
\text { DEFTH } \\
\text { (FT) }\end{array}$ & $\begin{array}{l}D / H \\
(F T)\end{array}$ & DIA: & $\begin{array}{l}\text { DEPTH TO } \\
\text { BOTTOM } \\
\text { (FT) }\end{array}$ & $\begin{array}{l}\text { HINGMAX } \\
\text { PERFORATED } \\
\text { DEPTH (FT) }\end{array}$ & $\begin{array}{l}\text { OATE } \\
\text { COMP. } \\
(H A Y)\end{array}$ & $\begin{array}{l}\text { FORMER } \\
\text { OESIGNATION }\end{array}$ & COMHENT 3 \\
\hline $\begin{array}{l}10-2010 \\
290 \mathrm{E} 2508\end{array}$ & $P^{N}$ & $\begin{array}{l}041226 \\
047746\end{array}$ & 688,68 & 130 & & 6,0 & 125 & & $2 \cdot 62$ & & $\begin{array}{l}\text { DEEPENED AND GROUTED } \\
7.78\end{array}$ \\
\hline $299 \operatorname{ELS}^{2-11} 89$ & $P^{N}$ & $\begin{array}{l}041250 \\
047120\end{array}$ & 686.90 & $13 k$ & & 6,0 & 129 & & $4 \oplus 62$ & & $\begin{array}{l}\text { OEEPENED AND GROUTED } \\
7.78\end{array}$ \\
\hline $\begin{array}{l}10-241 \\
290^{\circ} \text { E25 } 90\end{array}$ & $p^{N}$ & $\begin{array}{l}041240 \\
047670\end{array}$ & 087.87 & 130 & & 6,0 & 125 & & $4=62$ & & $\begin{array}{c}\text { DEEPENED AND GROUTED } \\
7.78\end{array}$ \\
\hline $278-19^{3} \ln ^{3} 91$ & $p_{W}^{N}$ & $\begin{array}{l}041213 \\
041757\end{array}$ & 689.18 & 75 & & 8,0 & 75 & & 4.62 & & \\
\hline $\begin{array}{l}19-10^{\circ} \\
299^{\circ}=2502\end{array}$ & $P^{N}$ & $\begin{array}{l}841172 \\
047770\end{array}$ & 689.57 & 130 & & 8,8 & 125 & & 4.62 & & $\begin{array}{c}\text { DEEPENED AND GROUTED } \\
9-18\end{array}$ \\
\hline $\begin{array}{l}19-101 \\
299 \times 25\end{array}$ & $P^{N}$ & $\begin{array}{l}091247 \\
047701\end{array}$ & 689.09 & 130 & & 0,0 & 129 & & $2-62$ & . & $\begin{array}{c}\text { DEEPENED ANO GROUTED } \\
\qquad .18\end{array}$ \\
\hline $\begin{array}{l}10-5=8 \\
290-258\end{array}$ & $P^{N}$ & $\begin{array}{l}041274 \\
047749\end{array}$ & 688,64 & 56 & & 6.0 & & & 1.66 & & \\
\hline $299<25109$ & $P^{N}$ & $\begin{array}{l}041776 \\
047453\end{array}$ & & 100 & & 6.0 & & & 11.74 & . & \\
\hline $299^{110}=25109$ & $P_{W}^{N}$ & $\begin{array}{l}041753 \\
047429\end{array}$ & & 100 & & 6.0 & & & $12-74$ & & \\
\hline $\begin{array}{lll}11 * 1 * & 4 \\
298 & 25 & 181\end{array}$ & $P^{H}$ & $\begin{array}{l}941710 \\
047429\end{array}$ & & 100 & & 6.0 & & & $t=75$ & & \\
\hline $\begin{array}{ll}11 * 10 & 5 \\
299 & 25 \\
102\end{array}$ & $P^{N}$ & $\begin{array}{l}041082 \\
047458\end{array}$ & & 100 & & 0.0 & & & 1075 & & \\
\hline $\begin{array}{l}110107 \\
299 E 29183\end{array}$ & $P_{W}^{N}$ & $\begin{array}{l}041692 \\
0475190\end{array}$ & & 100 & & 6,0 & & & $t=79$ & & \\
\hline
\end{tabular}




\begin{tabular}{|c|c|c|c|c|c|c|c|c|c|c|}
\hline OESIGNATION & COORDINATES & $\begin{array}{c}\text { CASIHG } \\
\text { ELEV. } \\
\text { (FT\#ASL) }\end{array}$ & $\begin{array}{l}\text { ORILL } \\
\text { OEPTH } \\
\text { (FT) }\end{array}$ & $\begin{array}{l}0 / W \\
(F Y)\end{array}$ & OIN) & $\begin{array}{l}\text { DEPTM TO } \\
\text { BOTTOM } \\
\text { (FT) }\end{array}$ & $\begin{array}{c}\text { MINOHAX } \\
\text { PERFOHATED } \\
\text { DEPTH (FT) }\end{array}$ & $\begin{array}{l}\text { DATE } \\
\text { COMP, } \\
\text { (MAY) }\end{array}$ & $\begin{array}{l}\text { FORMER } \\
\text { OESIGNATION }\end{array}$ & COMMENTS \\
\hline 299 E25 104 & $\begin{array}{l}P^{N} 041726 \\
N 047520\end{array}$ & 681.64 & 180 & & 6,0 & & & 12.74 & & \\
\hline $\begin{array}{c}11-1=11 \\
299 \varepsilon 25105\end{array}$ & $\begin{array}{l}P^{N} 041773 \\
W 027495\end{array}$ & & 100 & & 6,0 & & & $12=74$ & & \\
\hline $\begin{array}{rl}11-25 & 2 \\
29925106 & \end{array}$ & $\begin{array}{l}\text { N } 941648 \\
W 047429\end{array}$ & & 100 & & 6,0 & & & 1.75 & & \\
\hline $\begin{array}{lll}11-020 & 9 \\
290 & 25 & 107\end{array}$ & $\begin{array}{l}P_{W}^{N} 041600 \\
W 47428\end{array}$ & & 100 & & 6.0 & & & $2 \pi 75$ & & \\
\hline $\begin{array}{rll}11 \% & 2 \pi & 5 \\
299 & 25 & 100\end{array}$ & $\begin{array}{l}N 841585 \\
W 847455 .\end{array}$ & . & 100 & & 6,0 & & & $2+73$ & & \\
\hline $299 \varepsilon 25109$ & $\begin{array}{l}P^{N} 801985 \\
+807495\end{array}$ & & 100 & & 6.8 & & & $2-75$ & & \\
\hline $\begin{array}{lll}11-2 * & 0 \\
289 & 25 & 110\end{array}$ & $\begin{array}{l}P^{N} 041595 \\
N 047520\end{array}$ & & 100 & & 6.0 & & & $2 \times 73$ & . & CAFPEO BELOW GRADE \\
\hline $\begin{array}{l}11+2+18 \\
299 \\
\text { E } 25111\end{array}$ & $\begin{array}{l}P_{n}^{N} 041044 \\
n 047519\end{array}$ & & 100 & & $B, 0$ & & & $2-73$ & & \\
\hline $\begin{array}{c}11-2-11 \\
299 E 25112\end{array}$ & $\begin{array}{l}P^{N} 041069 \\
H \quad 84799\end{array}$ & & 100 & & 6,0 & & & $\{-75$ & & \\
\hline $\begin{array}{lll}11-3 \pi & 2 \\
299 E 25 & 113\end{array}$ & $\begin{array}{l}p^{N 041768} \\
404532\end{array}$ & & 180 & & 6,0 & & & $1=75$ & & \\
\hline 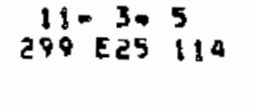 & $\begin{array}{l}P^{N} 001691 \\
N 047540\end{array}$ & 682,62 & 208 & & 6,8 & & & 12.74 & & \\
\hline $\begin{array}{lll}11-3-30 & 1 \\
249 & 29 & 115\end{array}$ & $\begin{array}{l}p^{N} 841691 \\
W 04589\end{array}$ & 683,00 & 100 & & 0,0 & & & $2-75$ & & \\
\hline
\end{tabular}




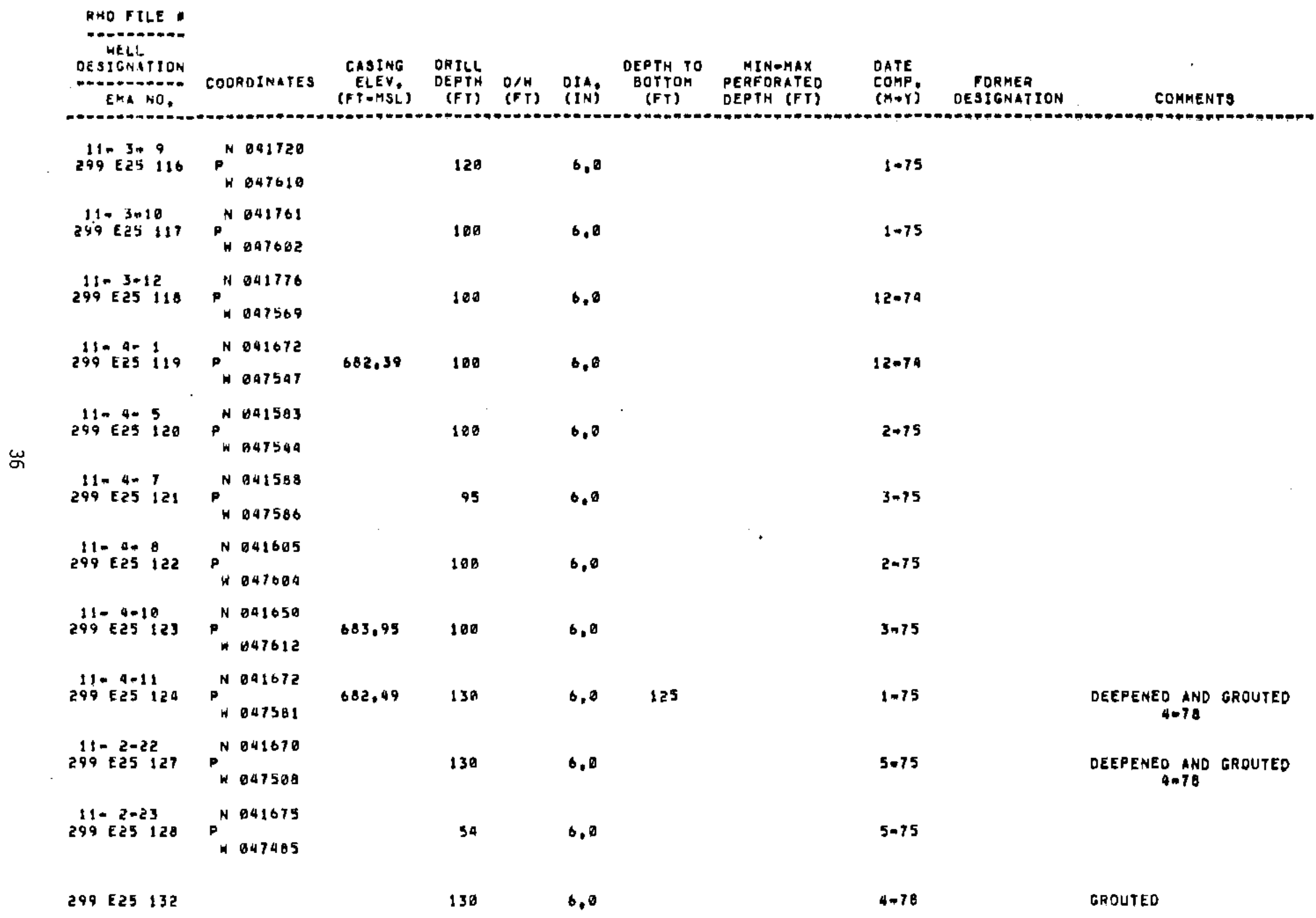




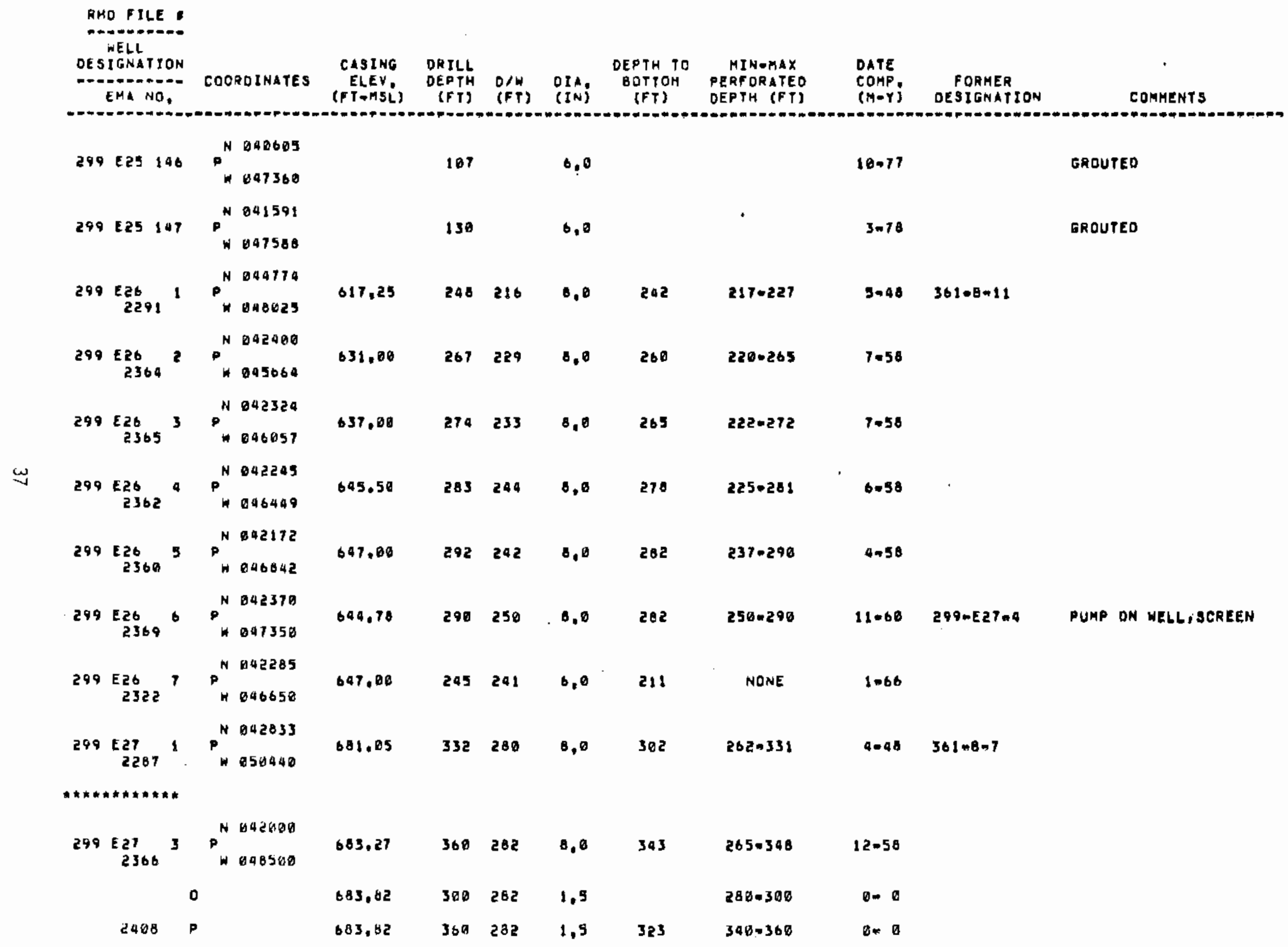


RHO FILE -

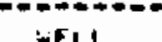

DESIGNATION

COATION

EH NO.

CASING

ELEY OETH

ORILL
OEPTH DIW DIA, BDTH TO MINOHAX DEPTH (FT)

DATE

COMP, FORMER

(MNY) DESIGNATION

COMMENTS

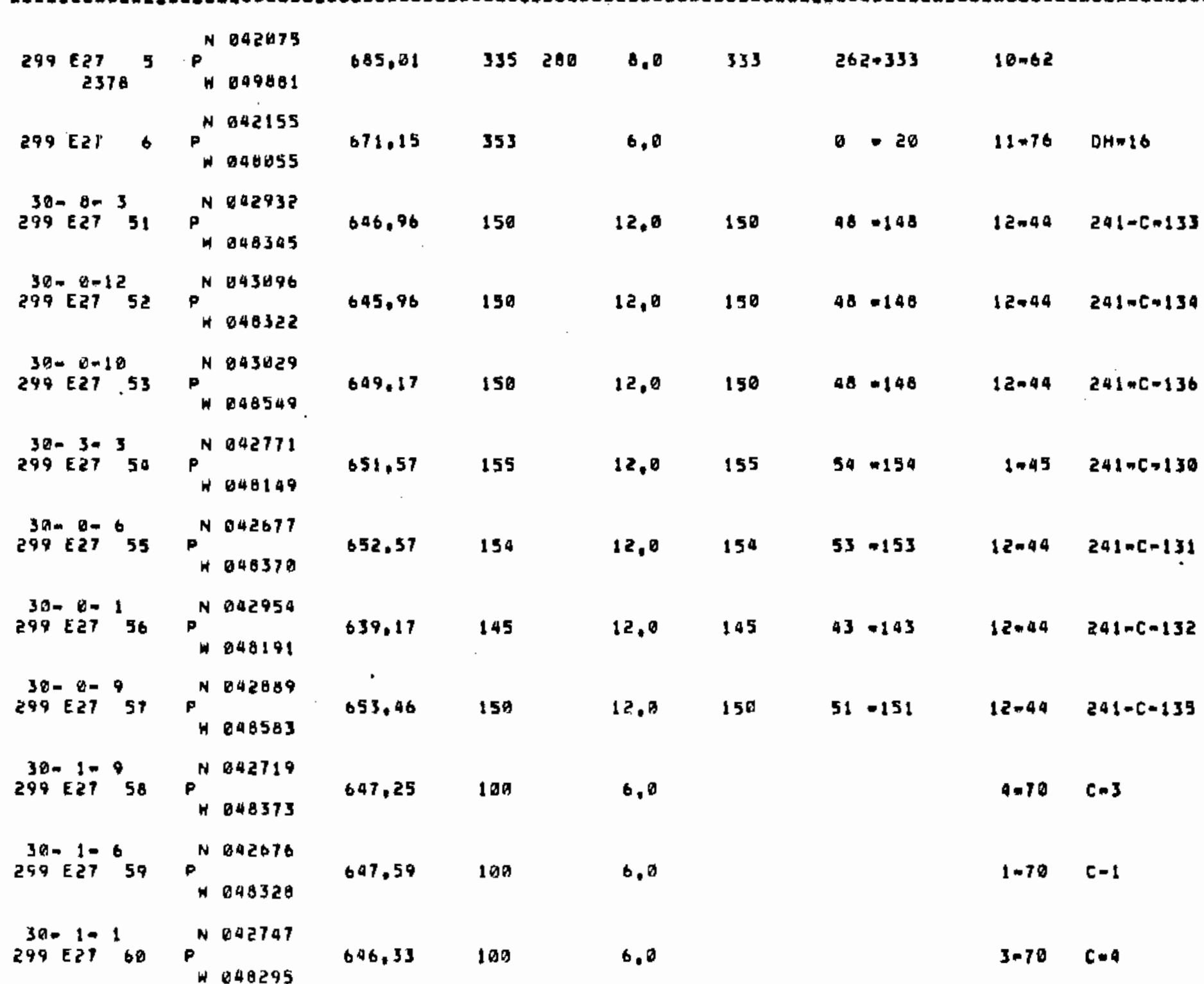




\begin{tabular}{|c|c|c|c|c|c|c|c|c|c|}
\hline $\begin{array}{l}\text { HELL } \\
\text { OESIGNATION } \\
\text { EMA NOE. }\end{array}$ & COOROINATES & $\begin{array}{l}\text { CABING } \\
\text { ELEY, } \\
\text { (FTAMSL) }\end{array}$ & $\begin{array}{l}\text { DRILL } \\
\text { DEPTH } \\
\text { (FT) }\end{array}$ & $\begin{array}{l}0 / W \\
(F T)\end{array}$ & DING & $\begin{array}{l}\text { OEPTH TO } \\
\text { BOTTOM } \\
\text { (FT) }\end{array}$ & $\begin{array}{l}\text { MINVMAX } \\
\text { PEFFORATED } \\
\text { DEPTH (FT) }\end{array}$ & $\begin{array}{l}\text { OATE } \\
\text { CORP, } \\
(M=Y)\end{array}$ & $\begin{array}{l}\text { FORMER } \\
\text { DESIGNATION COMMENTS }\end{array}$ \\
\hline $290 \times 1-127^{12} 61$ & $\begin{array}{l}P^{N} 892762 \\
N 098339\end{array}$ & 646.82 & 100 & & 6.0 & & & $3-70$ & {$[-3$} \\
\hline $\begin{array}{l}30-1159 \\
299 E 27\end{array}$ & $P^{N} 0403002$ & 646,37 & 100 & & 6,0 & & & $3-10$ & $c=1$ \\
\hline $\begin{array}{l}3 A-11-1 \\
290 \times 27 \text { 63 }\end{array}$ & $D_{N 048441}^{N 04036}$ & 605,77 & 100 & & 6,0 & & & $2-70$ & $c-2$ \\
\hline $\begin{array}{l}30-11=6 \\
299 \text { E27 } 64\end{array}$ & $\begin{array}{l}\rho^{N 042957} \\
4098969\end{array}$ & 696.09 & 100 & & 6,0 & & & $2-76$ & $c=3$ \\
\hline $299=27^{12}$ BS & $\begin{array}{l}P^{N 042835} \\
\text { No48045s }\end{array}$ & 647,21 & 135 & & 6,0 & & & $12=72$ & \\
\hline $\begin{array}{l}30-4=8 \\
299 \text { E2T } 66\end{array}$ & $\begin{array}{l}P^{N 042750} \\
N 048437\end{array}$ & 649,06 & 145 & & 6.0 & & & $11-72$ & \\
\hline $\begin{array}{l}38-4-2 \\
299 \text { E27 }\end{array}$ & $P^{N} 042827$ & 646,65 & 130 & & 6,8 & & & $12 \cdot 72$ & \\
\hline $\begin{array}{l}38-5=16 \\
289 E 2768\end{array}$ & $\begin{array}{l}P_{N}^{N 042893} \\
N 040306\end{array}$ & 646.23 & 133 & & 6.0 & & & $11-72$ & \\
\hline $\begin{array}{l}30-5-4 \\
290 \mathrm{E2} \quad 69\end{array}$ & $P_{H}^{N 042825}$ & 646.07 & 120 & & 6.0 & & & $12-12$ & \\
\hline $\begin{array}{l}30-5=2 \\
290-527^{2} 70\end{array}$ & $P^{N 042893}$ & 643.70 & 130 & & 6.8 & & & $11-72$ & \\
\hline 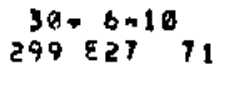 & $\begin{array}{l}P_{N}^{N} \times 42463 \\
N 48291\end{array}$ & 645,31 & 130 & & 6,0 & & & $11-72$ & \\
\hline $289 \mathrm{E} 27^{30} 72$ & $P_{N}^{N 042967}$ & 645,33 & 125 & & 6,0 & - & & $11=72$ & \\
\hline
\end{tabular}


RHO FILE

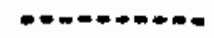

HELL.

OESIGNTIIION

EODROINATES

EMA NO,

CASING ORILL

OEPTH

(FT-MSi)

OEPTH
(FT)

(FT)

OIA. BEPTHTO

MINEMAX
PERFORATEO PEAF ORATED
DEPTH (FT)

DATE

30. b- a N 045897

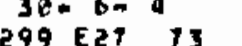

N

$64.71 \quad 130$

$b, 8$

$11=72$

3B-3\% 1 N842981

290 E2T $7 A$ P

an

$30 m 503042861$

$299 E 27 \quad 75 \quad P \quad 098140$

4098140

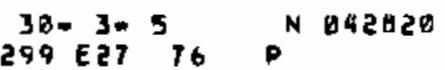

* 048165

30.3.7 N 742825

$299 E 2777$

H 040215

$645.64 \quad 109$

$B, 8$

9074

290 E27 $78 P^{N 042861}$

1. 340231

100

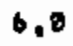

$b-74$

$38-403 \quad N 042790$

$299 E 27 \quad 79 \quad P$

H 108352

$646.60 \quad 160$

6.6

$b=74$

389055 F 2042748

$647.08 \quad 100$

6.0

$7 \sim 74$

$30=503$ N 342861

299 E27 81 P

1 88282

100

$b, 0$

$9-79$

$39-505$ 5 $p^{N 042813}$

646,21

100

$B .8$

$b=7 a$

3日-5.9 N042061

$\begin{array}{lllll}299 & E 27 & 83 & P \\ & & & \\ & & \end{array} 48375$

$600,61 \quad 109$

6.0

0.74

3a- $b=3$

N 042933

299 E2T $84 \quad P$

$P$

$649.80 \quad 100$

6.0

$6-74$ 


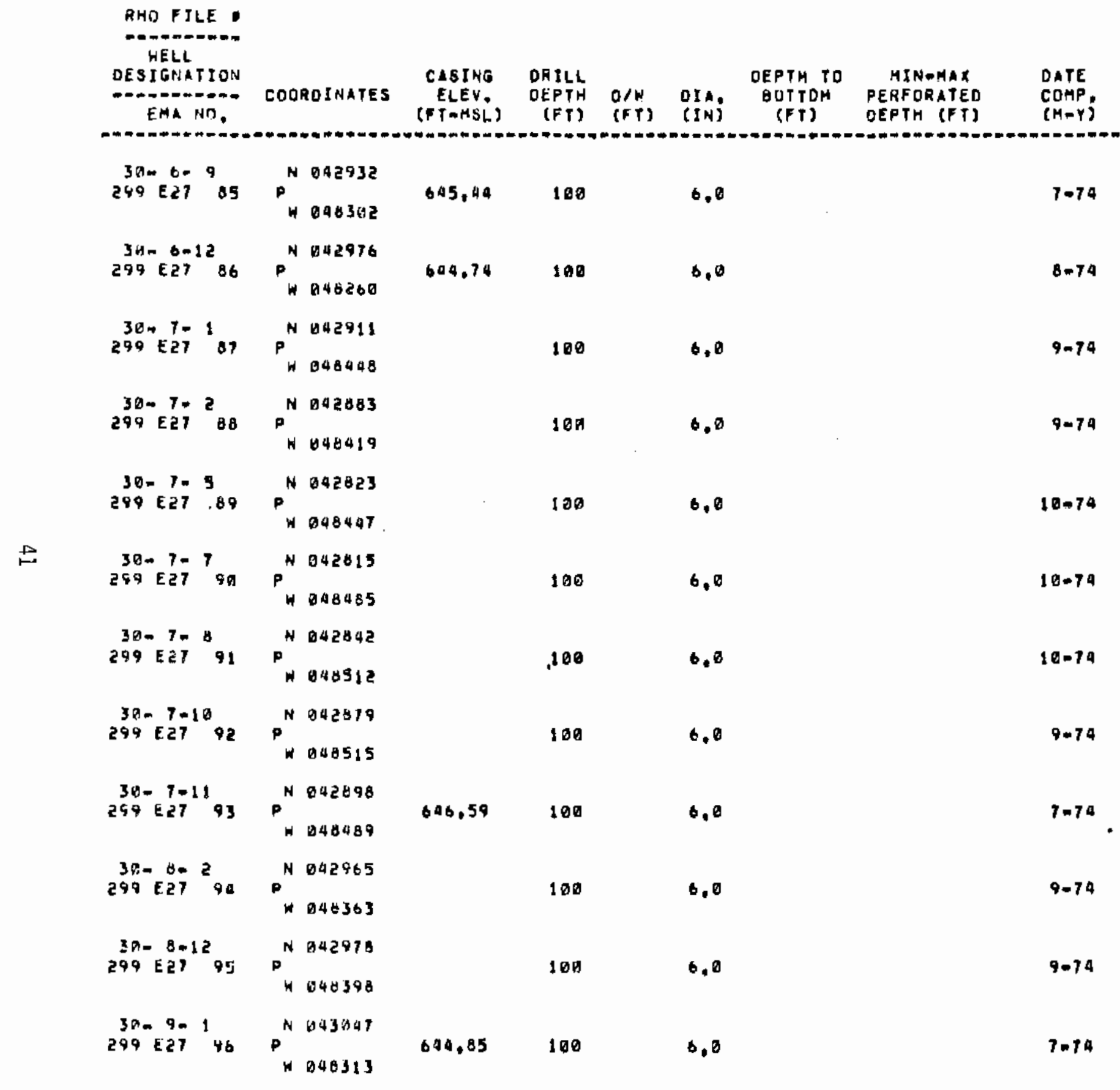




\begin{tabular}{|c|c|c|c|c|c|c|c|c|c|}
\hline OESIGNATION & CODROINATES & $\begin{array}{l}\text { CASING } \\
\text { ELEV. } \\
(F T-M S L)\end{array}$ & $\begin{array}{l}\text { DRILL } \\
\text { DEPTH } \\
\text { (FT) }\end{array}$ & (FT) & $\begin{array}{l}\text { OIA; } \\
\text { (IN) }\end{array}$ & $\begin{array}{l}\text { DEPTH TO } \\
\text { BDTTOM } \\
(F T)\end{array}$ & $\begin{array}{l}\text { MINOMAX } \\
\text { PEAFORATED } \\
\text { DEPYH (FI) }\end{array}$ & $\begin{array}{l}\text { OATE } \\
\text { COMP, } \\
(M-Y)\end{array}$ & $\begin{array}{l}\text { FORHEQ } \\
\text { OESIGNATION COMMENTS }\end{array}$ \\
\hline $\begin{array}{l}30.9-2 \\
299 \text { ह2. }\end{array}$ & $\begin{array}{l}P^{N} 043023 \\
H 048284\end{array}$ & 645.17 & 100 & & 6.0 & & & $6=74$ & \\
\hline $\begin{array}{r}300-97^{6} \\
299 \times 27^{3} 98\end{array}$ & $P_{W 048327}^{N 042956}$ & & 100 & & 6.0 & & & $9=74$ & \\
\hline $\begin{array}{l}30 * 9 \times 10 \\
299 E 27^{99} 99\end{array}$ & $P_{H}^{N 043026}$ & 645.43 & 100 & & 6.6 & & & 7.74 & \\
\hline $\begin{array}{l}30-9-11 \\
299 E 27100\end{array}$ & $P^{N} 0030405$ & 649.99 & 100 & & 6,0 & & & $7 \cdot 74$ & \\
\hline $\begin{array}{l}30-10-7 \\
299 E 27\end{array}$ & $P^{N} 04852989$ & & 180 & & 6.0 & & & $9=74$ & \\
\hline $\begin{array}{c}30-10=2 \\
299<27102\end{array}$ & $P_{N 048094}^{N 042945}$ & 646,52 & 100 & & $B .0$ & & & 9.74 & \\
\hline $\begin{array}{c}30-110 \\
299 \text { E27 } 103\end{array}$ & $\begin{array}{l}P^{N} 042926 \\
N 09585\end{array}$ & & 108 & & 6.8 & & & 9.74 & \\
\hline $\begin{array}{c}38-10 \div-11 \\
299 E 21104\end{array}$ & $P^{N} 042967$ & & 100 & & 6,8 & & & 9.75 & \\
\hline 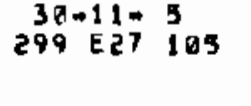 & $P^{N} 842964$ & & 100 & & 6.0 & & & 9.75 & \\
\hline $\begin{array}{c}30-11-11 \\
299 \text { Eट7 } 106\end{array}$ & $P_{N}^{N} 843048435$ & & 100 & & 6.0 & - & & $4=75$ & \\
\hline $\begin{array}{c}30-12 * 1 \\
299 E 27107\end{array}$ & $P^{N} 843120$ & & 100 & & 6.0 & & & $4=75$ & \\
\hline $\begin{array}{rl}38-127 & 3 \\
299 E 27 & 108\end{array}$ & $P^{N} 043488352$ & & 100 & & 6,0 & & & $a=73$ & \\
\hline
\end{tabular}




\begin{tabular}{|c|c|c|c|c|c|c|c|c|c|c|}
\hline 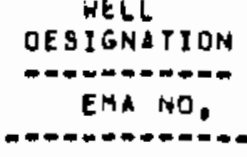 & COORDINATES & $\begin{array}{c}\text { CASING } \\
\text { ELEY, } \\
\text { (FTAMSL) }\end{array}$ & $\begin{array}{l}\text { ORILL } \\
\text { OEPTH } \\
\text { (FT) }\end{array}$ & \begin{tabular}{l}
$\square / N$ \\
$(F T)$ \\
\hdashline
\end{tabular} & DIN & $\begin{array}{c}\text { OEPTH TO } \\
\text { BOTTOH } \\
\text { (FT) }\end{array}$ & $\begin{array}{l}\text { HIN-MAX } \\
\text { PEAFORATEO } \\
\text { DEPTH (FT) }\end{array}$ & $\begin{array}{l}\text { DATE } \\
\text { COMP, } \\
(M=Y)\end{array}$ & $\begin{array}{l}\text { FORMER } \\
\text { OESIGNATION }\end{array}$ & COMMENTS \\
\hline $\begin{array}{r}37-12=9 \\
299 E 27199\end{array}$ & $\begin{array}{l}P_{N 043074}^{N} \\
N 048440\end{array}$ & & 100 & & 6.0 & & & $4=75$ & & \\
\hline $\begin{array}{l}30-40 \\
299 \text { E2t l15 }\end{array}$ & $\begin{array}{l}P^{N} 842829 \\
N 04378\end{array}$ & & 50 & & 6,0 & & & $7=74$ & & \\
\hline $290 \times 277^{30} 116$ & $\begin{array}{l}P_{N 042867}^{N} 040365 \\
{ }^{N}\end{array}$ & & 50 & & 8,0 & & & $7=74$ & & \\
\hline $\begin{array}{rll}39+50 & 0 \\
299 & 27 & 117\end{array}$ & $\begin{array}{l}P^{N} 042838 \\
W 048367\end{array}$ & & 50 & & 6.0 & & & $7=74$ & & \\
\hline $\begin{array}{rl}30-5-7 \\
299-527 & 118\end{array}$ & $\begin{array}{l}P^{N} 042826 \\
H 048353\end{array}$ & & 70 & & 6,8 & & & 7.74 & & \\
\hline $\begin{array}{l}3005=0 \\
249 \text { E27 } 119\end{array}$ & $0^{N} 042814$ & & 60 & & 6,6 & & & $7+74$ & . & \\
\hline $299 E 27 \quad 120$ & & & 60 & & 0,0 & & & $3-77$ & & GROUTED \\
\hline 299 ERT 121 & & & 66 & & 6.0 & & & $3-77$ & & GROUTED \\
\hline 299 E27 122 & & & 60 & & 6,8 & & & $3-77$ & & GROUTEO \\
\hline $299527 \quad 123$ & & & 60 & & 6,0 & & & $0-0$ & & \\
\hline $299 E 27124$ & & & 60 & & 6,0 & & & $3-77$ & & GROUTED \\
\hline $\begin{array}{c}38-12-25 \\
299 E 27125\end{array}$ & $\begin{array}{l}P^{N} 043116 \\
N 048387\end{array}$ & & 130 & & 6,6 & & & $4-78$ & & GROUTED \\
\hline
\end{tabular}




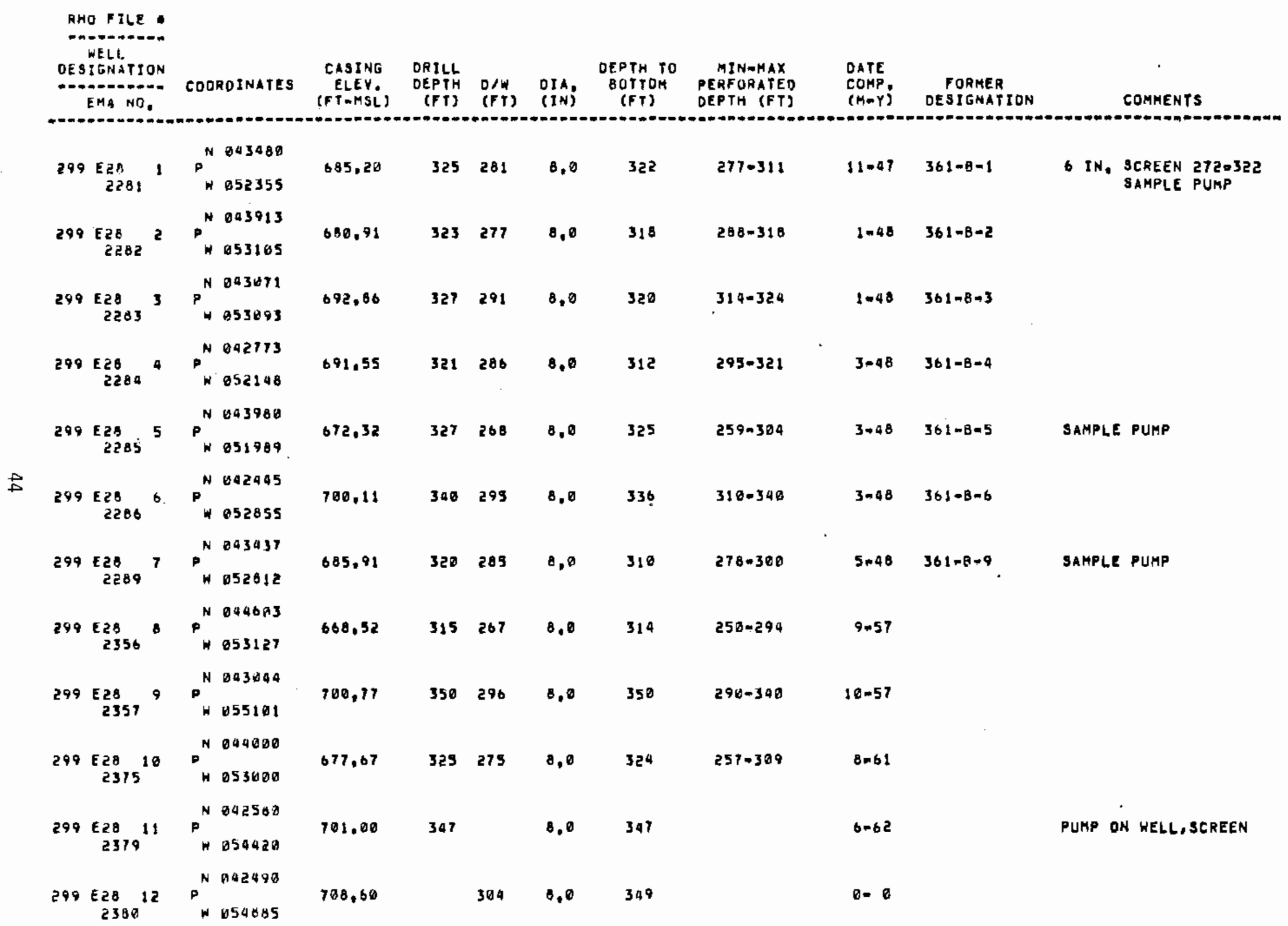




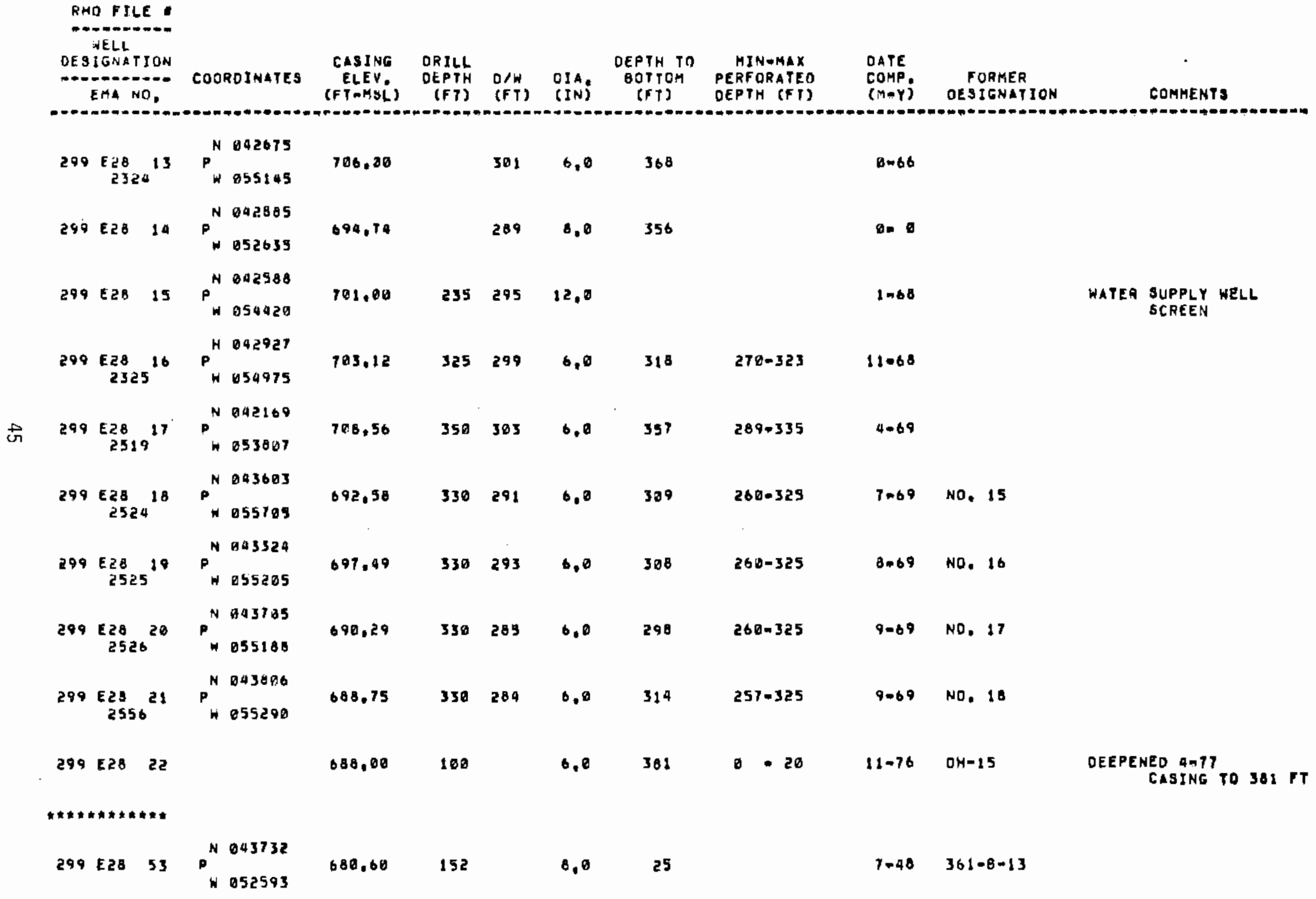


RHO FILE

$$
\text { HELL }
$$

OESIGNATION

EMA NO.

COQROINATES

CASING

ELEV DEPTH

OEPTH TO

DEPTH (FT)

DATE

COMP: FORMEA

$M=Y$

DES I GNAT ION

COMMENTS

N
N43735

299 E2A 5a P $P^{N} 053732543$

680.43

$8,0 \quad 156$

$7-48 \quad 361-8-14$

$299 E 2855 p^{N 043702}$

$680,30 \quad 150$

0,0150

$7=48 \quad 361=B-15$

$299 E 2856 P^{N} 043862$

$076.25 \quad 150$

$8,0 \quad 150$

$7=48 \quad 361-8-16$

$209 E 255 P^{N 043662}$

678,24

0.0150

$7-40 \quad 361-0.17$

299 E20 50 N 043916

$675,78 \quad 150$

8,0145

$7-48 \quad 361 \times 9+18$

B

290 E25 50 OA5916

676.45

$8.6 \quad 141$

$7-48 \quad 361-8-19$

2N045977

299 E28 OD P
W052618
N 843782

259 E2B b1 ${ }^{N} 843782$

679.65

$8,0 \quad 145$

$7-98361-8-29$

$680.51-150$

0.0150

8.48 361-8-12

$299 E 28$ o2 $P^{N 093837}$

678.20

0,09

$10-48 \quad 361-0-548$

$299 E 2063 P^{N 043009}$

$700.00 \quad 50$

8.050

$9-50 \quad c-362-1$

$299 E 26 \quad 64 P^{N} 043000$

$100,00 \quad 48$

$6,0 \quad 33$

2-51 216-ER-1

$299 k 2665 p^{N 043060}$

$720.00 \quad 48$

0.080

2-51 216mEH-2

DEEPENEO 


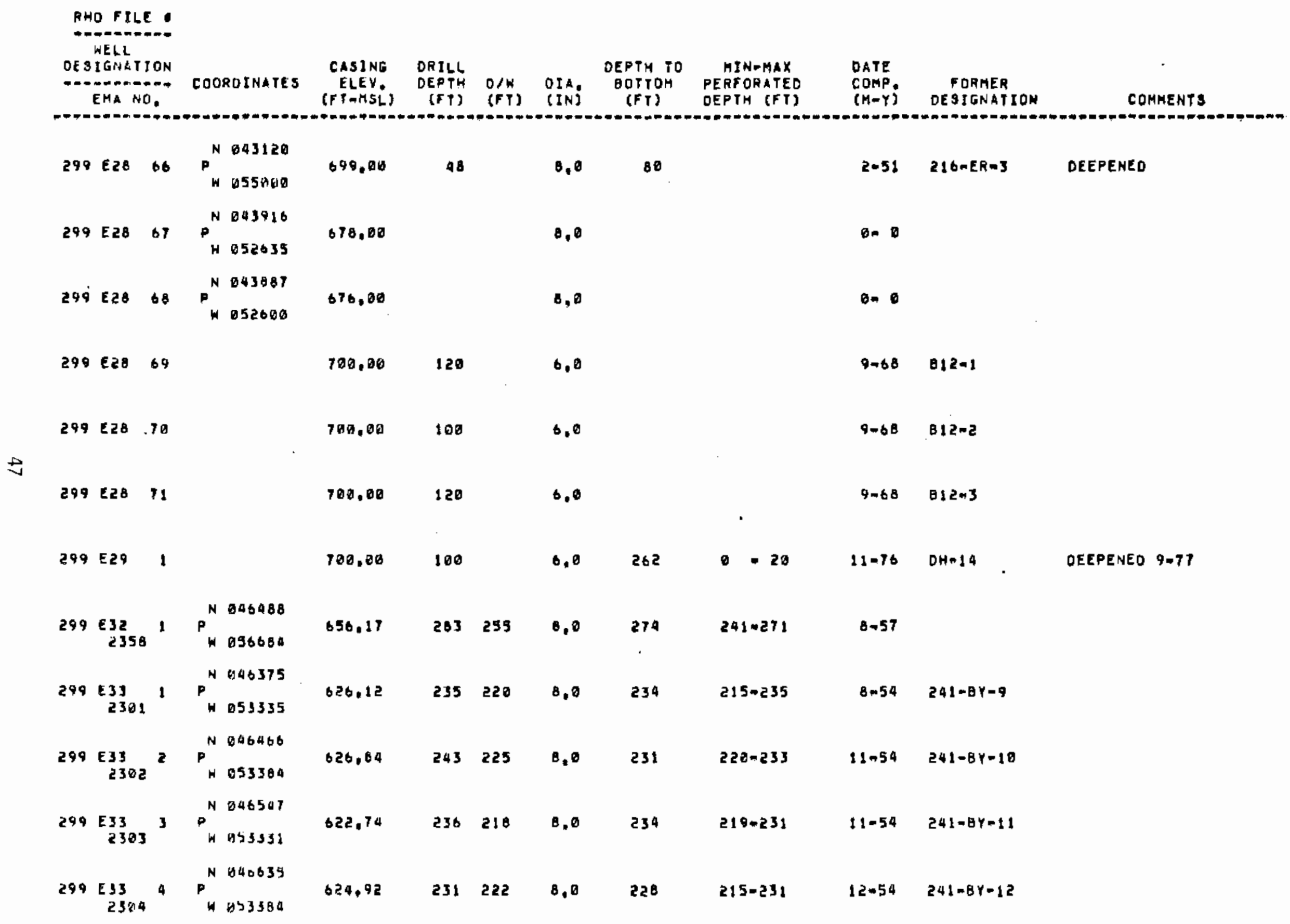




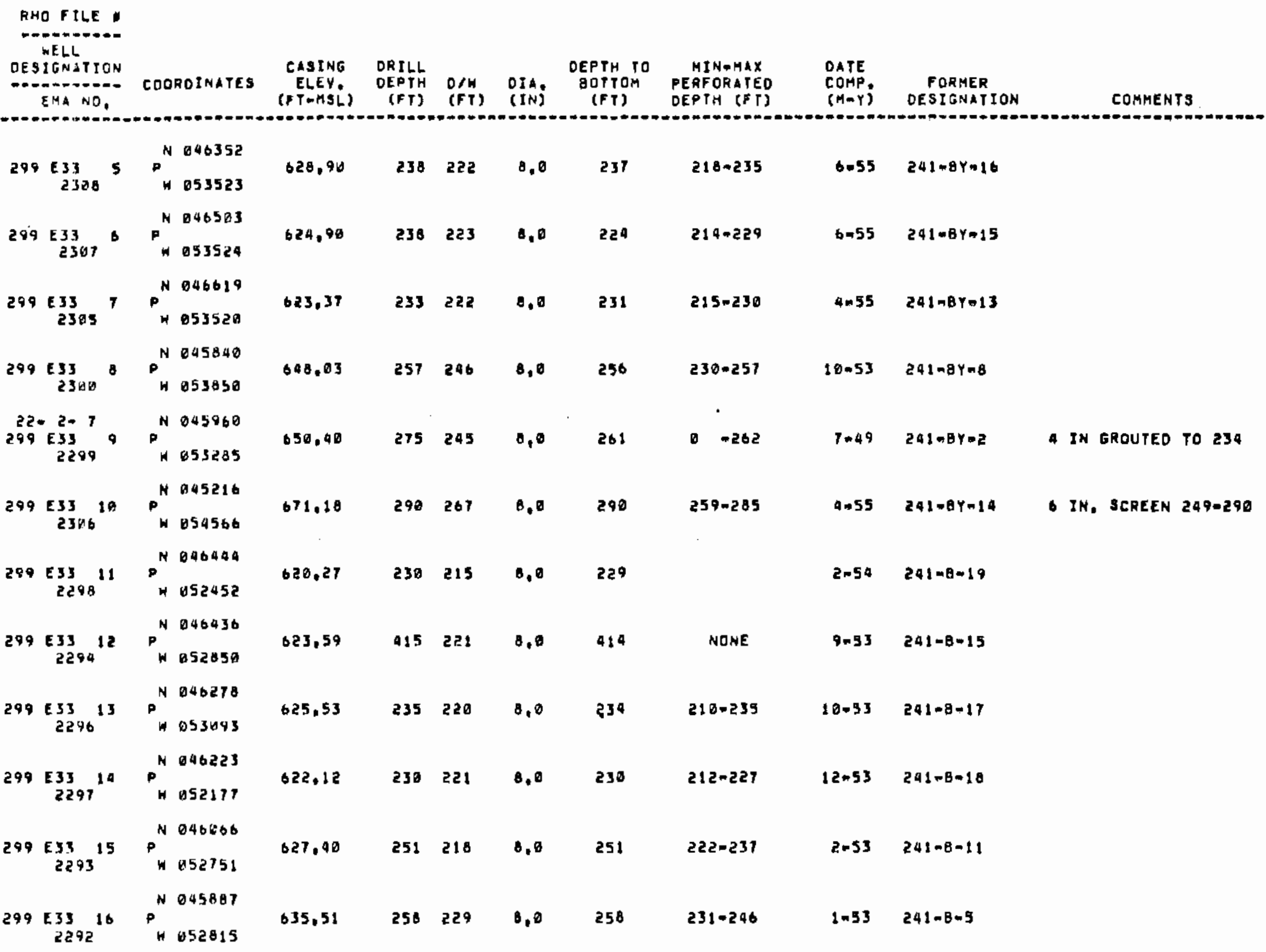




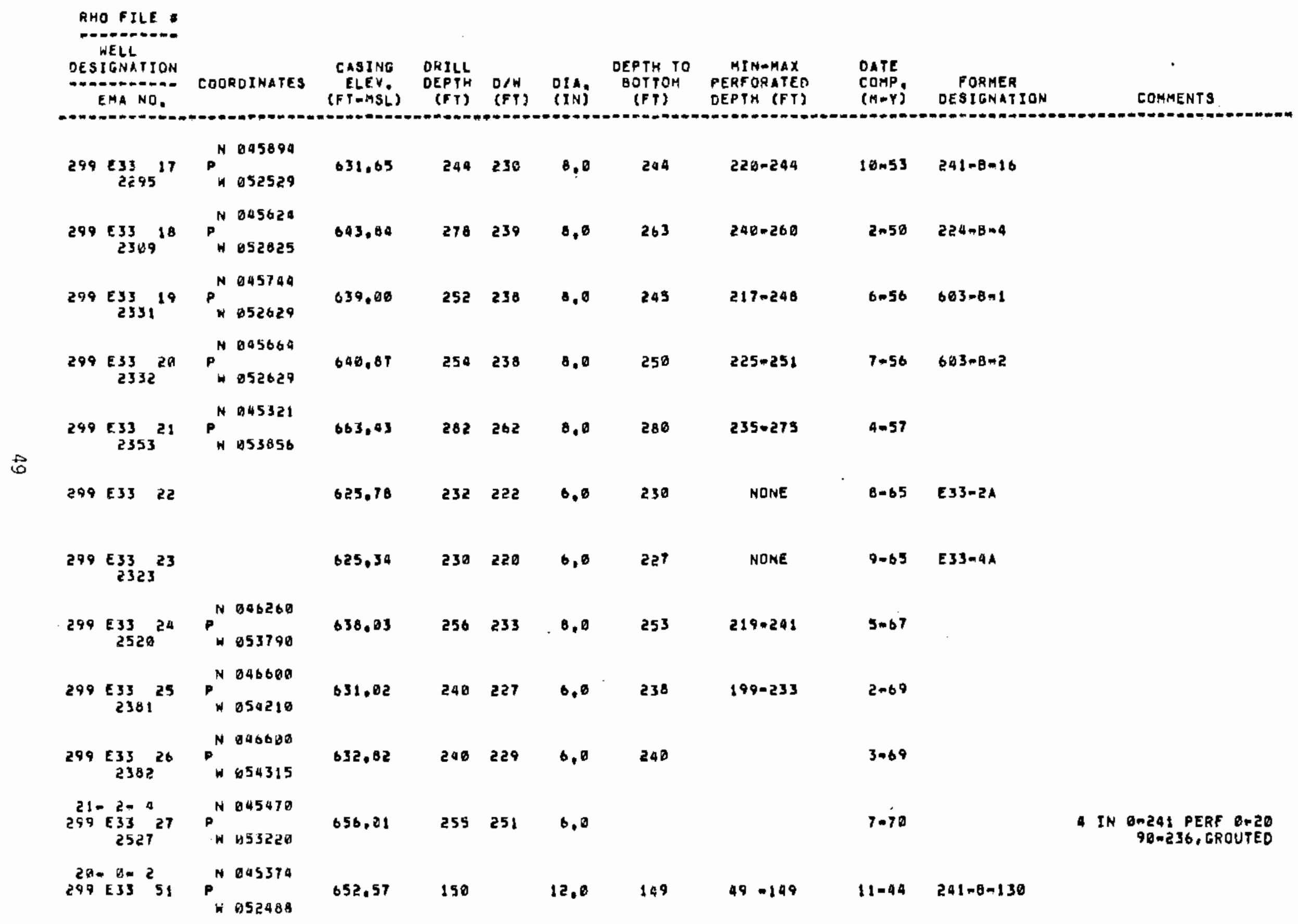


RHO FILE

$$
\text { HELL }
$$

DESIGNATION

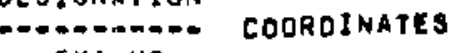

EM NO.

CASING ORILL

OEPTH TO MINAMAX

$\begin{array}{lllll}\text { (FTANSL) (FT) (FT) (IN) BOTTOM PERFORATED } & \text { (FT) OEPTH (FT) }\end{array}$

DATE

COMP, FORMER

(H-Y) DESIGNATION

COAHENTS

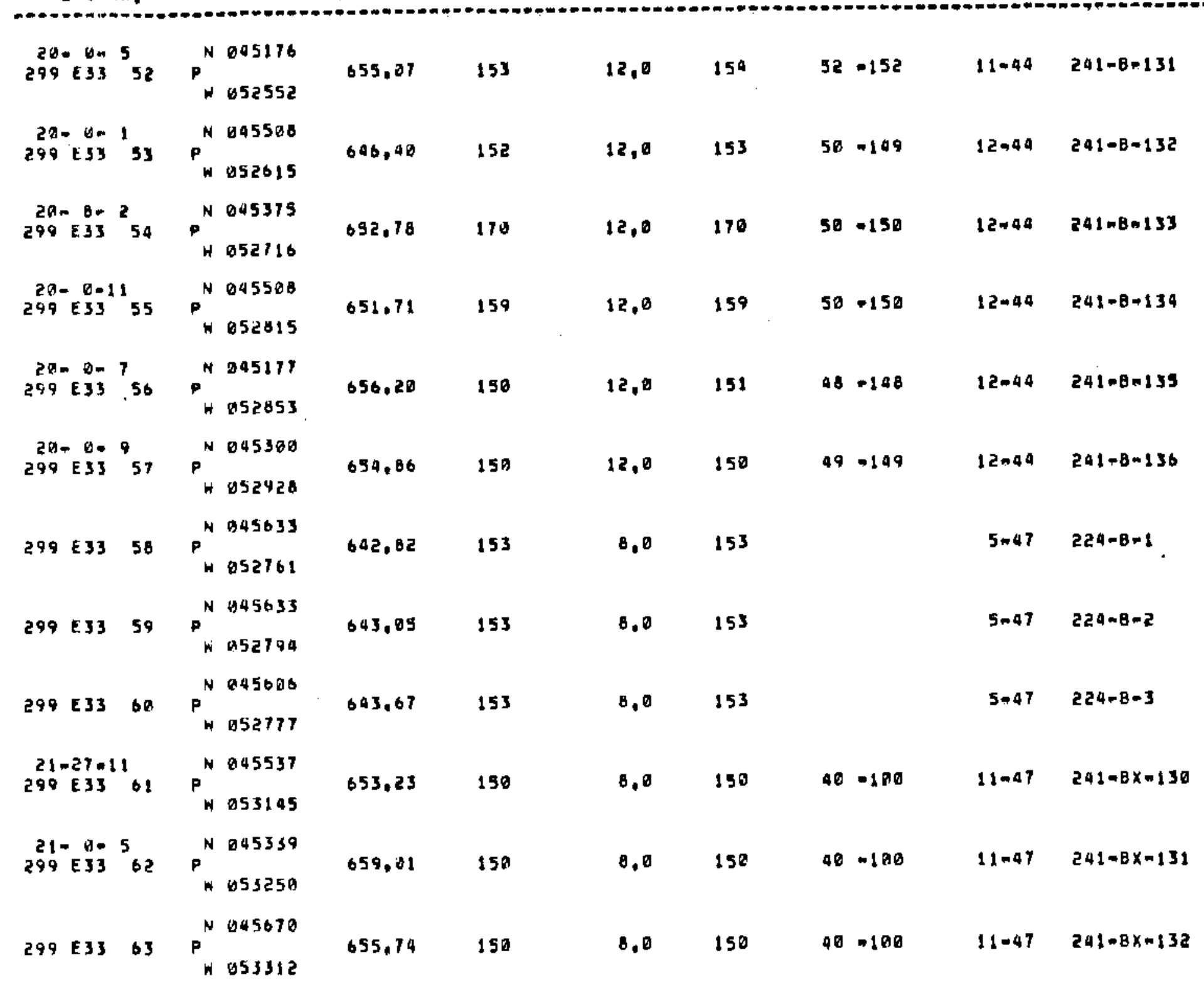




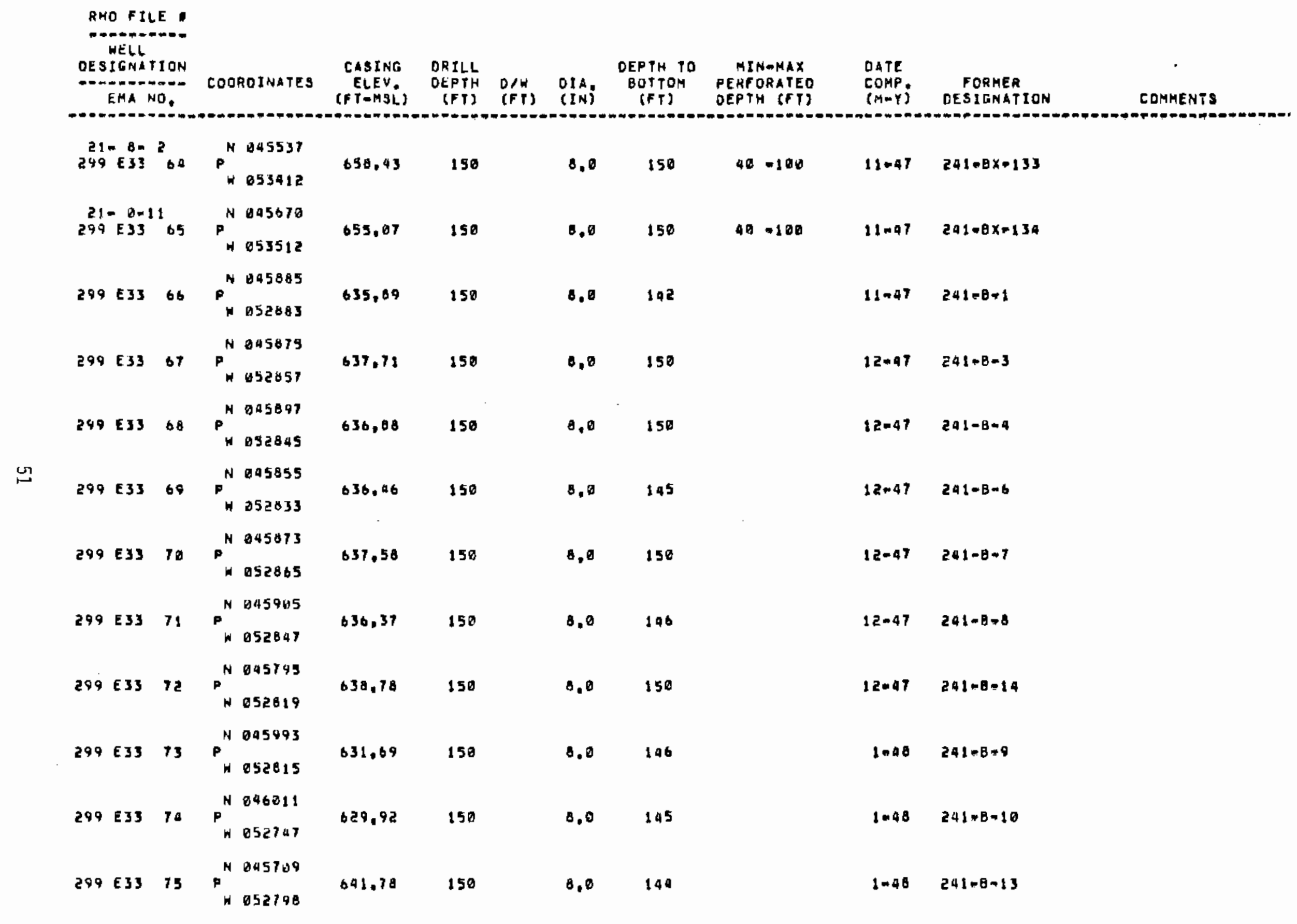




$$
\text { WELL }
$$

DESIGNATION

$$
\text { EMA NOB. }
$$

CODRDINATES

CASING DRILL

FTMSL) DEPTH D/W DIA: BOTTOH PERFORATED

DATE

COMP, FORMER

$(M-Y)$ DESIGNATIOH

COMHENTS

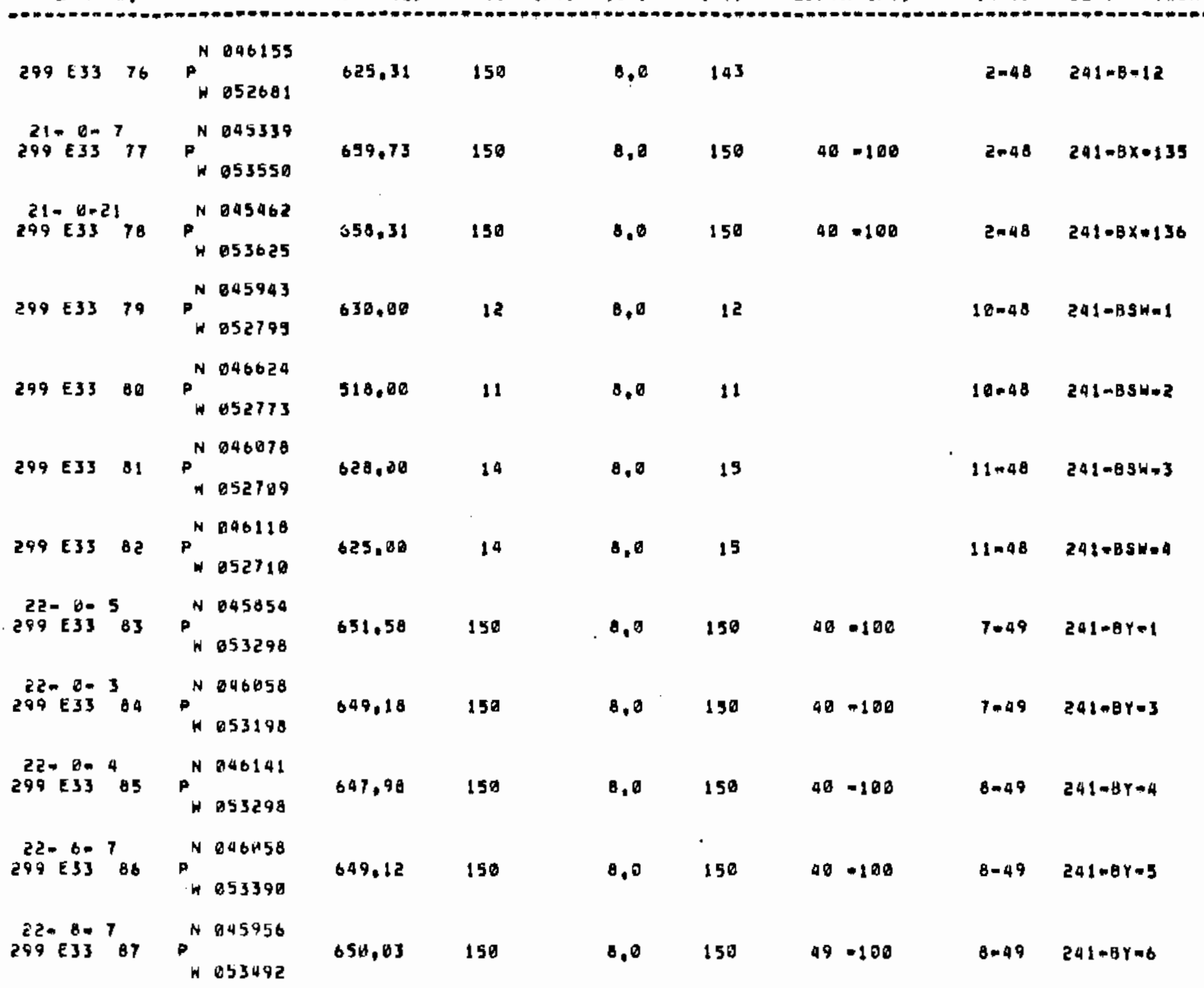


RHO FIle

$$
\text { HELL }
$$

OESIGNATION

OESIGNATION COQROINATES

CASING DRILL (FInABL)

DEPTH

(FT) D/W OIA.

DEPTH TO
BOTOH (FT)
OTTOH

MINAMAX
PERFORATED

DEPTH (FT)

DATE

(H-T)

FORHER

DESIGNATION

COMHENTS

52n H-10 N 046051

$2)^{2}$

$699.00 \quad 150$

8.0150

$48-100$

$0.49 \quad 24108 Y-7$

290 E33 89 N $^{N 095863}$

65,00

150

$8,0 \quad 145$

$12-47 \quad 241-8-2$

$298 E 33 \quad 90$

650.00

141

6.0140

$9-65 \quad E 33+3 A$

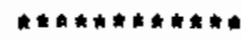

21. 0. 9 N 045462 $299 E 3392$

405623

656,36

75

6,0

$4 \times 67$

$209 E 33^{21 * 0022}$ 93 $P^{N 045464 .}$

656,65

75

6.0

$4 \rightarrow 67$

$25-1203 \quad{ }^{2} 046104$

$299 E 33$ O4 P

$\triangle 48,78 \quad 100$

6.6

$11-\Delta 7$

$22-12=5 \quad N 046061$

$289 \varepsilon 33$ Q5 P
5053532

649,34

6,0

$11-67$

22-12- 6 N 046058

$299 E 33$ 96 P W 253563

$608.40 \quad 100$

$b, 0$

$12-67$

2ट-12-7 N940072

$299 E 33$ 97 P
25053586

$648,15 \quad 100 \quad 6,0$

$11-67$

22-12-9 No46101

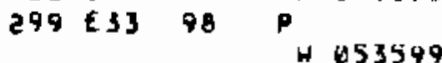

$048,32 \quad 180$

6,0

$12-67$

$22-12-16 \quad N \quad 946142$

$299 E 3399$ P

$647.81 \quad 100 \quad 6.0$

$12=67 \quad$ NO, $6 m 0 Y$ 


\begin{tabular}{|c|c|c|c|c|c|c|c|c|c|}
\hline $\begin{array}{l}\text { WELL } \\
\text { OESIGNATION } \\
\text { EMANO. }\end{array}$ & COOROINATES & $\begin{array}{l}\text { CASING } \\
\text { ELEV. } \\
\text { (FTAHSL) }\end{array}$ & $\begin{array}{l}\text { DRILL } \\
\text { DEPYH } \\
\text { (FT) }\end{array}$ & $\begin{array}{l}D / H \\
(F T)\end{array}$ & $\begin{array}{l}\text { DIA, } \\
\text { (IN) }\end{array}$ & $\begin{array}{l}\text { DEPTH TO } \\
\text { BOTTOH } \\
\text { (FT) }\end{array}$ & $\begin{array}{l}\text { MIN-MAX } \\
\text { PERFQRATED } \\
\text { DEPTH (FT) }\end{array}$ & $\begin{array}{l}\text { DATE } \\
\text { COHP, } \\
(M-Y)\end{array}$ & $\begin{array}{l}\text { FORHER } \\
\text { DESIGNATION COMHENTS }\end{array}$ \\
\hline $\begin{aligned} 22-12 * 1 \\
290 \text { E3 } 10 n\end{aligned}$ & $P_{N 053526}^{N 096138}$ & 648,23 & 100 & & 6.6 & & & $11-67$ & \\
\hline $\begin{array}{rll}22 * & 2.1 \\
299 & 1 \\
293 & 101\end{array}$ & $P_{N 053225}^{N 046038}$ & 648,75 & 100 & & 0.0 & & & $7=70$ & $\begin{array}{l}\text { TANK } 102 \\
\text { NO, } 1\end{array}$ \\
\hline $\begin{array}{rl}220 & 20 \\
299 & 0 \\
35 & 102\end{array}$ & $\begin{array}{l}P^{N} 040005 \\
=053291\end{array}$ & 609,39 & 100 & & 6,6 & & & 8.70 & $\begin{array}{l}\text { TANK } 1 \text { 1月2 } \\
\text { NO. } 3\end{array}$ \\
\hline 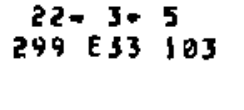 & $P_{N 053223}^{N 046064}$ & 648,30 & 106 & & 6.0 & & & 8.70 & $\begin{array}{l}\text { TANK } 103 \\
\text { NO, } 2\end{array}$ \\
\hline $\begin{array}{rll}22-35 & 1 \\
299 E 33 & 104\end{array}$ & $\begin{array}{l}P^{N} 0463230 \\
05323\end{array}$ & 647,33 & 100 & & 6.0 & & & $8-70$ & $\begin{array}{l}\text { TANK } 103 \\
\text { NO, : }\end{array}$ \\
\hline $\begin{array}{rll}22-35 & 9 \\
299 & 33 & 105\end{array}$ & $P^{N} 046102$ & 648,14 & 160 & & 6,0 & & & $8=70$ & $\begin{array}{l}\text { YANK } 103 \\
\text { NO. } 3\end{array}$ \\
\hline $\begin{array}{lll}22-47 & 1 \\
299 & 533 & 106\end{array}$ & $P_{H}^{N 053327}$ & 649,96 & 106 & & 6,0 & & & $8+70$ & $\begin{array}{l}\text { TANK } 104 \\
\text { NO, } 1\end{array}$ \\
\hline $\begin{array}{rll}22 \times 4 * & 5 \\
299 & 533 & 107\end{array}$ & $P^{N} 045853520$ & 650.01 & 100 & & 0,0 & & & $7 * 70$ & $\begin{array}{l}\text { TANK } 184 \\
\text { NO. } 2\end{array}$ \\
\hline $\begin{array}{rll}220 & 40 & 9 \\
294 & 108\end{array}$ & $\begin{array}{l}P^{N} 045907 \\
4053392\end{array}$ & 649,92 & 168 & & 6.0 & & & 8.70 & $\begin{array}{l}\text { TANX 10a } \\
\text { NO, } 3\end{array}$ \\
\hline $\begin{array}{rll}22-5 \pi & 1 \\
299 & 533 & 109\end{array}$ & $P_{N 053327}^{N 046038}$ & 648,76 & 100 & & 6.0 & & & $7+70$ & $\begin{array}{l}\text { TANK } 165 \\
\text { NO. } 1\end{array}$ \\
\hline $\begin{array}{rll}22 * 5 * 5 \\
299 E 33 & 118\end{array}$ & $\begin{array}{l}P^{N} 045962 \\
N 453327\end{array}$ & 649.69 & 100 & & 6.0 & & & $7-70$ & $\begin{array}{l}\text { TANK } 185 \\
\text { NO. } 2^{165}\end{array}$ \\
\hline $\begin{array}{rll}22-5=9 \\
299 & 533 & 111\end{array}$ & $\begin{array}{l}P^{N} 0408000 \\
{ }_{N}\end{array}$ & 648,96 & 100 & & 6.0 & & & 7.70 & $\begin{array}{l}\text { TanX IAS } \\
\text { NO. } 3\end{array}$ \\
\hline
\end{tabular}




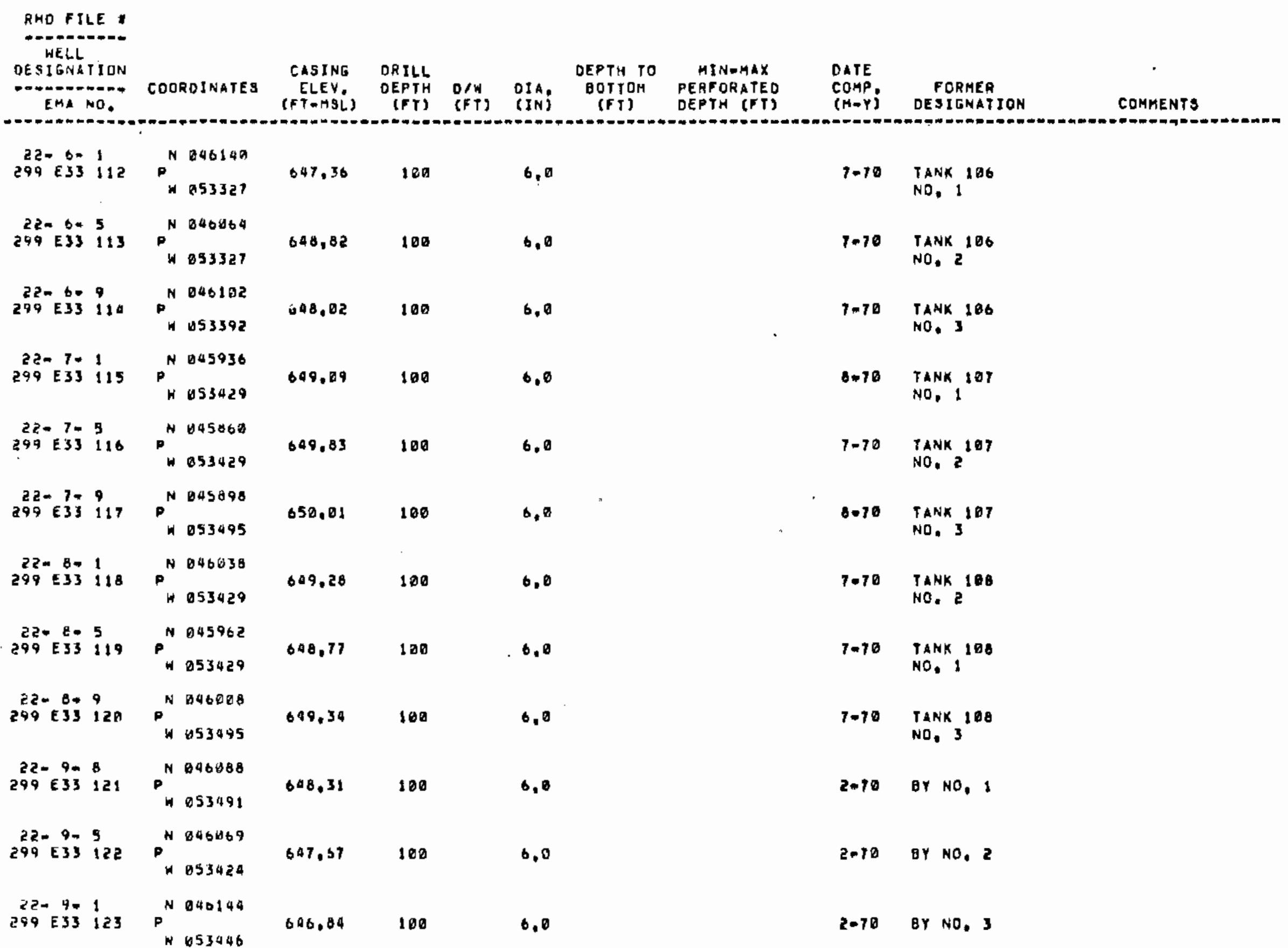




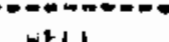

WikLL

DESIGNITIDN

EMA NOA

COORDIHATES

$\begin{array}{lll}\text { CASING DRILL } & & \text { DEPTH TO MINFMAX } \\ \text { ELEV, DEPTH OFH DIA. OOTTOH PERFORATED }\end{array}$

OOTTOH PERFORATED

DATE

COMP

FORMER

DESIGNATION

COMMENTS

\begin{tabular}{|c|c|c|c|c|c|c|c|c|}
\hline $\begin{array}{r}22-1600 \\
299 \text { EJ3 }\end{array}$ & $\begin{array}{l}5 \\
124\end{array}$ & $P_{H}^{N}$ & $\begin{array}{l}045860 \\
053531\end{array}$ & 164.59 & 100 & 6,0 & 8.70 & $\begin{array}{l}\text { TANX } 110 \\
\text { NO, } 2\end{array}$ \\
\hline $\begin{array}{r}22-10-1 \\
290 \text { EJ5 }\end{array}$ & 125 & $P_{H}^{N}$ & $\begin{array}{l}045922 \\
053590\end{array}$ & 649.38 & $18 n$ & 6.0 & $0=10$ & $\begin{array}{l}\text { TANK } 110 \\
\text { ND, } 3\end{array}$ \\
\hline $\begin{array}{r}22-11 \pi \\
299 \text { E33 }\end{array}$ & $\begin{array}{l}1 \\
126\end{array}$ & $P_{W}^{H}$ & $\begin{array}{l}046040 \\
053531\end{array}$ & 649,36 & 100 & 6,8 & 8.70 & $\begin{array}{l}\text { TANX 11! } \\
\text { NO. } 1\end{array}$ \\
\hline $\begin{array}{r}2.2-11= \\
299+33\end{array}$ & $\begin{array}{l}5 \\
127\end{array}$ & $P^{H}$ & $\begin{array}{l}045957 \\
053542\end{array}$ & 649.42 & 100 & 6,0 & 8.70 & $\begin{array}{l}\text { TANK } 111 \\
\text { ND. } 2\end{array}$ \\
\hline $\begin{array}{r}22-110 \\
249 \mathrm{E3} 3\end{array}$ & 9728 & $P_{H}^{N}$ & $\begin{array}{l}040013 \\
053595 .\end{array}$ & 649.30 & 100 & 6.0 & 0.70 & $\begin{array}{l}\text { TANK } 111 \\
\text { NO. } 3\end{array}$ \\
\hline $\begin{array}{l}21 \% \text { 23 } \\
299 \text { E33 }\end{array}$ & 129 & $D^{N}$ & $\begin{array}{l}045530 \\
055220\end{array}$ & 655.18 & 180 & 6,0 & $4-70^{\circ}$ & $\theta x=1$ \\
\hline $\begin{array}{r}21-25 \\
299 \text { E33 }\end{array}$ & 130 & $p^{N}$ & $\begin{array}{l}045463 \\
053212\end{array}$ & 655.75 & 100 & 6,0 & $4-70$ & $8 x=3$ \\
\hline $\begin{array}{r}21 * 2=1 \\
299 \notin 33\end{array}$ & 131 & $P^{N}$ & $\begin{array}{l}045537 \\
653272\end{array}$ & 655,00 & 100 & 6,0 & $4-70$ & $8 x-4$ \\
\hline $\begin{array}{l}21-27-1 \\
290 \mathrm{E} \$ 3\end{array}$ & 132 & $P_{W}^{N}$ & $\begin{array}{l}045524 \\
053183\end{array}$ & 655,40 & 150 & 6.0 & $5 \times 70$ & $\theta x-5$ \\
\hline $\begin{array}{r}21-270 \\
299 \in 33\end{array}$ & 133 & $P^{N}$ & $\begin{array}{l}045490 \\
053160\end{array}$ & 654,53 & 180 & 6,6 & $5-70$ & $B X=6$ \\
\hline $\begin{array}{r}21-27= \\
299 E 33\end{array}$ & $\begin{array}{l}7 \\
134\end{array}$ & $P_{W}^{N}$ & $\begin{array}{l}045459 \\
053169\end{array}$ & 655.36 & 100 & 6,0 & $5-70$ & $B x=7$ \\
\hline $\begin{array}{r}21 * 1= \\
299 \text { E33 }\end{array}$ & $\begin{array}{l}2 \\
155\end{array}$ & $P^{N}$ & $\begin{array}{l}045410 \\
053185\end{array}$ & 650.46 & 108 & 6,0 & $5 \times 70$ & $8 x=8$ \\
\hline
\end{tabular}




\begin{tabular}{|c|c|c|c|c|c|c|c|c|c|c|}
\hline $\begin{array}{c}\text { HELL } \\
\text { OE IGNLTION } \\
\text { EMA NOA. }\end{array}$ & COORDINATES & $\begin{array}{c}\text { CASING } \\
\text { ELEV. } \\
\text { (FT=MSL) }\end{array}$ & $\begin{array}{l}\text { DRILL } \\
\text { DEPIH } \\
\text { (FT) }\end{array}$ & $\begin{array}{l}\mathrm{O} / \mathrm{W} \\
(\mathrm{FT})\end{array}$ & $\begin{array}{l}\text { OIA; } \\
\text { (IN) }\end{array}$ & $\begin{array}{l}\text { DEPTH TO } \\
\text { BOTTOH } \\
\text { (PT) }\end{array}$ & $\begin{array}{l}\text { MINAMAX } \\
\text { PERFOAATED } \\
\text { DEPTH (FT) }\end{array}$ & $\begin{array}{l}\text { DATE } \\
\text { COMP; } \\
\text { (M-Y) }\end{array}$ & $\begin{array}{l}\text { FORMER } \\
\text { DESIGNATION }\end{array}$ & COMHENTS \\
\hline $299 E 33136$ & $\begin{array}{l}P_{N}^{N} 045490 \\
N 05220\end{array}$ & 655.78 & 19 & & 6.0 & & & $.6-70$ & $8 x=9$ & \\
\hline $29 \dot{9} E 3 y 137$ & $\begin{array}{l}P^{N} 045470 \\
k 053249\end{array}$ & 655.49 & 14 & & 6,0 & & & $5=70$ & $8 x-10$ & \\
\hline $\begin{array}{rll}2\}-57 & 2 \\
299^{2} & 133 & 138\end{array}$ & $\begin{array}{l}P_{N}^{N} 045510 \\
N 05120\end{array}$ & 652,21 & 108 & & 0.0 & & & $5-70$ & $6 x-11$ & \\
\hline $\begin{array}{rll}21+277 & 6 \\
299 E 33 & 139\end{array}$ & $\begin{array}{l}P^{N} 045970 \\
W 053120\end{array}$ & 653.88 & 100 & & 6,0 & & & $5=70$ & $8 x-12$ & \\
\hline $\begin{array}{rll}21-00 & 3 \\
299 & E S 3 & 140\end{array}$ & $\begin{array}{l}P_{H}^{N} 045311 \\
H 3140\end{array}$ & 655,06 & 100 & & 6.0 & & & $5-70$ & $8 x-13$ & \\
\hline $\begin{array}{rl}21-27 & 1 \\
299 & 333 \\
141\end{array}$ & $\begin{array}{l}P_{N}^{N} 05312050 \\
N 120\end{array}$ & 651.42 & 100 & & 6,0 & & & $6-70$ & $8 x+14$ & \\
\hline $\begin{array}{rll}21007 & 2 \\
299 & 133 & 142\end{array}$ & $\begin{array}{l}P_{N}^{N} 045570 \\
N 3160\end{array}$ & 652.48 & 100 & & 6.6 & & & 6.70 & $8 x-15$ & \\
\hline $\begin{array}{rl}21-2-2 & 6 \\
299 & =33 \\
103\end{array}$ & $\begin{array}{l}P^{N} 045450 \\
\end{array} 053240$ & 656.88 & 100 & & 0,0 & & & $7 \times 70$ & $8 x=16$ & \\
\hline 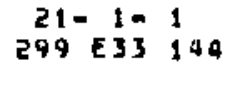 & $\begin{array}{l}P_{N}^{N} 0454300 \\
P_{0} 05220\end{array}$ & 656,29 & 108 & & 6.0 & & & $7-70$ & $8 x-17$ & \\
\hline $\begin{array}{lll}21-20 & 3 \\
299 & 033 & 145\end{array}$ & $\begin{array}{l}P_{N}^{N} 045500 \\
\text { No53208 }\end{array}$ & 655,56 & 100 & & 6.0 & & & 8.78 & $8 x=18$ & \\
\hline $\begin{array}{rll}21-27 & 8 \\
299633 & 146\end{array}$ & $\begin{array}{l}{ }_{0}^{N} 005470 \\
N 053195\end{array}$ & 656,11 & 150 & & 6.8 & & & 8.78 & $8 x+19$ & \\
\hline $\begin{array}{c}20 \cdot 7-11 \\
299 E 33^{147}\end{array}$ & $\begin{array}{l}P_{N 045280}^{N 052770} \\
N 00\end{array}$ & 653.63 & 100 & & 6.0 & & & $4-70$ & $B-1$ & \\
\hline
\end{tabular}


RHO FILE :

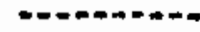

IELI.

DESIGNATION

ExA NOA COOROINATES

EMA NO,

CASING DAILL

ELEY. DEPTH

(FT) O/W DIA, BOTTOM

(FT) (FT)

(IN)

(FT)

MIN-HAX
PERFORATEO
OEPTH (FT)

DATE

COMP, FORMER

(H-Y) DEBIGNATION

COMMENTS

\begin{tabular}{|c|c|c|c|c|c|c|c|c|}
\hline $\begin{array}{rl}290 & 10 \\
299 & \mathrm{E} 33\end{array}$ & 140 & $p^{N}$ & $\begin{array}{l}045229 \\
052795\end{array}$ & 654,48 & 135 & 6,0 & $4-73$ & $\operatorname{Bn} 2$ \\
\hline $\begin{array}{r}20-7= \\
299 \text { E33 }\end{array}$ & 5 & $p^{N}$ & $\begin{array}{l}645195 \\
052734\end{array}$ & 654.89 & 100 & 6.0 & $4-70$ & $B-3$ \\
\hline $\begin{array}{l}21-8-1 \\
299 E 33\end{array}$ & 158 & $P^{M}$ & $\begin{array}{r}045549 \\
053459\end{array}$ & 659.92 & 100 & 6,0 & $12=71$ & \\
\hline $299^{210} 833$ & 65 & $P^{N}$ & $\begin{array}{l}845456 \\
053450\end{array}$ & 655,50 & 100 & 6,0 & $1-72$ & - \\
\hline $\begin{array}{rl}21-80 \\
299 & 033\end{array}$ & $\begin{array}{l}7 \\
152\end{array}$ & $p^{N}$ & $\begin{array}{l}045478 \\
853484\end{array}$ & 655.44 & 100 & 6,0 & $1-12$ & \\
\hline $\begin{array}{r}21+12= \\
299 \mathrm{E3}\end{array}$ & $\begin{array}{l}2 \\
153\end{array}$ & $p^{N}$ & $\begin{array}{l}045616 \\
053503\end{array}$ & 653,82 & 100 & 6.0 & $12=71$ & $\cdot$ \\
\hline $\begin{array}{r}21-12= \\
299 \mathrm{E3} 3\end{array}$ & $\begin{array}{l}5 \\
150\end{array}$ & $P^{N}$ & $\begin{array}{l}045962 \\
053528\end{array}$ & 654.56 & 100 & 6.0 & $11-71$ & \\
\hline $\begin{array}{r}21-12= \\
299 E 33\end{array}$ & 155 & $P^{N}$ & $\begin{array}{l}045569 \\
053581\end{array}$ & 654.90 & 100 & 0.0 & $1+71$ & \\
\hline $\begin{array}{r}21-12-1 \\
299 E 33\end{array}$ & 156 & $P^{N}$ & $\begin{array}{l}045631 \\
053581\end{array}$ & 653,98 & 100 & 6.0 & $12=71$ & \\
\hline $\begin{array}{l}21-5-1 \\
299-[33\end{array}$ & 357 & $P^{N}$ & $\begin{array}{l}045547 \\
053346\end{array}$ & 655.21 & 100 & 6,0 & $12-71$ & \\
\hline $\begin{array}{r}21-5- \\
299 \text { E33 }\end{array}$ & $\begin{array}{l}2 \\
158\end{array}$ & $P^{N}$ & $\begin{array}{l}045531 \\
053319\end{array}$ & 655,02 & 100 & 6,0 & $11-71$ & \\
\hline $\begin{array}{r}21 \cdot 5 n \\
299 \text { E33 }\end{array}$ & $\begin{array}{l}3 \\
159\end{array}$ & $P_{N}^{N}$ & $\begin{array}{l}045590 \\
053304\end{array}$ & 655.44 & 100 & 6.0 & 11.71 & \\
\hline
\end{tabular}




\begin{tabular}{|c|c|c|c|c|c|c|c|c|c|}
\hline $\begin{array}{c}\text { WELL } \\
\text { DESIGNATION } \\
\text { EHANO. }\end{array}$ & COORDINATES & $\begin{array}{c}\text { CASING } \\
\text { ELEY, } \\
\text { (FTAHSL) }\end{array}$ & $\begin{array}{l}\text { DRILL } \\
\text { DEPTH } \\
\text { (FT) }\end{array}$ & $\begin{array}{ll}O / W \\
(F T)\end{array}$ & $\begin{array}{l}\text { OIA; } \\
\text { IIN) }\end{array}$ & $\begin{array}{l}\text { DEPTH TO } \\
\text { BOTTOM } \\
\text { (FT) }\end{array}$ & $\begin{array}{l}\text { MINWAAX } \\
\text { PEAFORATEO } \\
\text { DEPTH (FTS }\end{array}$ & $\begin{array}{l}\text { DATE } \\
\text { COMP, } \\
\text { (M-Y) }\end{array}$ & $\begin{array}{l}\text { FORMER } \\
\text { DESIGNATION COMMENTS }\end{array}$ \\
\hline 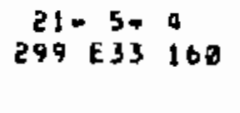 & $\begin{array}{l}P_{N}^{N} 845469 \\
N 3519\end{array}$ & 655,53 & $10 n$ & & 6.0 & & & $11-71$ & \\
\hline $\begin{array}{rl}21 & =5 * 6 \\
299 & E 3\end{array}$ & $\begin{array}{l}P^{N} 045453 \\
N 053346\end{array}$ & 655.93 & 100 & & 6,0 & & & $11-7 \mid$ & \\
\hline $\begin{array}{c}21-5-19 \\
299 E 33162\end{array}$ & $P_{N 053386}^{N 045522}$ & 695.06 & 100 & & 6.0 & & & $12-7 \mid$ & \\
\hline $\begin{array}{rl}21-00 & 1 \\
299 E 3] & 163\end{array}$ & $\begin{array}{l}P^{N} 045644 \\
N 053346\end{array}$ & 653,75 & 100 & & 6.8 & & & $12-11$ & \\
\hline $\begin{array}{rll}2106 & 6 \\
299 & 33 & 164\end{array}$ & $\begin{array}{l}P^{N} 045631 \\
N 053319 .\end{array}$ & 653,93 & 100 & & 6.8 & & & $12-71$ & \\
\hline 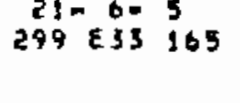 & $P^{N} 05535319$ & 654,70 & 100 & & 6,0 & & & $12-71$ & \\
\hline $\begin{array}{c}21 m 00-10 \\
290^{\circ} E 33166\end{array}$ & $P_{H}^{N 0533866}$ & 653.67 & 100 & & 0,0 & & & 12.71 & • \\
\hline $\begin{array}{rl}21-100 & 1 \\
249 \text { E33 } 167\end{array}$ & $\begin{array}{l}P^{N} 045432 \\
N 053519\end{array}$ & 056.12 & 100 & & 0.0 & & & 9.71 & \\
\hline $\begin{array}{rll}21-10 & 5 \\
299 E 33 & 108\end{array}$ & $\begin{array}{l}P^{N} 045369 \\
05319\end{array}$ & 656,51 & 100 & & 6.0 & & & $9 \cdot 71$ & \\
\hline 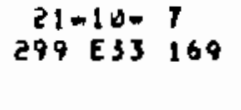 & $\begin{array}{l}P^{N} 045369 \\
N 053581\end{array}$ & 656.68 & 100 & & 6.0 & & & $9-11$ & \\
\hline $\begin{array}{rl}21-10-11 \\
299 & 33170\end{array}$ & $P^{N} 045431$ & 650.40 & 100 & & 6,0 & & & $9=71$ & \\
\hline $\begin{array}{rll}21-11 & 5 \\
299 E 33 & 171\end{array}$ & $P_{W}^{N} 055519$ & 655.62 & 100 & & 6.0 & & & $9 \times 71$ & \\
\hline
\end{tabular}


NELL

OESIENATION

EHA Ain

CDOADINATES

CASING

ORILL

O/H

DIA.

DEPTH TO

OTTOHO MINAMAX

(Fi)

PERFURATED

DEPTH (FT)

DATE

COMP. FORMEA

(HAY) DESIGNATION

CDMMENTS

$21-11 n, 7$ N 845461

$21-11 \cdots 7$

P

$650.21 \quad 100$

$B, 0$

$9-71$

$21-11=4 \quad N 645478$

$299 E 33173$

$\times 053512$

$655.32 \quad 100$

6.0

$9-71$

$21-11-10$

N 045522

$299 E 3517 a$

$p$. 045525

$655.49 \quad 100$

6.0

$9+71$

$29-11+11$ E 2045532

$655,34 \quad 109$

6.0

$8=71$

$22=1-1 \quad N 045929$

290633176

$049,78 \quad 100$

6.0

12072

g

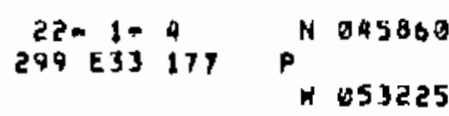

$650.59 \quad 100$

$B, 0$

$|z-7|$

22- 1*T N 045867

$650.76 \quad 100$

6,6

$12-71$

290 E33 179 P $P^{N 045357}$

$692,65 \quad 100$

6.0

$2-72$

20-2. 5 N 145296

$290 E 33180 \quad P_{\text {H H52543 }}$

$652.58 \quad 140$

0.6

$5=73$

20. $2.7 \quad N 005300$

$299 E 33181$

$P$ OS2575

$652.62 \quad 100$

6.0

3-72

20-20 $9 \quad N 645337$

$\begin{array}{lll}299 & 233 & 182\end{array}$

w 052598

$652.81 \quad 100$

6.0

$2=72$

$20-2011=$

N

$652.39 \quad 100$

0,0

$2-71$ 


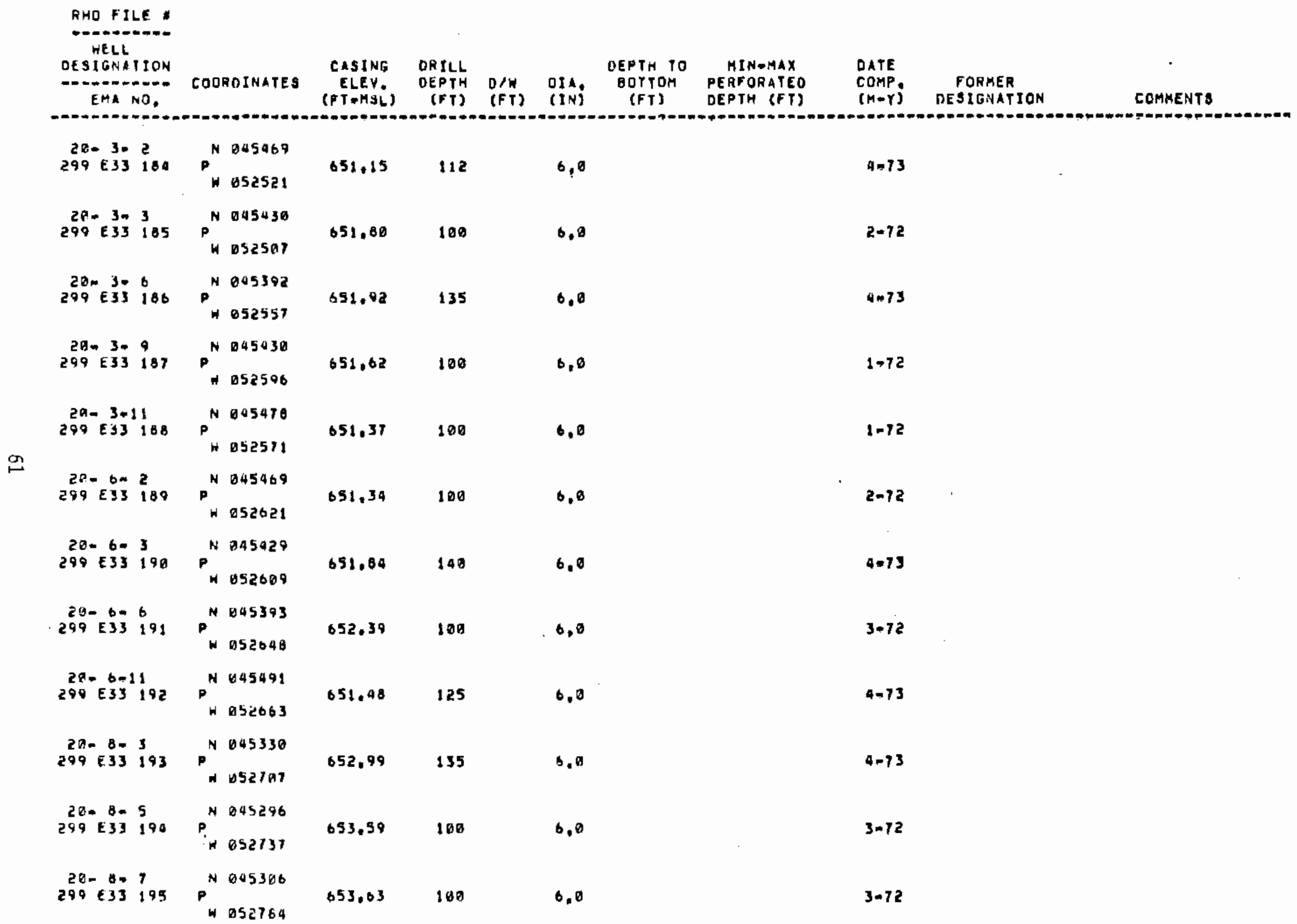




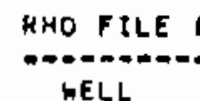

MELL

DESIGNATION

EMA NO.

COOROINATES

CASING DRILL

$\begin{array}{llll}\text { CASING DRILL } & & \text { DEPTH TO MINAMAX } \\ \text { ELEY, DEPTM DIW OIAB BOTTOM PERFORATEO }\end{array}$

DATE

COMP, FORMER

(M-Y) DESIGNATION

COMHENTS

\begin{tabular}{|c|c|c|c|c|c|c|c|}
\hline $\begin{array}{l}200 \\
299 \mathrm{E} 33\end{array}$ & 996 & $P^{N}$ & $\begin{array}{l}049338 \\
052798\end{array}$ & 653.34 & 130 & 6.0 & 4.73 \\
\hline $\begin{array}{l}28-9- \\
299 \text { E35 }\end{array}$ & $\begin{array}{l}2 \\
197\end{array}$ & $P_{H}^{N}$ & $\begin{array}{r}045467 \\
652719\end{array}$ & 651,74 & 100 & 6,0 & 1.72 \\
\hline $\begin{array}{r}29-90 \\
299 E 33\end{array}$ & $\begin{array}{l}6 \\
198\end{array}$ & $P^{N}$ & $\begin{array}{l}045394 \\
052748\end{array}$ & 652,71 & 100 & 0.0 & +1 \\
\hline $\begin{array}{r}20-12 \% \\
289 E 33\end{array}$ & $\begin{array}{l}3 \\
199\end{array}$ & $P^{N}$ & $\begin{array}{l}045438 \\
05246\end{array}$ & 652.03 & 100 & 6,0 & $1-72$ \\
\hline $\begin{array}{r}29-901 \\
299<33\end{array}$ & 21 & $P^{N}$ & $\begin{array}{r}045472 \\
052775 .\end{array}$ & 651.64 & 100 & 6.6 & 972 \\
\hline $\begin{array}{r}20-12= \\
299 E 53\end{array}$ & $\begin{array}{l}2 \\
201\end{array}$ & $P^{N}$ & $\begin{array}{l}645469 \\
652821\end{array}$ & 651.78 & 125 & 6,0 & $4 m 73$ \\
\hline $\begin{array}{r}20-120 \\
299 \mathrm{ES3}\end{array}$ & $\begin{array}{l}6 \\
202\end{array}$ & $P^{N}$ & $\begin{array}{l}045398 \\
052840\end{array}$ & 652,64 & 180 & 6,0 & $1=7$ \\
\hline $\begin{array}{r}27-12- \\
299+33\end{array}$ & $\begin{array}{l}7 \\
203\end{array}$ & $P^{N}$ & $\begin{array}{l}045401 \\
052884\end{array}$ & 652,53 & 109 & 6.0 & $1-72$ \\
\hline $\begin{array}{c}29-12=1 \\
299 E 33\end{array}$ & 204 & $P^{N}$ & $\begin{array}{r}045469 \\
052084\end{array}$ & 652.11 & 100 & 6.0 & $1-72$ \\
\hline $\begin{array}{r}22 * 7 * \\
299 E 33\end{array}$ & $\begin{array}{l}2 \\
206\end{array}$ & $P^{N}$ & $\begin{array}{l}045920 \\
053412\end{array}$ & 649.52 & 106 & 6.0 & 1.73 \\
\hline $\begin{array}{rl}22-80 \\
299 & 633\end{array}$ & 207 & $P^{N}$ & $\begin{array}{l}005454 \\
053449\end{array}$ & 648.84 & 100 & 6.0 & $1-73$ \\
\hline $\begin{array}{r}22-8= \\
298 \text { E33 }\end{array}$ & $\begin{array}{l}2 \\
208\end{array}$ & $P^{N}$ & $\begin{array}{l}046022 \\
053411\end{array}$ & 648,55 & 100 & 6.0 & -73 \\
\hline
\end{tabular}




\begin{tabular}{|c|c|c|c|c|c|c|c|c|c|}
\hline $\begin{array}{l}\text { HELL } \\
\text { DESIGNATION } \\
\text { EHA NO: }\end{array}$ & COOROINATES & $\begin{array}{c}\text { CASING } \\
\text { ELEY, } \\
\text { (FTRMSL) }\end{array}$ & $\begin{array}{l}\text { DAILL } \\
\text { DEPYH } \\
\text { (FT) }\end{array}$ & (F) & DIA: & $\begin{array}{l}\text { DEPTH TO } \\
\text { BDTTOH } \\
\text { (FT) }\end{array}$ & $\begin{array}{l}\text { MINGHAX } \\
\text { PERFORATEO } \\
\text { DEPTH (FT) }\end{array}$ & $\begin{array}{l}\text { DATE } \\
\text { COMPP, } \\
\text { (M-Y) }\end{array}$ & $\begin{array}{l}\text { FORMER } \\
\text { OESIGNATIOH }\end{array}$ \\
\hline $\begin{array}{c}22-8+12 \\
299 E 33 \text { 209 }\end{array}$ & $P^{N 040009}$ & & 106 & & 6,0 & & & 1073 & \\
\hline $\begin{array}{rll}25-30 & 6 \\
299 & \text { ESS } 210\end{array}$ & $P^{N 0460056}$ & & 100 & & 6,0 & & & $12-72$ & \\
\hline $\begin{array}{lll}22-33 & 4 \\
299 & 33 & 211\end{array}$ & $\begin{array}{l}P_{N}^{N 040081} \\
\text { No53207 }\end{array}$ & 608.01 & 100 & & 6,6 & & & $12=72$ & \\
\hline 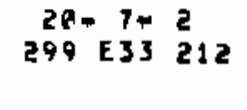 & $\begin{array}{l}P^{N} 045297 \\
W 052715\end{array}$ & 654.25 & 160 & & 6.0 & . & & $8-73$ & \\
\hline $\begin{array}{rll}20-160-2 \\
299 E 33 & 213\end{array}$ & $\begin{array}{l}P_{H}^{N} 045257 \\
H 052815\end{array}$ & 659.02 & 100 & & 6,6 & & & $8-73$ & \\
\hline $\begin{array}{c}20-10-12 \\
299 E 33 \\
214\end{array}$ & $\begin{array}{l}P^{N} 045280 \\
\\
M 52055\end{array}$ & 654,27 & 100 & & 6,0 & & & 8.73 & \\
\hline $\begin{array}{c}20-10-9 \\
299 \text { E33 } 215\end{array}$ & $\begin{array}{l}P^{N} 645238 \\
N 052895\end{array}$ & 695,15 & 135 & & 6.0 & & & 9.73 & \\
\hline $\begin{array}{rll}2 A-10 * & 7 \\
299 E 33 & 216\end{array}$ & $\begin{array}{l}P_{N}^{N} 045200 \\
N 2477\end{array}$ & 655.49 & 160 & & 6.0 & & & 9.73 & \\
\hline $\begin{array}{c}20-11 * 9 \\
299 \text { ES3 } 217\end{array}$ & $P^{N 045337}$ & 653.17 & 126 & & 6.8 & & & 5.73 & \\
\hline $\begin{array}{rll}20 \pi 5 & 50 \\
290 & E 33 & 218\end{array}$ & $P^{N} 045290$ & 653.69 & 120 & & 6,0 & & & 5.73 & \\
\hline $\begin{array}{rll}29-45 & 5 \\
299 & E 33 & 210\end{array}$ & $\begin{array}{l}p^{N} 045237 \\
W 052049\end{array}$ & 659.46 & 100 & & 6,0 & & & 5.73 & \\
\hline $\begin{array}{lll}29-1-3 \\
259 \text { E33 } & 220\end{array}$ & $\begin{array}{l}\text { N } 0452.37 \\
W \text { O52502 }\end{array}$ & 653,03 & 135 & & 6,0 & & & $5=73$ & \\
\hline
\end{tabular}


RHO FILE

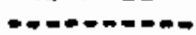

WELL

DESIGHATION

EMA ND, COORDINATES

CASING DRILL

ELEY. DEPTH D/W DIA, DETTOM GOTTOH

MINDHAX

PERFORATED

$\underset{(M+Y)}{C O M P,}$

FORMER

DESIGNATION

COMHENTS

$200406 \quad$ N 895187

W052652

$655.19 \quad 135$

6.0

6.73

$21-7=6$

N 045355

$299 E 53222$

P. 053450

656.79

100

6.0

$9 \times 73$

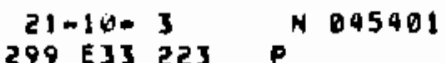

$350.23 \quad 100$

6,0

$9+73$

$299 E 33$ 224 P

$656,67 \quad 100$

6,0

10.73

$21-7=3 \quad N 045398$

299E33 225 P H 153994

$656.08 \quad 100 \quad 6,0$

$10-73$

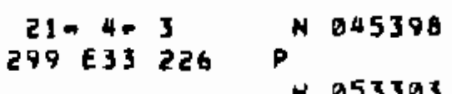

$656,40 \quad 100$

6.0

$10-13$

22- 2- 2 N 946415

289 E3J 257 P H O53203

$654.05 \quad 100 \quad 6,0$

$10-73$

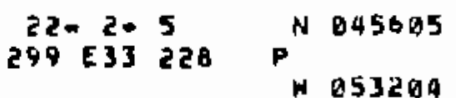

$659.59 \quad 106$

6,0

$10-73$

21-3-5 N 845558

$299 E 33 \quad 229$

$p^{N} 845558$

$655.11 \quad 100$

0.0

$11-73$

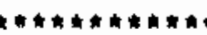

$31-9-12$

N. 045695

P. 053452

$653,70 \quad 100$

6,0

$10-73$

2) $-12-12$

p 245645

H.053552

$653.78 \quad 100$

6,0

$10-73$ 


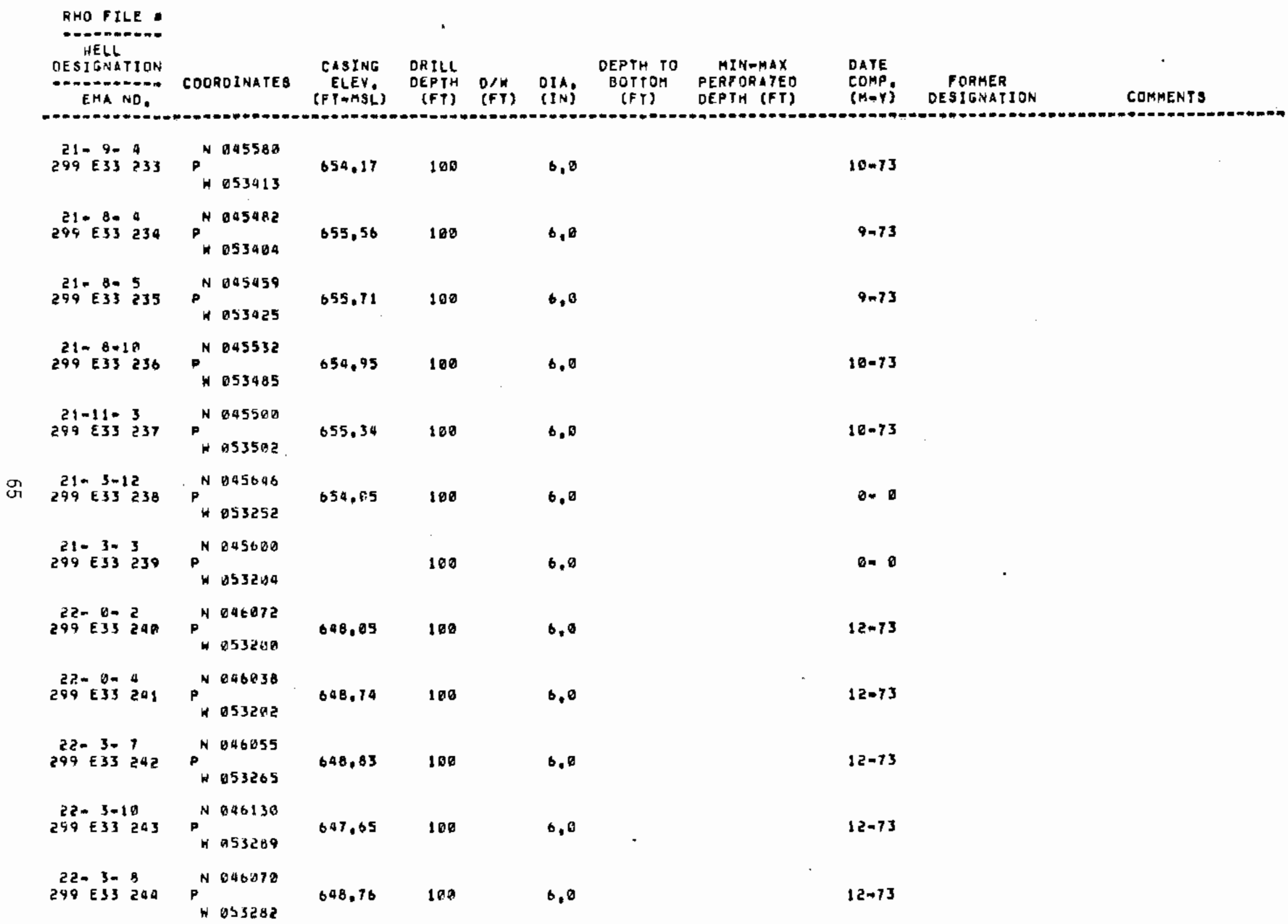


AHO PILE:

NELL

OESIGNATION

OE-Dignarion

COOROINATES CASING

DRILL
OEPTH DIN OIA, BEPTH TO MITOM PERFORATED

DATE

EMA NO,

(F T M MSL)

(FT) (FT)

(IN) (FT)

DEPTH (FT)

$(M=Y)$

FORMER

DESIGNATION

COMMENT 5

\begin{tabular}{|c|c|c|}
\hline $\begin{array}{lll}22 m & 1+3 \\
299 & E 33 & 245\end{array}$ & $0^{N}$ & $\begin{array}{l}045900 \\
053202\end{array}$ \\
\hline $\begin{array}{c}22-1=16 \\
299 E 33246\end{array}$ & $P_{n}^{N}$ & $\begin{array}{l}045911 \\
053292\end{array}$ \\
\hline
\end{tabular}

100

6.0

$5-74$

$299 \quad 633 \quad 247$

100

6,0

$5+79$

0. 6

2द्यन 7 N 045065

$299 E 33248$ P

160

6,0

$5-79$

22-4-11 N 045940

299833249

$P^{N} 005940$
$W 053365$

120

6,8

$5-74$

कू $22-6-11$

N 046140

W 053375

100

6,0

$5=74$

$290 \times 33251 \quad P^{N 045060}$

H. 953481

100

$$
6,0
$$

$5 n 74$

22- 7-10 N 045932

299633252

W 053497

100

6.0

$5=14$

$299 E 33253 P^{N 046137}$

W 053477

100

$$
0.0
$$

$5-74$

22-19.7

299 E33 254

N 045060

W 053577

100

$$
b, 0
$$

$5=79$

$22-16=9$

N 045080

299 E33 255

P DS5600

100

$$
b, 0
$$

$5=74$

$22-11 *$

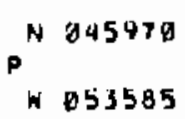




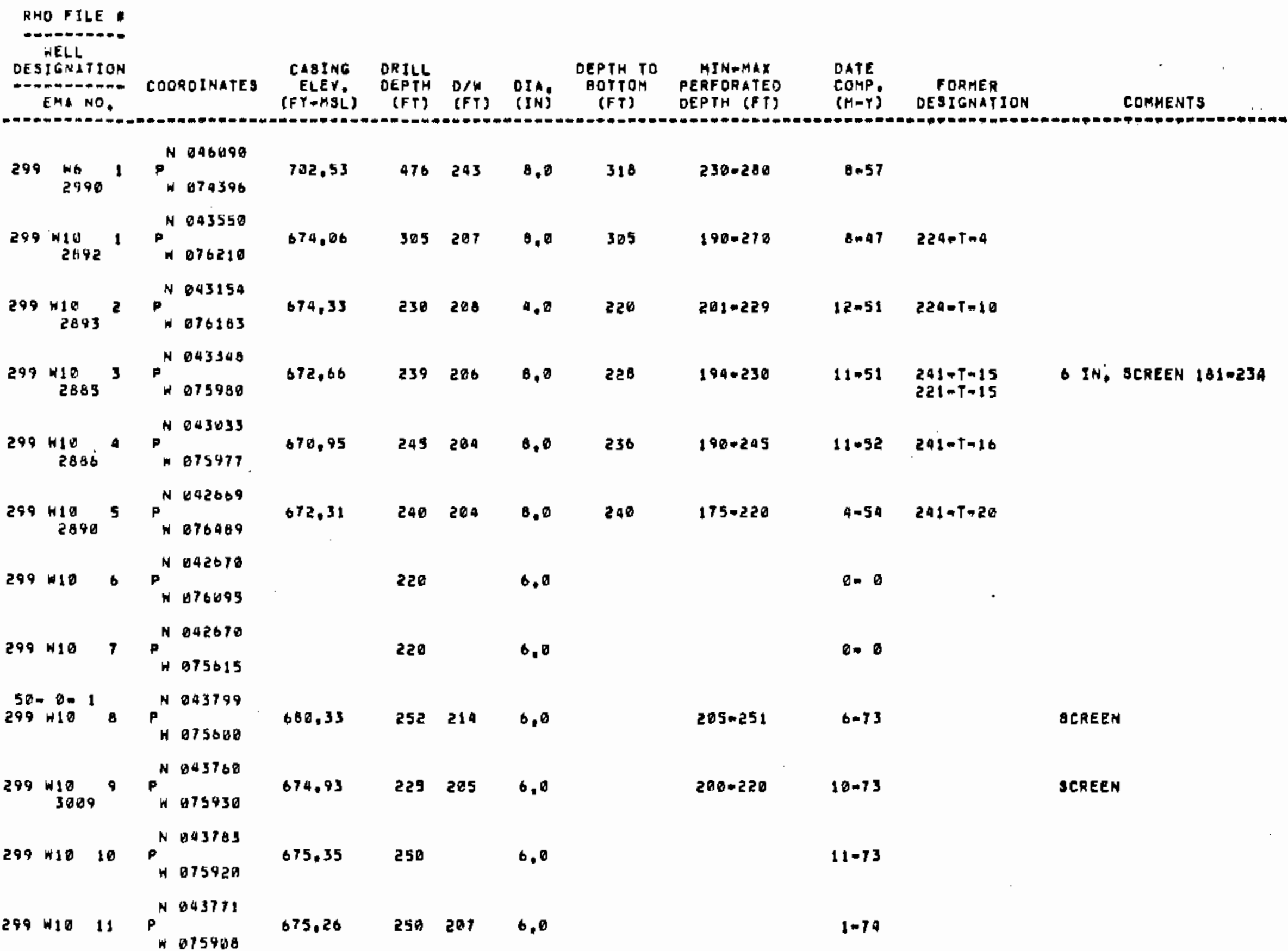




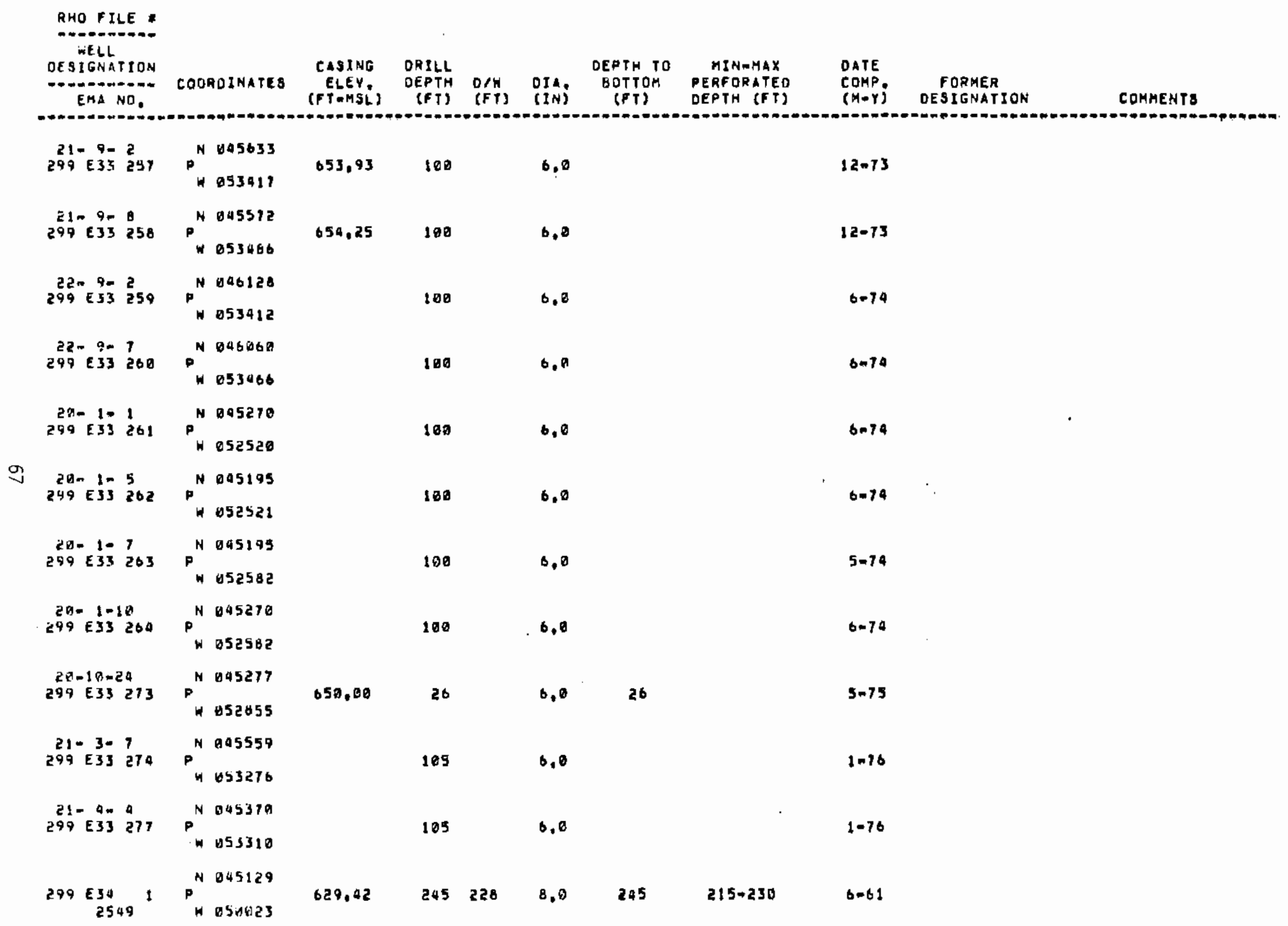




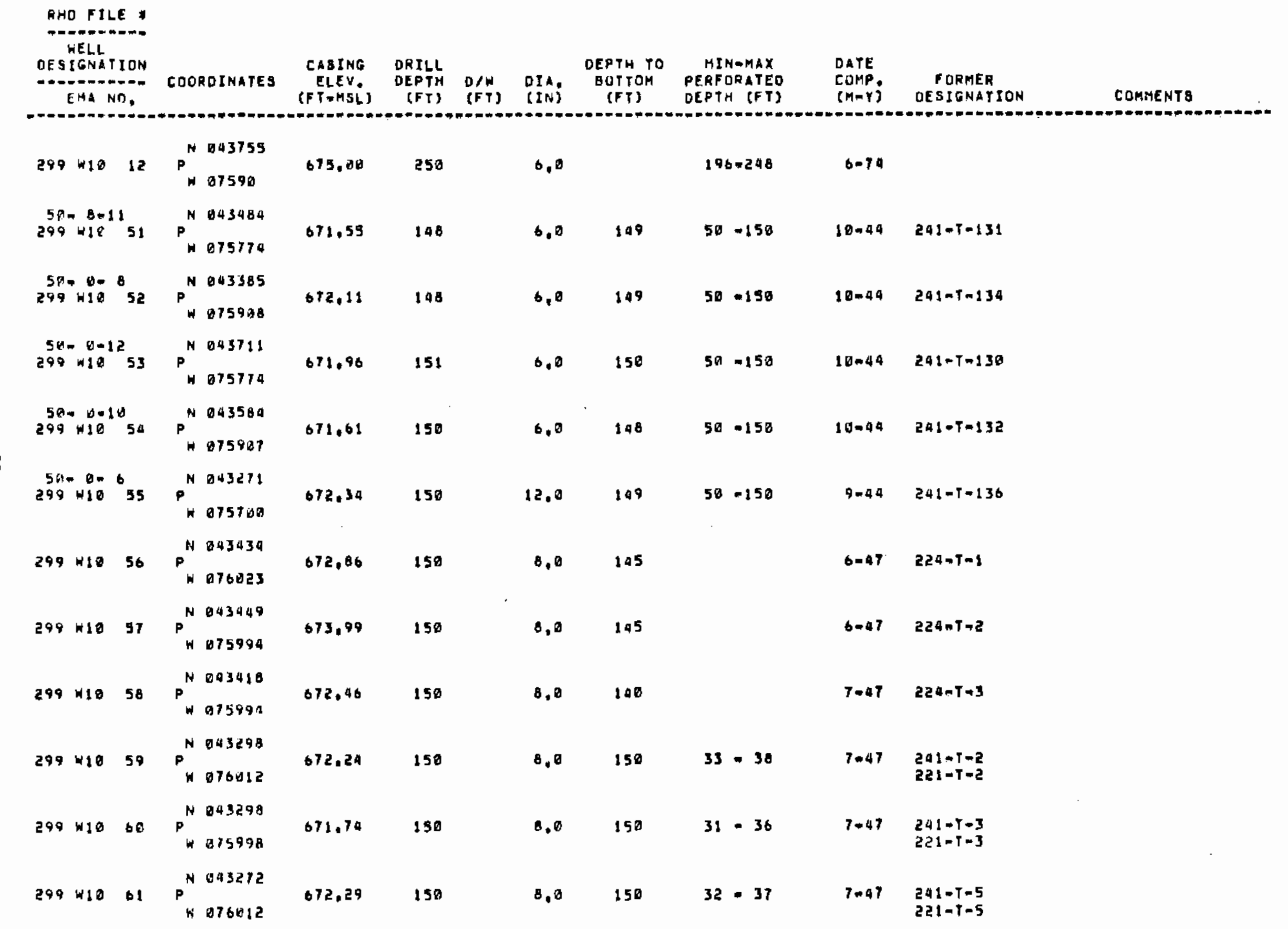




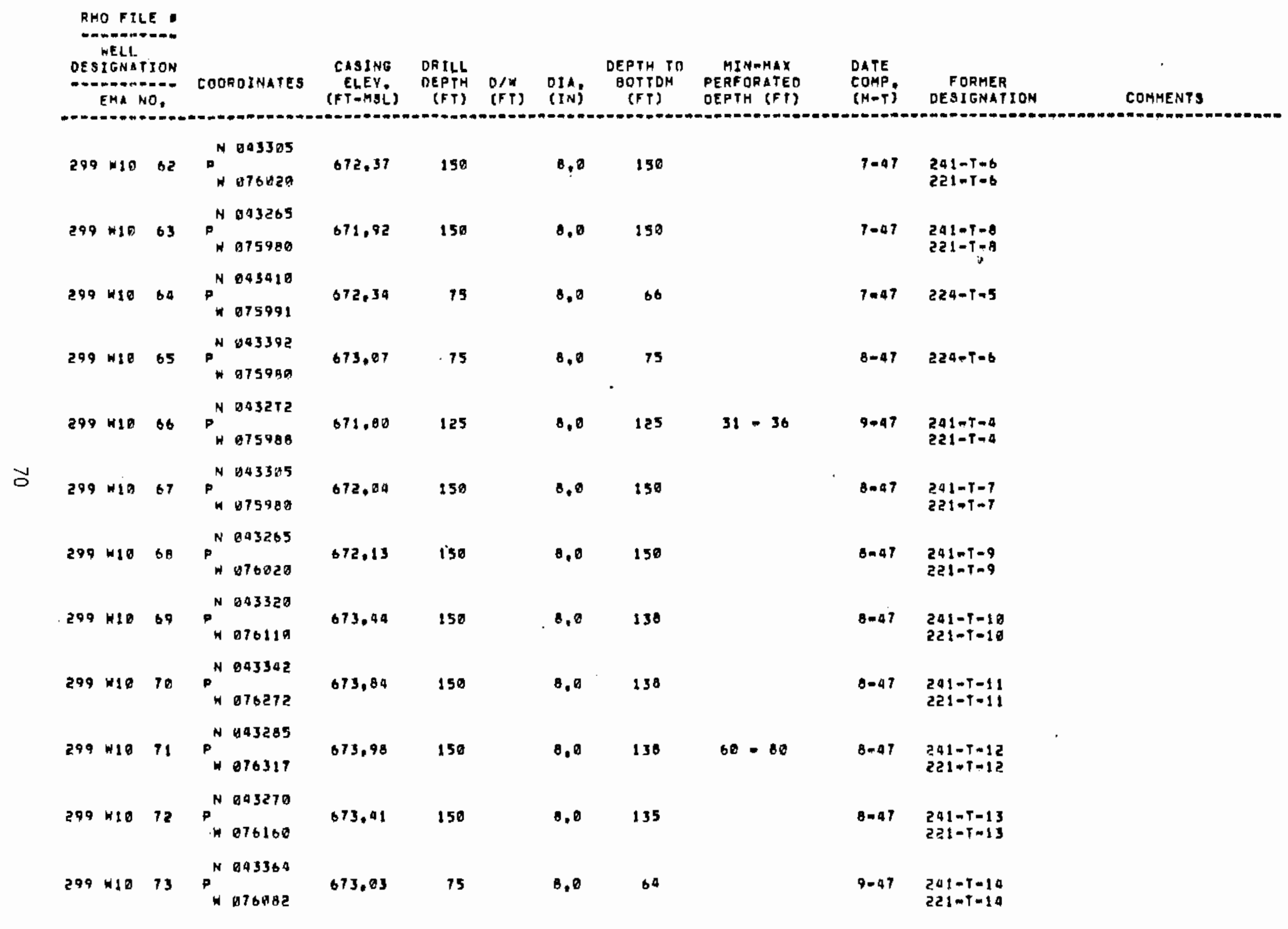




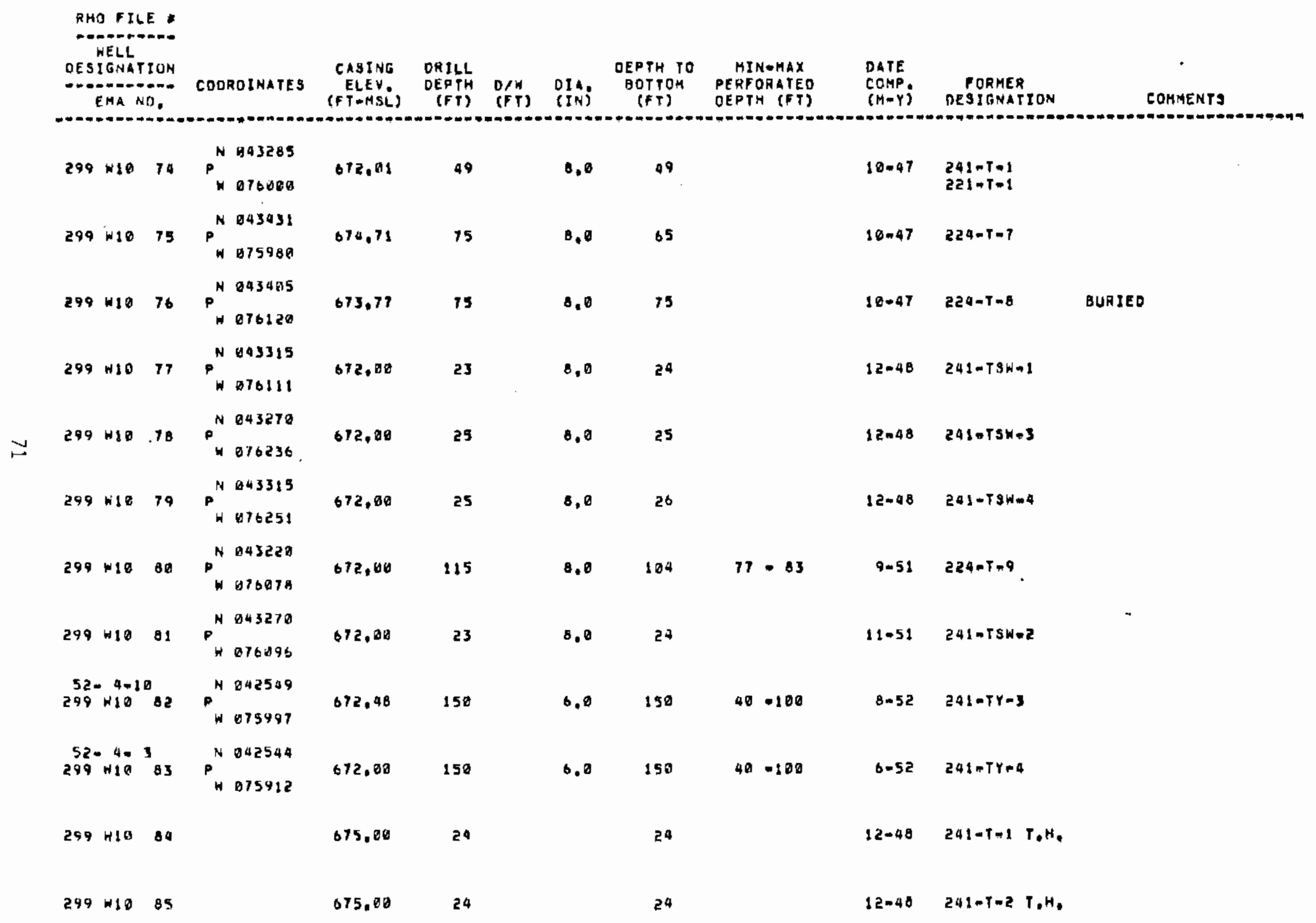




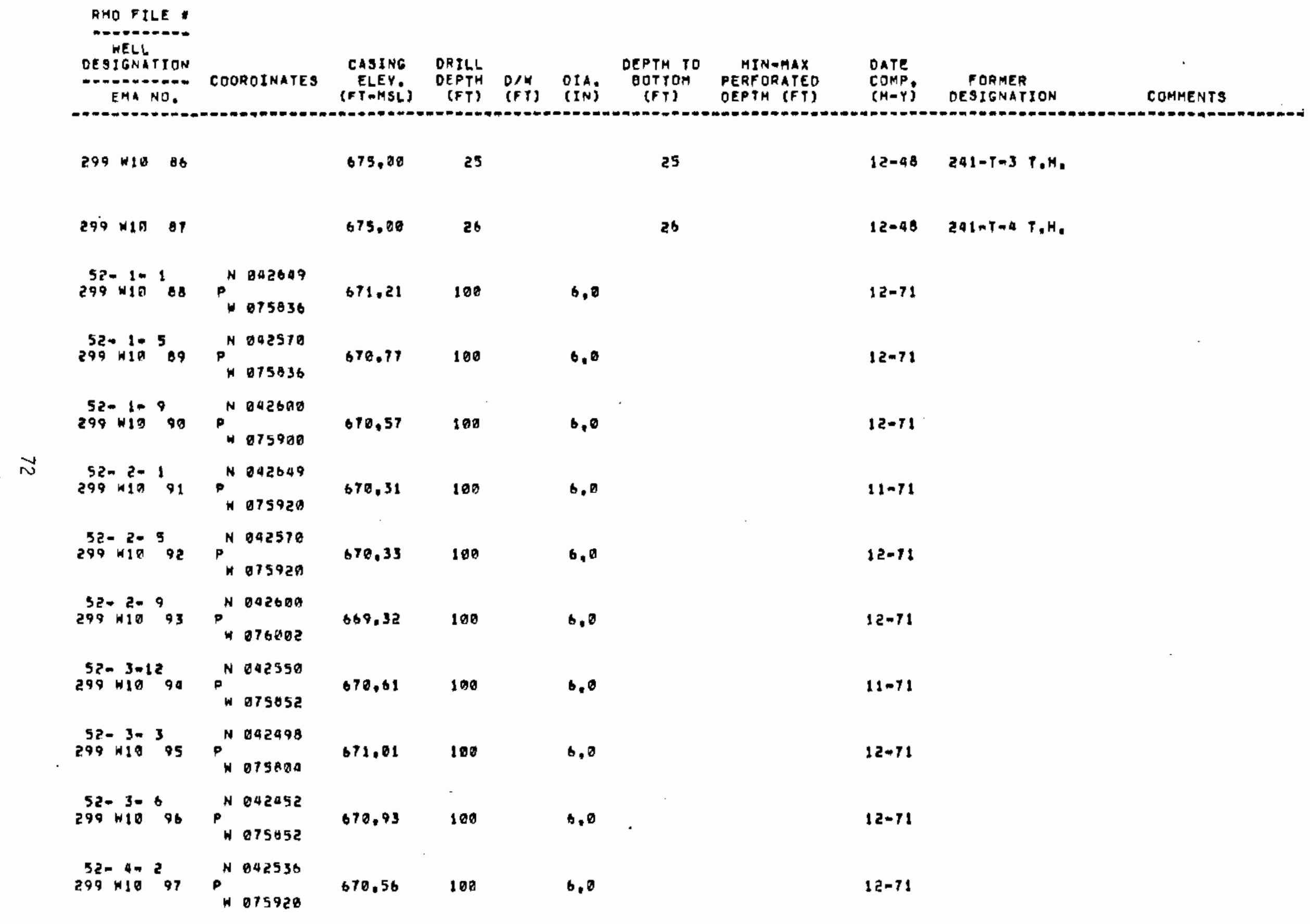




\begin{tabular}{|c|c|c|c|c|c|c|c|c|c|c|}
\hline $\begin{array}{c}\text { NELL } \\
\text { OESIGNATION } \\
\text { EMA NO }\end{array}$ & CODROINATES & $\begin{array}{c}\text { CASING } \\
\text { ELEV. } \\
\text { (FT=MSL) }\end{array}$ & $\begin{array}{l}\text { DRILL } \\
\text { DEPTH } \\
\text { (FY) }\end{array}$ & (F) & OIA; & $\begin{array}{l}\text { OEPTH TO } \\
\text { BOTTOH } \\
(F T)\end{array}$ & $\begin{array}{l}\text { MIN-MAX } \\
\text { PERFORATED } \\
\text { OEPTH (FT) }\end{array}$ & $\begin{array}{l}\text { OATE } \\
\text { COMP } \\
\text { (M-Y) }\end{array}$ & $\begin{array}{l}\text { FORMER } \\
\text { DESIIGNATION }\end{array}$ & COMHENTS \\
\hline $\begin{array}{l}52-90^{\circ} \\
299\end{array}$ & $\begin{array}{l}P_{N}^{N} 042452 \\
N 6594\end{array}$ & 670.21 & 100 & & 6.0 & & & $12-71$ & & \\
\hline $\begin{array}{r}52-40^{\circ} \\
299 \times 10^{9}\end{array}$ & $\begin{array}{l}P_{H}^{N} 0425060042 \\
{ }^{2}\end{array}$ & 069.30 & 100 & & 6.0 & & & $12-71$ & & \\
\hline $\begin{array}{lll}52-6= & 2 \\
299 & 100 & 100\end{array}$ & $\begin{array}{l}P_{N}^{N} 042443 \\
N \quad 75928\end{array}$ & 670,57 & 60 & & 6.8 & & & B. 0 & & \\
\hline 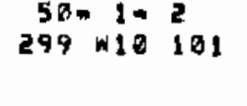 & $\begin{array}{l}P_{N}^{N} 043671 \\
N_{07597}\end{array}$ & 674,31 & 9 a & & 6.8 & & & $7-73$ & & \\
\hline $\begin{array}{rll}580+10 & 4 \\
299 & 10 & 102\end{array}$ & $\begin{array}{l}P_{W 075595}^{N} 043632 \\
W 075\end{array}$ & 674,18 & 87 & & 6.0 & & & $8-73$ & & \\
\hline $299 \times 10103$ & $\begin{array}{l}P^{N} 075057 \\
0073\end{array}$ & 673.04 & 94 & & 6.8 & & & $8-73$ & - & \\
\hline $\begin{array}{rl}58-109 \\
298+18 & 180\end{array}$ & $\begin{array}{l}P_{H}^{N} 843685 \\
H 075680\end{array}$ & 673.17 & 96 & & 6,8 & & & $8-73$ & & \\
\hline $\begin{array}{c}58 \cdot 1-12 \\
278+10105\end{array}$ & $\begin{array}{l}P_{N}^{N} 403692 \\
H 075037\end{array}$ & 673.46 & 92 & & 6.8 & & & $7-73$ & & \\
\hline $\begin{array}{rl}50-60 & 6 \\
299 & \text { wid } 106\end{array}$ & $\begin{array}{l}P^{N} 843502 \\
N 07503 \%\end{array}$ & 671,42 & 100 & & 6.0 & .123 & & $7-13$ & & DEEPENEO $3-77$ \\
\hline 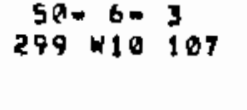 & $\begin{array}{l}P^{N} 043535 \\
H \quad 075792\end{array}$ & 671,51 & 100 & & 6,0 & $\$ 22$ & & $7 m 73$ & & DEEPENED $3=77$ \\
\hline $\begin{array}{rl}50-60 & 2 \\
299+10 & 188\end{array}$ & $\begin{array}{l}P^{N} 0435800 \\
\cdot 075845\end{array}$ & 671.84 & 92 & & 0.0 & 125 & & $7=73$ & & DEEPENED $\quad 5 \rightarrow 77$ \\
\hline $\begin{array}{c}58-0 * \\
299 \\
29\end{array}$ & 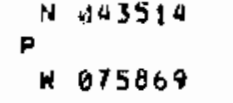 & 671.10 & 92 & & 6.0 & 123 & & $7-73$ & & DEEPENED 3.77 \\
\hline
\end{tabular}




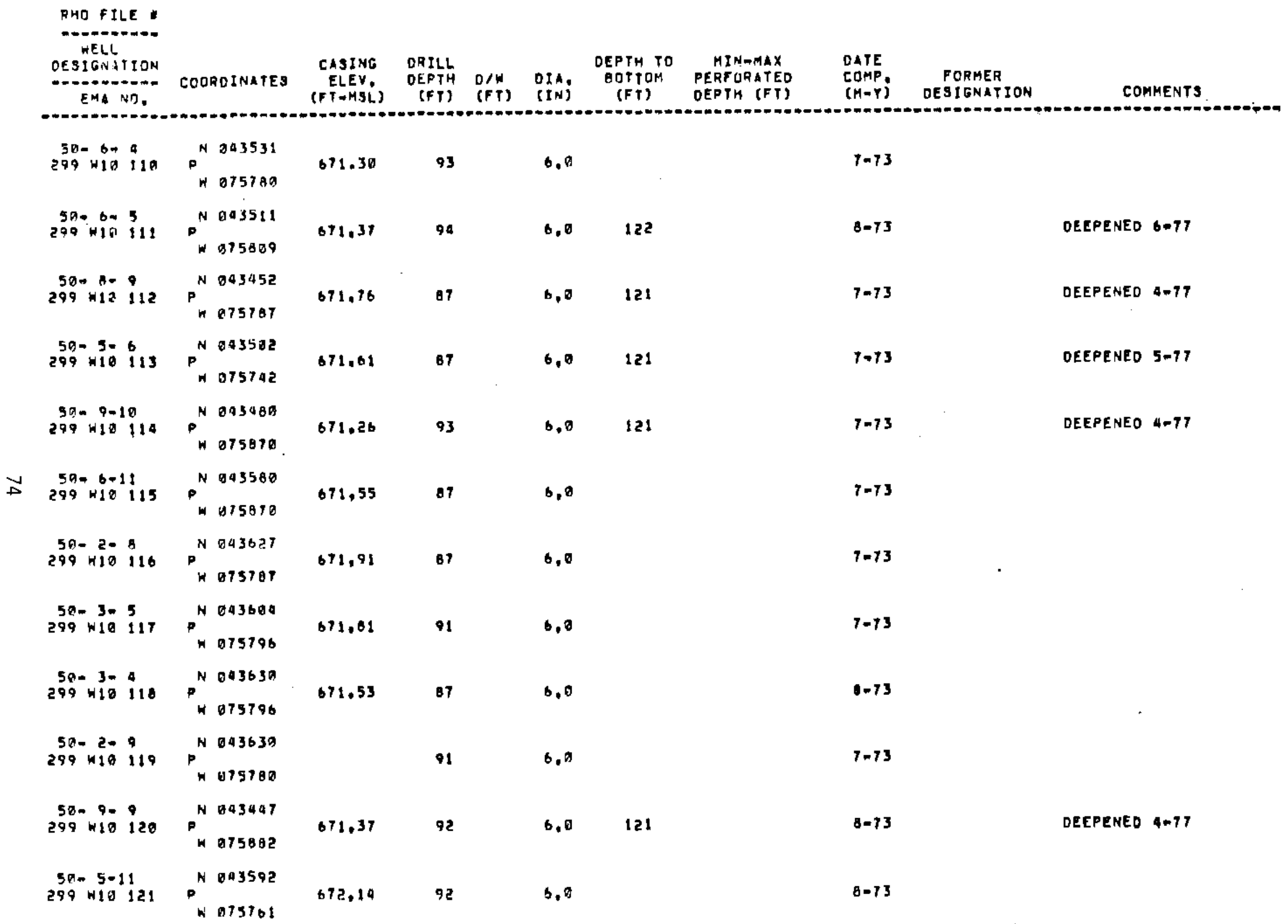




\begin{tabular}{|c|c|c|c|c|c|c|c|c|c|}
\hline $\begin{array}{l}\text { WELL. } \\
\text { DESIGNATION } \\
\text { EMA NO. }\end{array}$ & COOAOINATES & $\begin{array}{c}\text { CASING } \\
\text { ELEY. } \\
\text { (FT=MSL) }\end{array}$ & $\begin{array}{l}\text { ORILL } \\
\text { OEFTH } \\
\text { (FT) }\end{array}$ & $\begin{array}{l}0 / \% \\
(F T)\end{array}$ & OIN\} & $\begin{array}{c}\text { DEPTH TO } \\
\text { BOTTOH } \\
\text { (FT) }\end{array}$ & $\begin{array}{l}\text { MIN-MAX } \\
\text { PERFORATED } \\
\text { OEPTH (FI) }\end{array}$ & $\begin{array}{l}\text { DATE } \\
\text { COMP: } \\
\text { (H-Y) }\end{array}$ & $\begin{array}{l}\text { FORMER } \\
\text { OESIGNATION }\end{array}$ \\
\hline $\begin{array}{rll}50-2= & 2 \\
299 & \text { w19 } & 122\end{array}$ & $\begin{array}{l}P_{N 003680}^{N} 075702 \\
N 040\end{array}$ & 672,51 & 92 & & 0,0 & & & $2-74$ & \\
\hline $\begin{array}{ccc}59-2 \pi & 5 \\
299+10 & 123\end{array}$ & $e^{N 843596}$ & 672,34 & 91 & & 6.8 & & & $3-79$ & \\
\hline $\begin{array}{l}50-2-10 \\
290 w 18120\end{array}$ & $\begin{array}{l}\text { N } \\
\text { H } 04578688\end{array}$ & 671.70 & 91 & & 0.0 & & & $3 \cdot 78$ & \\
\hline $\begin{array}{l}50-2-12 \\
299=10125\end{array}$ & $P^{N 043693}$ & 672,14 & 92 & & 6,0 & & & 2.74 & \\
\hline 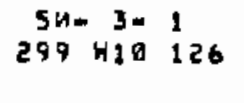 & $\begin{array}{l}P_{N}^{N} 045093 \\
P^{N} 875018\end{array}$ & 691.88 & 92 & & 6.0 & & & $2-74$ & \\
\hline 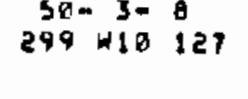 & $\overbrace{}^{N 043634}$ & & 91 & & 6,0 & & & 2.74 & \\
\hline $\begin{array}{c}58-3-10 \\
299 \text { w14 } 128\end{array}$ & $\begin{array}{l}P^{N} 043678 \\
N 075870\end{array}$ & 672,82 & 93 & & 6.0 & & & $2-74$ & \\
\hline $\begin{array}{ccl}50-4-5 \\
299 & =10 & 129\end{array}$ & $P_{H}^{N 015620}$ & 673,73 & 94 & & 6,0 & & & 3.14 & \\
\hline $\begin{array}{c}5 a-4=10 \\
299 w 10130\end{array}$ & $\begin{array}{l}P_{H}^{N} 043567 \\
H 075687\end{array}$ & 672.94 & 93 & & 6,8 & & & $3-74$ & \\
\hline 299410131 & & & & & & & & 0.0 & \\
\hline $299 \omega 10132$ & & & & & & & & $\theta=0$ & \\
\hline $\begin{aligned} 50-8=7 \\
299+10 \\
90\end{aligned}$ & $\begin{array}{l}P^{N} 343397 \\
N 15761\end{array}$ & 671,93 & 94 & & 6,0 & & & $2-74$ & \\
\hline
\end{tabular}


RHU FILE

WELL

DESIGNATION

EMA NOI,

COOROLHATES

CASING DRILL

$\begin{array}{ccccc}\text { DRILL } & & \text { DEPTH TO } & \text { HIN-MAX } \\ \text { DEPTH } & \text { O/W } & \text { DIA } & \text { BOTTOM } & \text { PERFORATEO } \\ \text { (FT) } & \text { (FT) } & \text { (IN) } & \text { (FT) } & \text { DEPTH (FT) }\end{array}$ (FT-MSL)

(FT) (FT)

(F T)

PERFORATEO
DEPTH (FT)

DATE

CUMP. FORMER

(M-Y) DESIGNATION

COMMENTS

$59=9.5 \quad N 043397$

299 110 130

$P$ P

$671.68 \quad 99$

0,0

$3=74$

$50-16-5$

299 w10 135

N 043305

P. 075610

673.84

94

6.0

$2 \times 74$

$50-10 \cdot 7$

$299+10136$

N 043305

$P .075657$

073.20

94

6,0

$2=74$

$50-10-10$

$299 \times 10137$

N 003367

P 075004

672.16

94

6.0

$2=74$

$5 H-11 \% 5$ N 043305

299 मी 138

$P \quad 075717$

672.47

93

6,0

$2=74$

$59-11=8 \quad N 043327$

299 W19 139

H 075796

671.61

94

6.6

$2 m 74$

50-12=7 N 043305 - 075855

671,82

94

6,0

$3+74$

$50-12-10$

N 043365

P 175888

671.63

94

$.6,6$

121

$3-74$

OEEPENEO 2-T7

$50-1+7$

N 043397

299410142

H 075650

$672.95 \quad 90$

6.0

$3 * 74$

$290-B=5$ N10 143043900

299 W10 143

H 875716

672,71

93

6,0

$3=74$

$50-9 \pi 7$

N 043597

$299410 \quad 244$

P 075057

670.92

94

6.0

$3-74$

50. 3. $t$

N 943603

$299 \times 10 \quad 145$

H 075447

$670.00 \quad 99$

$6.6 \quad 123$

$1-75$

DEEPENED $3-77$ 


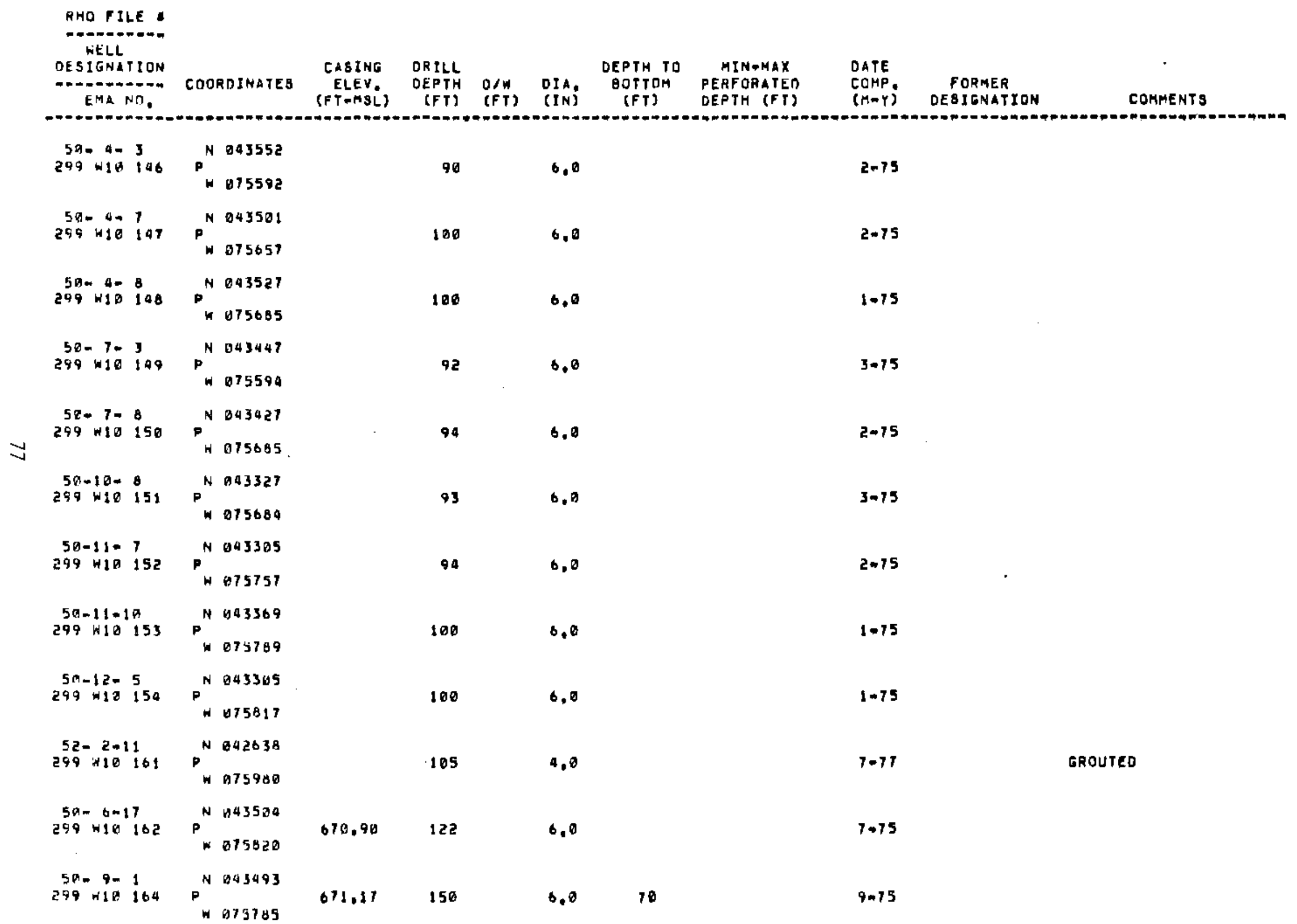




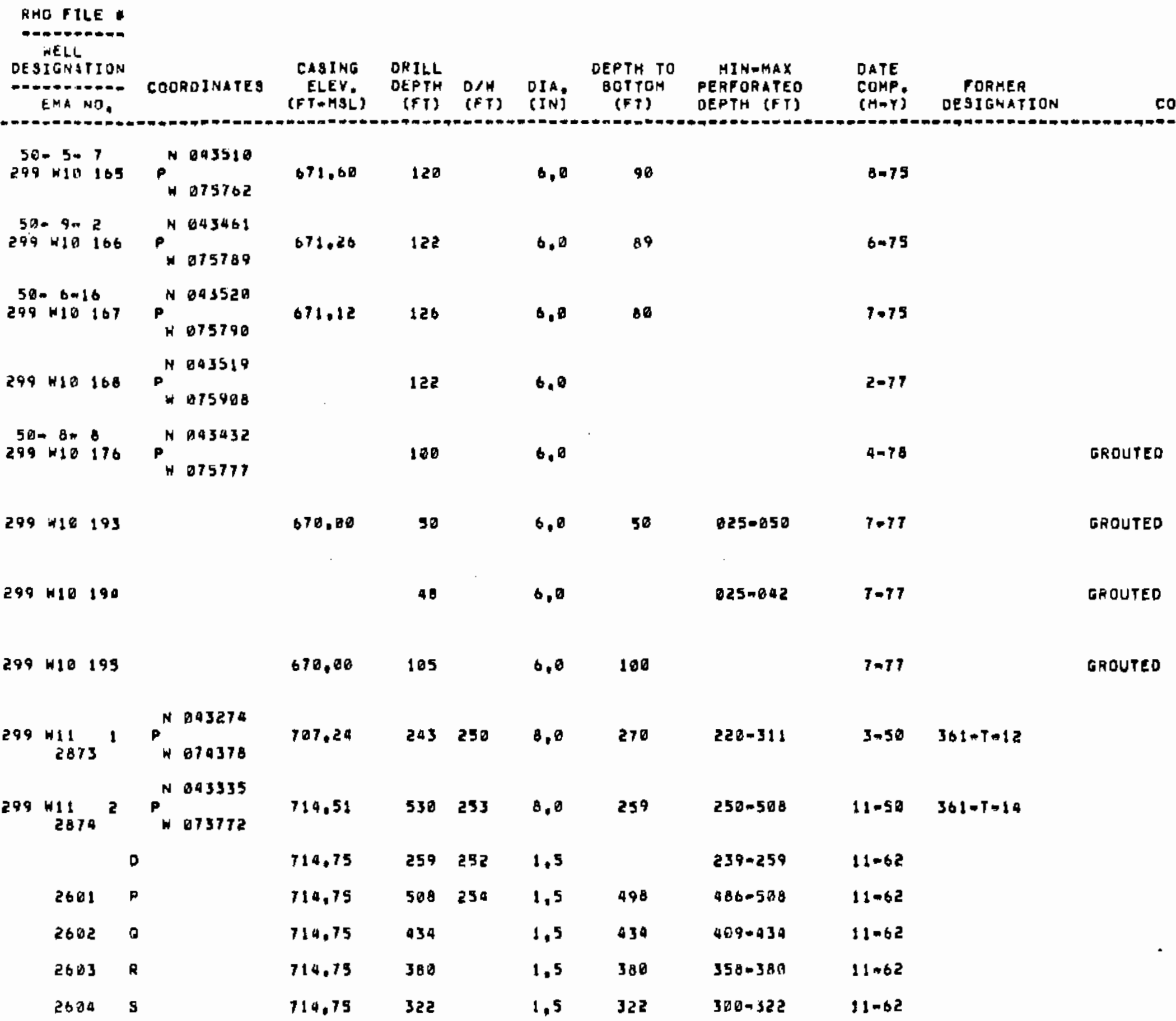




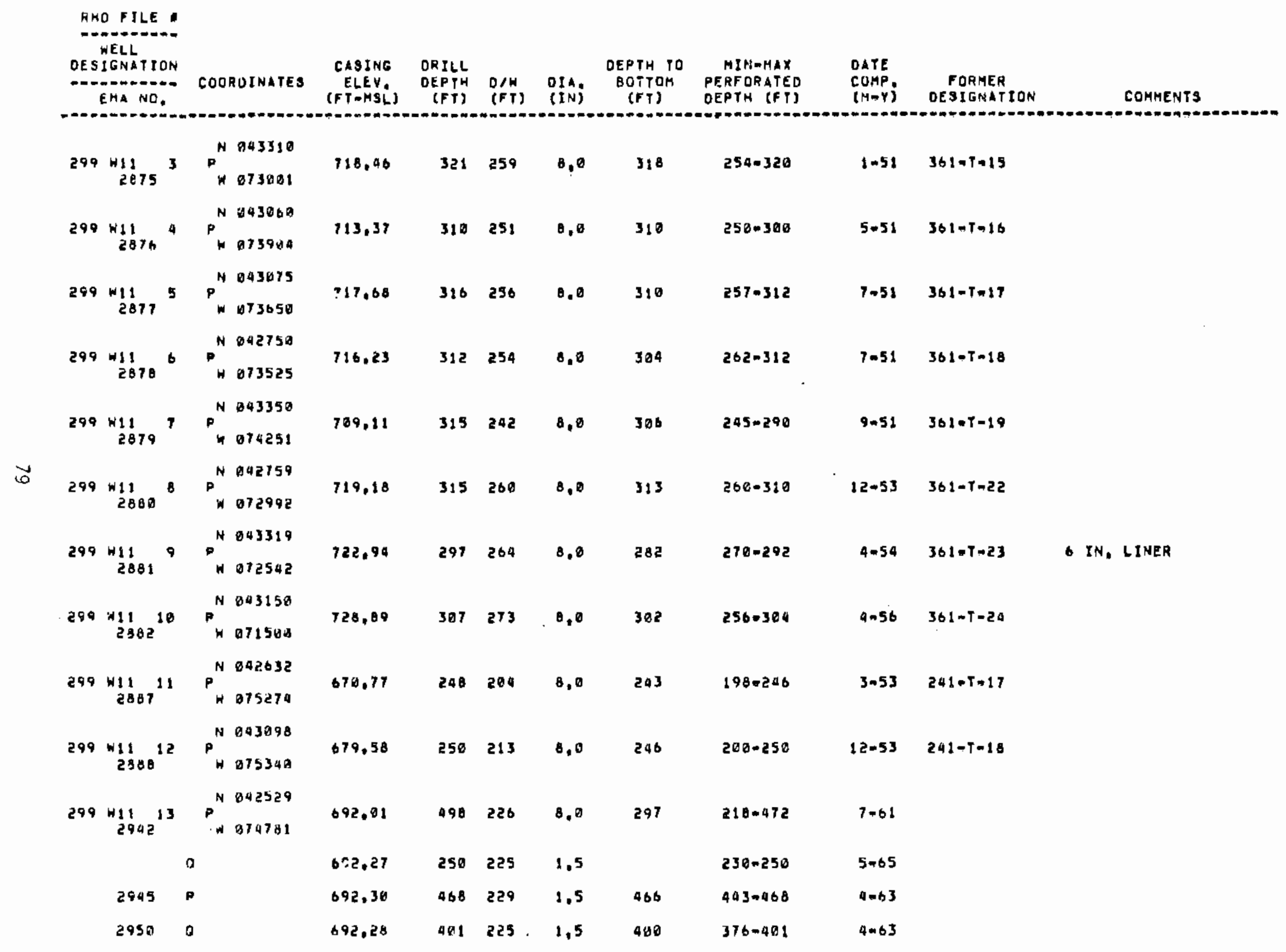


RHO FILE

$$
\text { HELL }
$$

DESIGNATION

DESIGNATION

EML NO.

COORDINATES

CASING

DFTH DIW DIA, BOTTOM PEAFORATEO PEFORATEO DEPTH (FT)

DATE

COMP, FORMER

(M-Y) DESIGNATION

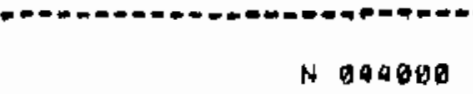

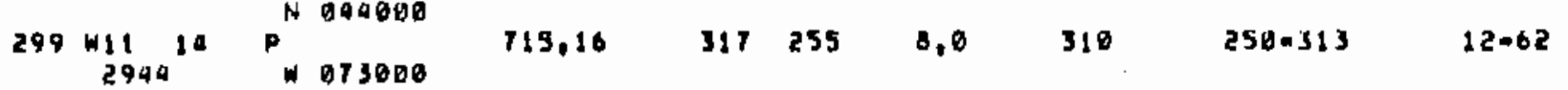

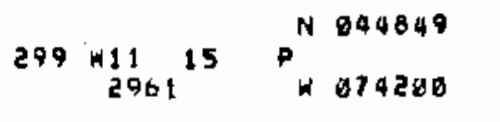

265298

$6,0 \quad 262$

$240-263$

$12=65$

$299+11$ 16 P $P^{N} 044720$

106.00

365246

6.8

363

$343=357$

$82-65$

$299 \mathrm{H1} 17 P^{N 094659}$

$705,00 \quad 300 \quad 293$

248

$2230295 \quad 2-67$

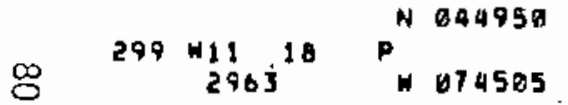

$107.00 \quad 300 \quad 243$

$302 \quad 227 \cdot 295$

$3-67$

$299 H 1119 P^{N 044870}$

707.00

$364 \quad 244$

$6.0 \quad 385$

$234-365$

4969

$299 \times 1120 P^{N 044850}$ 2976 H 074520

$706.00 \quad 266 \quad 293$

$2 b 1$

NDNE

$6-69$

$279+11$ 21 P $P^{N 44850}$

$700.00 \quad 270 \quad 243$

$264 \quad 2350267$

$3-69$

50-0.2 N 243766

290 W11 $23 P_{H} 075416$

$252 \quad 220$

0.0

$200-240$

1073

$299 \times 1124 \mathrm{P}^{\mathrm{N}} 243716$

250

$$
6.0
$$

$200-250$

$8=73$

$299+1126$

890,00

515

3,5

405

NONE

$4=76 \quad \mathrm{OH}=6$

$50-\mathrm{U}-3$

N $\$ 43648$

299 HII 51

P 175577

$677,37 \quad 100$

$0,0 \quad 148 \quad 50-150$

$9=44$

$241-T-131$

$T+133 \quad 10.91$ 


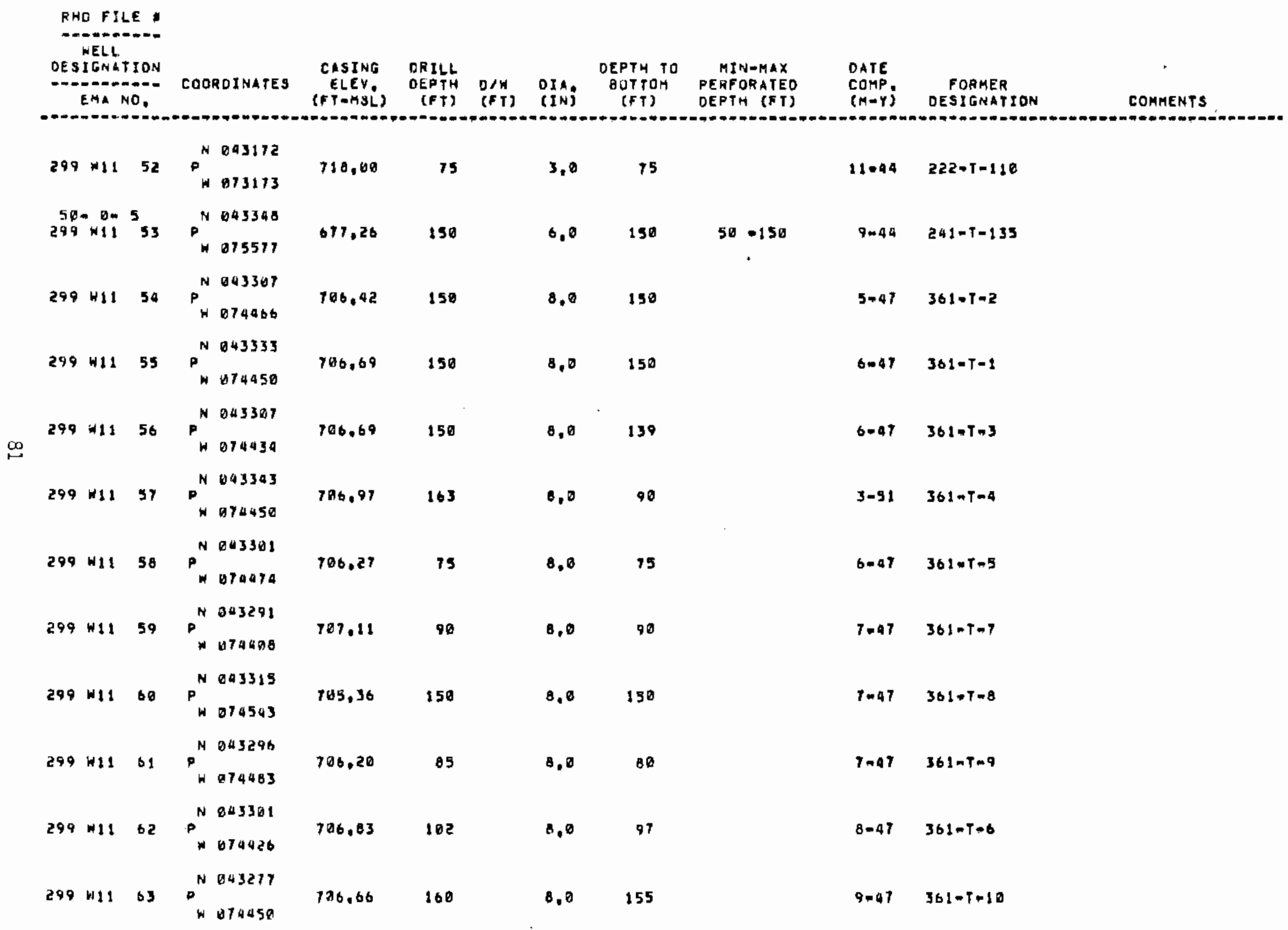




\section{RHO FILE}

OHEDTh

DESIGNATION ema NO.

COORDINATES

CASING DRTLL

DEPTH (F T HSL)

DEPTH TO
DIW DIA, BOTTOM

MINMMAX
PERTORATED PERF DRATED
DEPTH (FT)

DATE

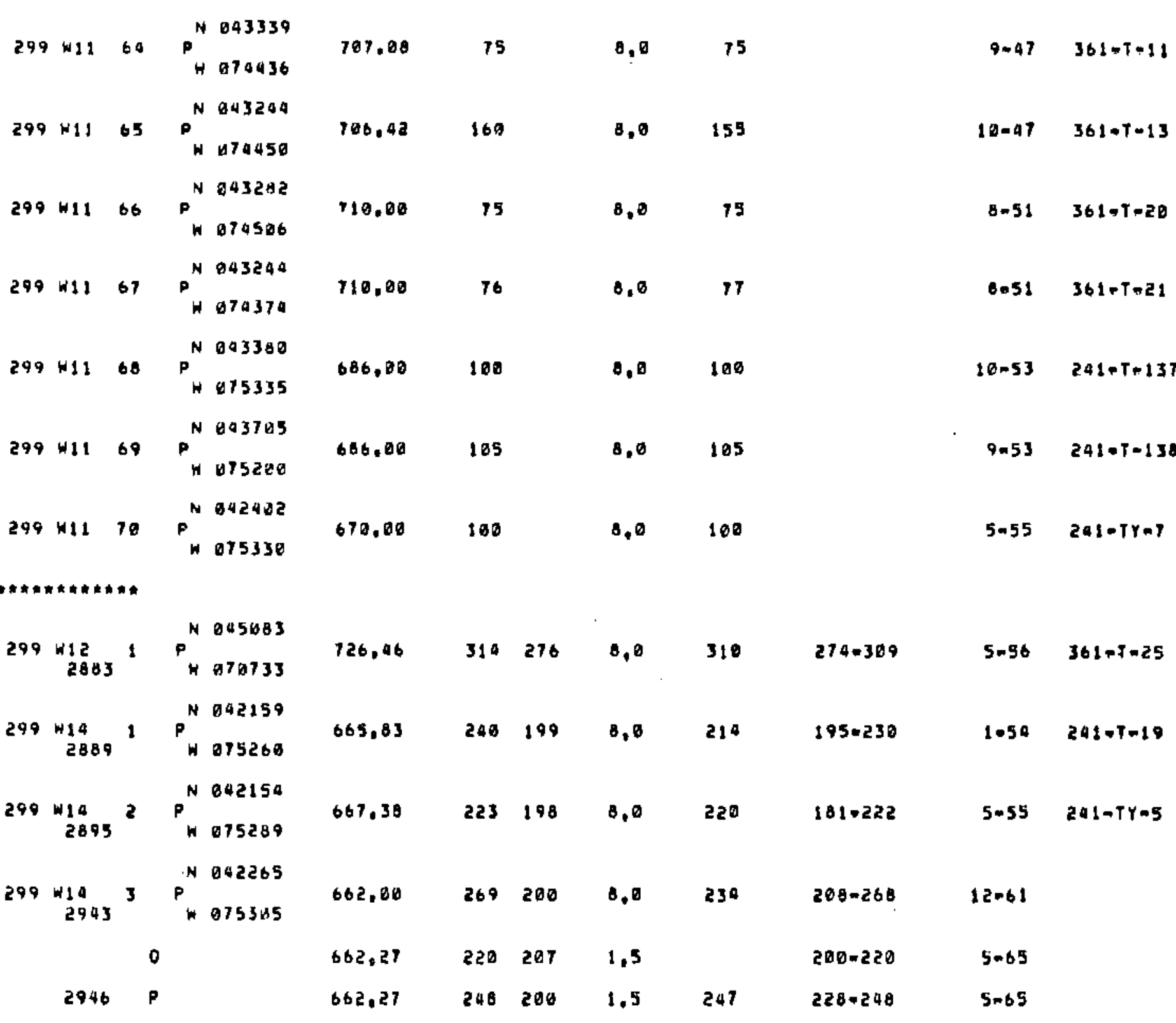

(IN)

( $F T$ )

EPTH (FT)

$(M-Y)$

PORHER DESIGNATION

COMKENTS 


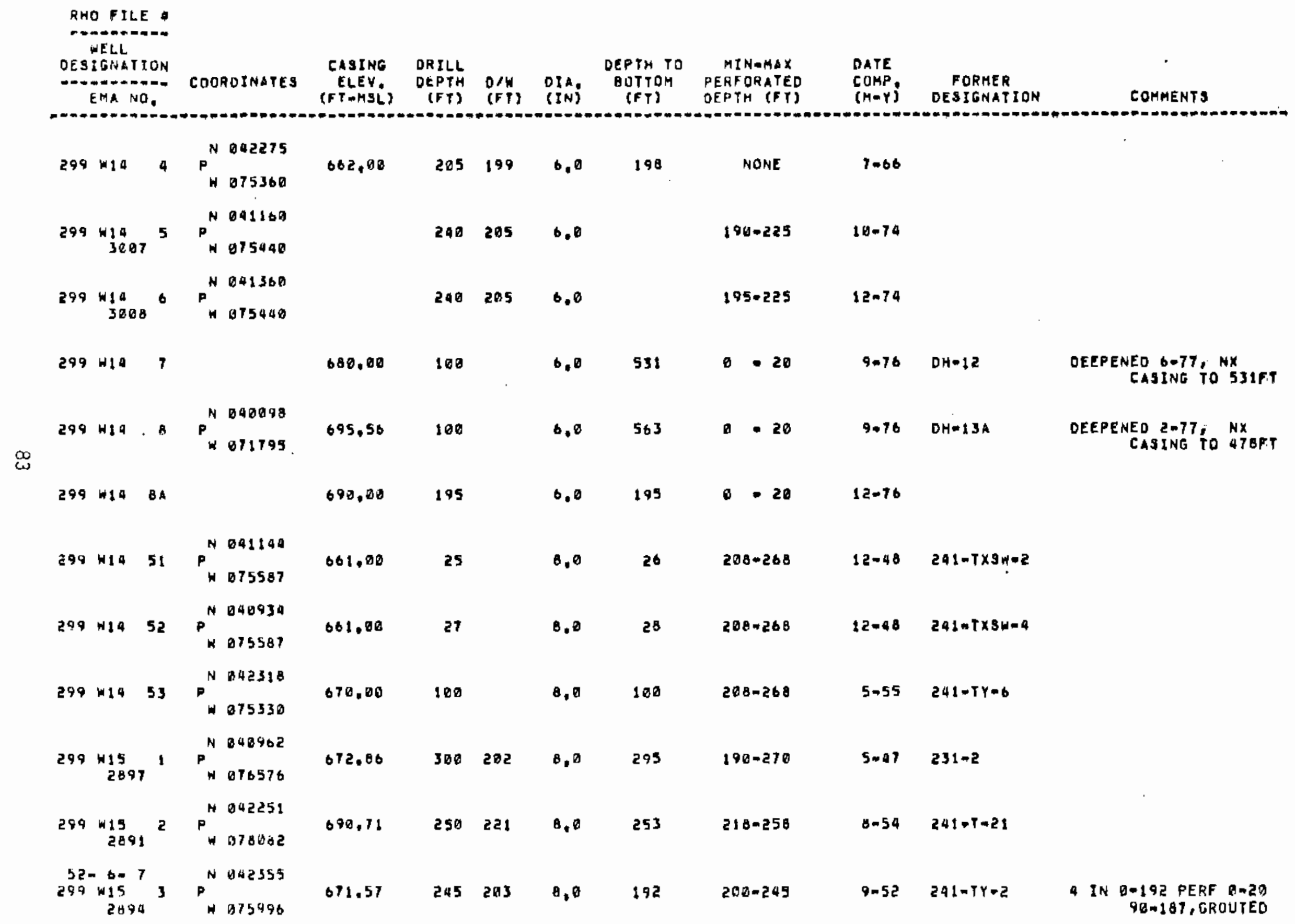




\begin{tabular}{|c|c|c|c|c|c|c|c|c|c|c|c|c|}
\hline ogs: & 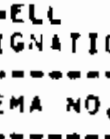 & & CODROINATES & $\begin{array}{l}\text { CASING } \\
\text { ELEV. } \\
\text { (FT=MSL) }\end{array}$ & $\begin{array}{l}\text { DRILL } \\
\text { DEPTH } \\
\text { (FT) }\end{array}$ & (Fr) & $\begin{array}{l}\text { OIAP } \\
\text { GINj }\end{array}$ & $\begin{array}{l}\text { DEPTK TO } \\
\text { BOTTDM } \\
\text { (FT) }\end{array}$ & $\begin{array}{l}\text { HIN-MAX } \\
\text { PERFURATED } \\
\text { OEPTH (FT) }\end{array}$ & $\begin{array}{l}\text { DATE } \\
\text { COMP, } \\
(M \rightarrow Y)\end{array}$ & $\begin{array}{l}\text { FORMER } \\
\text { DESIGNATION }\end{array}$ & COMMENTS \\
\hline 299 & $\begin{array}{l}15 \\
2496\end{array}$ & $a$ & & 662.08 & 217 & 198 & 8,0 & 212 & $170 \cdot 216$ & 1.56 & $241-T x=12$ & \\
\hline 299 & $\begin{array}{l}W 14 \\
2922\end{array}$ & 5 & $P^{P} 075984$ & 678.68 & 599 & 199 & 8,0 & 340 & 173.524 & 4.57 & & \\
\hline & & & 0 & 678,95 & 220 & 194 & 1,5 & & $200-220$ & 5.65 & & \\
\hline & & & $\mathbf{P}$ & 679.95 & 594 & 280 & 1.5 & 418 & 529.994 & $10-64$ & & \\
\hline & & & 0 & 670.95 & 520 & 199 & 1.5 & 336 & $495-520$ & $10-64$ & & \\
\hline & & & $R$ & $67 \%, 95$ & 460 & 199 & 1,5 & 448 & $435-460$ & $10-64$ & & \\
\hline & & & $\mathbf{s}$ & 670,99 & 400 & 199 & 1.5 & 393 & $375=400$ & $10-64$ & & \\
\hline 299 & $\begin{array}{l}W 15 \\
2930\end{array}$ & 6 & $P_{W 075765}$ & 661.50 & 4.0 & 190 & 0.0 & 370 & $275=488$ & $3-59$ & & $\begin{array}{c}\text { SA, PUMP, } 310^{\circ}-8^{\circ} \mathrm{CSN} O \\
100^{\circ}-6^{\circ} \text { CSNE }\end{array}$ \\
\hline 299 & $\begin{array}{l}W 15 \\
2960\end{array}$ & 7 & ${ }^{P}{ }_{W} 076180$ & 664.00 & 350 & 195 & 8.6 & 325 & $182+350$ & $3=66$ & & \\
\hline 299 & 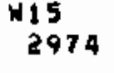 & B & & 667.79 & 206 & 106 & 8.8 & 203 & NONE & $11-66$ & 115:83 & $\begin{array}{c}\text { 6" LINER, DEEPENEOA } \\
\text { SAMPLE PUAP }\end{array}$ \\
\hline 299 & $\begin{array}{l}+15 \\
2975\end{array}$ & 9 & $P_{W}^{N} 075890$ & 662.30 & 195 & 198 & 8,0 & 191 & NONE & $12-66$ & $115=94$ & $\begin{array}{c}\text { 6" LINER, DEEPENED } \\
\text { SAMPLE PUMP }\end{array}$ \\
\hline 299 & $\begin{array}{l}W 15 \\
2699\end{array}$ & 10 & $\begin{array}{l}P^{N} 091080 \\
N 076920\end{array}$ & 076.08 & 300 & 205 & 8,6 & 298 & $183=297$ & $1-68$ & & \\
\hline 299 & $\begin{array}{l}15 \\
2610\end{array}$ & 11 & 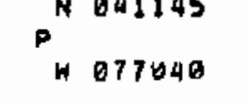 & 677.08 & 300 & 207 & 8,6 & 300 & $183+297$ & $3-68$ & & \\
\hline 299 & W19 & 12 & P. 076095 & 671,00 & 225 & 200 & 6.0 & 229 & 195.219 & $10-73$ & & \\
\hline
\end{tabular}




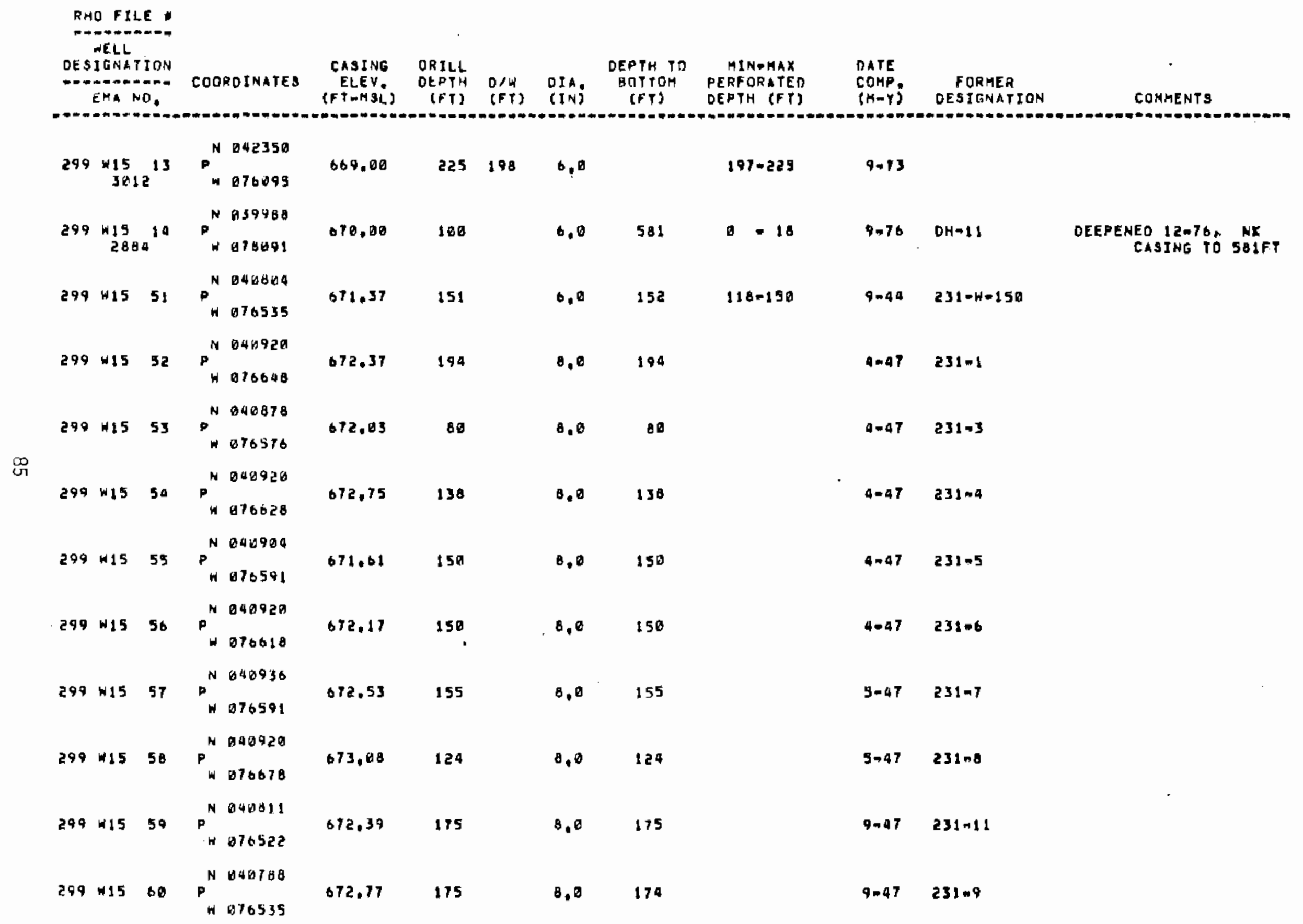




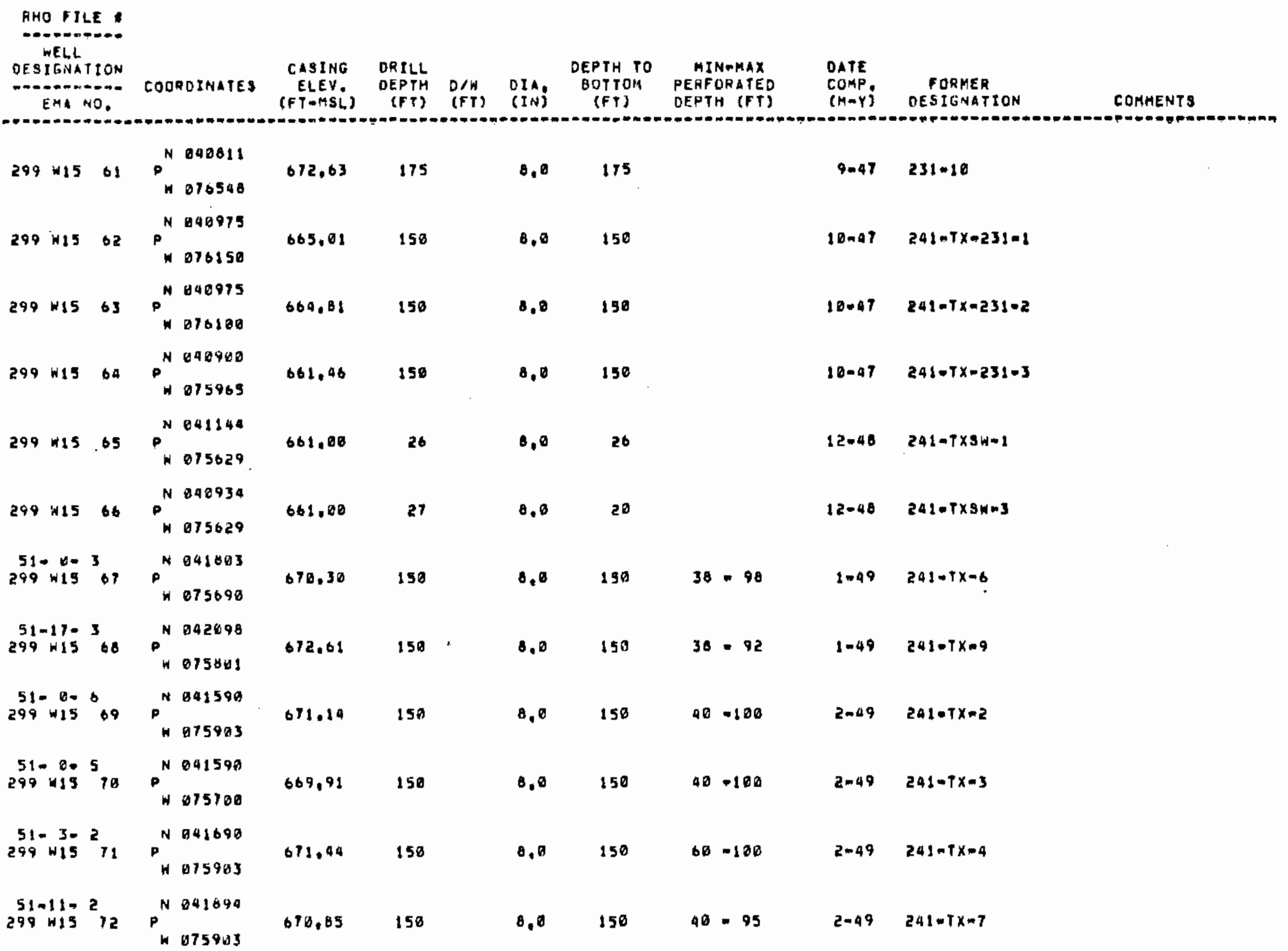




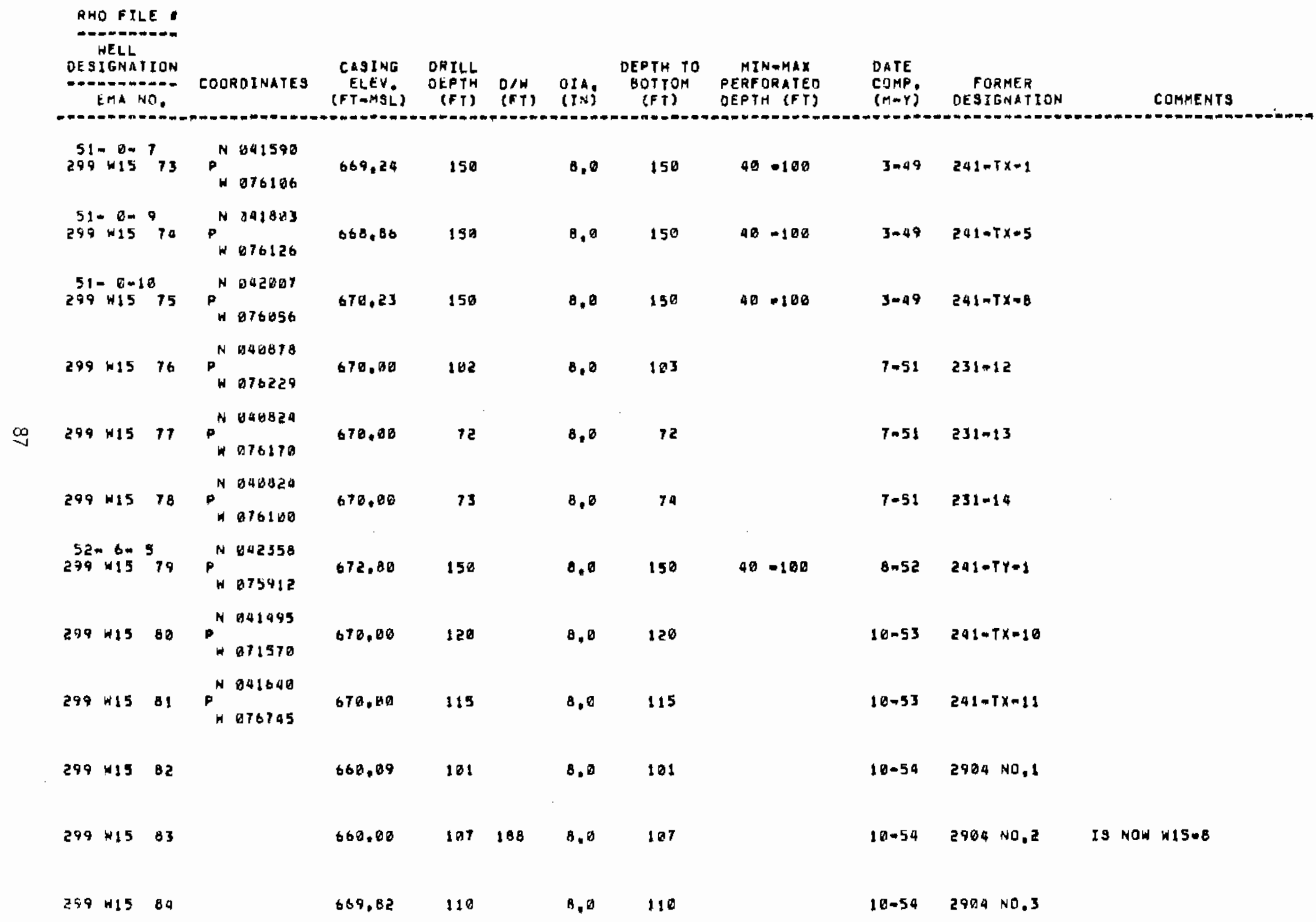


RMO FILE

DELI

DESIGNATION

DEA NO

CODADINATES

ELEV.

TH O/W

DIA DEPTH TO

PERFORATED

DEPTH (FT)

DATE

COMP FDRHER

DESIGNATION

COMHENTS

\begin{tabular}{|c|c|c|c|c|c|c|c|c|c|c|}
\hline 299 & 19 & 65 & & & 664.11 & 106 & 8,0 & 106 & $10 \cdot 54$ & $2904 N 0.4$ \\
\hline 299 & 115 & 66 & $P^{n}$ & $\begin{array}{l}139790 \\
075958\end{array}$ & 661.22 & 149 & 6,0 & 149 & 0.57 & \\
\hline 299 & 115 & 67 & & & 370.00 & 26 & 8.0 & 26 & $12 * 48$ & $241-T X=1 T . H$. \\
\hline 299 & $H \leq 5$ & 86 & & & 670.00 & 26 & 8,0 & 26 & $12-48$ & $241-T x=2 T, H$. \\
\hline 299 & $w 15$ & 09 & & & 670.00 & 27 & 8,0 & 27 & $12 \cap 4 B$ & $241-T X-3 T, H$. \\
\hline 299 & 115 & 90 & & & 670.00 & 20 & & 28 & $12=48$ & 24I-TXUAT,H. \\
\hline 299 & 15 & 91 & & & 670.00 & 150 & & 156 & $10-47$ & $241-T X-13$ \\
\hline 299 & W15 & 92 & & & 679.20 & 150 & . & 150 & 10.47 & $241-T x=14$ \\
\hline 299 & $w 15$ & 93 & & & 670.00 & 150 & & 150 & $10-47$ & $241-T X=15$ \\
\hline 299 & 115 & 94 & $P_{W}^{N}$ & $\begin{array}{l}039930 \\
075890\end{array}$ & 600,00 & 100 & 0.0 & 100 & $\ln 59$ & \\
\hline 299 & W15 & 95 & $P^{N}$ & $\begin{array}{l}039939 \\
675925\end{array}$ & 660,00 & 100 & 8,0 & 100 & 1059 & \\
\hline 299 & w15 & 96 & $P_{H}^{N}$ & $\begin{array}{l}942446 \\
975933\end{array}$ & 672,60 & 70 & 6.0 & 70 & $|-6|$ & \\
\hline
\end{tabular}

I8 NOW W15e9 


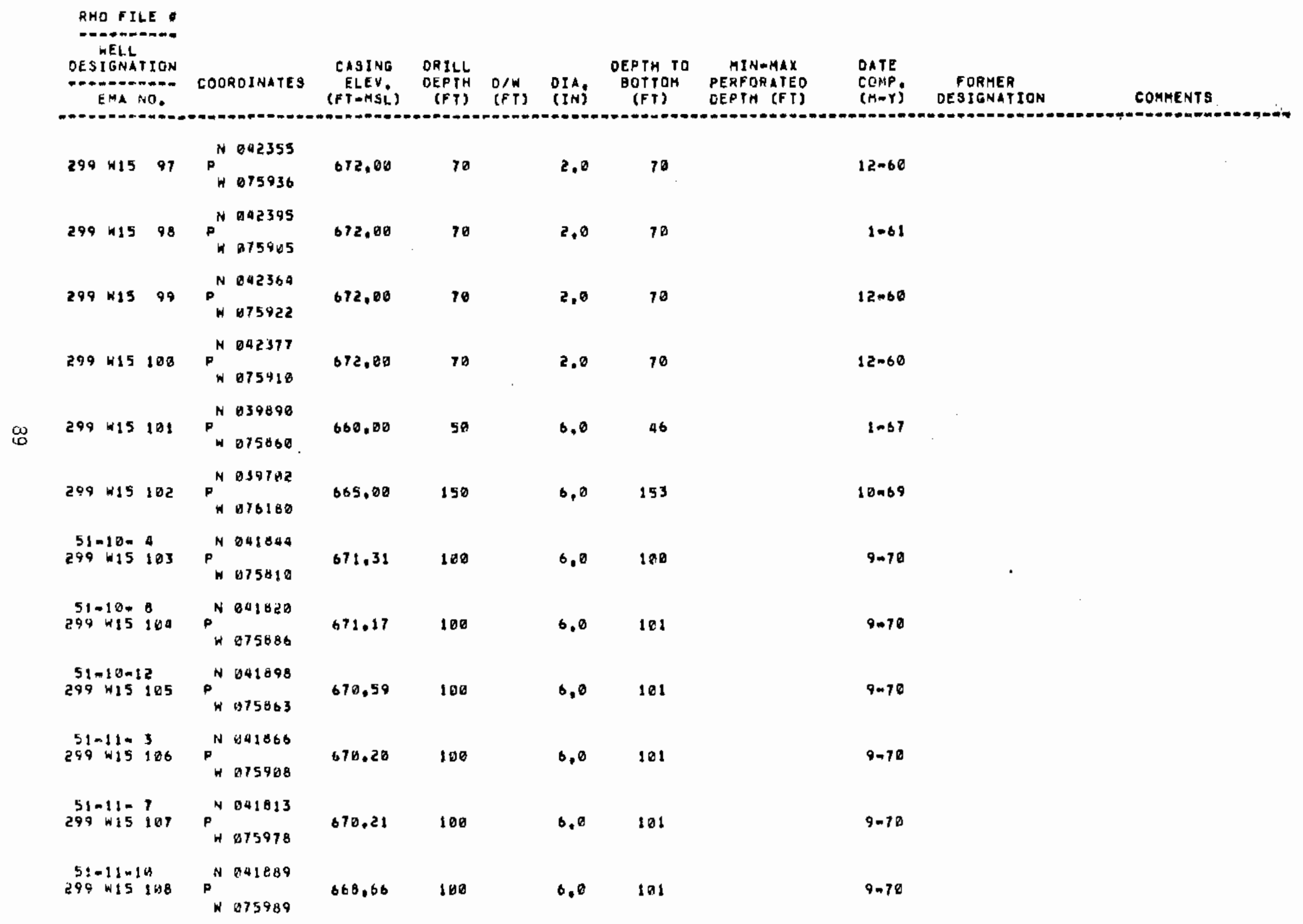


DESIGNATION

EMA NO

COOROINATES

CASING DRILL

DEPTH TO

MINAMAX

PERFORATED

DATE

(FTMMSL) (FT) (FT) (IN) (FT) OEPTH (FT)

COMP FORMER

$(\mathrm{CH}-\mathrm{Y})$

DSIGNATION

COMMENTS

\begin{tabular}{|c|c|c|c|c|c|c|c|c|}
\hline $\begin{array}{r}51-12= \\
299 \times 15\end{array}$ & 489 & $P^{N}$ & $\begin{array}{l}041842 \\
076310\end{array}$ & 669,35 & 100 & 6,0 & 101 & 9.70 \\
\hline $\begin{array}{r}51-12- \\
299+15\end{array}$ & 7 & $P^{H}$ & $\begin{array}{l}041813 \\
076980\end{array}$ & 668,60 & 100 & 6.0 & 101 & $10-70$ \\
\hline $\begin{array}{r}51 * 12-1 \\
299 \times 15\end{array}$ & 111 & $P_{W}^{N}$ & $\begin{array}{l}041900 \\
076000\end{array}$ & 667.95 & 100 & 6,0 & 102 & 9.70 \\
\hline $\begin{array}{r}51-135 \\
299+15\end{array}$ & $\begin{array}{l}5 \\
112\end{array}$ & $P^{N}$ & $\begin{array}{l}841922 \\
075116\end{array}$ & 670.12 & 100 & 6,0 & 101 & $10-70$ \\
\hline $\begin{array}{r}51=13= \\
289+15\end{array}$ & 8 & $e^{N}$ & $\begin{array}{l}041929 \\
075781\end{array}$ & 671.86 & 100 & 6.0 & 101 & $9 \cdot 78$ \\
\hline $\begin{array}{r}51-13-1 \\
299 \text { W15 }\end{array}$ & 12 & $P_{W}^{N}$ & $\begin{array}{l}042090 \\
075750\end{array}$ & 671.19 & 100 & 6.5 & 101 & $10-70$ \\
\hline $\begin{array}{r}51-14= \\
299 \times 15\end{array}$ & 115 & $P^{N}$ & $\begin{array}{l}041950 \\
075859\end{array}$ & 670.75 & 100 & 6.0 & 101 & 9.70 \\
\hline $\begin{array}{r}51-145 \\
299+15\end{array}$ & 9 & $P_{H}^{N}$ & $\begin{array}{l}191922 \\
675886\end{array}$ & 669,90 & 100 & 6.0 & $10 !$ & 9.78 \\
\hline $\begin{array}{r}51-10-1 \\
299 \times 15\end{array}$ & 117 & $D^{N}$ & $\begin{array}{l}041999 \\
975863\end{array}$ & 669.94 & $10 n$ & 6,8 & 101 & 9.70 \\
\hline $\begin{array}{r}51-15= \\
299+15\end{array}$ & 118 & $P^{N}$ & $\begin{array}{l}041949 \\
075912\end{array}$ & 369,60 & 100 & 0,0 & 101 & $3 \cdot 70$ \\
\hline $\begin{array}{r}51-15= \\
299 \times 15\end{array}$ & 719 & $P_{H}^{N}$ & $\begin{array}{l}041923 \\
075482\end{array}$ & 668,65 & 100 & 6,0 & 101 & $3-70$ \\
\hline $\begin{array}{l}51-15-1 \\
299 \mathrm{WI}\end{array}$ & 120 & $P^{N}$ & $\begin{array}{l}049496 \\
075969\end{array}$ & 689,23 & 100 & 0,0 & & $0=0$ \\
\hline
\end{tabular}




\begin{tabular}{|c|c|c|c|c|c|c|c|c|c|}
\hline $\begin{array}{c}\text { HELL } \\
\text { OESIGNATION } \\
\text { EMANO }\end{array}$ & COOROINATES & $\begin{array}{l}\text { CASING } \\
\text { ELEV } \\
\text { (FT }=\text { MSL })\end{array}$ & $\begin{array}{l}\text { DRILL } \\
\text { DEPTH } \\
\text { (FT) }\end{array}$ & $\begin{array}{l}0 / H \\
(F T)\end{array}$ & DIAj & $\begin{array}{l}\text { DEPTH TO } \\
\text { BOTTOM } \\
\text { (FT) }\end{array}$ & $\begin{array}{l}\text { MINAGAX } \\
\text { PERFORATEO } \\
\text { DEPTH (FY) }\end{array}$ & $\begin{array}{l}\text { QATE } \\
\text { COMP, } \\
\text { (MTY) }\end{array}$ & $\begin{array}{l}\text { FORHER } \\
\text { DESIGNATION COMMENTS }\end{array}$ \\
\hline $\begin{aligned} 51-17=2 \\
299 \times 15 \text { 121 }\end{aligned}$ & $\begin{array}{l}P^{N} 042890 \\
N 075818\end{array}$ & 671.23 & 100 & & 6.6 & 101 & & $10-10$ & \\
\hline $\begin{array}{c}51-17-10 \\
299+15122\end{array}$ & $P^{N} 042082$ & 669,92 & 100 & & 6.0 & 101 & & $10-70$ & \\
\hline $\begin{array}{r}51-18=3 \\
298+15123\end{array}$ & $P_{W 045908}^{N}$ & 669.88 & 108 & & 0,0 & 101 & & 10.70 & \\
\hline $\begin{array}{rl}51-187 & 7 \\
249 W 15 & 124\end{array}$ & $P^{N} 01542020$ & 668,77 & 100 & & 6.0 & 101 & & $9 \times 10$ & \\
\hline $\begin{array}{l}51-18=10 \\
299 W 15125\end{array}$ & $\begin{array}{l}P^{N} 042093 \\
N 075909\end{array}$ & 668,75 & 100 & & 6.0 & 101 & & $18=70$ & \\
\hline $\begin{array}{c}51-3-12 \\
299+15126\end{array}$ & $P_{W 075954}^{N 041098}$ & 671.03 & 100 & & 0,0 & 100 & & $11 \cdot 71$ & \\
\hline 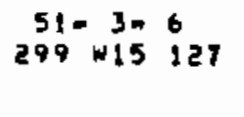 & $\begin{array}{l}P_{N}^{N} 0159042 \\
N 01594\end{array}$ & 670.83 & 100 & & 6,0 & 100 & & $11-71$ & \\
\hline $\begin{array}{r}51-3=9 \\
299+15 \\
128\end{array}$ & $p^{N} 041654$ & 669,34 & 100 & & 0.0 & 100 & & $11-71$ & \\
\hline $\begin{array}{c}51 \% 4-12 \\
299 w 15129\end{array}$ & $\begin{array}{l}P^{N} 441698 \\
N 10056\end{array}$ & 670.69 & 100 & & 6.0 & 100 & & 11.71 & \\
\hline $\begin{array}{rl}51-4+ & 5 \\
299 * 15 & 138\end{array}$ & $\begin{array}{l}P^{N} 091616 \\
N 076022\end{array}$ & 670.69 & 100 & & 6,0 & 100 & & $11+71$ & \\
\hline 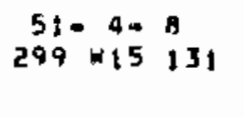 & $\begin{array}{l}P_{H}^{N} 841626 \\
H \quad 016096\end{array}$ & 668,57 & 100 & & 6.0 & 100 & & $11 \cdot 71$ & \\
\hline $\begin{array}{c}51-7-11 \\
299 \times 15132\end{array}$ & $\begin{array}{l}P^{N} 041794 \\
H 075978\end{array}$ & 670,08 & 100 & & 6.0 & 106 & & $11-71$ & \\
\hline
\end{tabular}


$\rightarrow E L L$

DESIGNITION

EMA NO.

CODROINATES

CASING DHILL

ELEY, DEPTH OJH OIA BOTTOH PERFORATED (FT-MSL) (FT) (FT) (IN) (FT) DEPTH (FT)

DATE

COMP. FORMEA

(M-Y) OESIGNATION

COMMENTS

$51-7 n a \quad N 641721$

$\begin{array}{rl}51-7 * a & N 041721 \\ 299+15133 & P \\ & \end{array}$

6.0100

12-71

51\% In 9 N 891760

299 W15 13a P 276066

669.81

6.0100

$11=71$

$51-8.11 \quad N 041790$

299 his 135 P

$668.59 \quad 100$

$6.8 \quad 100$

$11=71$

$31=8.5 \quad N 041718$

$299+15136$ P 407622

$070,04 \quad 100$

6.0200

$11-71$

$5100=9 \quad N$ Dat740

299 W15 $137 \quad P$ N16104

$600.02 \quad 100$

6,0100

$11-71$

$51=9.12$

299 W15 138 P $\quad$ W075759

N 041902

$670,78 \quad 100$

6.0100

$18-71$

$51.9-3 \quad N 041870$

$299+15139$

P 075709

$669,77 \quad 100$

$6,6 \quad 100$

$10-71$

$51-9.8$

N 841420

671.85

$6.0 \quad 100$

10071

$51=2-12 \quad N 041696$

$299+15141 P^{N} \quad 75852$

$672,22 \quad 100$

6,018

$7=71$

$51+2=9$

N 001046

$299 W 15 \quad 142$

H 075896

671.38

$6,0 \quad 101$

$7-71$

$53=2 \div 5$

N 041617

$299 \times 15143$

w 13519

$672,24 \quad 100$

$6.0 \quad 101$

$7=71$

$51-5-10$

$290 \times 15140$

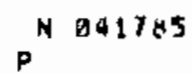

P 675703

673.09

100

0,0101

$6-71$ 


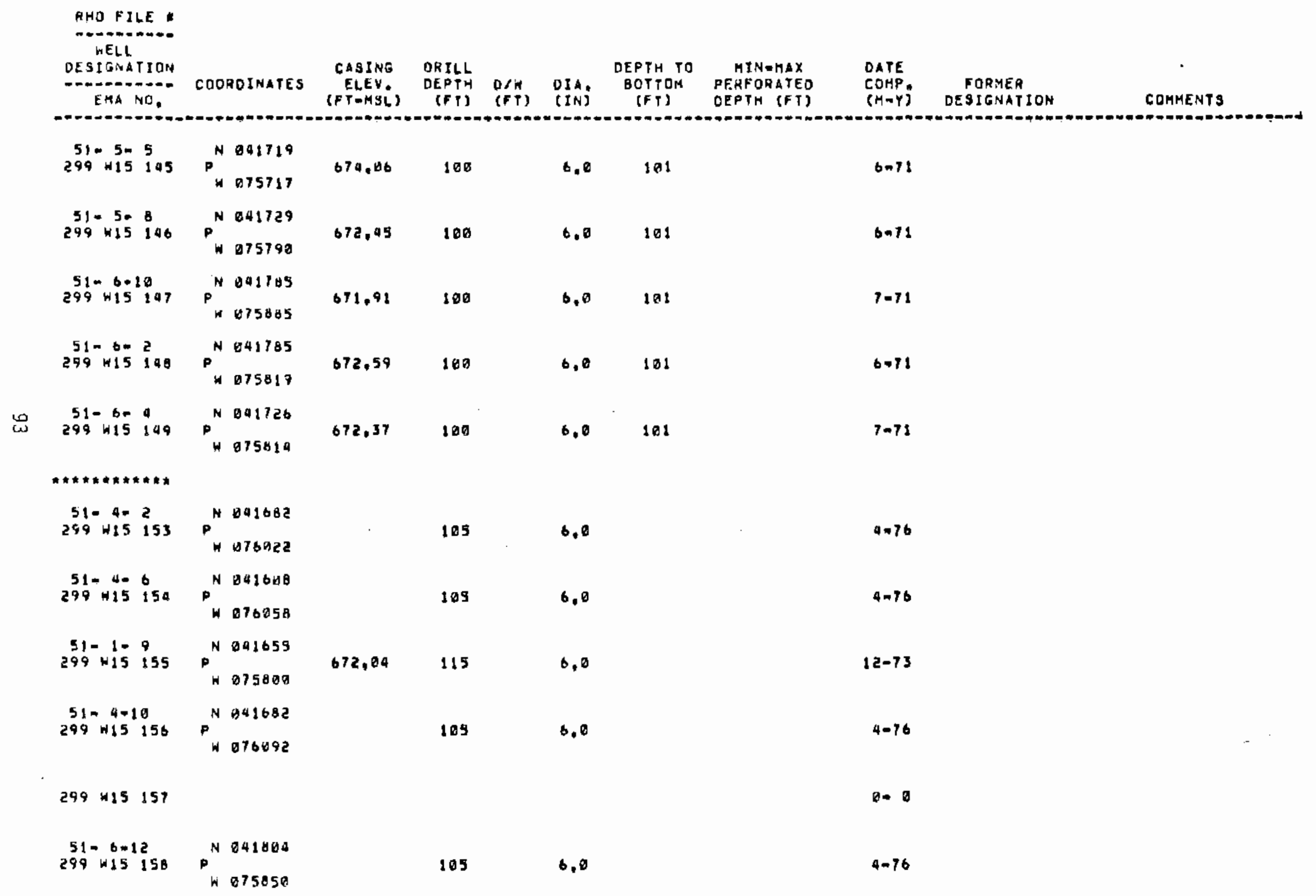


RHO FILE

-

NELL.

OESIGNATION

EHA NO.

COOROINATES

CASING

DRILL

ELEV. DEPTH O/W OIA. BOTTOM PERFORATED

DATE

DOMP, FORER

DESIGNATION

COMMENTS

\section{$51-12=9$ \\ 51-12:1 N 041899}

$299+15159$

P 076030

$660.45 \quad 108$

6,0190

$11=73$

$51-15 * 9$

(4) 041860

$299+15100$

P 875999

$668.80 \quad 100$

6.0100

$12-73$

$51-16=a$

$p^{N} 042348$

069.18

100

$6,0 \quad 108$

$11=73$

$51-16-11, \quad N 842106$

$670,33 \quad 100$

6.0100

11.13

51-16, 7

1. 475765

(1)

$\underset{\infty}{\infty}$

$299+15163$

* 642021

$671.08 \quad 100$

6.0100

$12-73$

$51+17+11 \quad M \quad 642106$

$299+15164 \quad P$ H

$678.46 \quad 100$

6.0100

10.73

$299+15165 \quad P^{N 042186}$

$668.68 \quad 100$

6.0100

$11=73$

$51+1=2$

H 075969

(2)

8,0

$1-74$

51.1. $4 \quad N 041638$

$299+15167$

P. 015708

$672,32 \quad 115$

6,0

$12+73$

$51-1=0 \quad N 641603$

299 W15 168 N075750

$672,03 \quad 100$

6,0

$4 \cdot 74$

299-115 169 P

P 015780

$672.12 \quad 100$

0,0

$4 m 74$

51-2\% 2

N041088

P. $\$ 5620$

$671,93 \quad 100$

6.6

$4=74$ 


\begin{tabular}{|c|c|c|c|c|c|c|c|c|c|}
\hline $\begin{array}{l}\text { WELL } \\
\text { DESIGNATION } \\
\text { EMA NO }\end{array}$ & CODROINATES & $\begin{array}{l}\text { CASING } \\
\text { ELEV, } \\
\text { (FTHMSL) }\end{array}$ & $\begin{array}{l}\text { ORTLL } \\
\text { DEPTH } \\
\text { (FT) }\end{array}$ & $\begin{array}{l}D / W \\
(F T)\end{array}$ & DIA: & $\begin{array}{l}\text { DEPTH TO } \\
\text { BOTTOH } \\
\text { (FT) }\end{array}$ & $\begin{array}{l}\text { MIN-HAX } \\
\text { PERFORATED } \\
\text { DEPYH (FT) }\end{array}$ & $\begin{array}{l}\text { DATE } \\
\text { COMP, } \\
\text { (M=Y) }\end{array}$ & $\begin{array}{l}\text { FORMER } \\
\text { DESIGNATION }\end{array}$ \\
\hline $\begin{array}{rll}51-5 * & 7 \\
290 & \text { W15 } & 171\end{array}$ & $\begin{array}{l}P_{N 041708}^{N} 045763 \\
N 00\end{array}$ & 672,40 & 111 & & 6.0 & 111 & & 1.74 & \\
\hline $\begin{array}{c}51 m 2-7 \\
290 \text { wis } 172\end{array}$ & $\begin{array}{l}P^{N} 041083 \\
N 075863\end{array}$ & 671,53 & 100 & & 0.0 & & & $4=74$ & \\
\hline $\begin{aligned} 5105-5-1 \\
299 \text { wis } 173\end{aligned}$ & $\begin{array}{l}P^{N} 041789 \\
W 075124\end{array}$ & 673.17 & 100 & & 6.8 & & & $3-74$ & \\
\hline $\begin{array}{rl}59-5+3 \\
299 W 15 & 174\end{array}$ & $\begin{array}{l}D^{N} 041757 \\
N 075702\end{array}$ & 679.02 & 115 & & 6.0 & 115 & & 1.74 & \\
\hline $\begin{array}{rll}51 & -6-8 \\
299 & -15 & 175\end{array}$ & $\begin{array}{l}P^{N} 041730 \\
H 015892 .\end{array}$ & 673.77 & 100 & & 0.0 & & & $4-74$ & \\
\hline $\begin{array}{rl}51-10-1 & 1 \\
290 \times 15176\end{array}$ & $\begin{array}{l}P^{N} 041089 \\
N 075019\end{array}$ & 671.93 & 100 & & 6.0 & & & $4=74$ & \\
\hline $\begin{array}{c}51-110 \\
289+15 \text { 17 }\end{array}$ & $\begin{array}{l}P^{N} 041898 \\
H 075959\end{array}$ & 669,67 & 113 & & $b, 0$ & & & $4-74$ & - \\
\hline $\begin{array}{c}51-7 * 1 \\
299 \times 15 \quad 178\end{array}$ & $\begin{array}{l}P^{N} 041799 \\
* 075432\end{array}$ & 670,79 & 110 & & 6.0 & 110 & & $1-74$ & \\
\hline $\begin{array}{rl}51-12= & 5 \\
299 & W 19179\end{array}$ & $\begin{array}{l}P^{N 041808} \\
H 076436\end{array}$ & 669.78 & 100 & & 6.0 & & & $4-74$ & \\
\hline $\begin{array}{l}51-12-18 \\
299^{2} 15180\end{array}$ & $\begin{array}{l}P^{N} 041868 \\
N 076498\end{array}$ & 667.60 & 100 & & 6,8 & & & $4-74$ & \\
\hline $\begin{array}{c}51-18 * 1 \\
299 \text { W15 } 181\end{array}$ & $\begin{array}{l}0^{N} 042103 \\
N 075934\end{array}$ & 689.14 & Ino & & 6.8 & & & $4-74$ & \\
\hline $\begin{array}{c}51-18= \\
299+15182\end{array}$ & $\begin{array}{l}P^{N} 042624 \\
N 075922\end{array}$ & 059.84 & 100 & & 6.0 & & & $9-74$ & \\
\hline
\end{tabular}




\begin{tabular}{|c|c|c|c|c|c|c|c|c|c|c|}
\hline $\begin{array}{l}\text { WELL } \\
\text { DESIGASTION } \\
\text { EMA NO, }\end{array}$ & COORDINATES & $\begin{array}{c}\text { CASING } \\
\text { ELEV. } \\
\text { (FT-HSL) }\end{array}$ & $\begin{array}{l}\text { DRILL } \\
\text { OEFTH } \\
\text { (FT) }\end{array}$ & D/W & DIN; & $\begin{array}{l}\text { DEPTH TD } \\
\text { BOTFOM } \\
\text { (FT) }\end{array}$ & $\begin{array}{l}\text { MINARAX } \\
\text { PERFORATEO } \\
\text { DEFTH (FT) }\end{array}$ & $\begin{array}{l}\text { DATE } \\
\text { COMPP, } \\
\text { (M-Y) }\end{array}$ & $\begin{array}{l}\text { FORMER } \\
\text { DESIGNATIDN }\end{array}$ & COMMENTS \\
\hline $\begin{array}{c}51-18=9 \\
299 \text { W15 } 183\end{array}$ & $\begin{array}{l}P_{1}^{N} 042059 \\
1076001\end{array}$ & 668,24 & 100 & & 6.0 & & & D. 0 & & \\
\hline 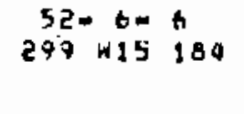 & $\begin{array}{l}P^{N} 042357 \\
W 075938\end{array}$ & 670,81 & 100 & & $B .0$ & & & $8-79$ & & \\
\hline $\begin{array}{rl}52-6 * & 9 \\
299 \times 15 & 185\end{array}$ & $\begin{array}{l}P_{N}^{N} 042385 \\
N 075910\end{array}$ & 670.65 & 100 & & 6.6 & & & $8-74$ & & \\
\hline $\begin{array}{r}52-5 * 7 \\
289 \times 15186\end{array}$ & $\begin{array}{l}P_{W}^{N} 042357 \\
N 45872\end{array}$ & 671,05 & 100 & & 6.0 & & & $8-79$ & & \\
\hline $\begin{array}{rll}51-7 * & 3 \\
299 & 15 & 187\end{array}$ & $\begin{array}{l}P^{N} 041768 \\
H \text { O15903 }\end{array}$ & 670.08 & & & & & & $0=0$ & & \\
\hline $\begin{array}{rl}51 \times 9-4 \\
299 \times 15 & 188\end{array}$ & $\begin{array}{l}P^{N} 041830 \\
W 075714\end{array}$ & 670,00 & 105 & & 6,0 & & & $4 * 76$ & & \\
\hline $\begin{array}{c}51-9-10 \\
299 \times 15180\end{array}$ & $\begin{array}{l}P^{N} 041871 \\
W 075793\end{array}$ & 670,80 & 105 & & 6,8 & & & $4-76$ & & \\
\hline $\begin{array}{rll}51 * 7 * 7 & 7 \\
289 & 15 & 19 p\end{array}$ & $\begin{array}{l}P_{H}^{N} 041714 \\
H 015975\end{array}$ & 670.00 & 105 & & 6,0 & 100 & & $5=77$ & & GROUTED \\
\hline $\begin{array}{r}51-3-11 \\
289 \times 15191\end{array}$ & $\begin{array}{l}P_{N}^{N} 041692 \\
N 5965\end{array}$ & 679,00 & 105 & & 0,0 & 100 & & $5-77$ & & GROUTED \\
\hline $\begin{array}{rll}51-3-1 & 1 \\
289 & W 15 & 192\end{array}$ & $\begin{array}{l}P_{W}^{N} 841092 \\
W 075945\end{array}$ & 670.88 & 105 & & 6,0 & 100 & & 0.77 & & GROUTED \\
\hline $\begin{array}{r}51 \cdot 7.6 \\
299+15193\end{array}$ & $\begin{array}{l}P_{n}^{N} 0459954 \\
n^{N} 07598\end{array}$ & 670,00 & 59 & & 6.8 & 50 & $25 \cdot 50$ & 7.77 & & GROUTED \\
\hline $299 \quad 119194$ & $P_{H} 075958$ & 670.00 & 48 & & 6.0 & & $25 \cdot 4 \theta$ & 7.71 & & GROUTEO \\
\hline
\end{tabular}




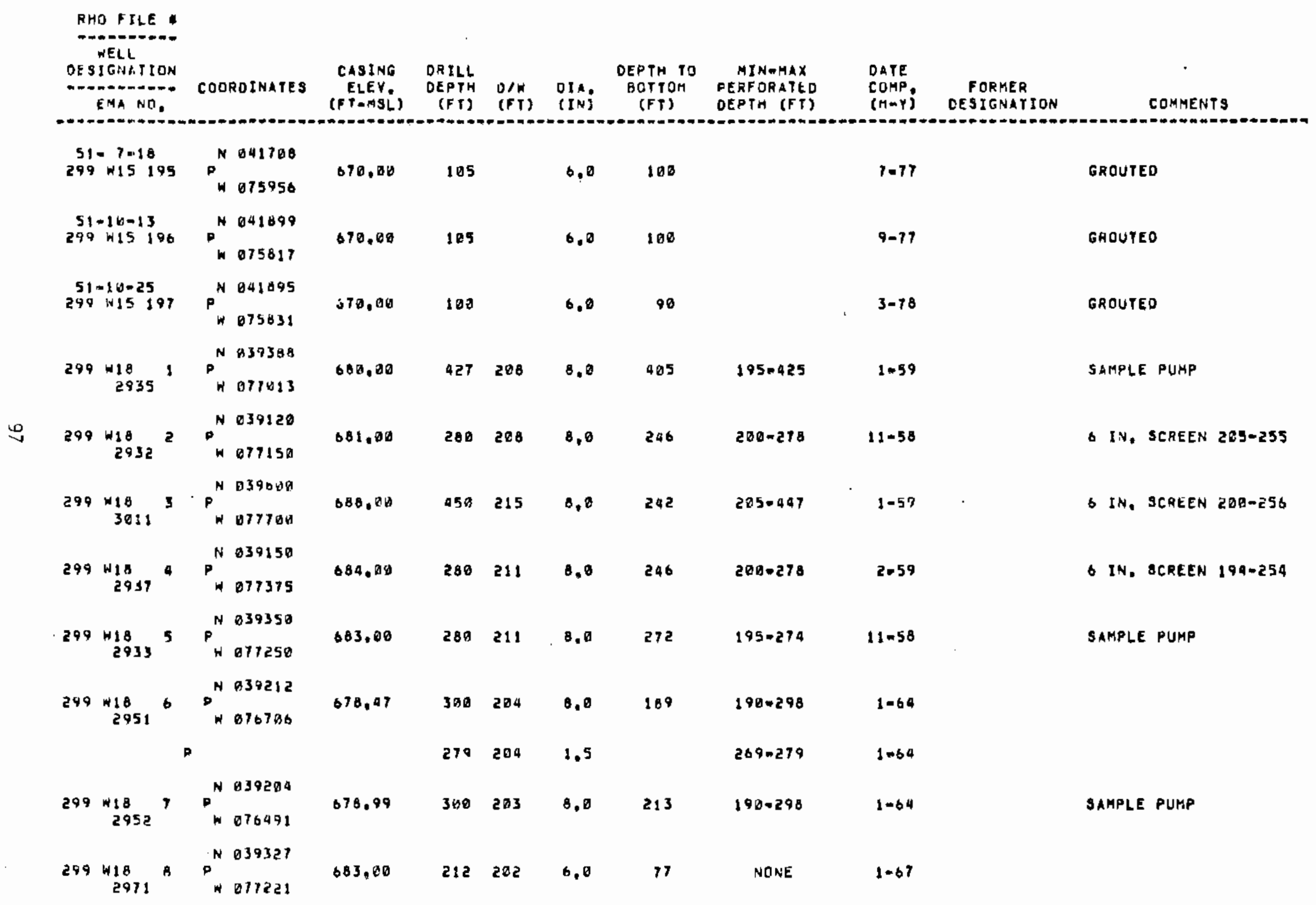




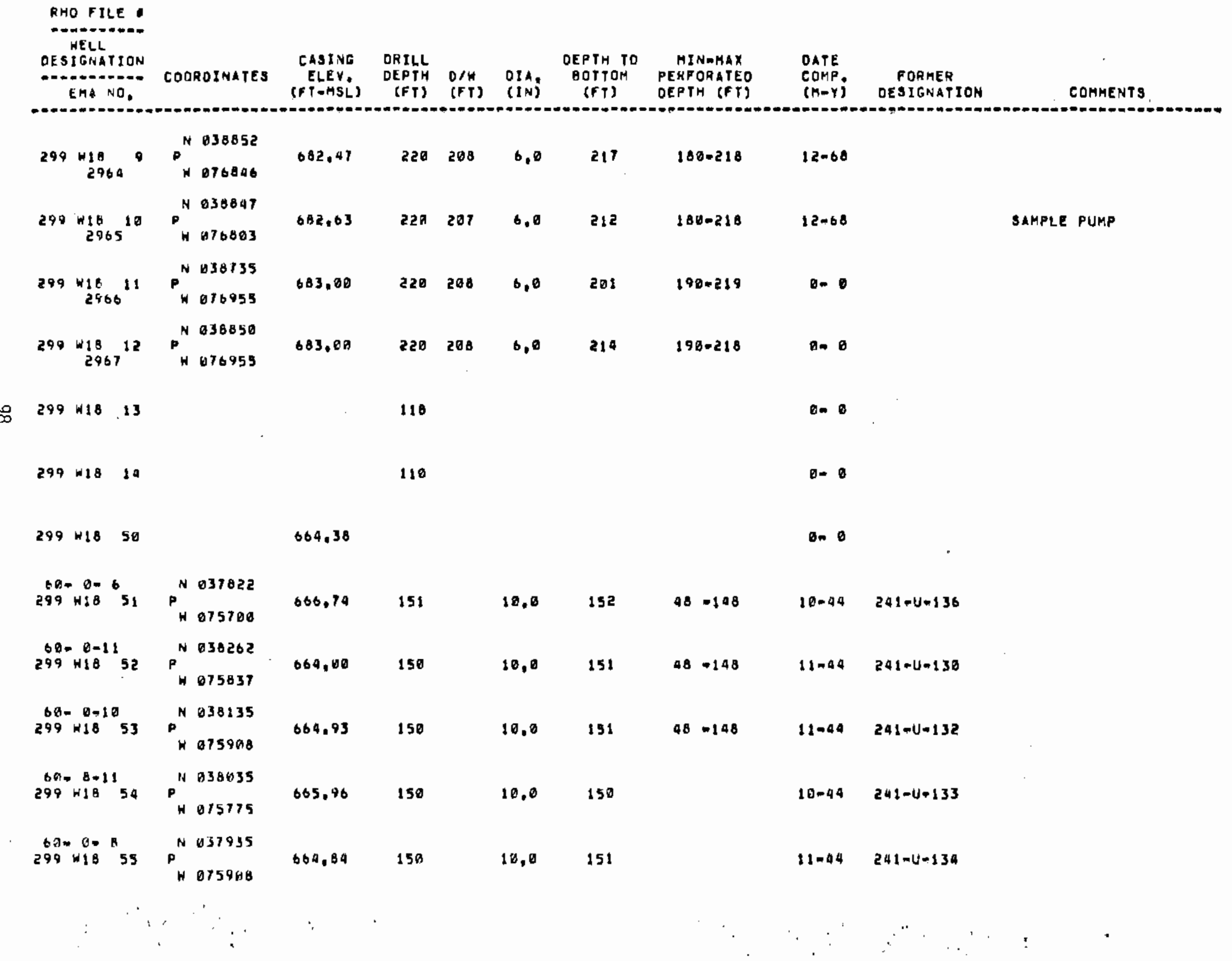




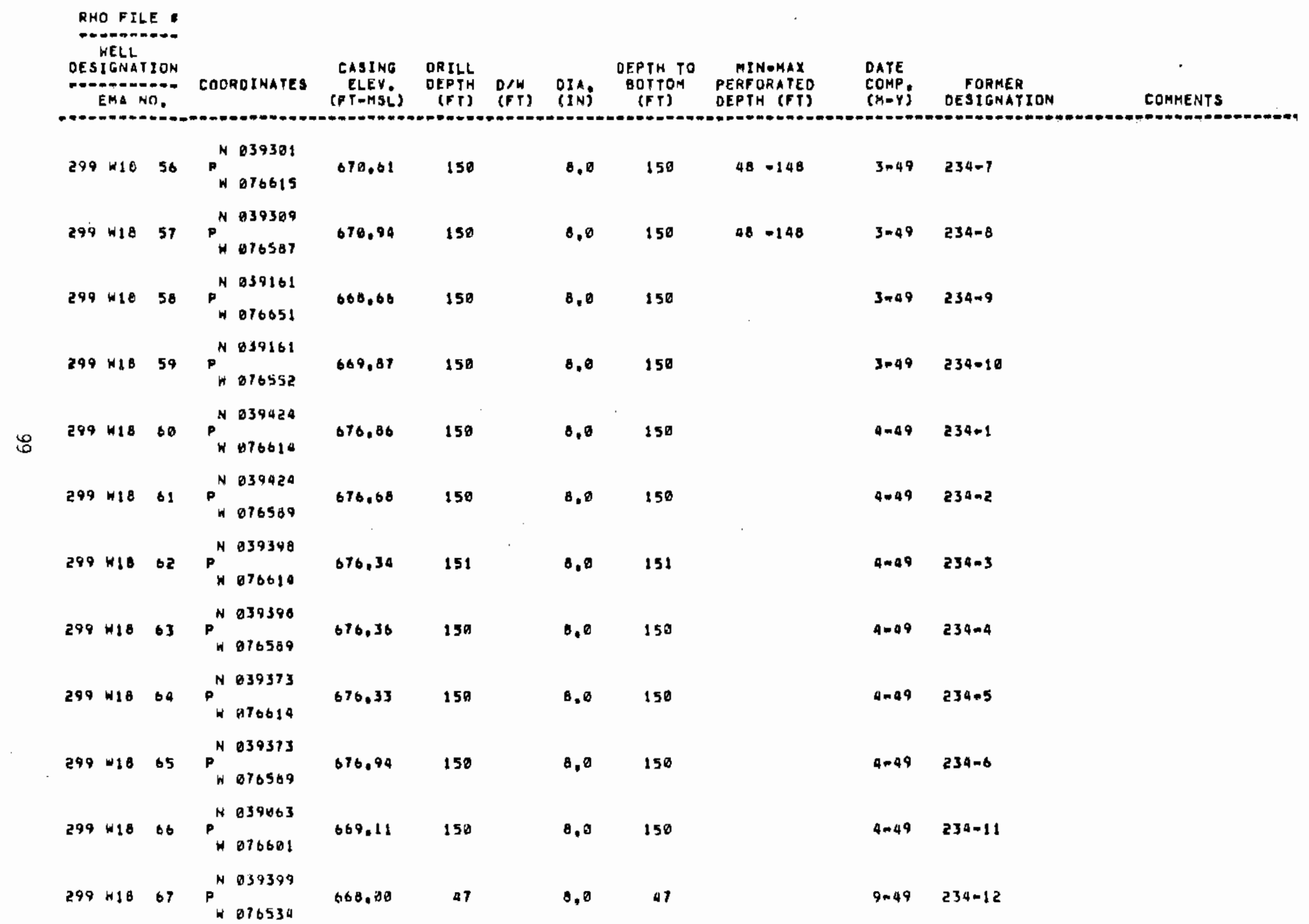


RHO PILE

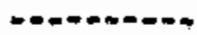

NELL

Destuination

EMA NO,

COORDINATES

ELEY DRILL

DRILL
DEPTH O/H DIA. DEPTH TO MITOM PERFORATED DEPTH (FT)

DATE

COMP. FORMER

(HAY) DESIGNATION

CDMMENTS

H 639371

299 W18 68 P

$668,00 \quad 46 \quad 8,0$

$9-49 \quad 234 * 13$

299 W18 69

50

6,0

$1-67$

290030395

W 077206

20

6.0

$3=67$

299 H16 71 P ${ }^{N 039346}$

20

6.0

$3-67$

占 299 W18 72 P $P^{N} 039498$

26

6,0

$3=67$

299 H10 $73 P_{H}^{N 039202}$

25

6.0

$3 * 67$

$29941874 P^{N 039108}$

25

6,0

3.67

$299 H 18 \quad 75 P^{N 039375}$

2!

6.0

$3=67$

$299418 \quad 16 p^{N 939318}$

19

6.0

$3-67$

$299 \quad 18 \quad 77 P_{H}^{N 039273}$

$3=67$

$299 H 1078 \quad P^{N} 039308$

$3=67$

$299 H 18 \quad 19 P^{N} \operatorname{0s9274}$
Ho70594

25

6,0

$3=67$ 


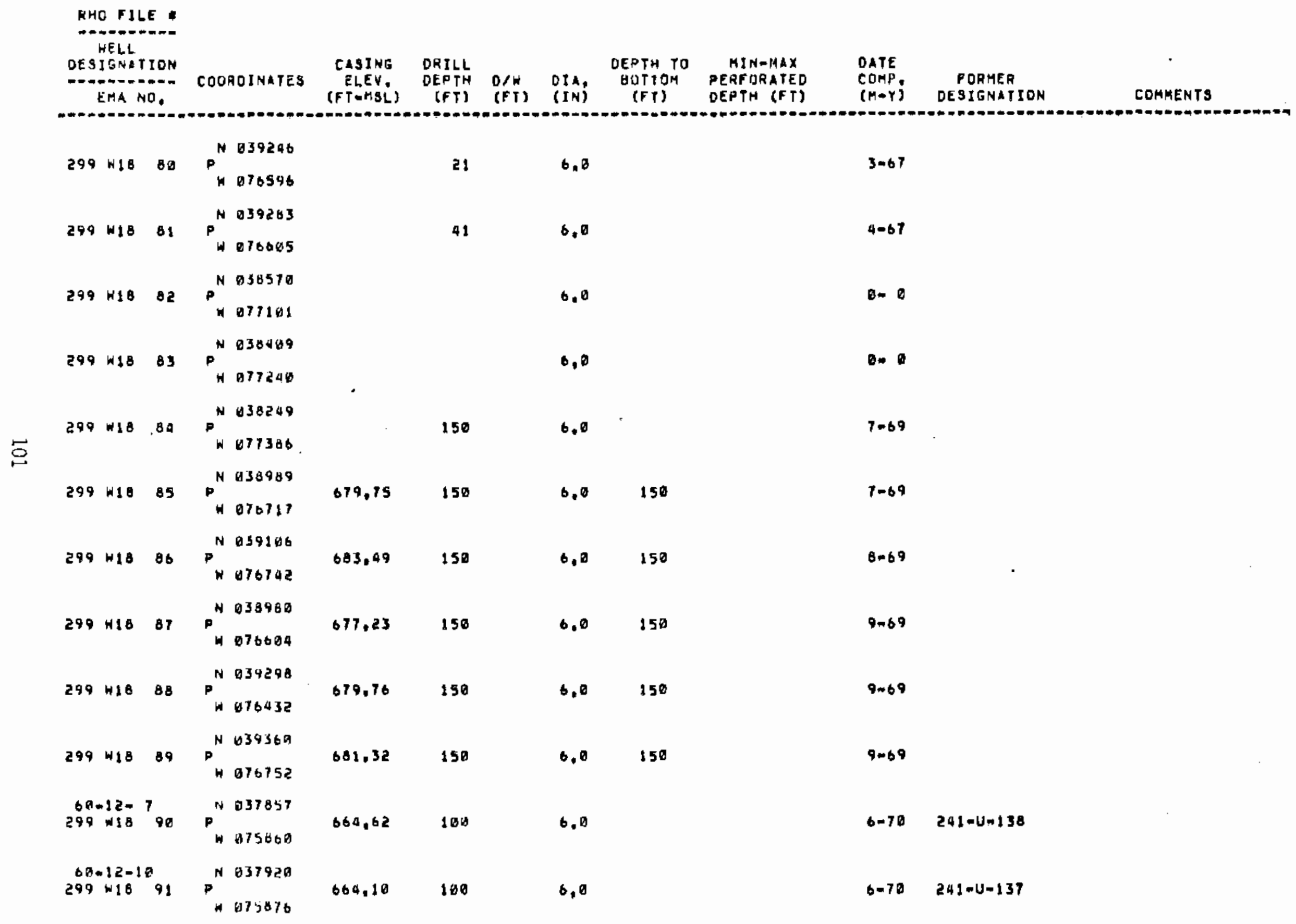




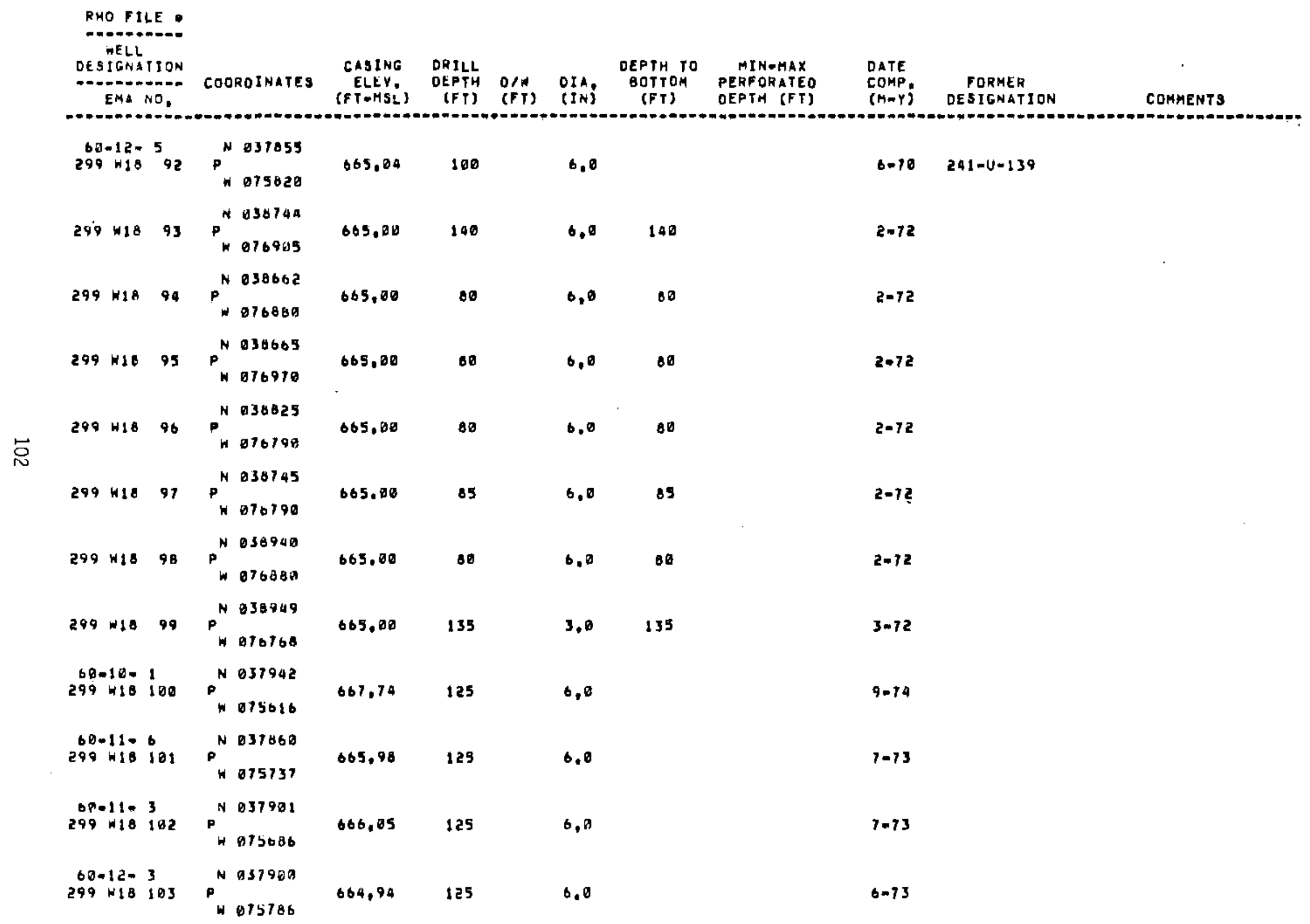




\begin{tabular}{|c|c|c|c|c|c|c|c|c|c|}
\hline OESIGNATION & COURDINATES & $\begin{array}{l}\text { CASING } \\
\text { ELEY. } \\
\text { (FTEMSi) }\end{array}$ & $\begin{array}{l}\text { DNILL } \\
\text { DEPTH } \\
\text { (FT) }\end{array}$ & $\begin{array}{l}O / W \\
(F T)\end{array}$ & $\begin{array}{l}\text { DIA: } \\
\text { (IN) }\end{array}$ & $\begin{array}{l}\text { DEPTH TO } \\
\text { BOTTOM } \\
\text { (FT) }\end{array}$ & $\begin{array}{l}\text { MINGHAX } \\
\text { PERF ORATED } \\
\text { OEPTH (FT) }\end{array}$ & $\begin{array}{l}\text { OATE } \\
\text { COHPP, } \\
(M-Y)\end{array}$ & $\begin{array}{l}\text { FORMER } \\
\text { DESIGNATION }\end{array}$ \\
\hline $\begin{array}{rl}69-10=5 \\
299+18 & 100\end{array}$ & $\begin{array}{l}P^{N} 437857 \\
N 075612\end{array}$ & 667,43 & 125 & & 6,0 & & & 6.74 & \\
\hline $\begin{array}{l}6 P+11-12 \\
299+18105\end{array}$ & $\begin{array}{l}P^{N} 837947 \\
W 075737\end{array}$ & 665.92 & 125 & & 6,0 & & & 6.73 & \\
\hline $299+18 \quad 106$ & & & & & & & & 0.0 & \\
\hline $299 W 16 \quad 107$ & & & 105 & & 6.0 & & & $1-16$ & \\
\hline $290 \times 18 \quad 108$ & & & & & & & & 0.0 & \\
\hline $\begin{array}{l}69-11 \pi 5 \\
299 \text { WIA } 100\end{array}$ & $\begin{array}{l}P^{N} 037897 \\
W 075717\end{array}$ & 665,95 & 123 & & 6.0 & & & $4+74$ & $\cdot$ \\
\hline $\begin{array}{rl}60-11 \% & 7 \\
299418 & 110\end{array}$ & $\begin{array}{l}P^{M} 037857 \\
W 075763\end{array}$ & 665.65 & 125 & & 6,0 & & & 5.74 & \\
\hline 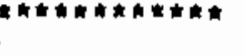 & & & & & & & & & \\
\hline $299 \times 18 \quad 112$ & & & & & & & & $8-0$ & \\
\hline $\begin{array}{rl}60-12-1 \\
299+18 & 113\end{array}$ & $\begin{array}{l}P_{N}^{N} 03794075817 \\
N 07\end{array}$ & 664.63 & 125 & & 0.0 & & & 7.74 & \\
\hline 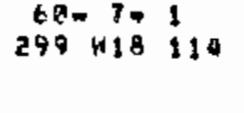 & $\begin{array}{l}P^{N} 030043 \\
W 075617\end{array}$ & 665.00 & 105 & & 6,0 & & & $1-76$ & \\
\hline $299118 \quad 115$ & $\begin{array}{l}P^{N} 837976 \\
H 273692\end{array}$ & 666,10 & 125 & & 6,0 & & & $3-74$ & \\
\hline
\end{tabular}


RHO File

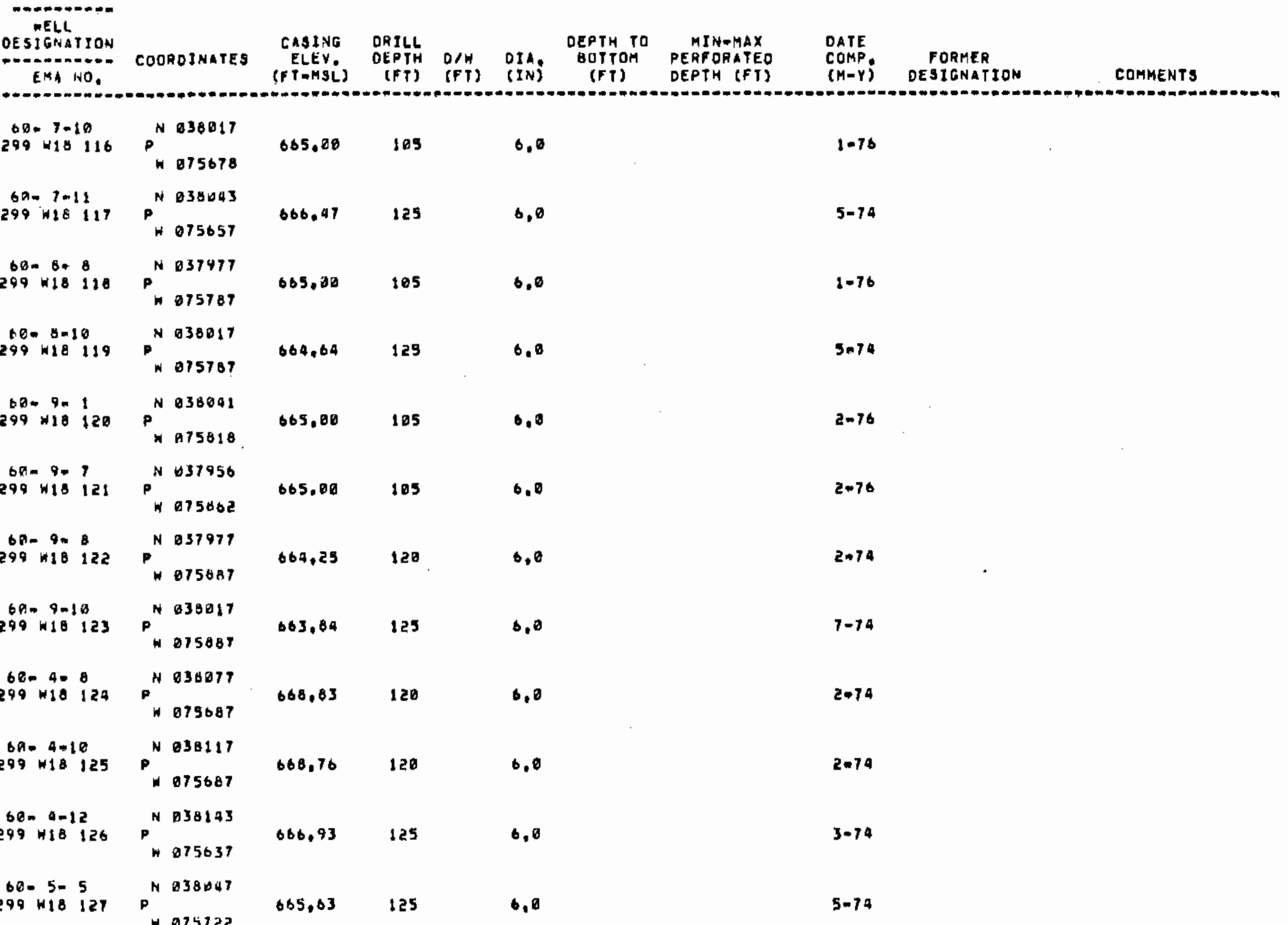




\begin{tabular}{|c|c|c|c|c|c|c|c|c|c|}
\hline DESIGNATION & CDORDINATES & $\begin{array}{l}\text { CASING } \\
\text { ELEY } \\
\text { (FTAMSL) }\end{array}$ & $\begin{array}{l}\text { DRILL } \\
\text { DEPTH } \\
\text { (FT) }\end{array}$ & $\begin{array}{l}D / k \\
(F T)\end{array}$ & DIA; & $\begin{array}{l}\text { DEPTH FO } \\
\text { BOTYOH } \\
\text { (FT) }\end{array}$ & $\begin{array}{l}\text { MINAMAX } \\
\text { PEREORATED } \\
\text { DEPTH (FT) }\end{array}$ & $\begin{array}{l}\text { DATE } \\
\text { CQMP, } \\
\text { (M-Y) }\end{array}$ & $\begin{array}{l}\text { FORMER } \\
\text { DESIGNATION }\end{array}$ \\
\hline 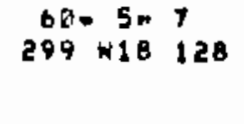 & $\begin{array}{l}P^{N} 838047 \\
W 075757\end{array}$ & 665,04 & $\$ 25$ & & 6,0 & & & $5-74$ & \\
\hline $\begin{array}{r}69-5-5 \\
299+18129\end{array}$ & $\begin{array}{l}P^{N} 038077 \\
H 075707\end{array}$ & 604.73 & 129 & & 6,0 & & & $4-74$ & \\
\hline $\begin{array}{l}60-5-10 \\
299+18130\end{array}$ & $\begin{array}{l}P_{H}^{N} 038117 \\
\text { H. }\end{array}$ & & 100 & & 6,0 & & & 5,75 & \\
\hline $\begin{array}{rl}60-60 & ? \\
299 & \text { W18 } 131\end{array}$ & $\begin{array}{l}p^{N} 038051 \\
H 075857\end{array}$ & 664,16 & 129 & & $b, 0$ & & & 7.74 & \\
\hline 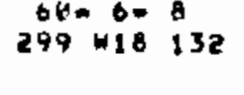 & $\begin{array}{l}P^{N} 030077 \\
N 075885\end{array}$ & & 100 & & 6,8 & & & $5-75$ & \\
\hline $\begin{array}{l}69060.10 \\
299+18133\end{array}$ & $\begin{array}{l}p^{N} 038117 \\
H 075085\end{array}$ & 669,39 & 129 & & 6.0 & & & $4-74$ & \\
\hline $299 w 18134$ & P & & 105 & & 6,0 & & & $2+76$ & \\
\hline $\begin{array}{rl}68-1 * \\
299+18 & 135\end{array}$ & $\begin{array}{l}P_{W}^{N} 0738177 \\
N 690\end{array}$ & 666.41 & 125 & & 6,8 & & & $4-74$ & \\
\hline $\begin{array}{l}60+180 \\
299-18136\end{array}$ & $\begin{array}{l}\text { p } 038217 \\
H 075687\end{array}$ & & 100 & & 6.0 & & & $3-73$ & \\
\hline $\begin{array}{c}60-2=1 \\
299+18137\end{array}$ & $\begin{array}{l}p^{N} 036244 \\
H \quad 875717\end{array}$ & 665,59 & 125 & & 6.0 & & & $4-74$ & \\
\hline 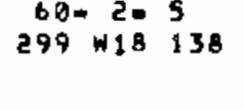 & $\begin{array}{l}P^{N} 038164 \\
N 075709\end{array}$ & & 100 & & 6.0 & & & $5+75$ & \\
\hline 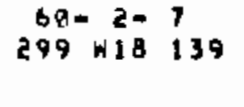 & $\begin{array}{l}\text { N } 638151 \\
\text { N075757 }\end{array}$ & 665,96 & 129 & & 6,8 & & & $3 * 74$ & \\
\hline
\end{tabular}




\begin{tabular}{|c|c|c|c|c|c|c|c|c|c|}
\hline $\begin{array}{l}\text { WELL } \\
\text { OESIGNATION } \\
\text { EMA NOA }\end{array}$ & COOROINATES & $\begin{array}{c}\text { CABING } \\
\text { ELEV } \\
(F Y \rightarrow H S L)\end{array}$ & $\begin{array}{l}\text { ORILL } \\
\text { OEPTH } \\
\text { (FT) }\end{array}$ & $\begin{array}{l}\text { OHW } \\
\text { OIA }\end{array}$ & $\begin{array}{l}\text { OEPTH TO } \\
\text { BOTTOM } \\
\text { (FT) }\end{array}$ & $\begin{array}{l}\text { MINGMAX } \\
\text { PERFORATED } \\
\text { OEPTH (FT) }\end{array}$ & $\begin{array}{l}\text { DATE } \\
\text { COMP, } \\
\text { (MAY) }\end{array}$ & $\begin{array}{l}\text { FORMER } \\
\text { OESIGNATION }\end{array}$ & COMHENTS \\
\hline 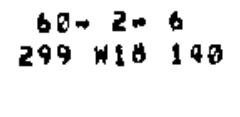 & 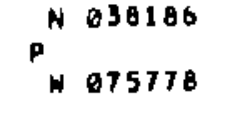 & & 105 & 6,0 & & & $2 \times 16$ & & \\
\hline $\begin{array}{l}60-2+10 \\
299 \times 18141\end{array}$ & $\begin{array}{l}P_{N}^{N} 038217 \\
075787\end{array}$ & 664,98 & 125 & 6.0 & & & $8=74$ & & \\
\hline $\begin{array}{l}60 \% 2-11 \\
299+18142\end{array}$ & $\begin{array}{l}P_{H}^{N} 0382400 \\
{ }^{2} 0757\end{array}$ & & 100 & 6.0 & & & $3-75$ & & \\
\hline $\begin{array}{l}60 \mathrm{~m} \\
290 \\
\text { w1 }\end{array}$ & $\begin{array}{l}P_{H}^{N} 038244 \\
H 075817\end{array}$ & & 100 & 6.0 & & & $3-75$ & & \\
\hline $\begin{array}{rl}64 \mathrm{Ha} & 3 \\
299 & \mathrm{~W} \\
288 & 144\end{array}$ & $\begin{array}{l}P_{H}^{N} 038151 \\
H 075822\end{array}$ & 664,56 & 125 & 6,0 & & & $4=74$ & & \\
\hline $\begin{array}{rll}629 & 30 \\
299 & 0 & 0\end{array}$ & $\begin{array}{l}P^{N} 038177 \\
{ }_{M} 078855\end{array}$ & 664,38 & 125 & 6.0 & & & $5-74$ & & \\
\hline $\begin{array}{l}6403 \times 10 \\
299 \times 18196\end{array}$ & $\begin{array}{l}P^{N} 038217 \\
N 07545\end{array}$ & & 100 & 6.5 & & & 4.75 & & \\
\hline $\begin{array}{r}60-3711 \\
299+10147\end{array}$ & $\begin{array}{l}P_{H}^{N} 038244 \\
H 075457\end{array}$ & 669,13 & 125 & 6.0 & & & $8-74$ & & \\
\hline 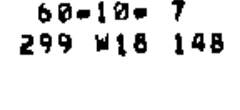 & $\begin{array}{l}P_{H}^{N} 037857 \\
H^{2} 0757\end{array}$ & 666,86 & 120 & 6.0 & & & $2 \times 74$ & & \\
\hline $299 \quad 118 \quad 149$ & P & 672.56 & 160 & 6,0 & 25 & & $9 \times 73$ & & 75 FT CEMENT PLUE \\
\hline $299+18150$ & P 076001 & 671.81 & 128 & 6.0 & 128 & & $6-73$ & & $\begin{array}{r}\text { DEEPENED 7-77 } \\
\text { GROUTED }\end{array}$ \\
\hline $299 W 1815 !$ & P & 680.64 & 15 & 10.0 & 15 & & 8.76 & & \\
\hline$:$ & . & - & & & & & & : & 2 \\
\hline
\end{tabular}




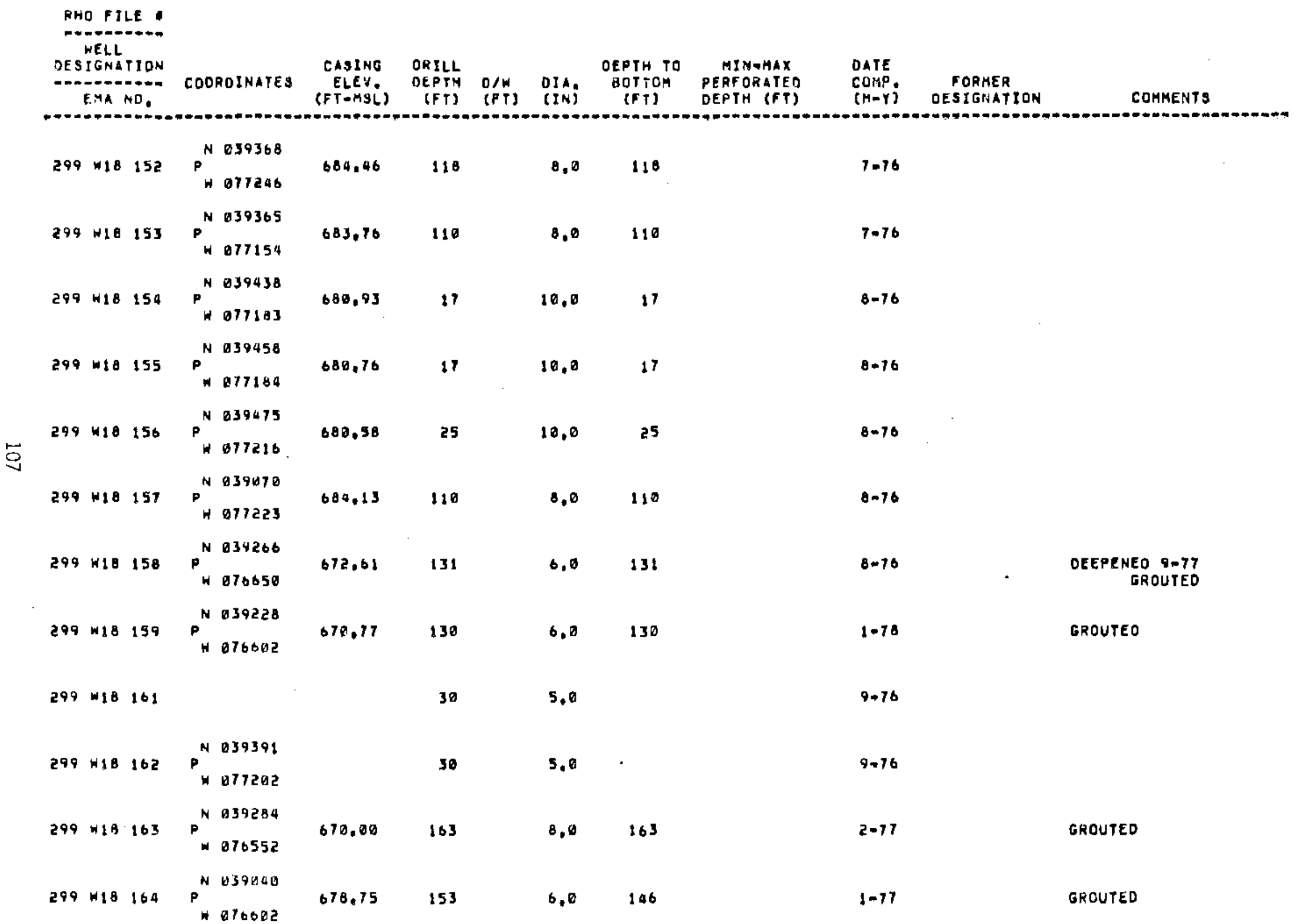




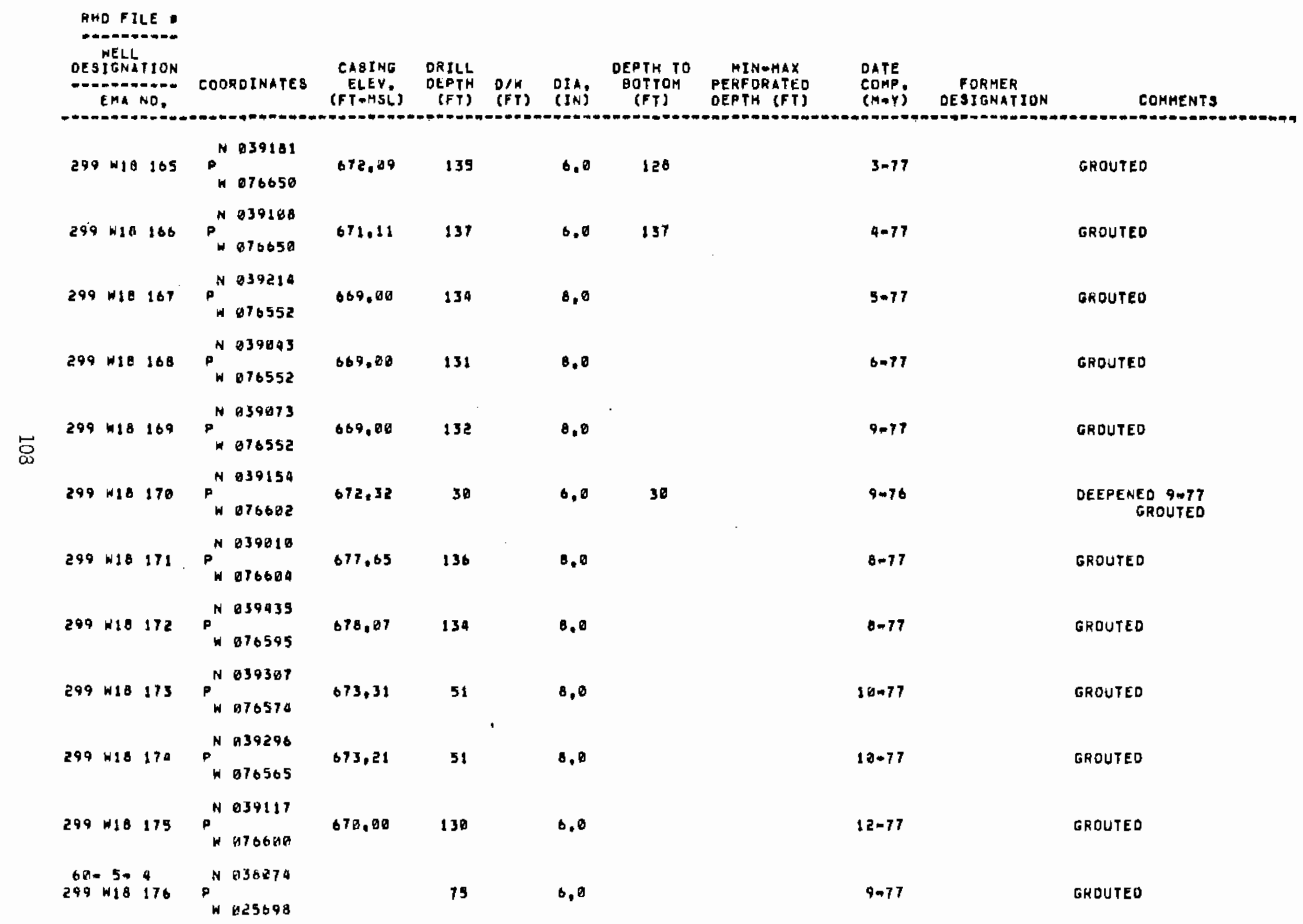




\begin{tabular}{|c|c|c|c|c|c|c|c|c|c|c|}
\hline DES: & $\begin{array}{l}\text { NELL } \\
\text { I GNATI } \\
\text { ENA NO } \\
\text { DA }\end{array}$ & & COORDINATES & $\begin{array}{c}\text { CASING } \\
\text { ELEV, } \\
\text { (FT MSL) }\end{array}$ & $\begin{array}{l}\text { ORILL } \\
\text { DEPTH } \\
\text { (FT) }\end{array}$ & (FI) & $\begin{array}{l}\text { DIA: } \\
\text { (IN) }\end{array}$ & $\begin{array}{c}\text { OEPTH TO } \\
\text { BOTTOM } \\
\text { (FT) }\end{array}$ & $\begin{array}{l}\text { MIN•MAX } \\
\text { PERFORATEO } \\
\text { OEPTH (FI) }\end{array}$ & $\begin{array}{l}\text { DATE } \\
\text { COKP, } \\
(H-Y)\end{array}$ \\
\hline \multirow[t]{3}{*}{299} & $\begin{array}{r}19 \\
2423\end{array}$ & 1 & $\begin{array}{l}P_{N}^{N 037613} \\
N 875491\end{array}$ & 673.77 & 301 & $2 a !$ & 8,1 & 200 & $178-299$ & $5-57$ \\
\hline & & 0 & 0 & 674,004 & 249 & 196 & 1,5 & & $178-196$ & $5=65$ \\
\hline & 2947 & P & b & 674.06 & 248 & 281 & 1,5 & 248 & $228-208$ & $\{1-6\}$ \\
\hline 299 & $\begin{array}{l}119 \\
2928\end{array}$ & 2 & $\begin{array}{l}P_{N 073600}^{N} 03680 \\
N 07300\end{array}$ & 594,04 & 300 & 232 & 8,0 & 240 & $235=295$ & 8.57 \\
\hline 249 & $\begin{array}{l}119 \\
2991\end{array}$ & 3 & P & 095,12 & 301 & 225 & 8,0 & 244 & $238-280$ & $9 m 57$ \\
\hline \multirow[t]{6}{*}{299} & $\begin{array}{l}119 \\
2938\end{array}$ & 4 & ${ }^{P} \quad 571999$ & 715,26 & 556 & 255 & 0.0 & 350 & $255=535$ & $2-60$ \\
\hline & & 0 & 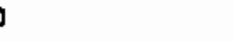 & 715,92 & 280 & 253 & 1.5 & & $260-280$ & $5-65$ \\
\hline & & $\mathbf{P}$ & 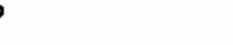 & 715,52 & 535 & 256 & 1.5 & 464 & 516.535 & $6-64$ \\
\hline & & 0 & 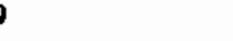 & 715,52 & 981 & 256 & 1,5 & 4 ดข & 456.481 & $6-64$ \\
\hline & & h & 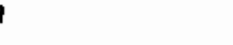 & 715,52 & 421 & 256 & 1.5 & 308 & $386 \cdot 411$ & 6.64 \\
\hline & & s & 3 & & 370 & 256 & & & $345-370$ & $D=0$ \\
\hline 299 & $\begin{array}{l}19 \\
2968\end{array}$ & 5 & $\begin{array}{l}P_{W 074605}^{N} 036850 \\
{ }^{2}\end{array}$ & 760.00 & 235 & 215 & 6.0 & 230 & $205 * 230$ & $11-68$ \\
\hline 299 & 119 & 6 & $P_{W}=74718$ & 708.00 & 422 & 216 & 6,0 & a18 & $380=411$ & $12-68$ \\
\hline 299 & $\begin{array}{l}119 \\
2970\end{array}$ & 7 & $P_{H} 074125$ & 706.00 & 235 & 217 & 6,0 & 223 & $200-233$ & $11-88$ \\
\hline 299 & $W 19$ & 8 & $P_{n} 073260$ & & 585 & 248 & 4,0 & & NONE & $6-71$ \\
\hline
\end{tabular}




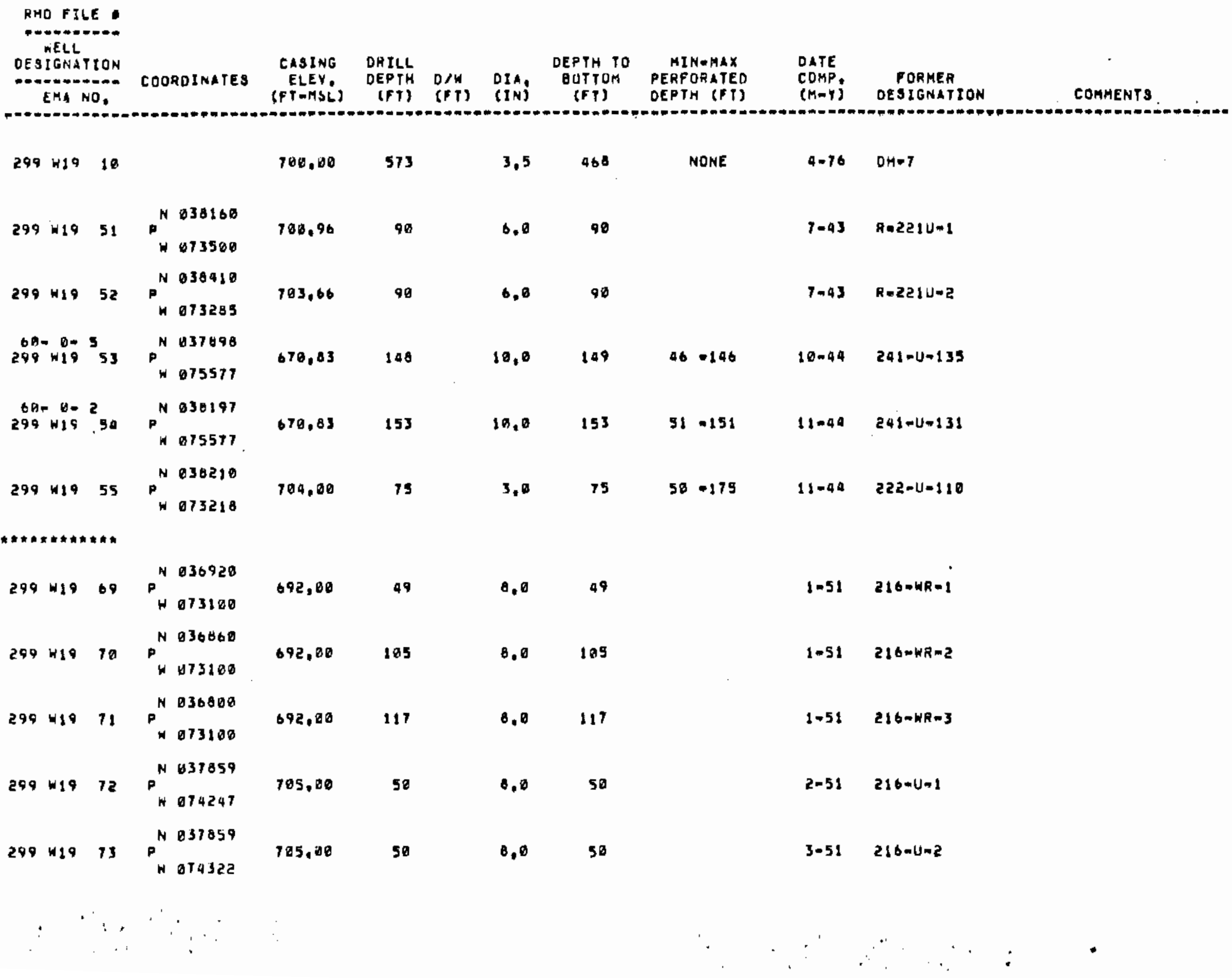




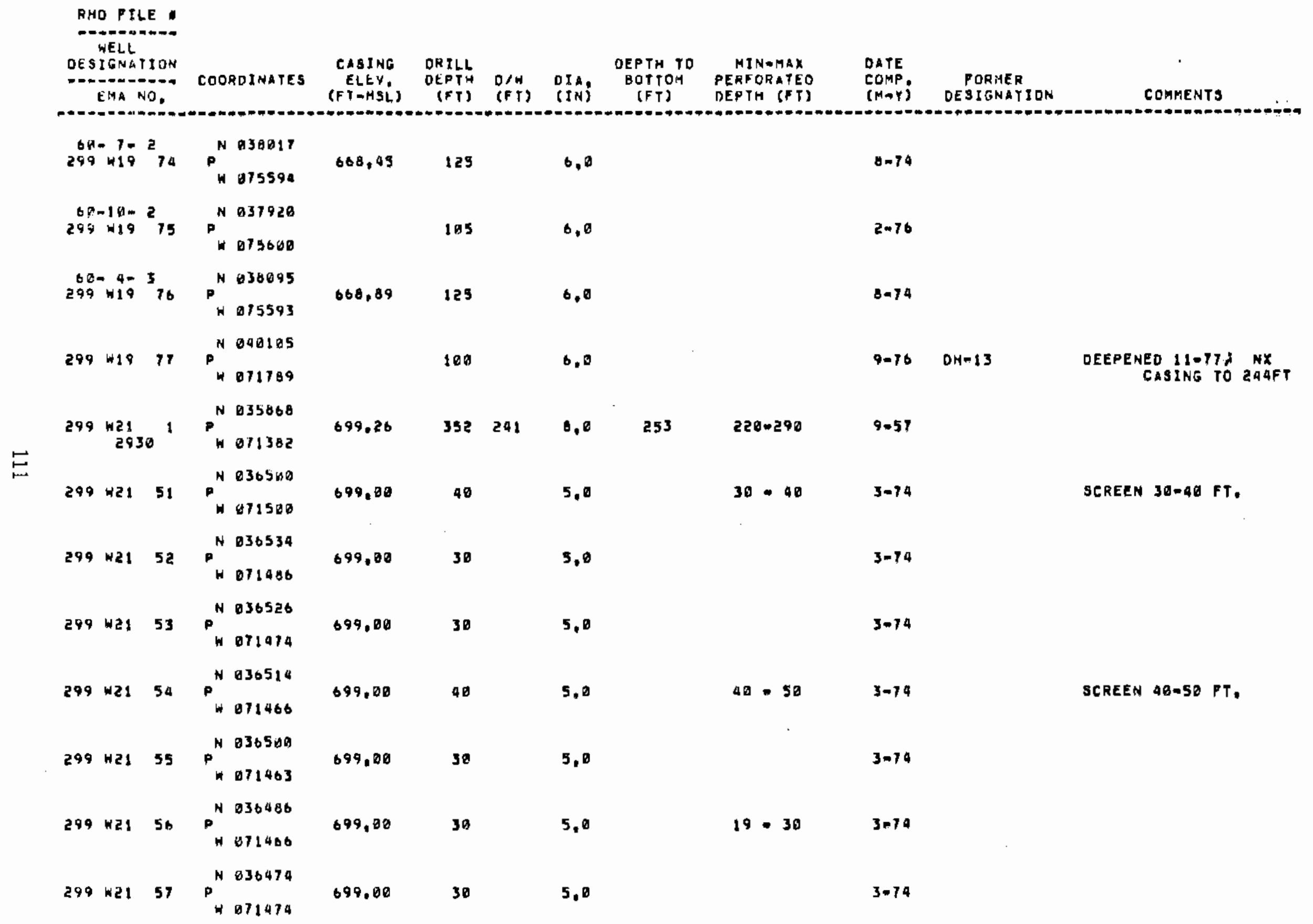




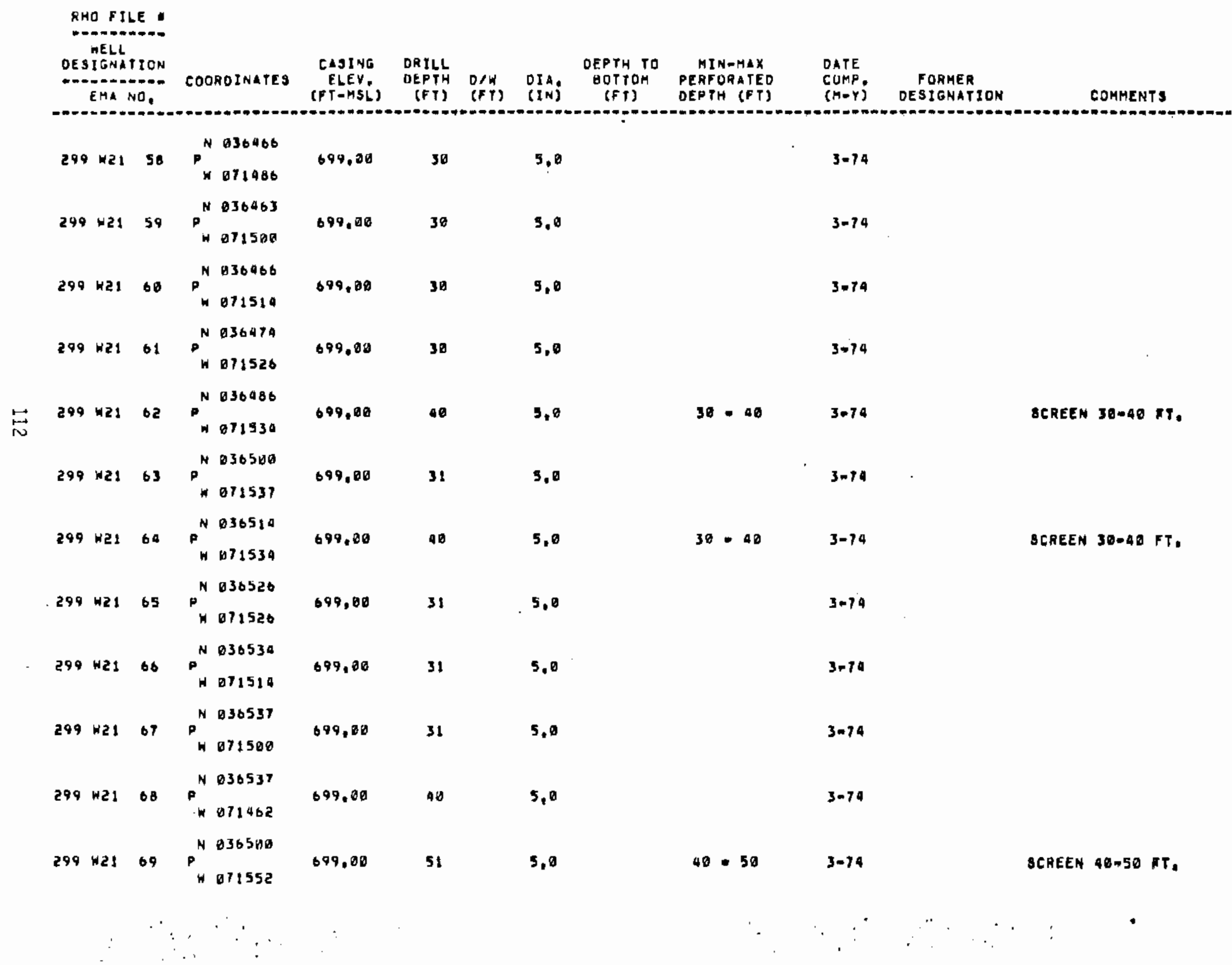




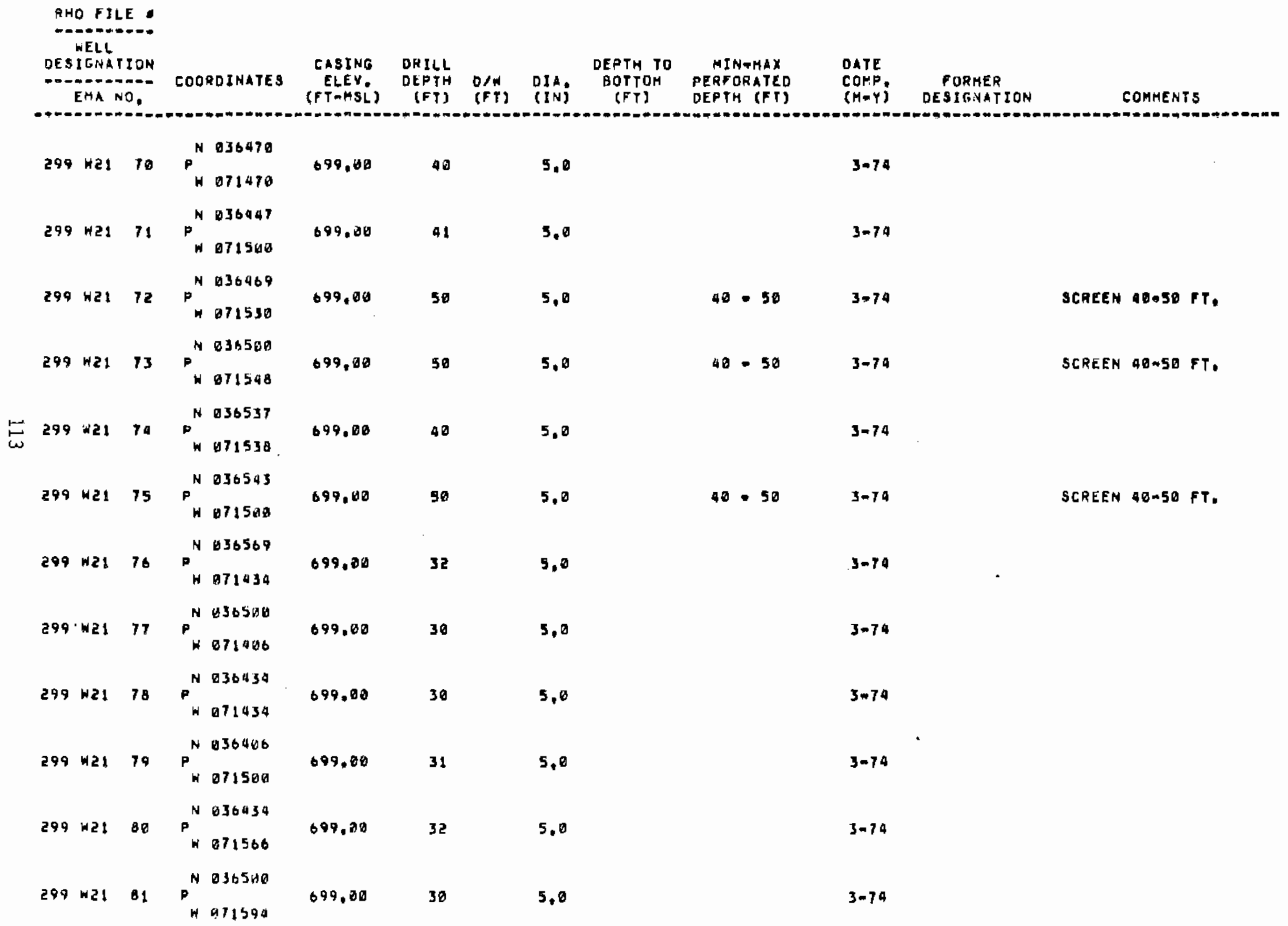


RHO FILE

RHO

$$
\text { HEL: }
$$

DESIGNITIDN OEgICNaTION EMA NO,

COQRDINATES

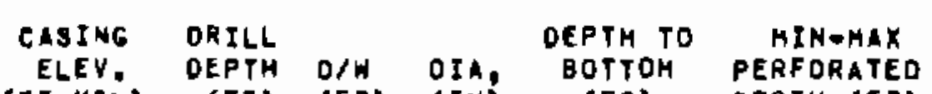
(FT) (FT) (IN) C (FT) DEPTH (FT)

DATE

CDMP. FORMER

(M-Y) OESIGNATION

COMHENTS

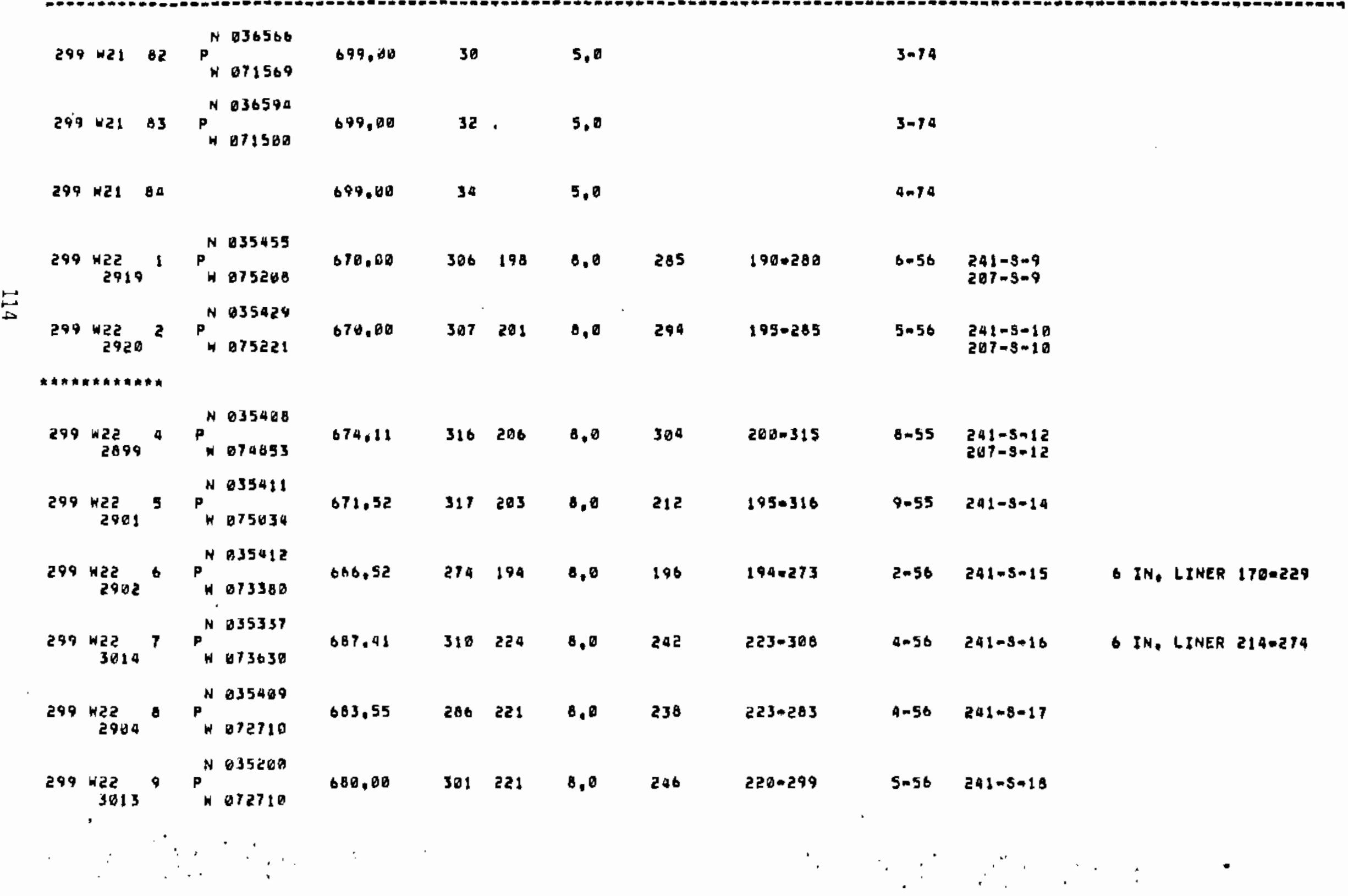




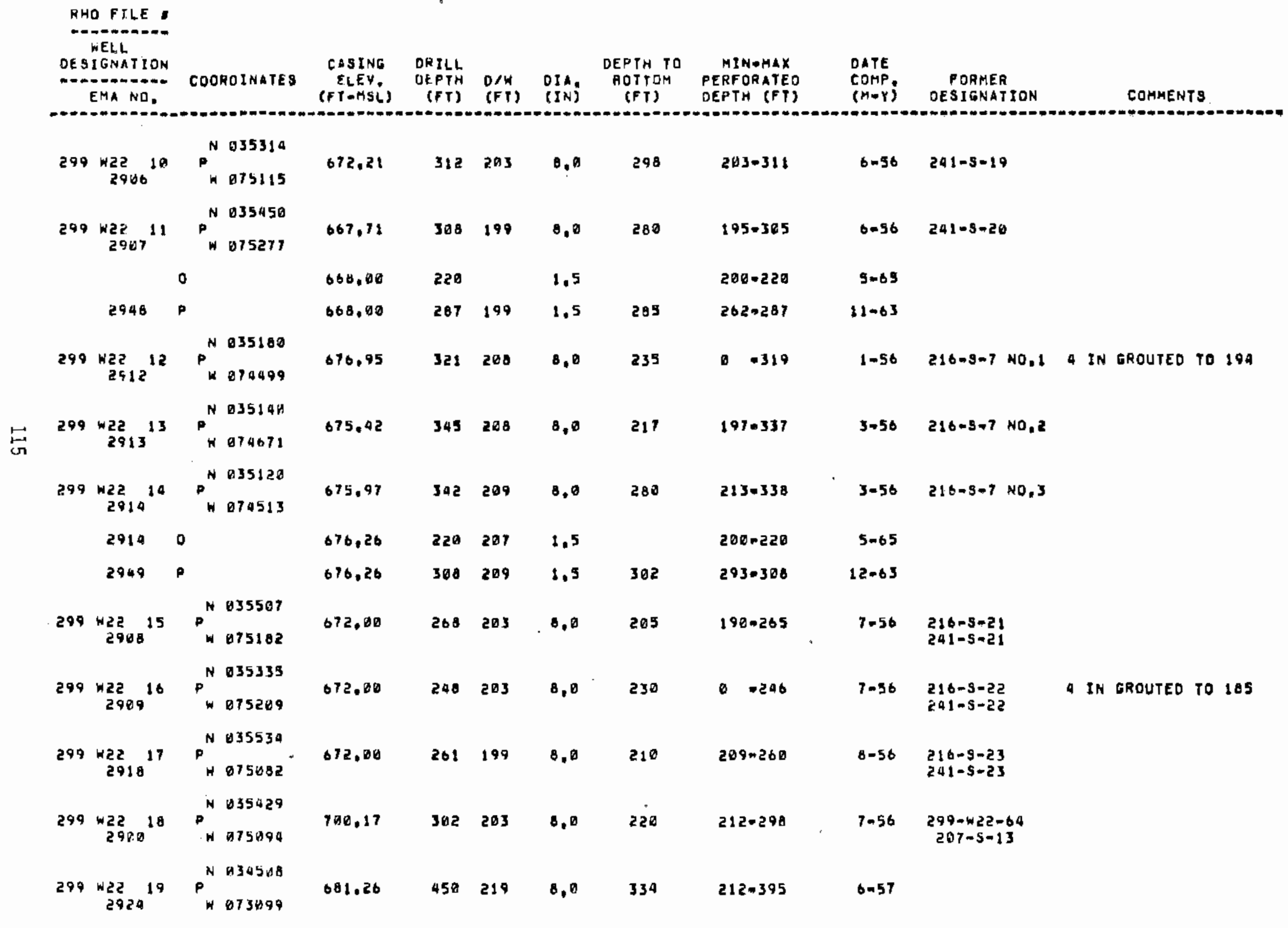




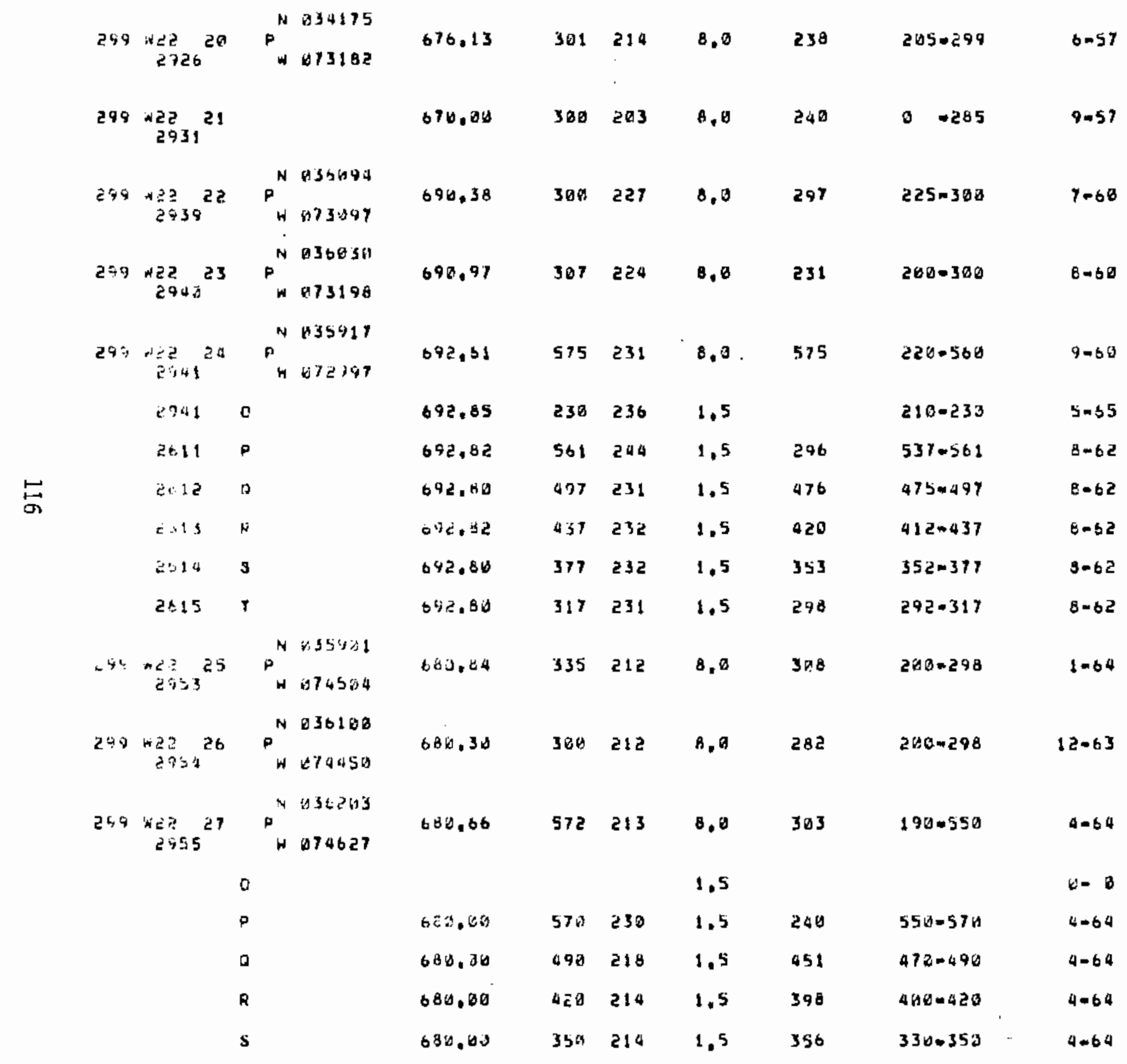




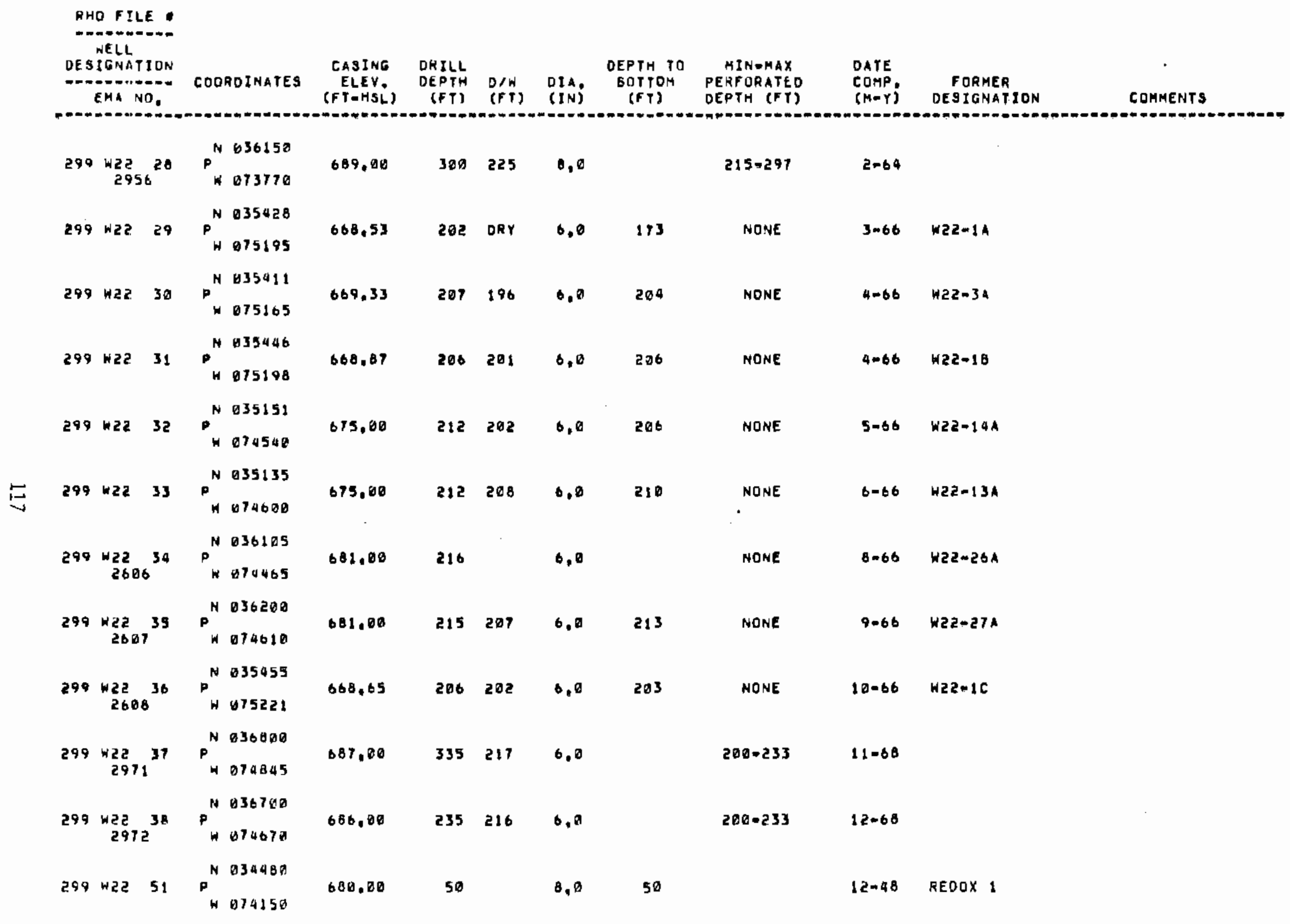




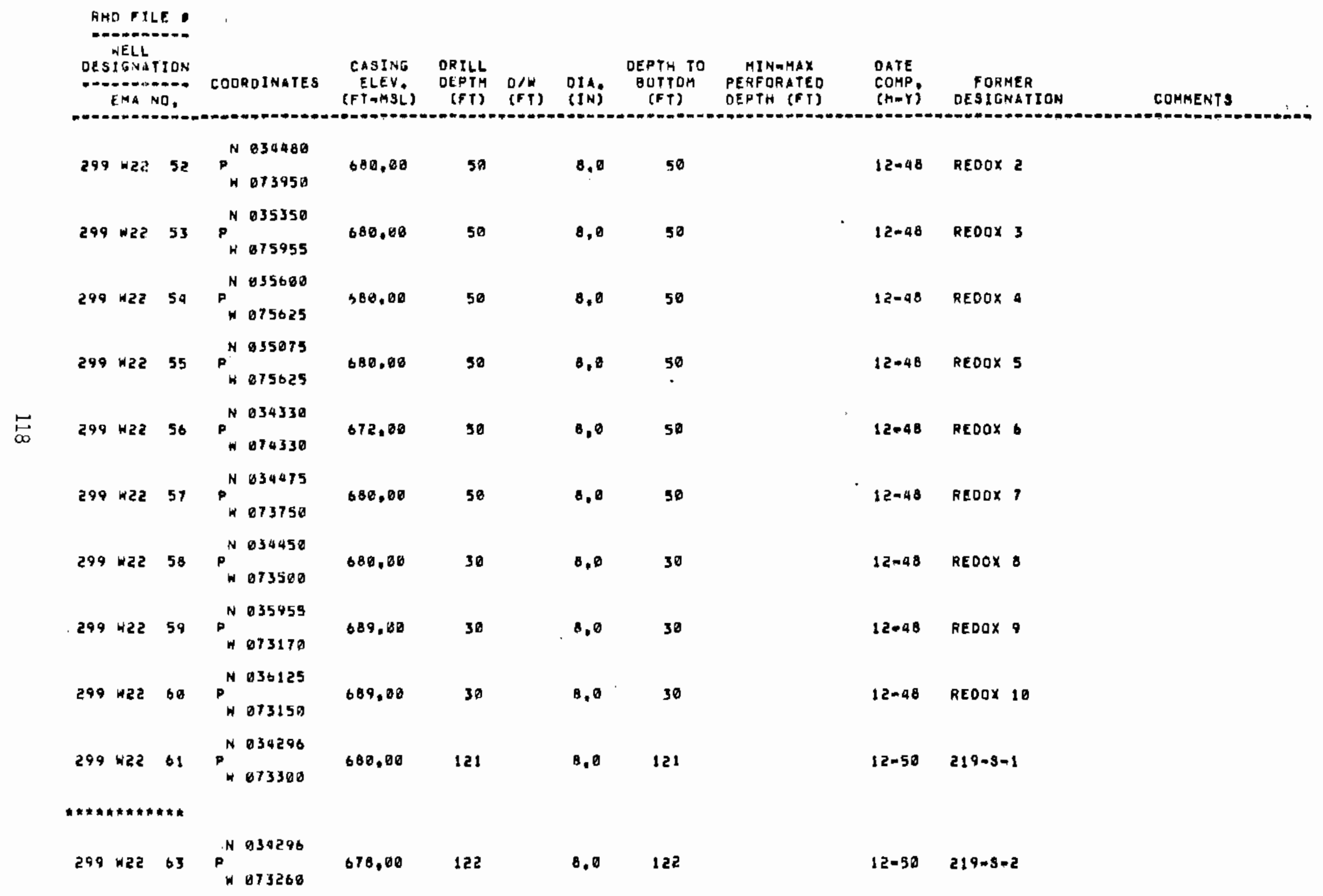




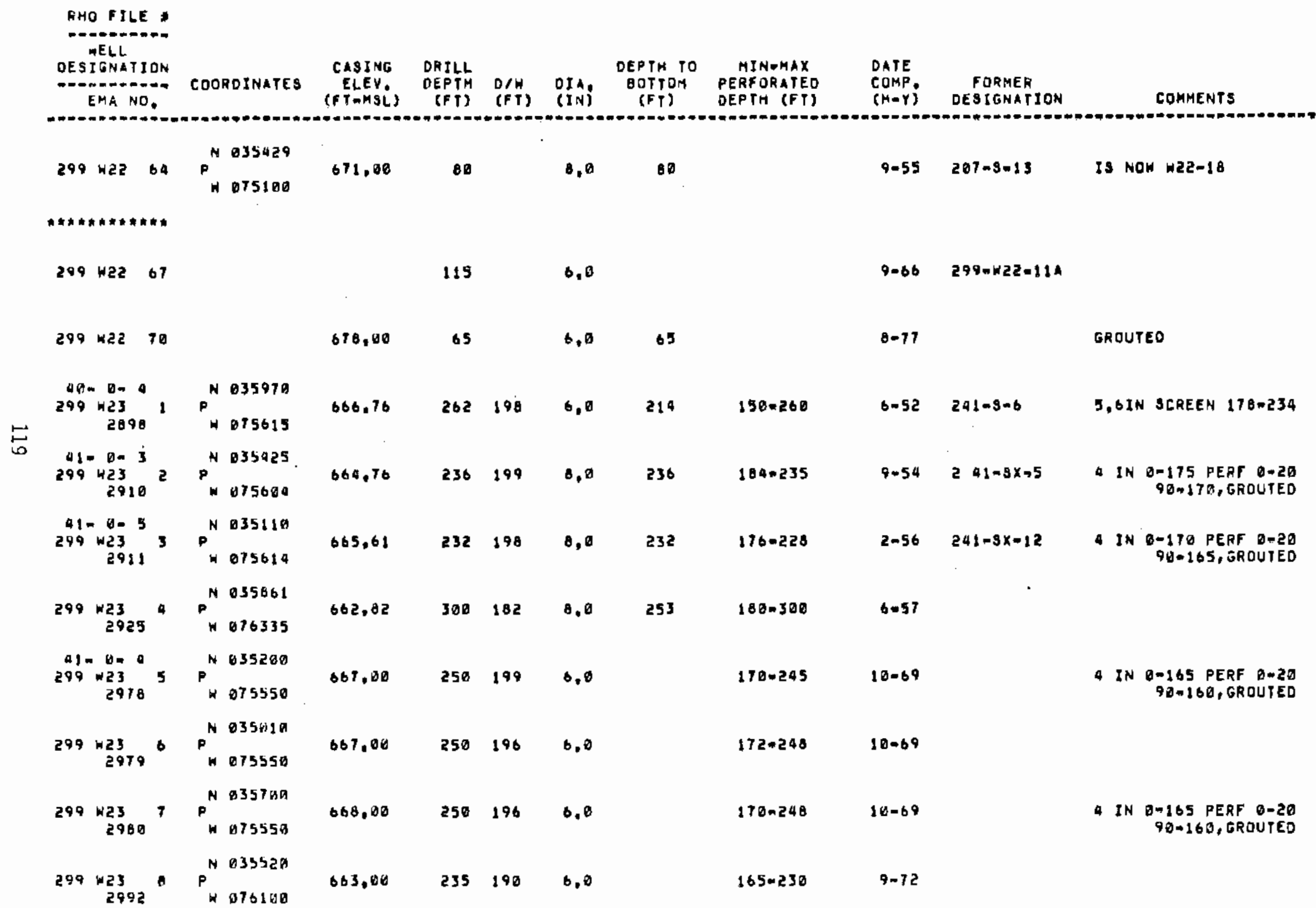




\begin{tabular}{|c|c|c|c|c|c|c|c|c|c|c|}
\hline $\begin{array}{c}\text { WELL } \\
\text { DESIGNATION } \\
\text { EMANO }\end{array}$ & CODROINATES & $\begin{array}{c}\text { CASING } \\
\text { ELEY } \\
\text { (FTIMSL) }\end{array}$ & $\begin{array}{l}\text { DRILL } \\
\text { DEPTH } \\
\text { (FT) }\end{array}$ & $(F / W)$ & DIANF & $\begin{array}{l}\text { DEPTH YO } \\
\text { BOTYOH } \\
\text { (FT) }\end{array}$ & $\begin{array}{l}\text { MINAMAX } \\
\text { PERFORATED } \\
\text { OEPTH IFT }\end{array}$ & $\begin{array}{l}\text { DATE } \\
\text { COMPP } \\
(M-Y)\end{array}$ & $\begin{array}{l}\text { FORMER } \\
\text { DESIGNATION }\end{array}$ & COMAENTS \\
\hline $299+239$ & $\begin{array}{l}{ }^{N} 035400 \\
N 070300\end{array}$ & 664.50 & 235 & 192 & 0,0 & 230 & $164-230$ & $8-72$ & & \\
\hline $299 \operatorname{was}_{2994} 10$ & ${ }^{N} 076535$ & 664,71 & 235 & 191 & 6,0 & 224 & $165 * 238$ & $18-72$ & & \\
\hline $\begin{array}{c}299 \times 23 \\
2995^{11}\end{array}$ & P & 664,14 & 235 & 187 & 0.0 & 227 & $165-239$ & $11-72$ & & \\
\hline $\begin{array}{r}48-10-1 \\
299 \times 2312\end{array}$ & $\begin{array}{l}P_{N}^{N} 035969 \\
N^{2} 075625\end{array}$ & 657.08 & 265 & & 6.0 & & 1890230 & $B-\theta$ & & $\begin{array}{r}4 \text { IN } 0-175 \text { PERF O-26 } \\
96-176, G R O U T E D\end{array}$ \\
\hline 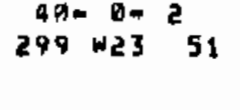 & $\begin{array}{l}P_{W}^{N} 036175 \\
P^{N} 075615\end{array}$ & 666.83 & 150 & & 6,0 & 150 & $40-100$ & $3-52$ & $2 a \mid-s-1$ & \\
\hline 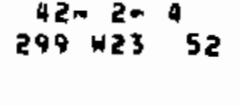 & $\begin{array}{l}P_{W 075722}^{N} 030186 \\
W 07\end{array}$ & 663,00 & 150 & & 6.3 & 150 & $40-100$ & 3.52 & $241-5-2$ & \\
\hline $\begin{array}{rl}90-3-3 & 9 \\
299 & \text { w23 } 53\end{array}$ & $\begin{array}{l}P^{N} 036186 \\
N 075627\end{array}$ & 663,98 & 150 & & 6,0 & 150 & $40-100$ & $3-52$ & $24:-5-3$ & \\
\hline $296 \times 29^{5} 3^{5} 54$ & $\begin{array}{l}P^{N} 930082 \\
H 075722\end{array}$ & 663.08 & 150 & & 6.6 & 150 & $40-100$ & $4-52$ & $241-5=4$ & \\
\hline $\begin{array}{c}44-6=5 \\
299 \text { แ23 } 55\end{array}$ & $\begin{array}{l}P^{N} 036882 \\
{ }_{10} 075827\end{array}$ & 663.65 & $15 \pi$ & & 6,0 & 150 & $40=100$ & $4-52$ & $241-5=9$ & \\
\hline $290-1006$ & $\begin{array}{l}P_{N}^{N} 035875 \\
N 075722\end{array}$ & 663,00 & 175 & & 6,0 & 150 & $40-100$ & $4-52$ & $241-5=7$ & \\
\hline $\begin{array}{r}48-975 \\
289=235\end{array}$ & $\begin{array}{l}P^{N 035880} \\
W 075027\end{array}$ & 663.82 & 175 & & 6,0 & 150 & $40-100$ & $4-52$ & $241-3=8$ & \\
\hline $\begin{array}{l}41-6011 \\
299^{2}\end{array}$ & $\begin{array}{l}\text { N015501 } \\
\text { N075900 }\end{array}$ & 661.71 & 101 & & 8,0 & 101 & $10=99$. & $9-54$ & $2 a 1-3 x=1$ & \\
\hline
\end{tabular}




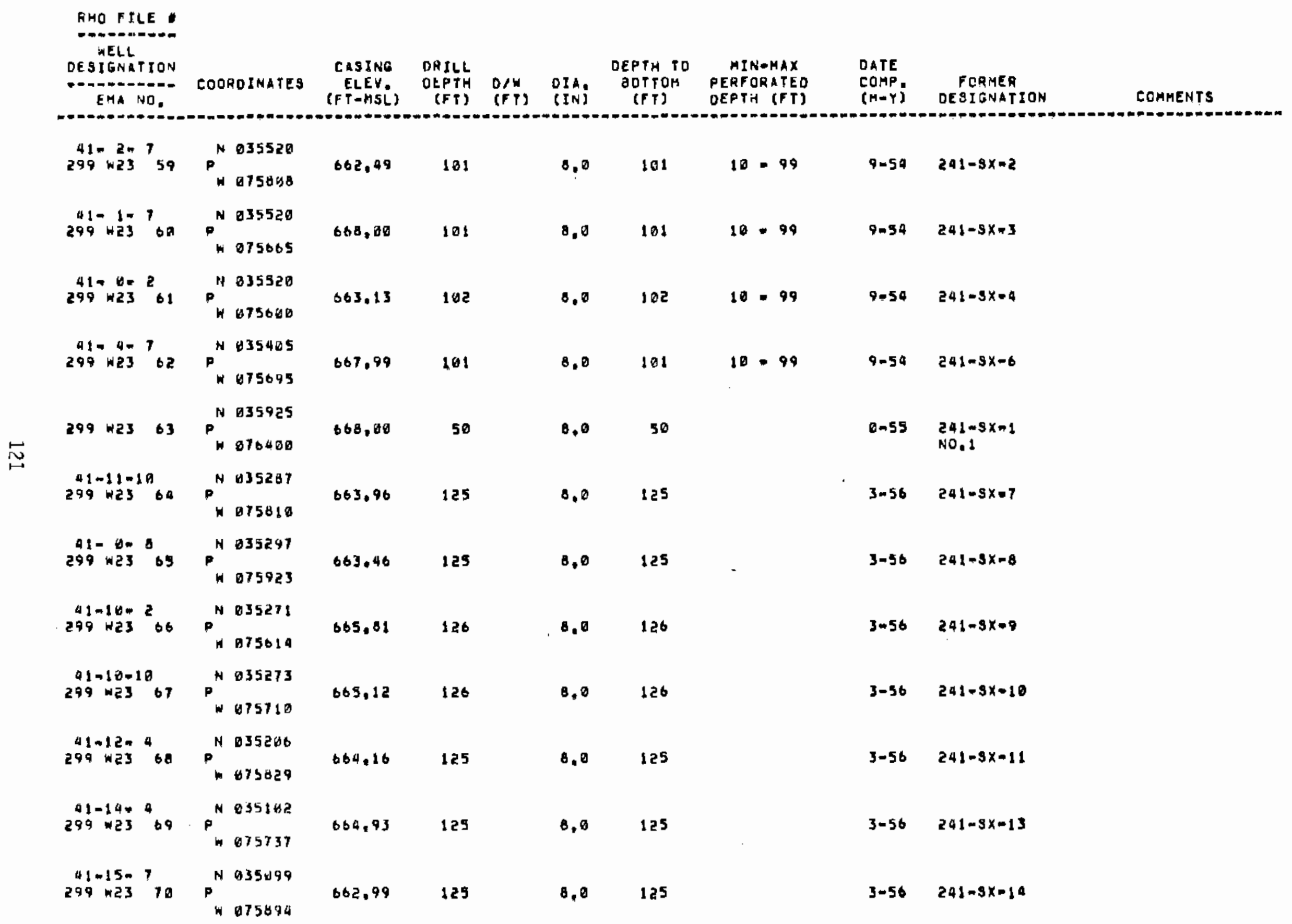




\begin{tabular}{|c|c|c|c|c|c|c|c|c|c|c|}
\hline $\begin{array}{l}\text { WELL } \\
\text { OEBIGHATION } \\
\text { EMA NO. }\end{array}$ & COORDINATES & $\begin{array}{c}\text { CASING } \\
\text { ELEY. } \\
\text { (FT^MBL) }\end{array}$ & $\begin{array}{l}\text { DRILL } \\
\text { DEPTH } \\
\text { (FT) }\end{array}$ & $\begin{array}{l}O / H \\
\text { (FT) }\end{array}$ & OIA: & $\begin{array}{l}\text { DEPTH TO } \\
\text { BDTTOH } \\
\text { (FT) }\end{array}$ & $\begin{array}{l}\text { MINAMAX } \\
\text { PERFORATEO } \\
\text { DEPTH (FT) }\end{array}$ & $\begin{array}{l}\text { DATE } \\
\text { COMP, } \\
\text { (H-Y) }\end{array}$ & $\begin{array}{l}\text { FORMER } \\
\text { OESIGNATION }\end{array}$ & COMMENTS \\
\hline 299 W3 71 & & 668.80 & 54 & & 8,0 & 54 & & 12.53 & $241-3 x=1 T, H_{0}$ & \\
\hline $\begin{array}{l}41-13-19 \\
299+2372\end{array}$ & $\begin{array}{l}P_{H}^{N 035177} \\
P_{075705}\end{array}$ & 603.01 & 100 & & 6.0 & 100 & & $10-50$ & & \\
\hline $\begin{array}{l}41-7012 \\
299 \times 23\end{array}$ & $\begin{array}{l}P^{N} 835397 \\
N \quad 875668\end{array}$ & 663,17 & 99 & & 8.0 & 85 & & $2-62$ & & $\begin{array}{c}\text { DEEPENED AND GROUTED } \\
\text { Q.TB }\end{array}$ \\
\hline $\begin{aligned} 41-7-2 \\
299+123\end{aligned}$ & $p_{W 075631}^{N 035383}$ & 663.19 & 75 & & 8.8 & 75 & & $2-62$ & & \\
\hline $\begin{array}{c}99=703 \\
299 \times 23.75\end{array}$ & $\begin{array}{l}P^{N} 035344 \\
\end{array}$ & 663.27 & 75 & & 0,0 & 15 & & $2-62$ & & \\
\hline $\begin{array}{r}91-7-5 \\
299+42376\end{array}$ & $\begin{array}{l}P_{W 075039}^{N} 035308 \\
W 070\end{array}$ & 663.54 & 75 & & 8,8 & 75 & & $2-62$ & & \\
\hline $\begin{array}{r}41-77^{7} \\
299 \times 2377\end{array}$ & $\begin{array}{l}P^{N} 0153202 \\
W 075082\end{array}$ & 663,31 & 75 & & 8,0 & 15 & & $2-62$ & - & \\
\hline $299-723^{\circ} 78$ & $\begin{array}{l}P^{N} 455332 \\
W 075711\end{array}$ & 663.17 & 73 & & 8,0 & 75 & & $2=62$ & & \\
\hline $299^{41-7+10}+23^{79}$ & $\begin{array}{l}P^{N} 035374 \\
\$ 975700\end{array}$ & 663.24 & 75 & & 8.0 & 75 & & 2.62 & & \\
\hline $\begin{array}{r}41-16-1 \\
299 \times 2380\end{array}$ & $\begin{array}{l}P^{N} 035290 \\
N 075645\end{array}$ & 663,31 & 75 & & 8,0 & 135 & & $4-73$ & & DEEPENEO $4-73$ \\
\hline $\begin{array}{c}41-10-3 \\
299+23 \text { BI }\end{array}$ & $\begin{array}{l}P^{N} 035242 \\
N 015017\end{array}$ & 663.40 & 75 & & 0,0 & 75 & & $2 m 62$ & & \\
\hline $\begin{array}{r}41-18=5 \\
299+123 \quad 02\end{array}$ & $P^{N} 0352000$ & 663.34 & 73 & & 8.0 & 75 & & $2-62$ & & \\
\hline
\end{tabular}




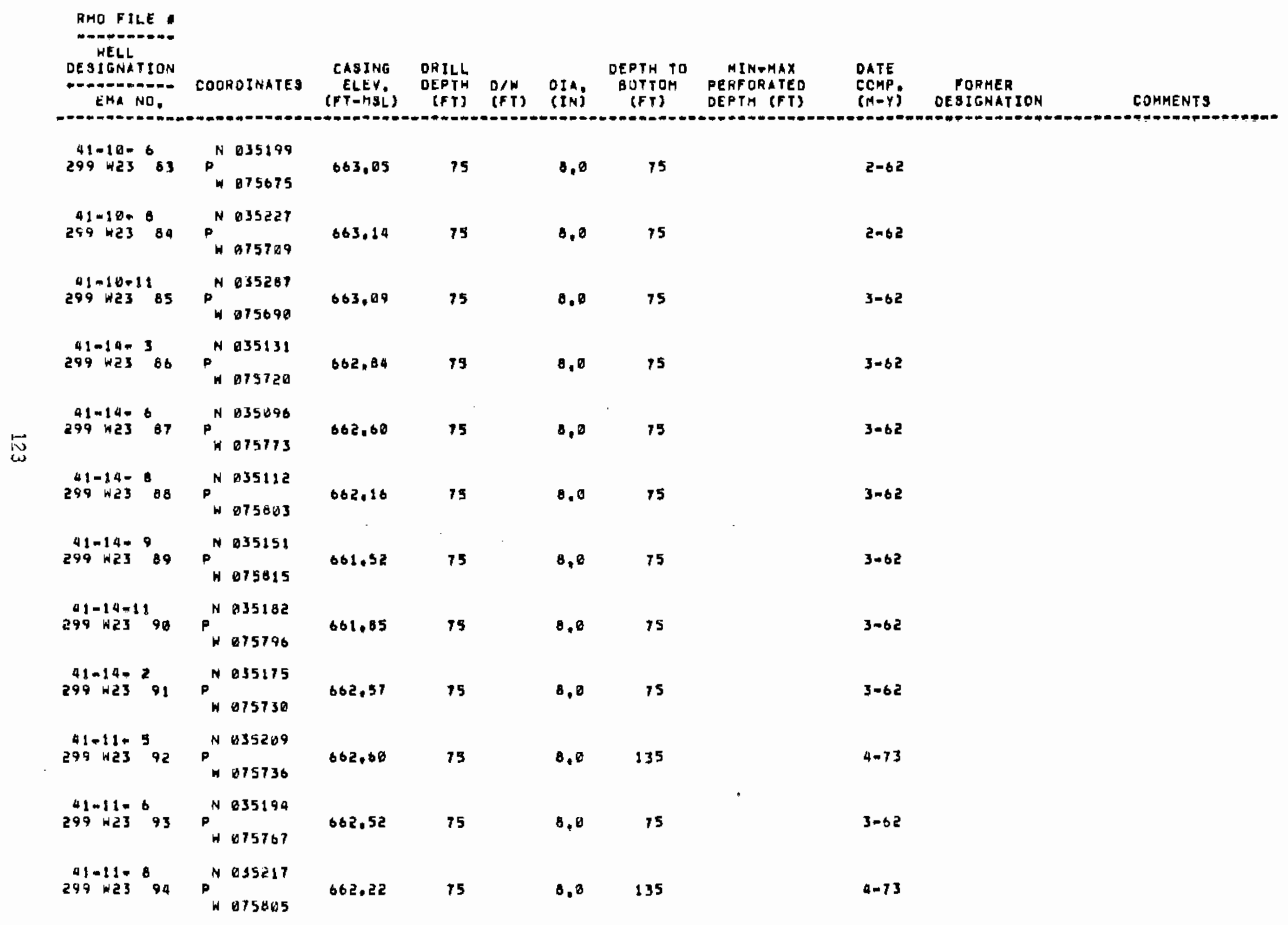


RHU FILE :

NELL

DESIGNATION

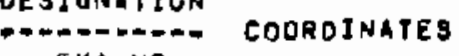

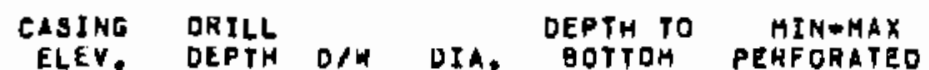

BOTTDH PEHFOAATE

DATE

COMP. FORMER

(M-Y) DESIGNATION

COMMENT\$

$41-11=0$

$41-11=9 \quad N 035253$

29942395

P. 075014

(FT) (FT) (IN)

$661,63 \quad 75$

$8.6 \quad 75$

$3=62$

$41-11 \% 22035286$

$662.09 \quad 75$

0,075

$3-62$

$41+11-3 \quad N 035253$

299 W23 97 P

563,0

75

$8.0 \quad 75$

$3-62$

41 - on 4 NO35316

$299 * 2348 \quad * 075731$

602,72

$B, 0$

15

$3-62$

01-6.6 NOS5300

$299 \times 23 \quad 99$

662.13

75

$0,8 \quad 135$

$4-13$

$01-8-7$

* 075767

$299 \times 23100$

$p^{N 035318}$

652,22

79

$0,0 \quad 79$

$3-62$

$41-8-11$
$299+43109$

N035391

661.94

79

6.075

$3 m 62$

41-8-2

+675789

$\left(\frac{1000}{10}\right.$

75

$B .0 \quad 59$

$3-82$

41- 8- 3

P 075744

662,41

79

663,20

75

$8.0 \quad 55$

$3-62$

$41-9=3$

$P^{N} 035355$

661.80

75

0.0

55

$3-82$

41-9-4 N 935317

299 He3 105

. 075833

061.56

100

$0,0 \quad 55$

$3-62$

41.9 .6

$297+23106$

N 135297

061.47

75

8.055

$3=62$ 


\begin{tabular}{|c|c|c|c|c|c|c|c|c|c|c|}
\hline $\begin{array}{l}\text { WELL } \\
\text { OESIGNATION } \\
\text { EMA ND, }\end{array}$ & CDOROINATES & $\begin{array}{c}\text { CASING } \\
\text { ELEV. } \\
\text { (FTOMSL) }\end{array}$ & $\begin{array}{l}\text { DRILL } \\
\text { DEPTH } \\
\text { [FT\} }\end{array}$ & 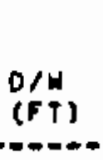 & $\begin{array}{l}\text { OIA: } \\
\text { (IN) }\end{array}$ & $\begin{array}{l}\text { DEPTH TO } \\
\text { OOTTOH } \\
\text { (TY) }\end{array}$ & $\begin{array}{l}\text { MINOMAX } \\
\text { PERFORATED } \\
\text { DEPTH EFT }\end{array}$ & $\begin{array}{l}\text { DATE } \\
\text { COMP, } \\
(M-Y) \\
-O-D .\end{array}$ & $\begin{array}{l}\text { FORMER } \\
\text { DESIGNATION }\end{array}$ & COMHENTS \\
\hline $\begin{aligned} 41-9-7 \\
299 \text { W23 } 107\end{aligned}$ & $\begin{array}{l}P_{H}^{N} 035319 \\
W 07597\end{array}$ & 661.18 & 75 & & 8,0 & 59 & & $3-62$ & & \\
\hline $\begin{aligned} 41 * 9 * 9 \\
290\end{aligned}$ & $\begin{array}{l}P^{N} 035368 \\
\\
W 75915\end{array}$ & 661.13 & 130 & & 8.6 & 130 & & $3-62$ & & DEEPENED $3=73$ \\
\hline $\begin{array}{c}41-9-11 \\
299+23169\end{array}$ & $\begin{array}{l}P^{N} 035392 \\
H 075889\end{array}$ & 361,12 & 75 & & 8,8 & 55 & & $3=62$ & & \\
\hline $\begin{array}{rll}41-9-2 & \\
299 & \text { W3 } 110\end{array}$ & $\begin{array}{l}P^{N} 035391 \\
N 075847\end{array}$ & 601,37 & 75 & & 8,0 & 55 & & $3=62$ & & \\
\hline $\begin{array}{c}41-12=2 \\
298 \text { W2 } 111\end{array}$ & $\begin{array}{l}P_{H}^{N} 035277 \\
H 015832 .\end{array}$ & 661.48 & 120 & & 8,0 & 55 & & $3-62$ & & \\
\hline $299^{9}+23112$ & $P^{N} 015824$ & 661.79 & 75 & - & 8.8 & 53 & & $3=62$ & & \\
\hline $\begin{array}{rll}41+120 & 6 \\
299+133 & 113\end{array}$ & $\begin{array}{l}P_{W 035195}^{N} 075865 \\
{ }_{N}\end{array}$ & 661,52 & 75 & & 8.0 & 55 & & $3=62$ & - & \\
\hline $\begin{array}{r}91-12=7 \\
299+23110\end{array}$ & $\begin{array}{l}P^{N} 035206 \\
N 075895\end{array}$ & 661.05 & 75 & & 8,0 & 53 & & $4=62$ & & \\
\hline $\begin{array}{rl}91-127 & 9 \\
299 & 115\end{array}$ & $P_{H}^{N 835234}$ & 661,19 & 75 & & 8.0 & 53 & & $3-62$ & & \\
\hline $\begin{array}{l}A 1-12+10 \\
299-23116\end{array}$ & $P^{N 875909}$ & 661.15 & 79 & & 8.0 & 55 & & $3=62$ & & \\
\hline 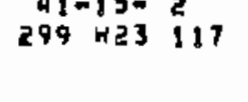 & P & 661.43 & 15 & & 8.8 & 55 & & $3-62$ & & \\
\hline $\begin{array}{c}01-15=3 \\
299 \text { H25 } 118\end{array}$ & $\begin{array}{l}P_{H}^{N} 035126 \\
H 075024\end{array}$ & 661,34 & 79 & & 8,0 & 55 & & $4=62$ & & \\
\hline
\end{tabular}




\begin{tabular}{|c|c|c|c|c|c|c|c|c|c|c|}
\hline DESIGNATIDN & COOADINATES & $\begin{array}{l}\text { CASING } \\
\text { ELEV, } \\
\text { (FTAMBL) }\end{array}$ & $\begin{array}{l}\text { ORILL } \\
\text { OEPTH } \\
\text { (FT) }\end{array}$ & $\begin{array}{l}0 / 4 \\
(F T) \\
(F)\end{array}$ & $\begin{array}{l}\text { DIA: } \\
\text { (IN) }\end{array}$ & $\begin{array}{l}\text { DEPTH TO } \\
\text { BOTTOM } \\
\text { (FT) }\end{array}$ & $\begin{array}{l}\text { MIN-HAX } \\
\text { PERFORATED } \\
\text { DEPTH (FT) }\end{array}$ & $\begin{array}{l}\text { OATE } \\
\text { COMP. } \\
(M-Y)\end{array}$ & $\begin{array}{l}\text { FORMER } \\
\text { DESIGNATION }\end{array}$ & COMMENTS \\
\hline $\begin{array}{l}41-15+5 \\
299 \times 23119\end{array}$ & $\begin{array}{l}P^{N} 635098 \\
W 075854\end{array}$ & 681.27 & 75 & & 8.0 & 55 & & $3=62$ & & \\
\hline 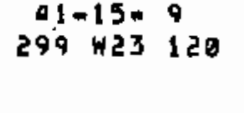 & $\begin{array}{l}P_{W}^{N} 035134 \\
W^{2}\end{array}$ & 661,04 & 15 & & 0,0 & 55 & & $4-62$ & & \\
\hline $\begin{array}{c}91-15=10 \\
299 \text { W2? } 121\end{array}$ & $\begin{array}{l}P^{N} 035177 \\
N 075904\end{array}$ & 660.90 & 129 & & 8,0 & 125 & & $4=62$ & & OEEPENED $\quad 4-73$ \\
\hline 290 w23 122 & 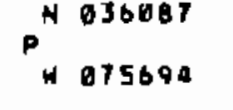 & 665.51 & 100 & & 6.0 & & & $5-70$ & $241-s-1$ & \\
\hline 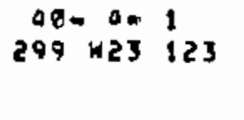 & $\begin{array}{l}P_{N 036161}^{N 075636}\end{array}$ & $665.8 \%$ & 100 & & 6,5 & & & 5.70 & $241-5-2$ & \\
\hline $\begin{array}{rll}904 & 9= & 5 \\
290 & 123 & 124\end{array}$ & $\begin{array}{l}P_{N 036090}^{N 075631} \\
N 00\end{array}$ & 605.81 & 100 & & 6.0 & & & $5-70$ & $241-5-3$ & • \\
\hline $\begin{aligned} 91-5 * 5 \\
299+123123\end{aligned}$ & $\begin{array}{l}p^{N 035405} \\
H 075750\end{array}$ & 602,12 & 123 & & 6,6 & 135 & & $a-73$ & & DEEPENED AnT3 \\
\hline $\begin{aligned} 91-507 \\
299^{5}=23 \\
126\end{aligned}$ & $\begin{array}{l}D^{N 035410} \\
W 130792\end{array}$ & 662.73 & 130 & & 6,0 & 125 & & $8=0$ & & $\begin{array}{l}\text { DEEPENEO AND GROUTED } \\
8.78\end{array}$ \\
\hline $\begin{array}{rl}91-5 * & 8 \\
899+123 & 127\end{array}$ & $\begin{array}{l}P_{N 035449}^{N 075817} \\
N\end{array}$ & 661.92 & 130 & & 6,0 & 125 & & $0=0$ & & $\begin{array}{c}\text { DEEPENED AND GROUTEO } \\
B=70\end{array}$ \\
\hline $\begin{array}{c}41-5 \times 10 \\
299 \text { w23 } 128\end{array}$ & $\begin{array}{l}\rho^{N} 035473 \\
N 015409\end{array}$ & 662,23 & 130 & & 6,0 & 125 & & $a=0$ & & $\begin{array}{c}\text { DEEPENED ANO GROUTED } \\
9-78\end{array}$ \\
\hline $\begin{array}{l}4105=12 \\
299 \text { w3 } 129\end{array}$ & $\begin{array}{l}P^{N} 035488 \\
N 075760\end{array}$ & 663,28 & 130 & & 6.0 & 125 & & Bn 0 & & $\begin{array}{c}\text { DEEPENED AND GROUTED } \\
9 \rightarrow Y \mathrm{Y}\end{array}$ \\
\hline $\begin{array}{rl}41-50 & 2 \\
299 & +2313 \theta\end{array}$ & $P^{N 035475}$ & 663,00 & 130 & & 6.0 & 125 & & $0 \cdot 0$ & & $\begin{array}{c}\text { DEEPENEO AND GROUTED } \\
9-78\end{array}$ \\
\hline
\end{tabular}




\begin{tabular}{|c|c|c|c|c|c|c|c|c|c|c|}
\hline $\begin{array}{l}\text { WELL } \\
\text { DESIGNATION } \\
\text { EMA NO. }\end{array}$ & COORDINATES & $\begin{array}{c}\text { CASING } \\
\text { ELEY. } \\
\text { (FTMMSL) }\end{array}$ & $\begin{array}{l}\text { DRILL } \\
\text { DEPTH } \\
\text { (FT) }\end{array}$ & $\begin{array}{l}\mathrm{D} / \mathrm{H} \\
(\mathrm{FT})\end{array}$ & $\begin{array}{l}\text { DIA, } \\
\text { (IN) }\end{array}$ & $\begin{array}{l}\text { DEPTH TO } \\
\text { BOTTOH } \\
\text { (FT) }\end{array}$ & $\begin{array}{l}\text { MINTMAX } \\
\text { PERFORATED } \\
\text { DEPTH (FT) }\end{array}$ & $\begin{array}{l}\text { DATE } \\
\text { COMP, } \\
(M-Y)\end{array}$ & $\begin{array}{l}\text { FORMER } \\
\text { DESIGNATION }\end{array}$ & COMAENTS \\
\hline 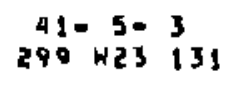 & $\begin{array}{l}P_{H}^{N 035440} \\
H 075720\end{array}$ & 662.76 & 130 & & 6,0 & 125 & & 0.0 & & $\begin{array}{c}\text { DEEPENEO AND GROUTED } \\
9.78\end{array}$ \\
\hline $\begin{array}{rl}41 * 1 * & 1 \\
299+423 & 132\end{array}$ & $\begin{array}{l}P_{N}^{N} 035590 \\
N 643\end{array}$ & 662,90 & 140 & & 6,0 & 190 & & 5.73 & & DEEPENED $5-73$ \\
\hline $\begin{array}{l}41-106 \\
299 \times 23133\end{array}$ & $\begin{array}{l}P_{N}^{N} 035508 \\
N 075665\end{array}$ & 662.92 & 100 & & 6,0 & & & $2+71$ & & \\
\hline $\begin{array}{rll}41-1 * & 8 \\
299 & 23 & 130\end{array}$ & $\begin{array}{l}P^{N} 035534 \\
n 075705\end{array}$ & 602,95 & 100 & & 6.0 & & & $1-72$ & & \\
\hline 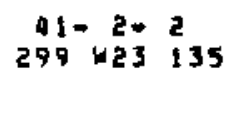 & $\begin{array}{l}P_{W}^{N} 035590 \\
W 075745\end{array}$ & 661,89 & 140 & & 6.0 & 190 & & $5-73$ & & DEEPENEO $5-73$ \\
\hline 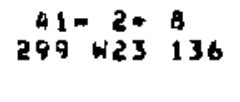 & $\begin{array}{l}P_{N 075811}^{N} 03548 \\
07581\end{array}$ & 661,64 & 100 & & 6.0 & & & $d-72$ & & \\
\hline $\begin{array}{c}41-3=12 \\
299+123137\end{array}$ & $\begin{array}{l}P^{N} 035596 \\
H 075867\end{array}$ & 661,96 & 109 & & 6,0 & 140 & & 5.73 & & DEEPENED 5.73 \\
\hline 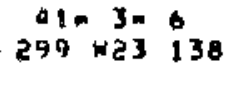 & $\begin{array}{l}P_{N}^{N} 035508 \\
N 075069\end{array}$ & 661.04 & 100 & & 6,0 & & & $2 * 72$ & & \\
\hline $299+133139$ & $\begin{array}{l}P^{N} 035548 \\
W 075913\end{array}$ & 661,28 & 100 & & 6.0 & & & $2-12$ & & \\
\hline $\begin{array}{l}41-4+1 \\
299+23100\end{array}$ & $\begin{array}{l}\text { N035488 } \\
N 075641\end{array}$ & 663.85 & 100 & & 6.8 & & & $3-72$ & & \\
\hline $\begin{array}{l}41 \times 40-11 \\
299 \times 23104\end{array}$ & $\begin{array}{l}P^{N} 035463 \\
N 015089\end{array}$ & 662.82 & 100 & & 0,0 & & & $3-72$ & & \\
\hline $\begin{array}{rl}41060 & 2 \\
289 & =23142\end{array}$ & $\begin{array}{l}P_{W 035488}^{N} 07504 \\
H\end{array}$ & 661,94 & 100 & & 6,0 & & & $2-72$ & & \\
\hline
\end{tabular}


RHO FILE
OESIGVITION
WELL
EMINO. CODROINATES

$41 \%$ 6. $5 \quad N 035419$

$41+605$
$299+23143 \quad P^{N 035419}$

$41.009 \quad N 035446$

299 W2 $144 \quad P$ W 075913

$00-1=1 \quad N 036266$

249 W23 $145 \quad P \quad 075642$

665.53

CASING DAILL

ELEY. DEPTH D/W OIA OEPTH TOTTOM PINAMAX

(FTMMSL) (FT) (FT) (IN) OTY) PT) OEPTH (FT)
(FT)

DATE

COMP, FORMER

$(\mathrm{M}=Y)$ DESIGNATION

COMMENTS

661.51

6,0

$3-73$

$661.25 \quad 199$

6.0

$3-72$

9-71

DEEPENED $\quad 5.73$

40- 1. $6 \quad N 036180$

299 W3 146 H 075605

$665,44 \quad 100$

$6,0 \quad 130$

$9=71$

40. 1. B N 036207

299 W23 147 P

$669,93 \quad 108$

6,0

6.0

$9-71$

40. 50

299 W23 148 N036266

$H 075744$

$669.19 \quad 130$

$6,0 \quad 130$

$9-71$

DEEPENED $\quad 5=73$

$40-2=1$

N $\$ 36183$

$299 \times 23149$

P

663.65

100

6,0

$9 m 71$

$40-2-10$

N 036242

$299 \times 23159$

P. 075010

665,60

100

6,0

$10-71$

108- 3. 1

N 036266

299 -23 151

P 075846

663.30

100

6,0

$10-75$

48- $3-6$

N 036183

299423152

P. 075865

$663,27 \quad 100$

6,8

$10-71$

a 8 - 3n- 9

299 H23 153

N 036242

P 075912

$662,80 \quad 130$

6,0

130

6,8

49.5.3 N 336140

$299+23 \quad 154$

$663,00 \quad 100$

$5 \cdot 7$

$16-71$

H 075724 


\begin{tabular}{|c|c|c|c|c|c|c|c|c|c|}
\hline $\begin{array}{l}\text { DESIGNATION } \\
\text { DERA NOE }\end{array}$ & CDORDINATES & $\begin{array}{l}\text { CASING } \\
\text { ELEV. } \\
\text { (FT } \rightarrow \text { HSL) }\end{array}$ & $\begin{array}{l}\text { DHILL } \\
\text { DEPTH } \\
\text { (FT) }\end{array}$ & OPI & $\begin{array}{l}\text { DIA; } \\
\{I N\}\end{array}$ & $\begin{array}{l}\text { DEPTH TO } \\
\text { BOTTOM } \\
(F T)\end{array}$ & $\begin{array}{l}\text { MIN-MAX } \\
\text { PERFORATEO } \\
\text { DEPTH (FT) }\end{array}$ & $\begin{array}{l}\text { OATE } \\
\text { COMP, } \\
\text { (MAY) }\end{array}$ & $\begin{array}{l}\text { FORMER } \\
\text { DESIGNATION COMHENTS }\end{array}$ \\
\hline $\begin{array}{rl}99-5 * & 7 \\
299 & \text { w23 } 155\end{array}$ & $P^{N} 0360851$ & 663,90 & 100 & & 6,0 & & & $10-71$ & \\
\hline $\begin{array}{c}49-5 n-18 \\
299 \text { W3 } 156\end{array}$ & $P^{N 075810}$ & 663.59 & 100 & & 6.6 & & & $10-71$ & \\
\hline 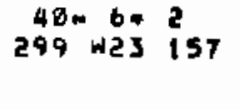 & $P^{N} 036156$ & 683.42 & 100 & & 6.0 & & & $10-71$ & \\
\hline $\begin{array}{rl}48-60 & 6 \\
299 & +23158\end{array}$ & $\begin{array}{l}N 056081 \\
N 075805\end{array}$ & 663.91 & 100 & & 6,0 & & & $16-71$ & \\
\hline $299+123159$ & $\begin{array}{l}P_{W}^{N} 0361400 \\
y^{2} 018\end{array}$ & 663.56 & 100 & & 6.0 & & & $18-71$ & \\
\hline $299^{28-73} 160$ & $P_{N}^{N} 836262$ & 665.65 & 100 & & 6,0 & & & $10=71$ & \\
\hline $\begin{array}{rll}400 & 7 n & \\
290 & 23 & 162\end{array}$ & $P_{W 075003}^{N 035972}$ & 665,46 & 100 & & 6.0 & & & $20-71$ & \\
\hline $\begin{array}{r}40-1716 \\
299+23162\end{array}$ & $P_{H}^{N} 830045785$ & 665.49 & 100 & & 6.8 & & & $18-71$ & \\
\hline 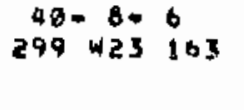 & $P^{N} 035979$ & 664.24 & 100 & & 6.0 & & & $11-71$ & \\
\hline 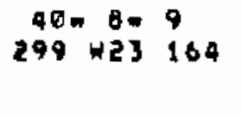 & $\begin{array}{l}N 836020 \\
F^{N} 075813\end{array}$ & 663.81 & 100 & & 6.0 & & & $10-7 \mid$ & \\
\hline $\begin{array}{rll}40-90 & 2 \\
299 & \text { w3 } & 165\end{array}$ & $\begin{array}{l}N 036059 \\
+075837\end{array}$ & 663.76 & 100 & & 6.0 & & & $10-71$ & \\
\hline $\begin{array}{rll}09 & 90 \\
289 & 63 & 166\end{array}$ & $\begin{array}{l}P_{1075877}^{N} 035477 \\
0.078\end{array}$ & 663,55 & 108 & & 6.0 & & & $10-71$ & \\
\hline
\end{tabular}




\begin{tabular}{|c|c|c|c|c|c|c|c|c|c|c|}
\hline $\begin{array}{l}\text { WELL } \\
\text { OESIGNATION }\end{array}$ & CDOROINATES & $\begin{array}{c}\text { CASING } \\
\text { ELEV. } \\
\text { (FTAHSL) }\end{array}$ & $\begin{array}{l}\text { ORILL } \\
\text { DEPTH } \\
\text { (FT) }\end{array}$ & (FT) & $\begin{array}{l}\text { oIA: } \\
\text { (IN) }\end{array}$ & $\begin{array}{l}\text { OEPTH TO } \\
\text { BOTTOH } \\
\text { (FT) }\end{array}$ & $\begin{array}{l}\text { MIN-MAX } \\
\text { PERFORATED } \\
\text { DEPTH (FT) }\end{array}$ & $\begin{array}{l}\text { DATE } \\
\text { COMP, } \\
(H \cap Y)\end{array}$ & $\begin{array}{l}\text { FORHER } \\
\text { DESIGNATION }\end{array}$ & COHMENTS \\
\hline $\begin{array}{rll}490 & 90 & 9 \\
299 & 163 & 167\end{array}$ & $\begin{array}{l}P^{N} 636938 \\
N 075912\end{array}$ & 603.06 & 300 & & 6.0 & & & 10.71 & & DEEPENEO $5=73$ \\
\hline $\begin{array}{c}49-10 n \\
279 \times 23168\end{array}$ & $\begin{array}{l}P^{N} 035929 \\
H 075619\end{array}$ & 665,70 & 100 & & 6,0 & - & & $\begin{array}{l}11-71 \\
.\end{array}$ & & \\
\hline $\begin{aligned} 40-10= & 0 \\
299 \text { w23 } 169 & \end{aligned}$ & $\begin{array}{l}P_{W}^{N} 035870 \\
W^{2} 075665\end{array}$ & 465,38 & 145 & & 6,8 & & & $11-7 t$ & & DEEPENED $5-73$ \\
\hline 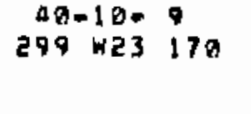 & $\begin{array}{l}P_{N}^{N} 035924 \\
N 075711\end{array}$ & 663.00 & 100 & & 6.0 & & & $11-71$ & & \\
\hline $\begin{array}{c}40-11+1 \\
299 \times 23171\end{array}$ & $\begin{array}{l}P^{N 035909} \\
H 075140\end{array}$ & 604,60 & 145 & & 6.0 & & & $\{1-7 !$ & & DEEPENED $5-73$ \\
\hline $\begin{array}{c}a 9-11=7 \\
249 \text { w2 } 172\end{array}$ & $\begin{array}{l}P^{N 035877} \\
N 075783\end{array}$ & 669.35 & 100 & & 0.0 & & & $11-11$ & . & \\
\hline $\begin{array}{c}40-11 \% 9 \\
299 \times 23173\end{array}$ & $\begin{array}{l}P^{H 035936} \\
H 075810\end{array}$ & 663,91 & 100 & & 6.0 & & & $11-71$ & & \\
\hline $\begin{array}{l}49 m+2 * 2 \\
299 \mathrm{~m} 23170\end{array}$ & $\begin{array}{l}P^{N} 035952 \\
W 075837\end{array}$ & 663.92 & 100 & & 6,0 & & & $|1+7|$ & & \\
\hline $\begin{aligned} 40+12=6 \\
299+123175\end{aligned}$ & $\begin{array}{l}P^{N 035879} \\
N 075869\end{array}$ & 663.43 & 195 & & 6.0 & & & $11-11$ & & OEEPENED $5=73$ \\
\hline 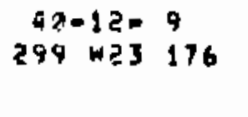 & $\begin{array}{l}P^{N} 035924 \\
W 075915\end{array}$ & 663,21 & 100 & & 6,0 & & & $11-71$ & & \\
\hline $\begin{array}{rll}40-40 & 8 \\
299 & 173 & 177\end{array}$ & $\begin{array}{l}P^{N} 036291 \\
W 075698\end{array}$ & 665,40 & & & & & & $\theta-a$ & & \\
\hline $\begin{array}{c}90-5-5 \\
299 k 23178 \\
3065\end{array}$ & $\begin{array}{l}P^{N} 43667 ? ? \\
n 075747\end{array}$ & 665,02 & 105 & & 6,0 & & & $3-76$ & & CHANGED TO WaSnIE \\
\hline
\end{tabular}




\begin{tabular}{|c|c|c|c|c|c|c|c|c|c|c|}
\hline OESIGNATION & COOROINATES & $\begin{array}{l}\text { CASING } \\
\text { ELEV } \\
\text { (ETEHSL) }\end{array}$ & $\begin{array}{l}\text { DRTLL } \\
\text { DEPTH } \\
\text { (FT) }\end{array}$ & $\begin{array}{l}0 / \% \\
(F i)\end{array}$ & DIA; & $\begin{array}{l}\text { DEPTH TO } \\
\text { BOTTOH } \\
\text { (FT) }\end{array}$ & $\begin{array}{l}\text { MINOHAX } \\
\text { PERFORATEO } \\
\text { DEPTH (FT) }\end{array}$ & $\begin{array}{l}\text { DATE } \\
\text { COMP, } \\
(M-Y)\end{array}$ & $\begin{array}{l}\text { FORMER } \\
\text { DESIGNATION }\end{array}$ & COMMENTS \\
\hline $299 \times 1=43179$ & $\begin{array}{l}\rho^{N} 036219 \\
N 015620\end{array}$ & 666,10 & 100 & & 6.8 & & & $3-74$ & & \\
\hline $\begin{array}{c}40-1-10 \\
290 \times 23180\end{array}$ & $\begin{array}{l}P_{N}^{N} 0362606 \\
N 075695\end{array}$ & 665,07 & 180 & & 6,8 & & & $3-74$ & & \\
\hline $299+25181$ & $\begin{array}{l}P^{N} 036183 \\
H 075753\end{array}$ & 664,10 & 100 & & 6,0 & & & $3-74$ & & \\
\hline $\begin{array}{l}90-2 m-11 \\
299-13982\end{array}$ & $\begin{array}{l}P^{N} 036271 \\
+875786\end{array}$ & 665,24 & 100 & & 6.0 & & & $3-74$ & & \\
\hline $\begin{array}{lll}40-30 & 30 \\
249 & 183\end{array}$ & $\begin{array}{l}\text { N } 936280 \\
{ }_{N} 075910 .\end{array}$ & 662.69 & 100 & & 6,8 & & & $3=74$ & & \\
\hline $\begin{array}{c}48-3=11 \\
299 \text { we3 } 184\end{array}$ & $\begin{array}{l}P^{N} 036271 \\
H 075689\end{array}$ & 663.63 & 100 & & 6,0 & & & $3=74$ & & \\
\hline $299+723185$ & 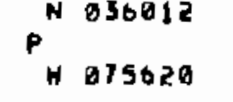 & 665,97 & 100 & & 6,0 & & & $3-74$ & - & \\
\hline 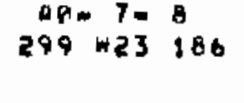 & $\begin{array}{l}\rho^{N} 036960 \\
N 075700\end{array}$ & 665.54 & 100 & & 6.8 & & & $3-70$ & & \\
\hline $\begin{array}{l}48-7=11 \\
289 \times 23187\end{array}$ & $\begin{array}{l}P^{N} 0360680 \\
N 075678\end{array}$ & 665.07 & 100 & & 6.0 & & & $9-74$ & & \\
\hline 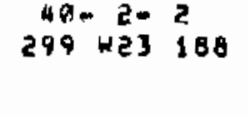 & $\begin{array}{l}P^{N} 836249 \\
N 075726\end{array}$ & 663,70 & 100 & & 6.0 & & & $2-14$ & & $\begin{array}{l}\text { ABANDONED, CASING CUT } \\
\text { OFF BELOW BRADB }\end{array}$ \\
\hline 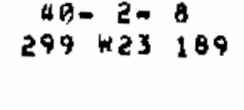 & $\begin{array}{l}p^{N 0362144} \\
W 075015\end{array}$ & 663.86 & 100 & & 0.0 & & & $2-74$ & & \\
\hline $\begin{array}{rl}41-10 & 4 \\
299 & 23 \\
190\end{array}$ & $\begin{array}{l}P^{N} 033527 \\
N 075628\end{array}$ & 661.65 & 100 & & 6,0 & & & $1107 \mathrm{a}$ & & \\
\hline
\end{tabular}




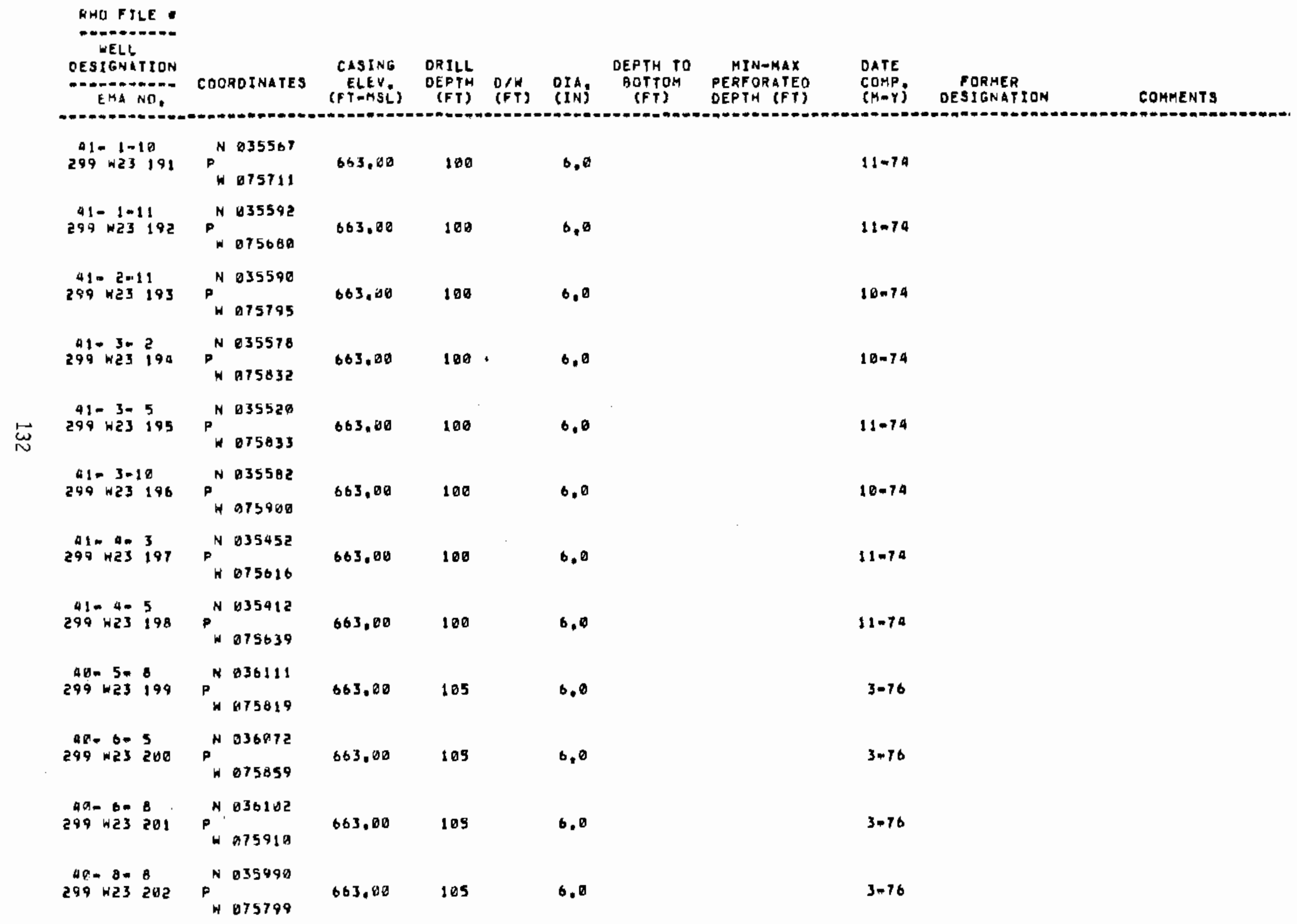




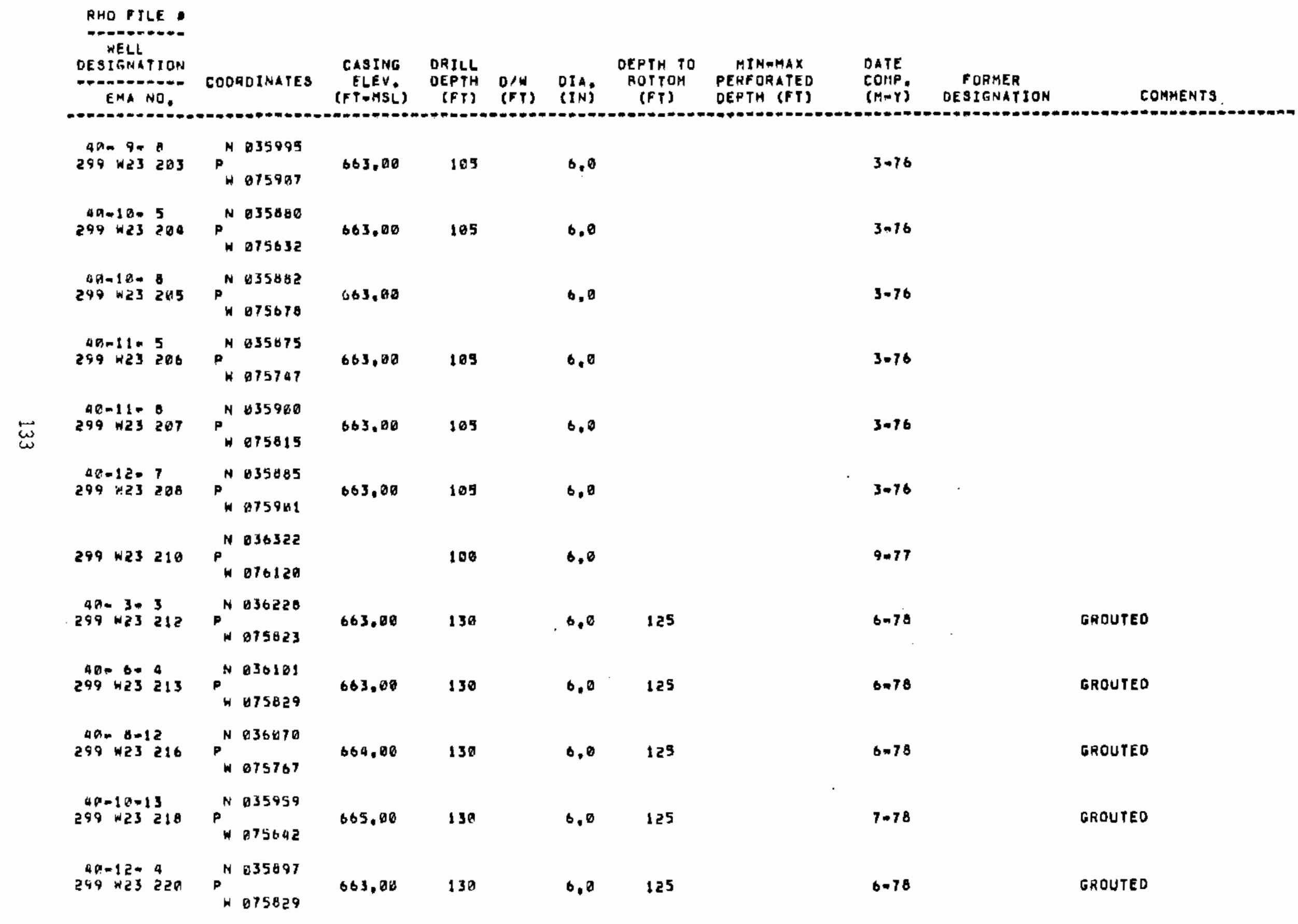




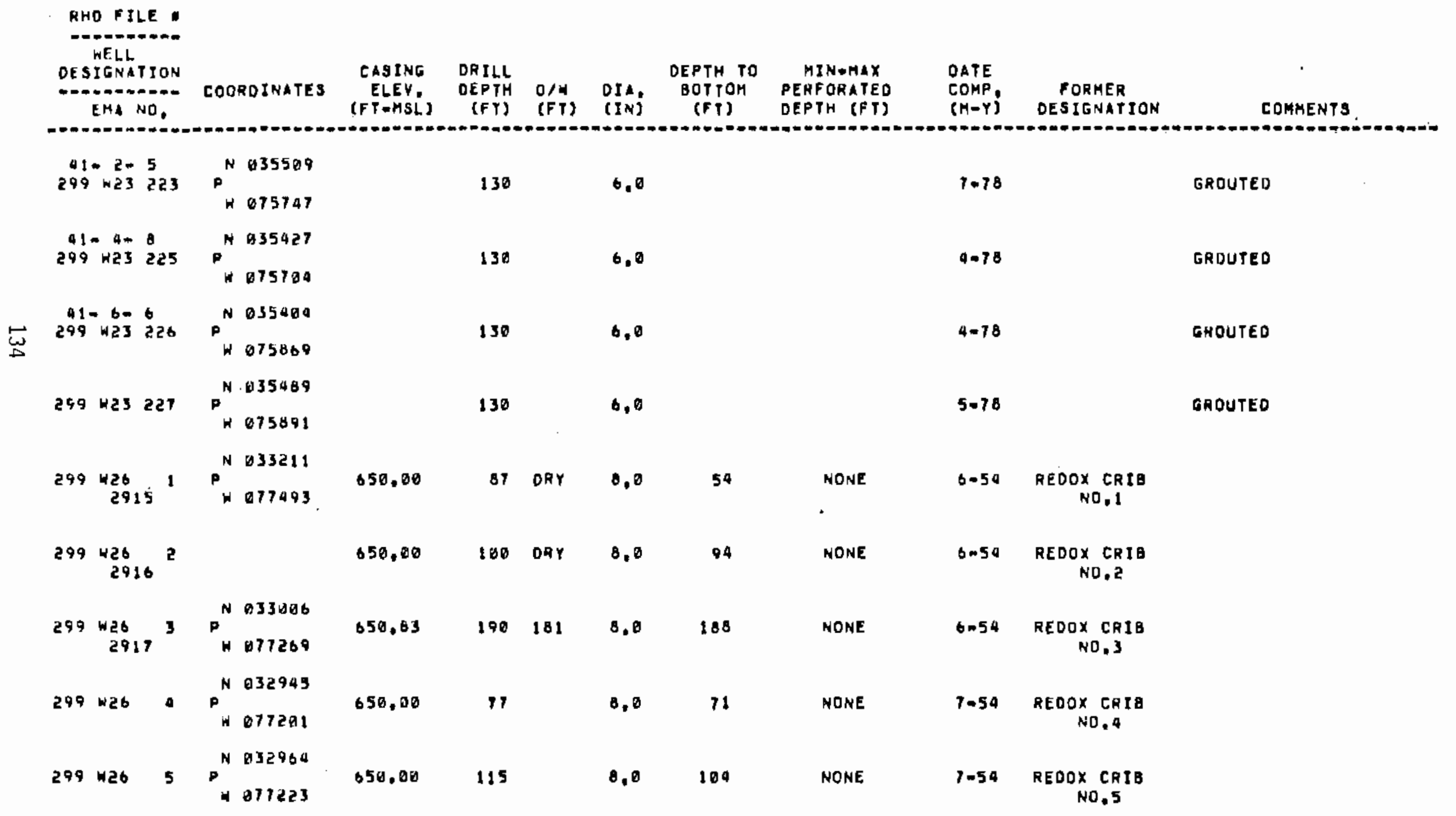




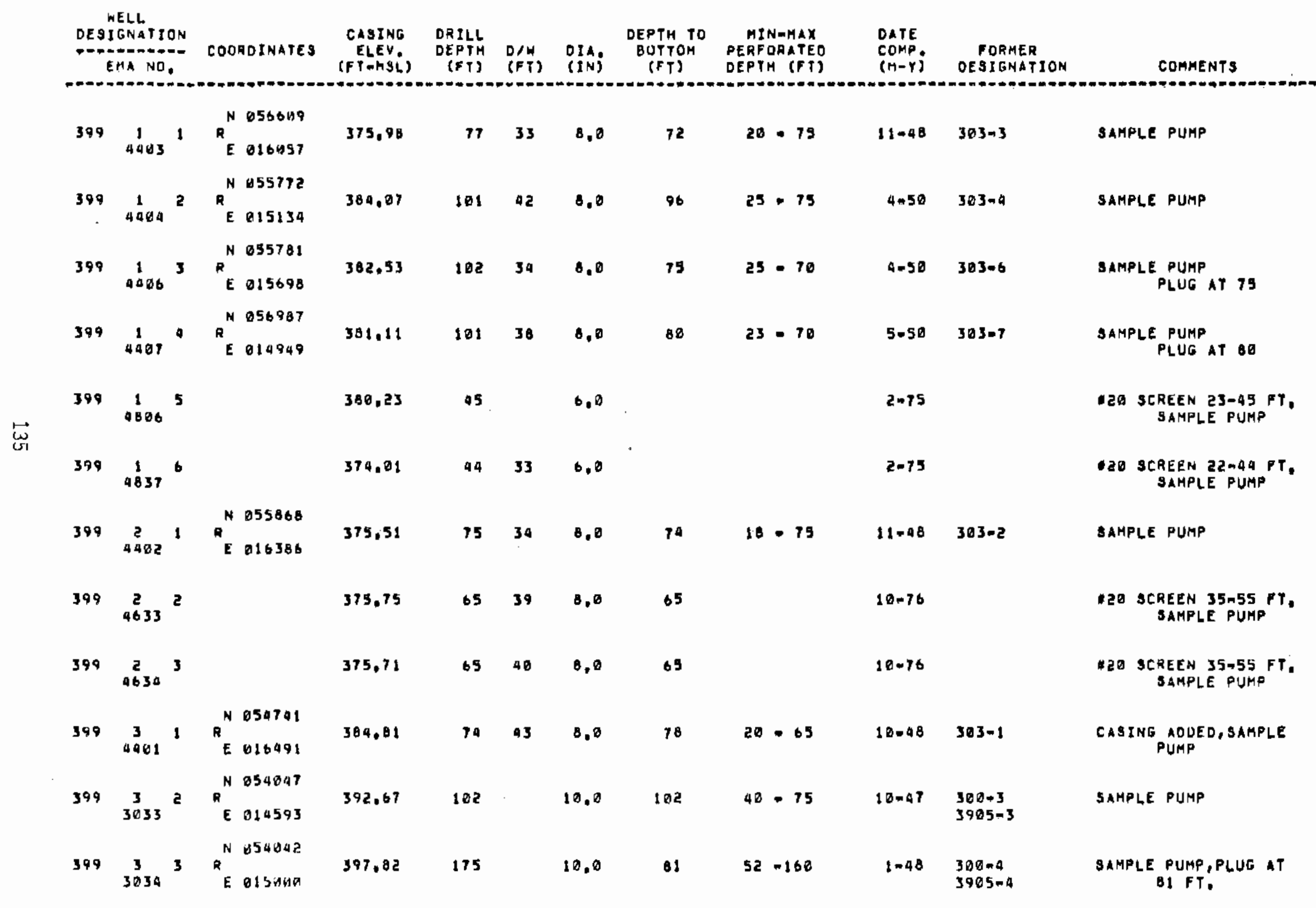




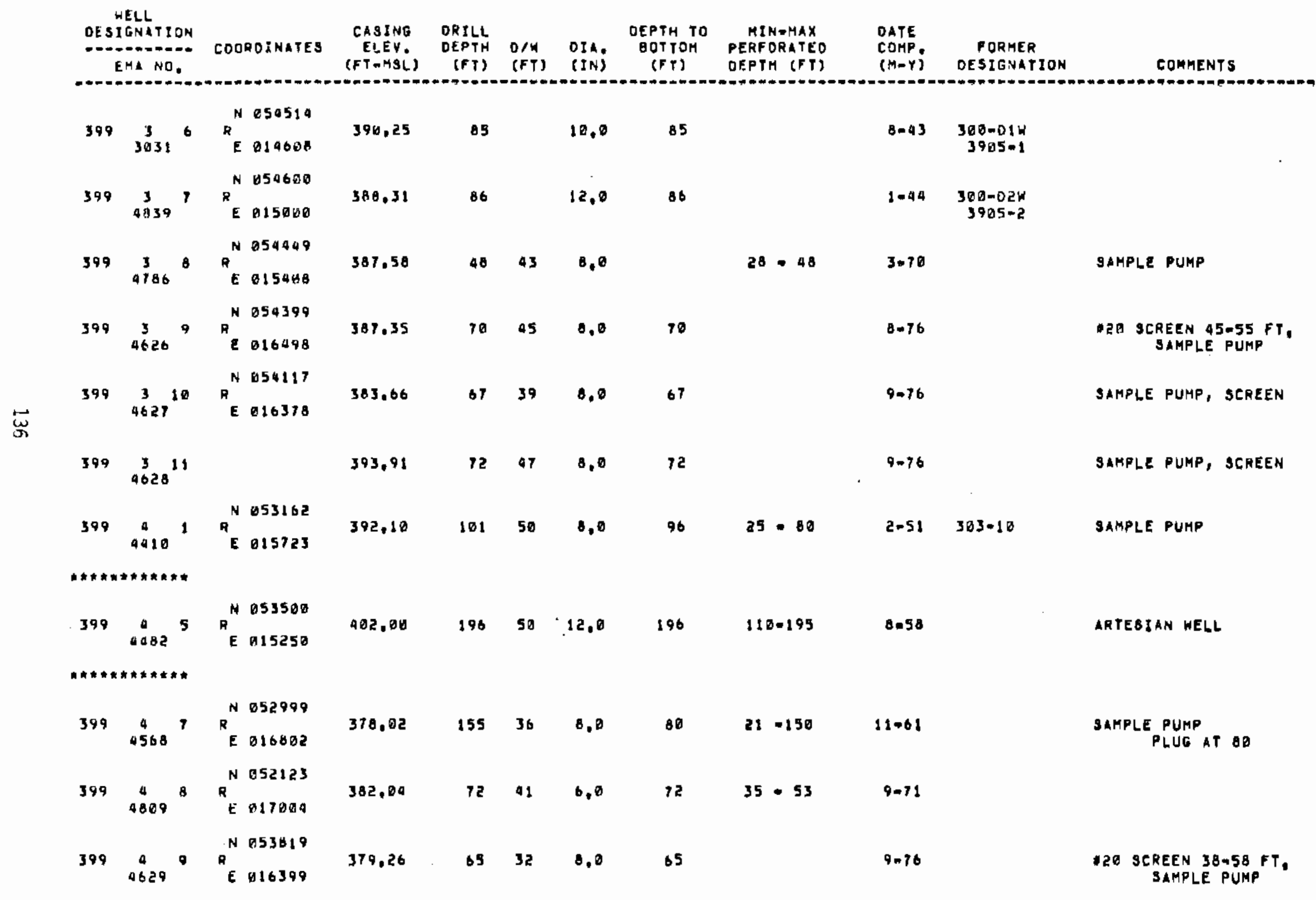




\begin{tabular}{|c|c|c|c|c|c|c|c|c|c|c|c|c|c|}
\hline $\begin{array}{r}\text { OESI } \\
\text { On }\end{array}$ & $\begin{array}{l}\text { ELL } \\
\text { GNATI } \\
\text { MA NO }\end{array}$ & & $\cos$ & RDINATES & $\begin{array}{c}\text { CASING } \\
\text { ELEV. } \\
\text { (FTAMSL) }\end{array}$ & $\begin{array}{l}\text { DRILL } \\
\text { OEPTH } \\
\text { (FT) }\end{array}$ & $\begin{array}{ll}0 / W \\
(F T)\end{array}$ & OIA & $\begin{array}{l}\text { DEPTH TO } \\
\text { BOTTOM } \\
\text { (FT) }\end{array}$ & $\begin{array}{l}\text { MINFHAX } \\
\text { PERFORATEO } \\
\text { DEPTH (FT) }\end{array}$ & $\begin{array}{l}\text { DATE } \\
\text { COMP, } \\
(\mathrm{H}=\mathrm{Y})\end{array}$ & $\begin{array}{l}\text { FORMER } \\
\text { DESIGNATION }\end{array}$ & COMHENTS \\
\hline 399 & $\begin{array}{c}4 \\
4630\end{array}$ & 10 & $a^{N}$ & $\begin{array}{l}053527 \\
016471\end{array}$ & 377.68 & $a d$ & & 0,0 & & & 9.76 & & SAMPLE PUMP, SCREEN \\
\hline 389 & 5 & 1 & $\mathrm{R}_{\mathrm{E}}^{\mathrm{N}}$ & $\begin{array}{l}053179 \\
014026\end{array}$ & 395,61 & 102 & 53 & 0.0 & 95 & $23 \cdot 100$ & $2+51$ & $303-11$ & SAMPLE PUMP \\
\hline 399 & $\stackrel{5}{4575}$ & 2 & $R_{E}^{N}$ & $\begin{array}{l}053137 \\
011393\end{array}$ & 390,75 & 424 & 9 & 0,0 & 422 & NONE & 7.59 & $303-15$ & COHFINEO AQUIFER \\
\hline \multicolumn{14}{|c|}{ 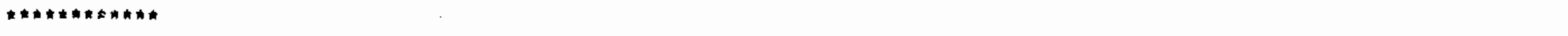 } \\
\hline 399 & 4069 & 1 & $R_{E}^{N}$ & $\begin{array}{l}054083 \\
013309\end{array}$ & 386.98 & 101 & 49 & 8,0 & 67 & $25 \cdot 75$ & $5=50$ & $363-9$ & SAMPLE PUMP \\
\hline 399 & $\stackrel{B}{4055}$ & 1 & $R_{E}^{N}$ & $\begin{array}{l}055800 \\
013657 .\end{array}$ & 399.87 & 102 & 55 & 0.0 & 98 & $35 \cdot 83$ & $4=50$ & $305-5$ & SAMPLE PUMP \\
\hline 399 & $\stackrel{8}{4408}$ & 2 & ${ }_{E}^{N}$ & $\begin{array}{l}055718 \\
012242\end{array}$ & 396,07 & 119 & $\mathbf{5 3}$ & 0.0 & 95 & $43-106$ & $5-50$ & $303-8$ & $\begin{array}{l}\text { SAMPLE PUMP, PLUG AT } \\
95 \mathrm{FT} \text {. }\end{array}$ \\
\hline 399 & 4912 & 3 & ${ }_{E}^{N}$ & $\begin{array}{l}056948 \\
013535\end{array}$ & 393.21 & 102 & 50 & 8,0 & 99 & $25=99$ & 3.51 & $383-12$ & SAMPLE PUMP \\
\hline
\end{tabular}


HELL

OESIGNHTION

COORDINATES

CASING DRILL

DEFIH TO MINAMAX

BOTTOM PERFORATEO

DATE

(FT*H3L) (FT) (FT) (IN) (FT) OEPTH (FT)

DESIGNATION

COHMENTS

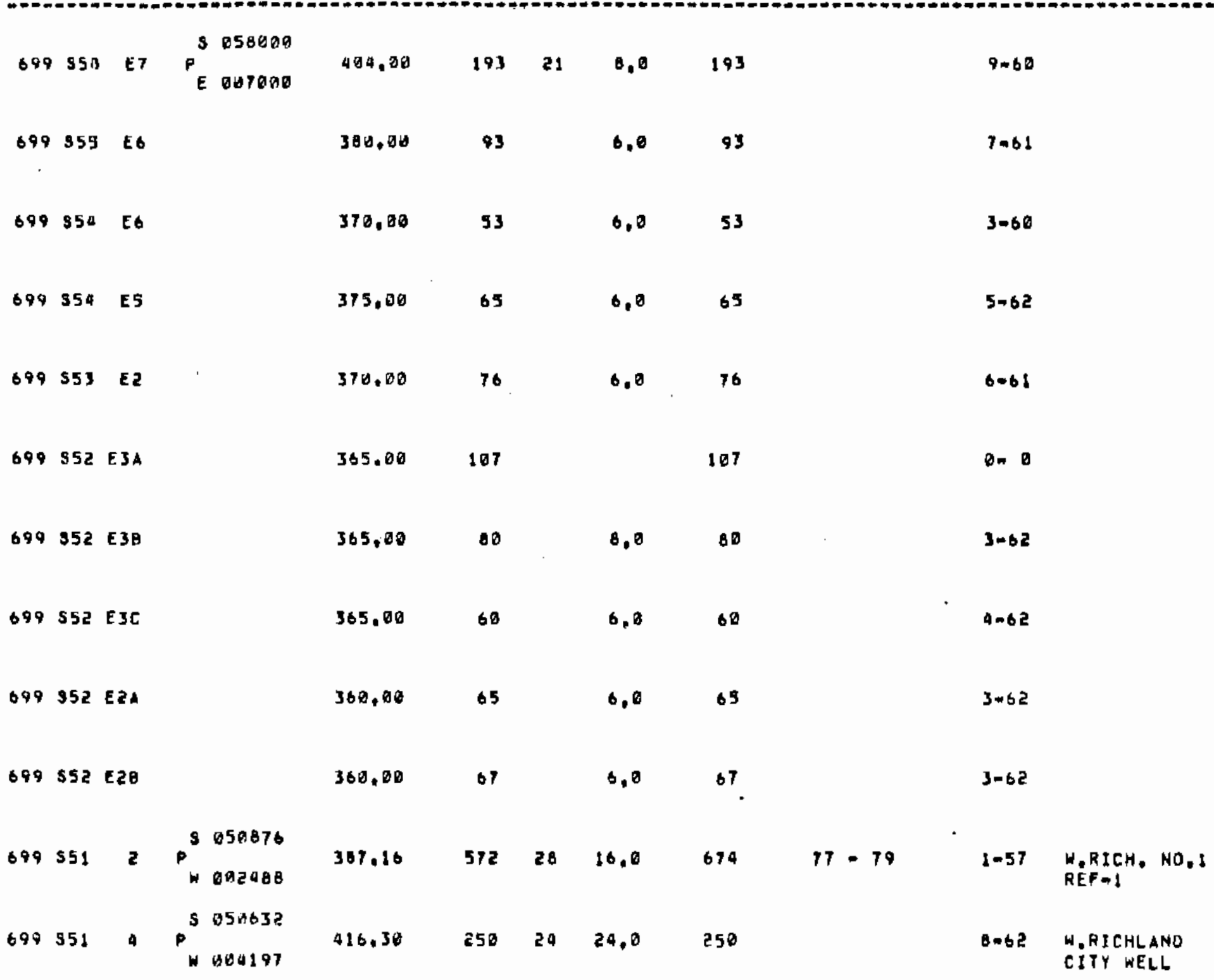




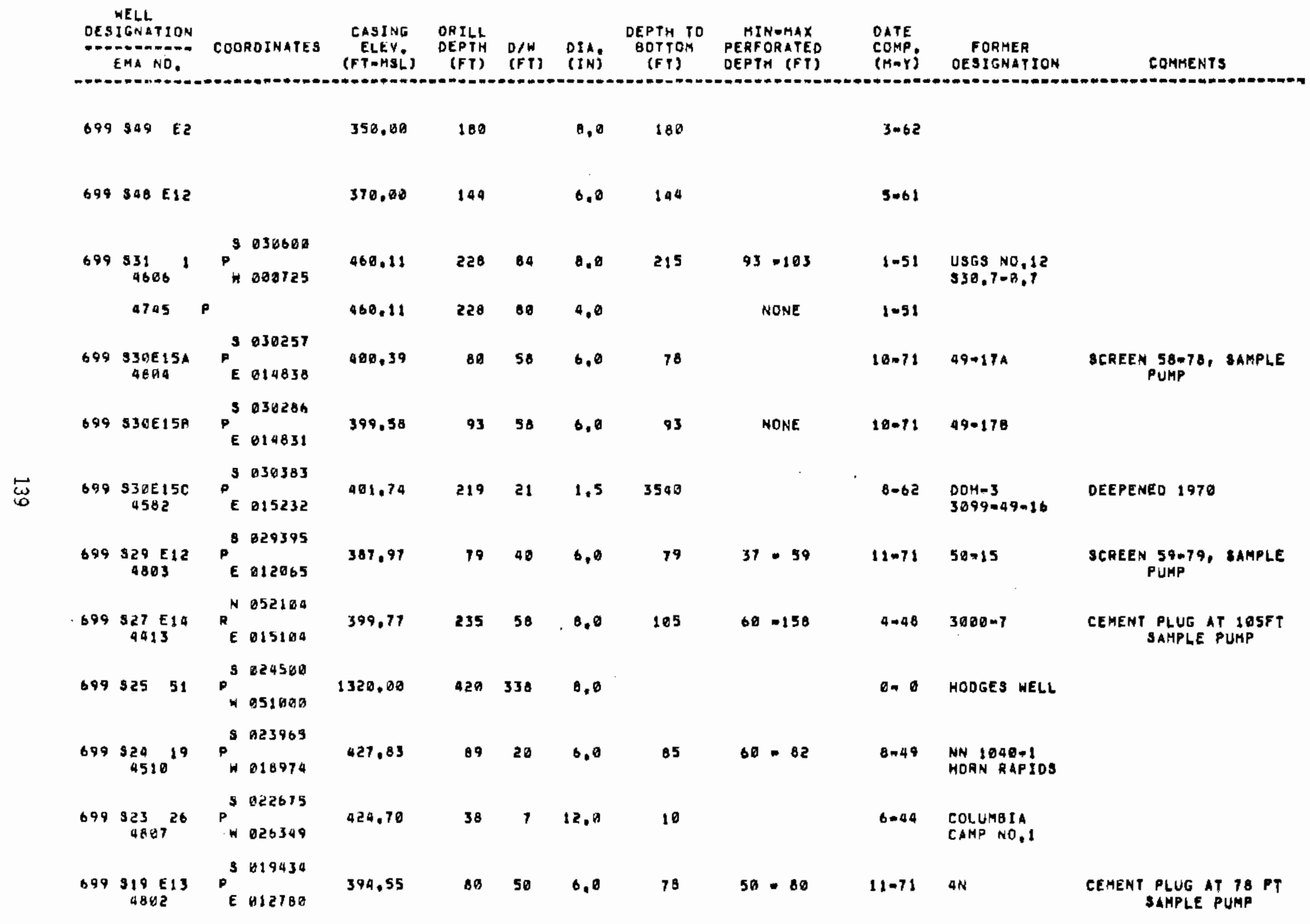




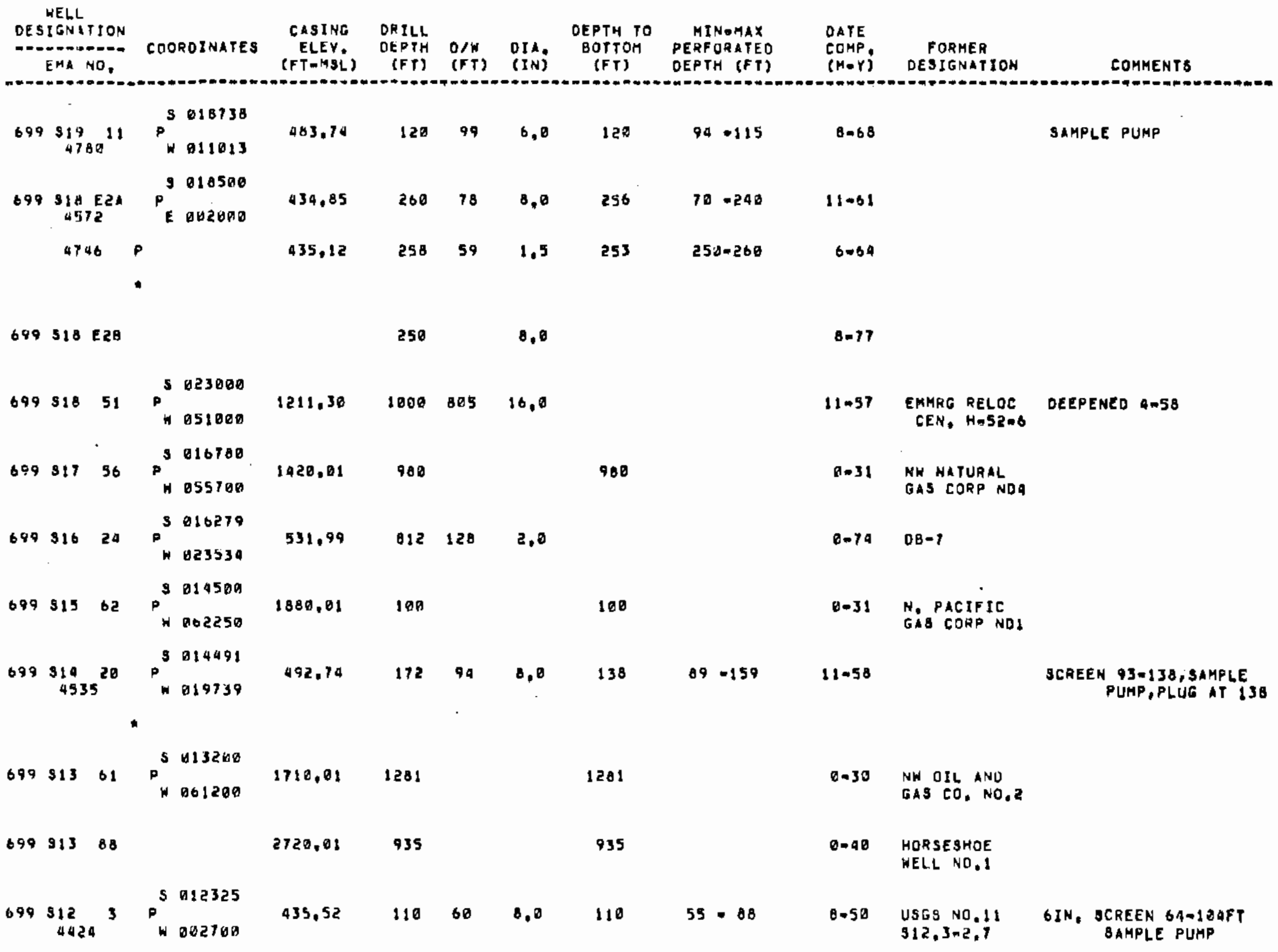




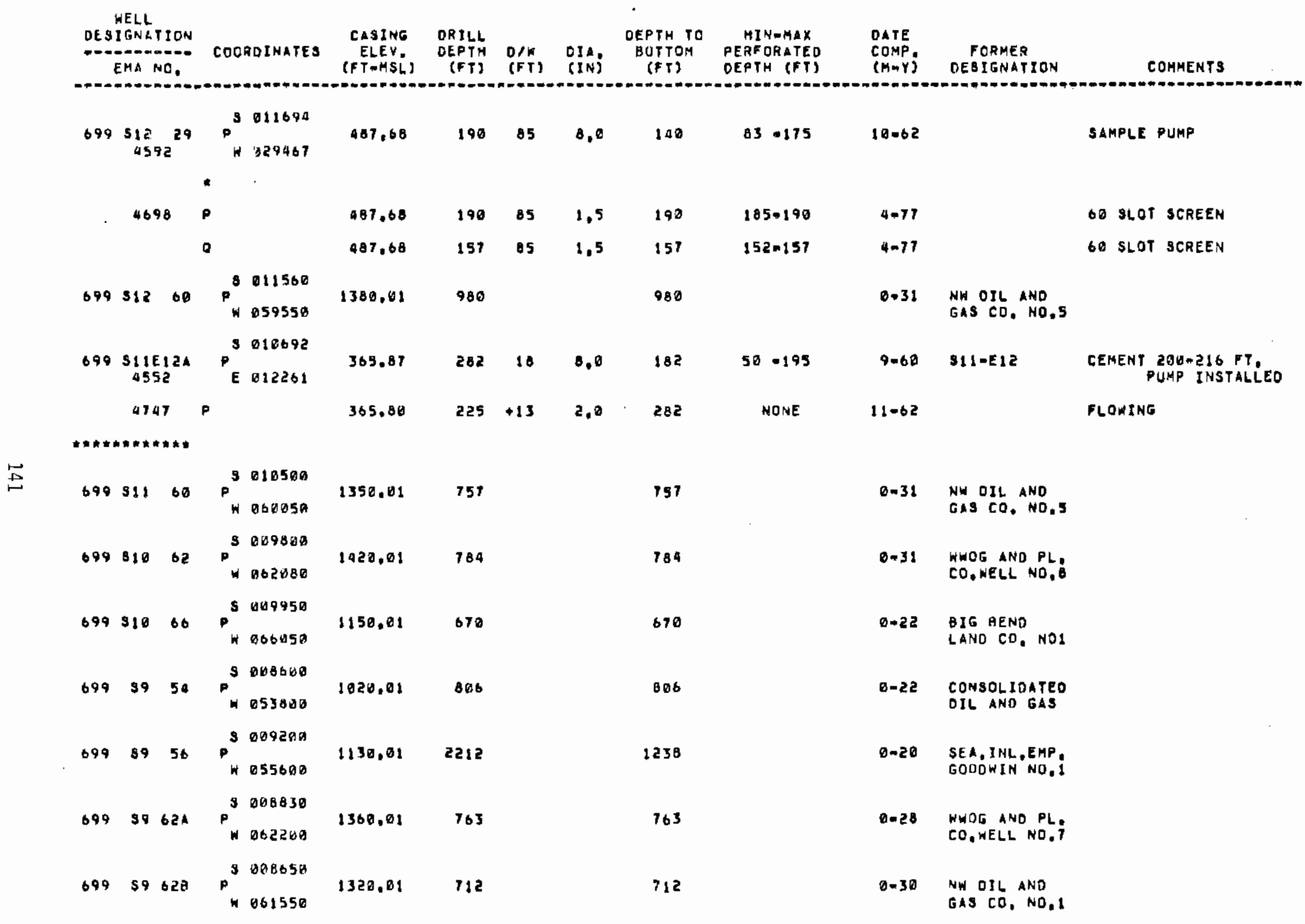




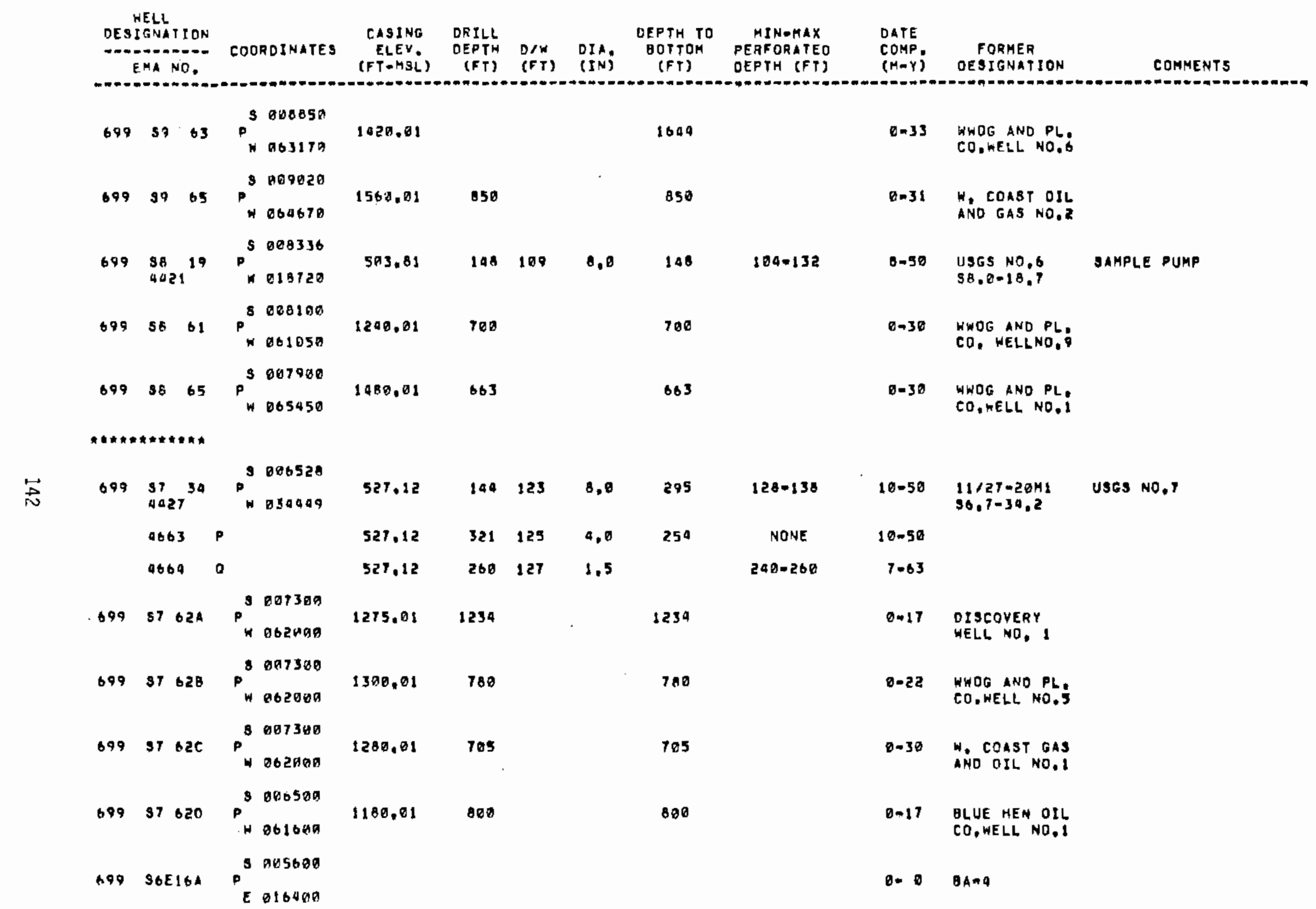




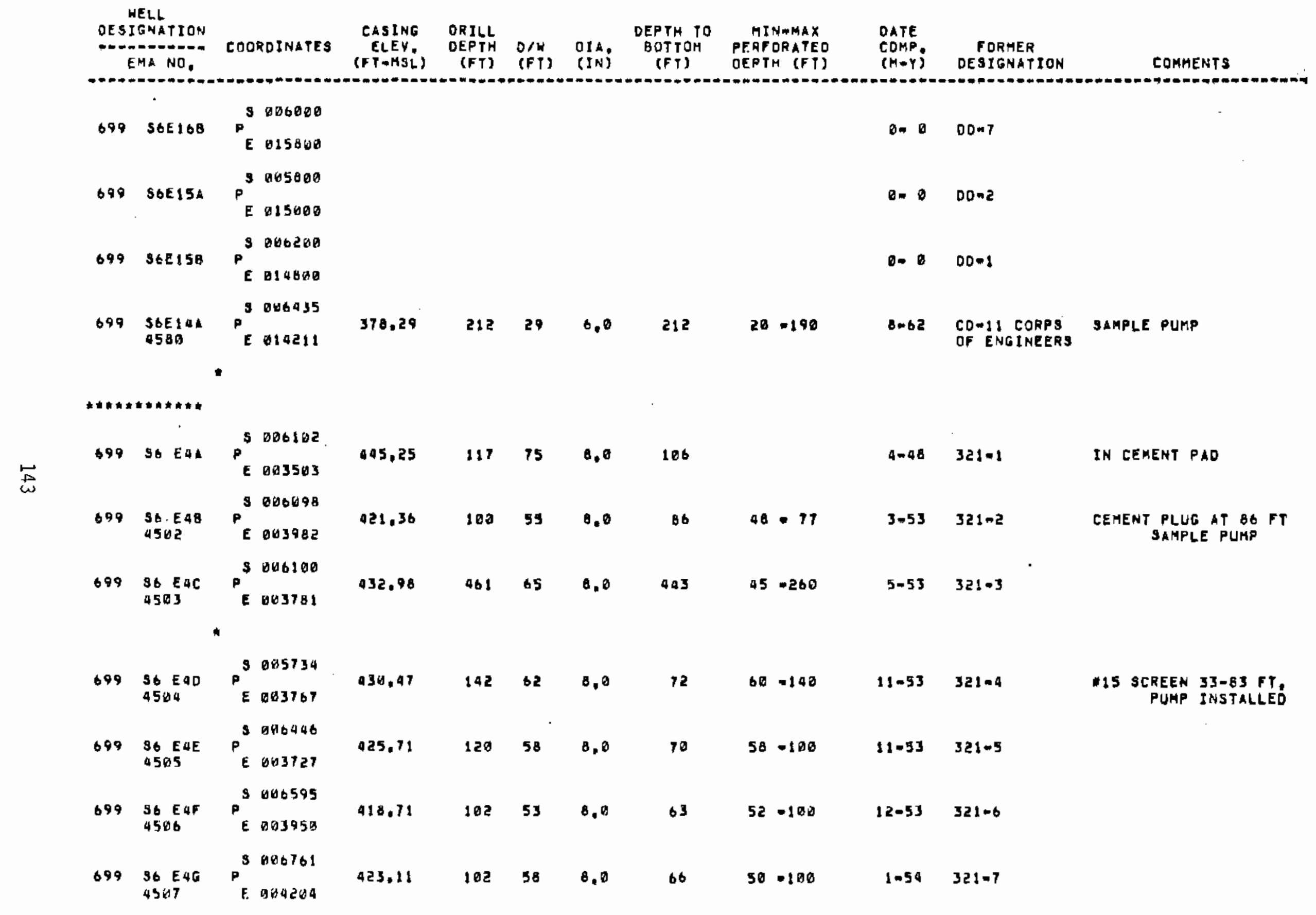




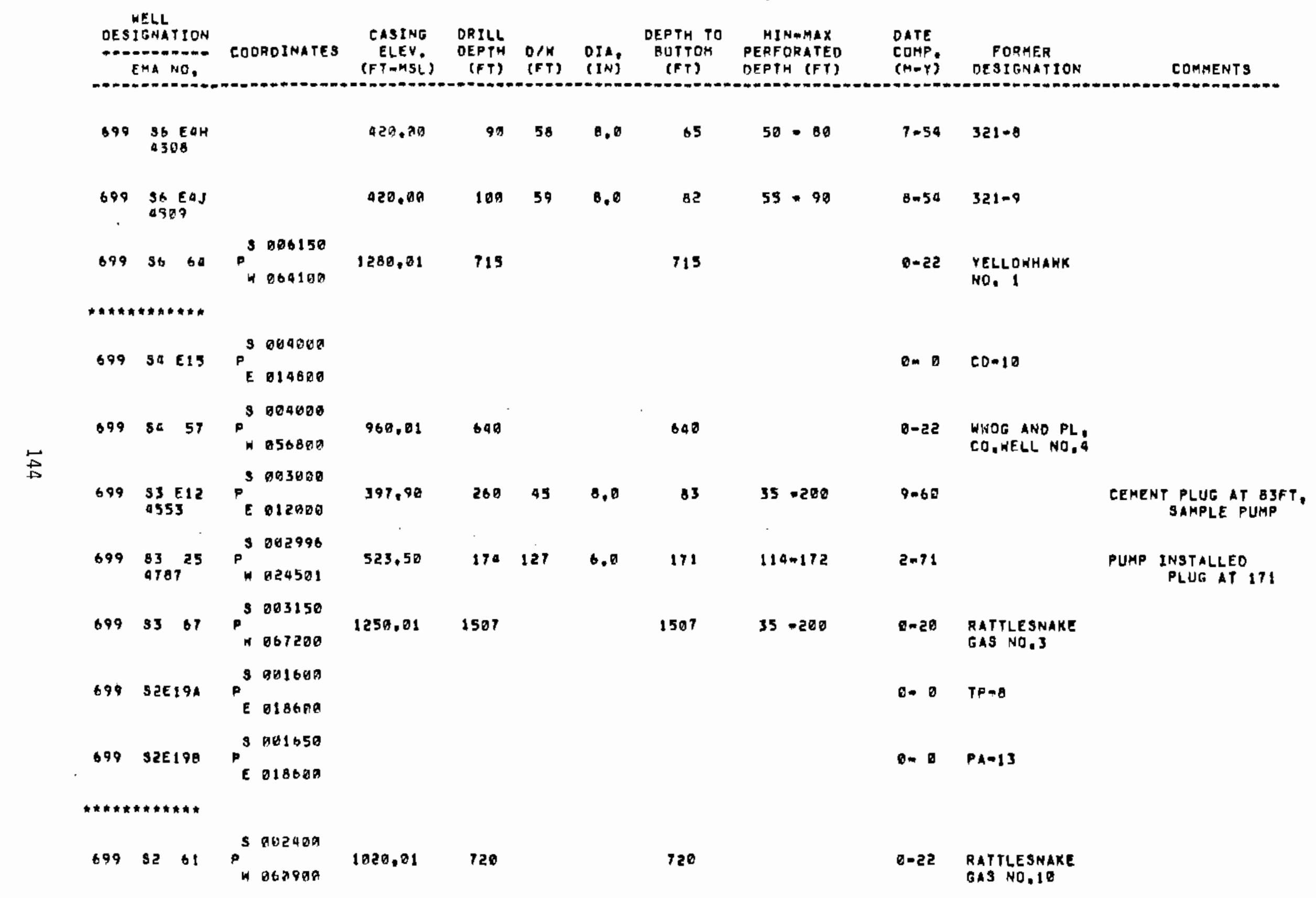




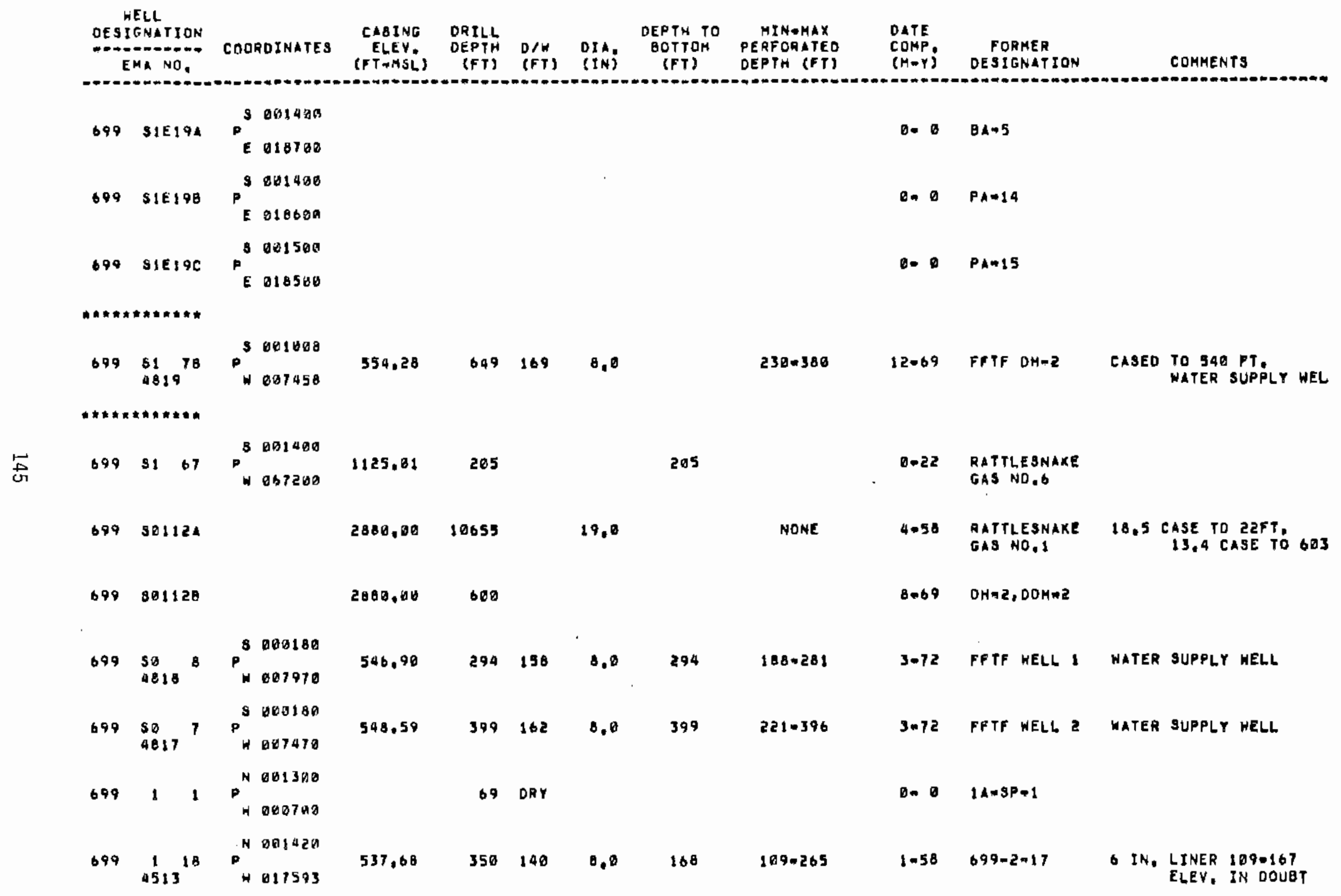




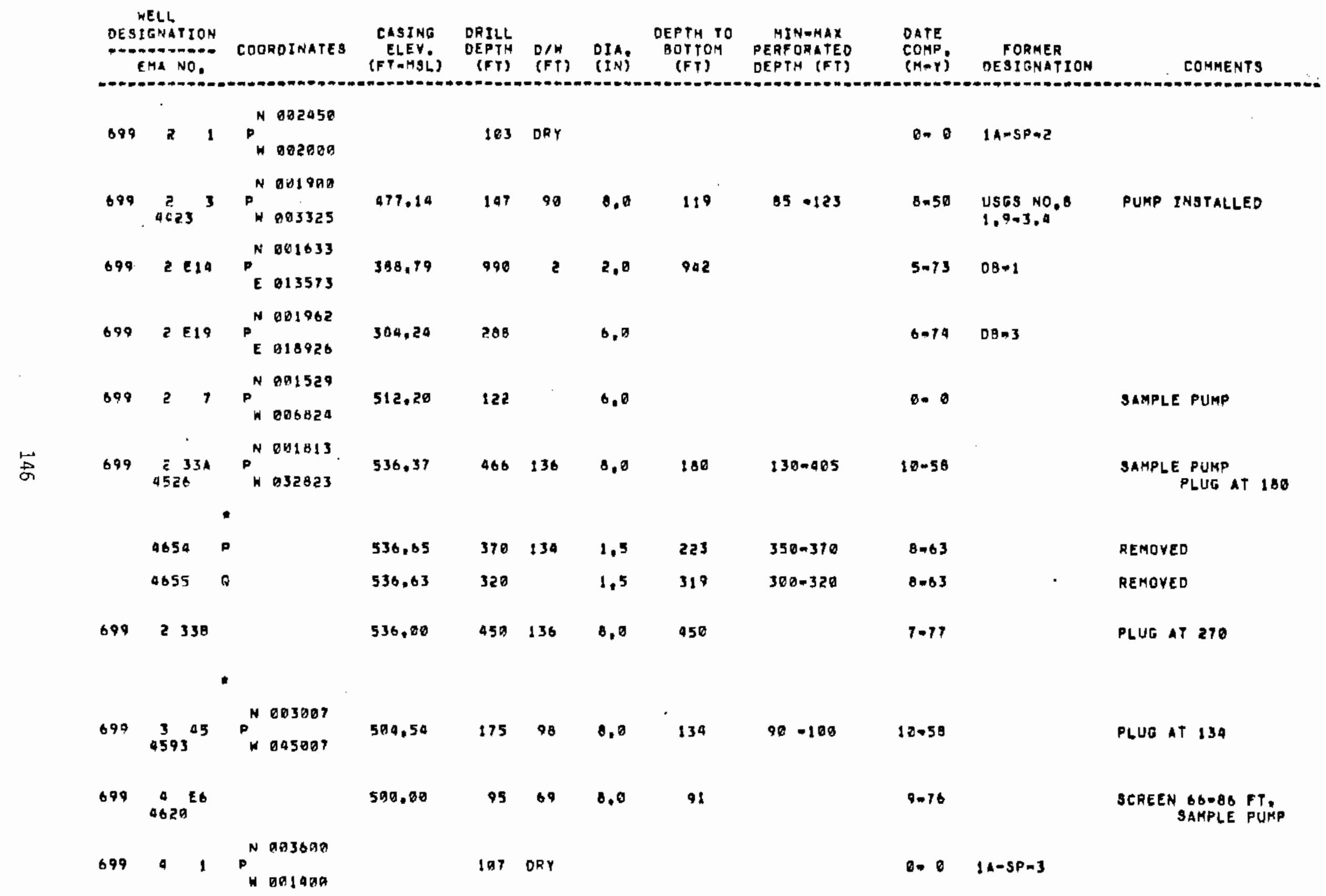




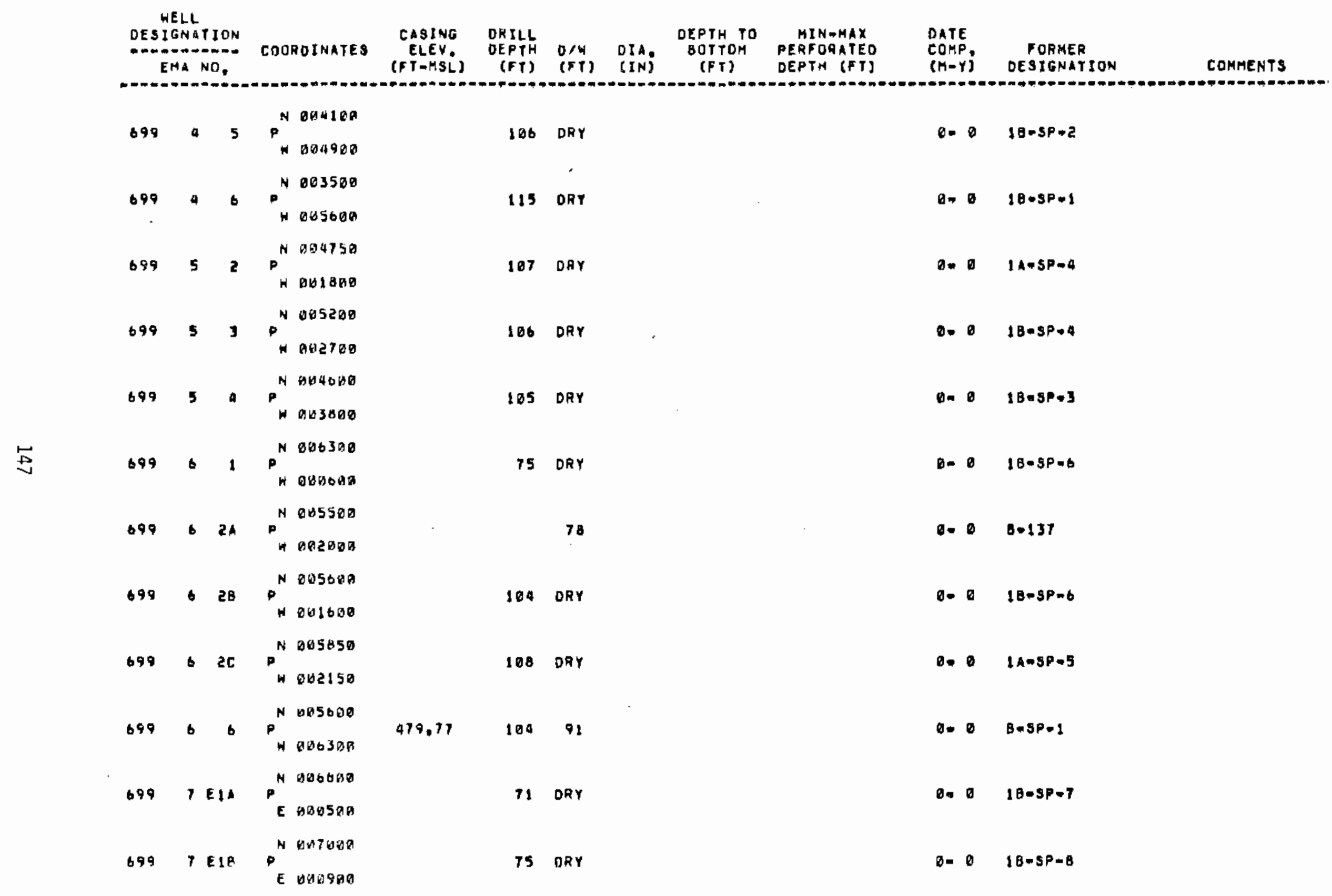




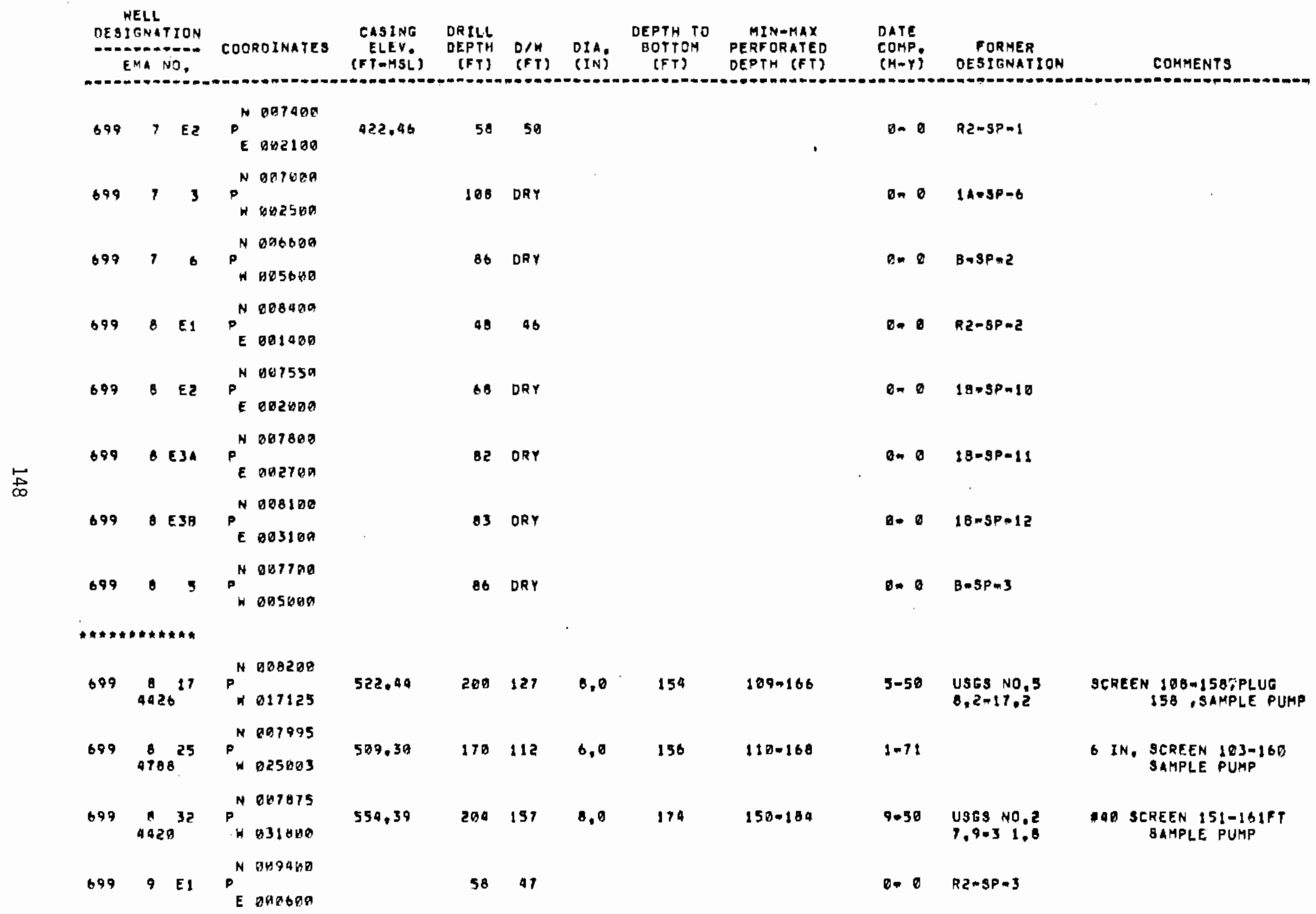




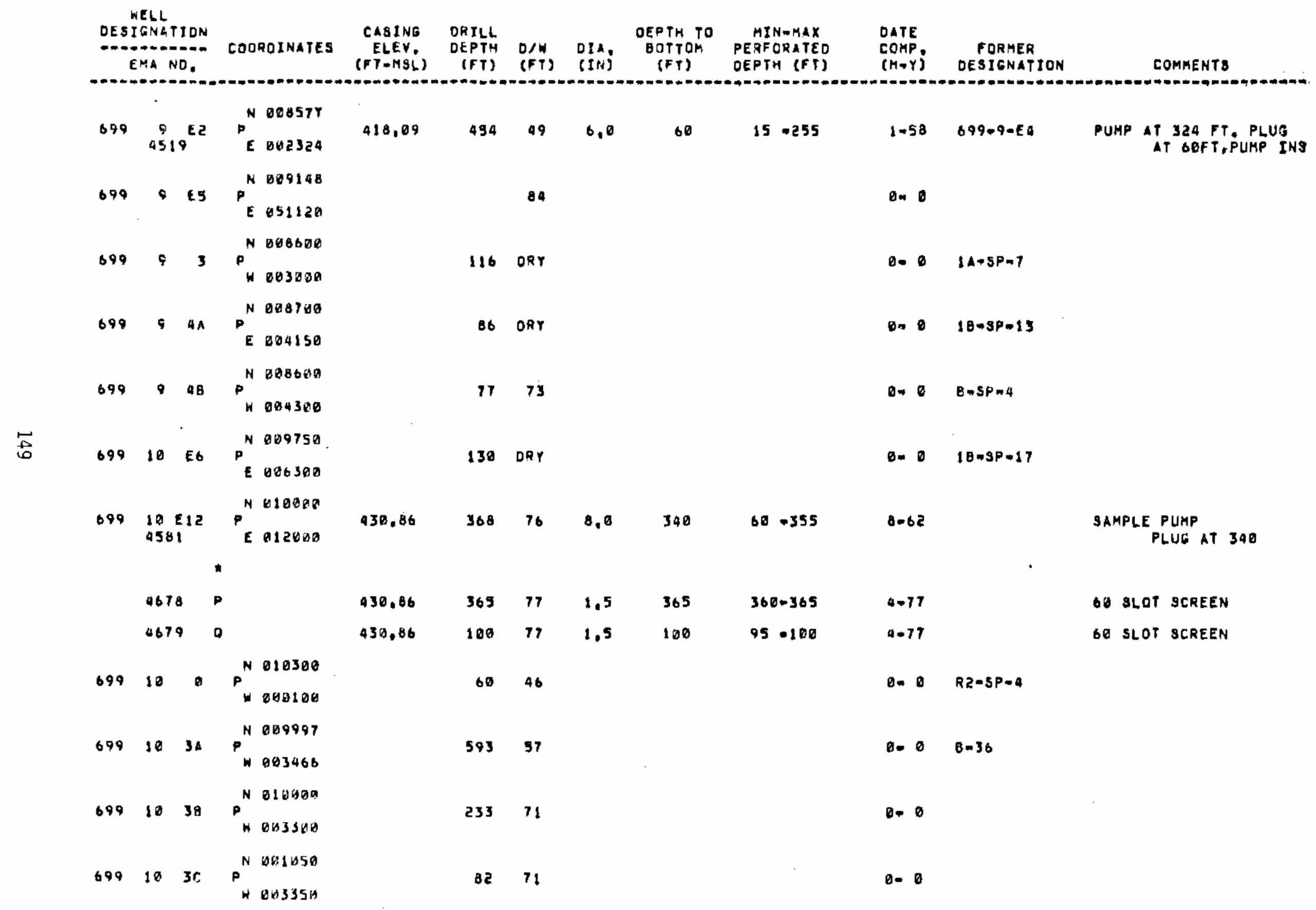


WELL

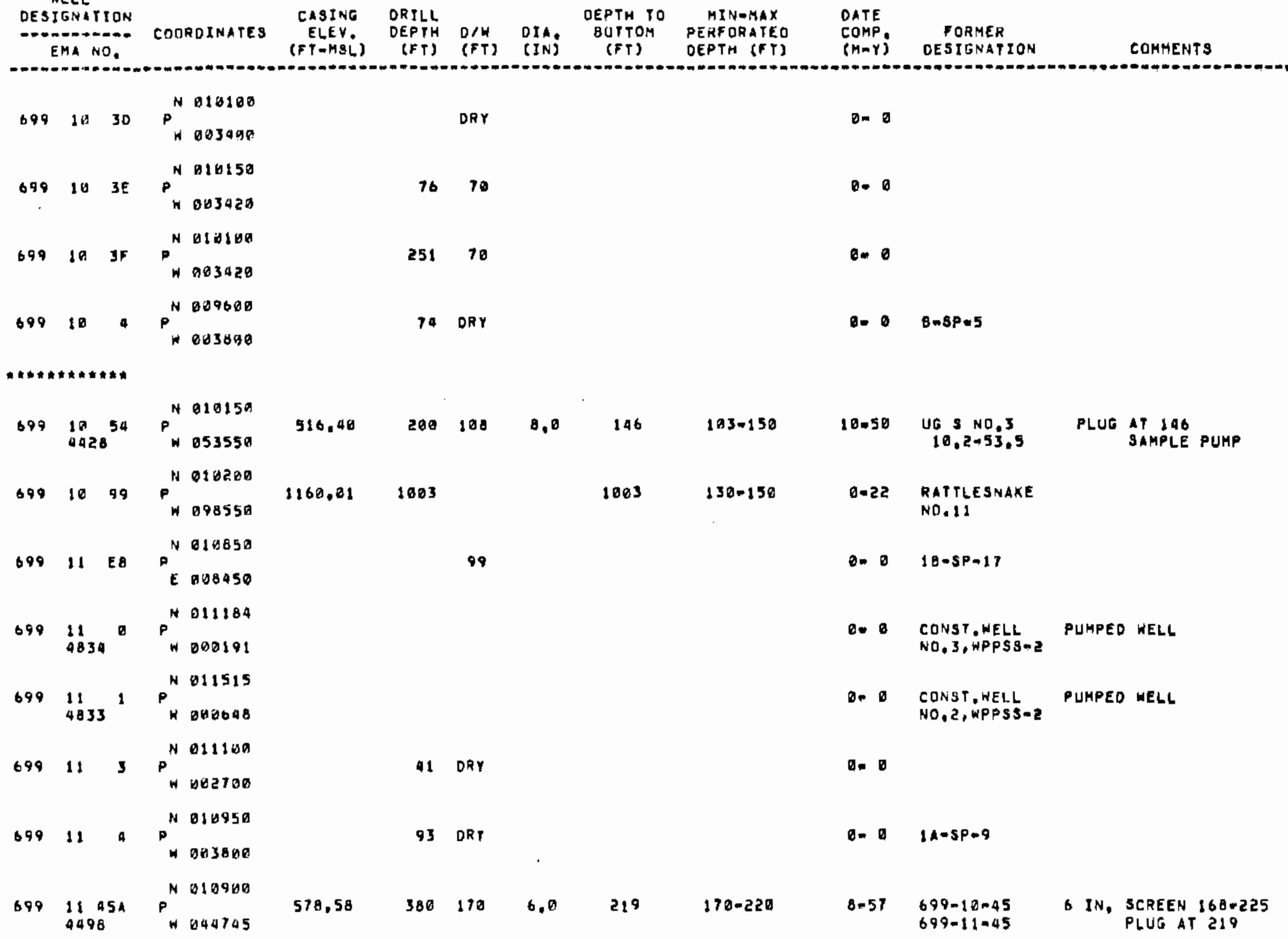




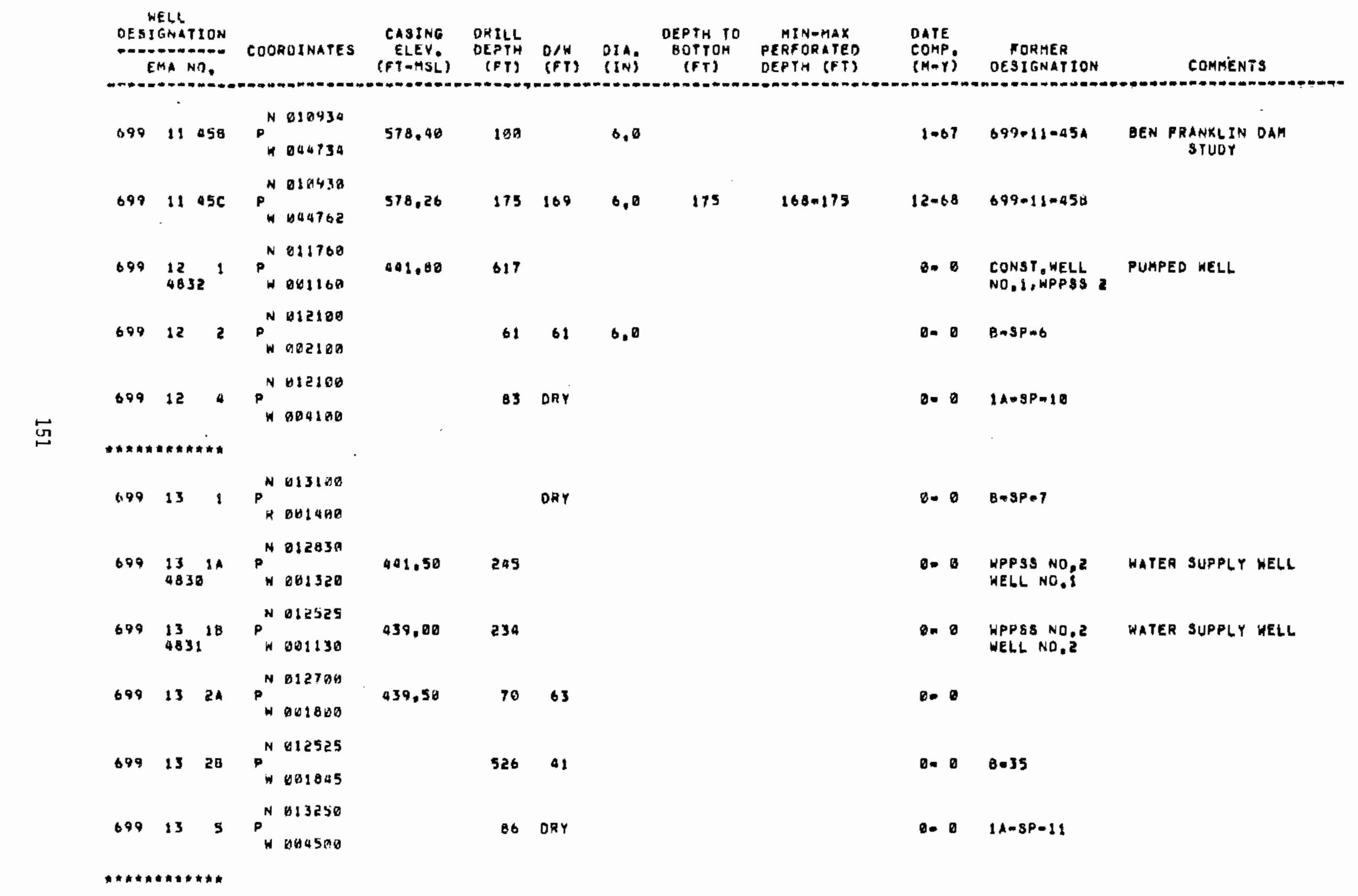




$$
\text { HELL. }
$$

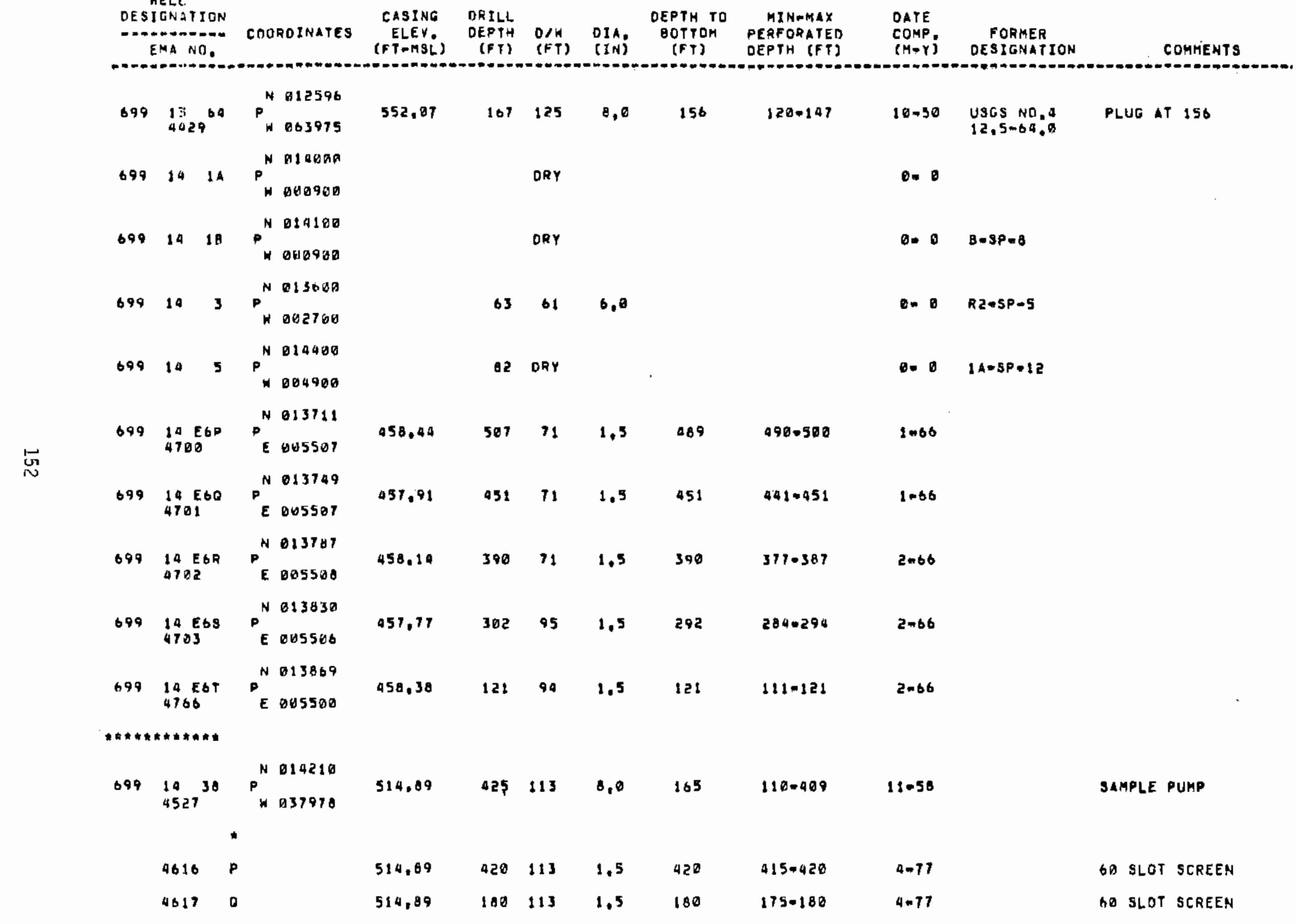




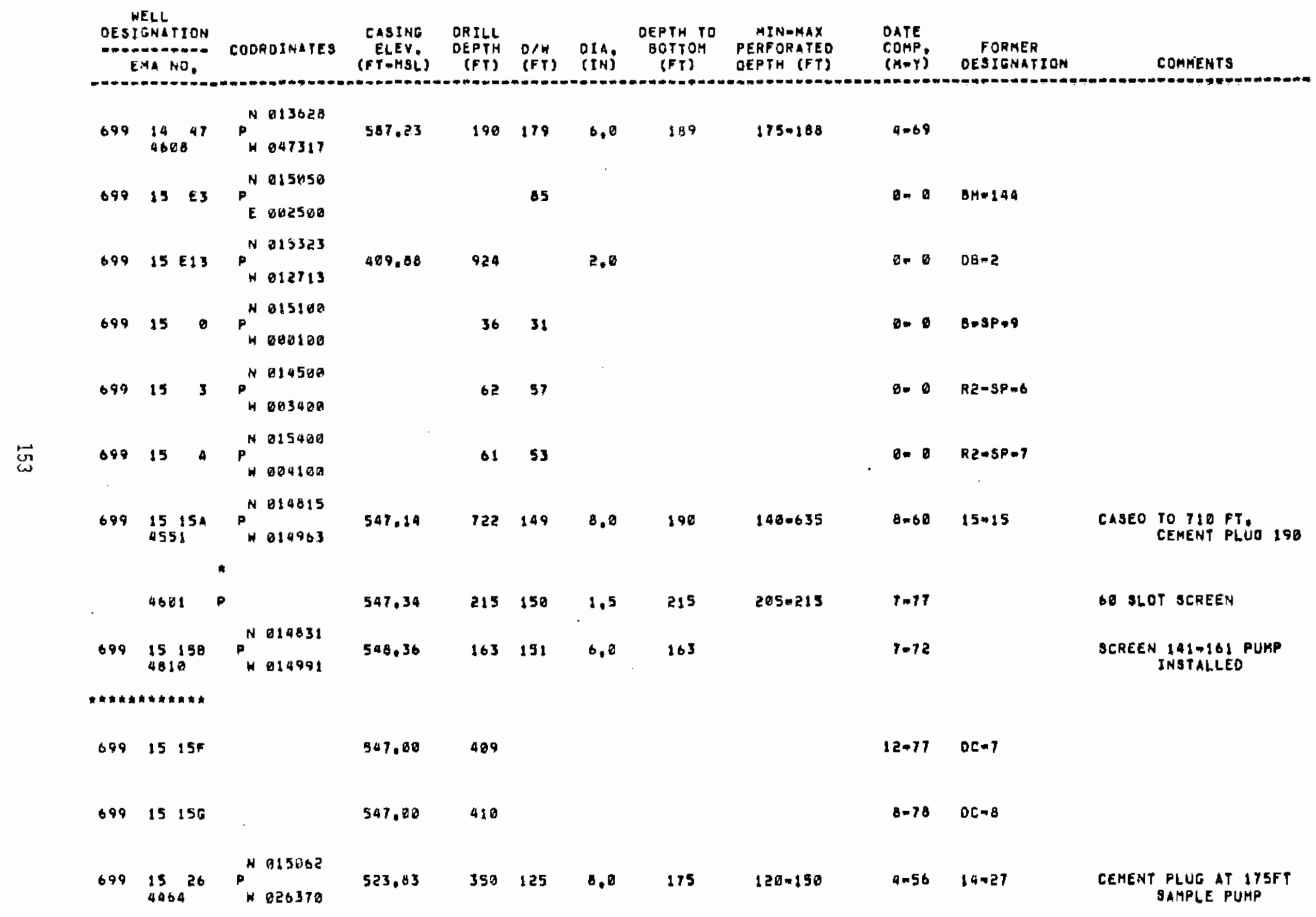




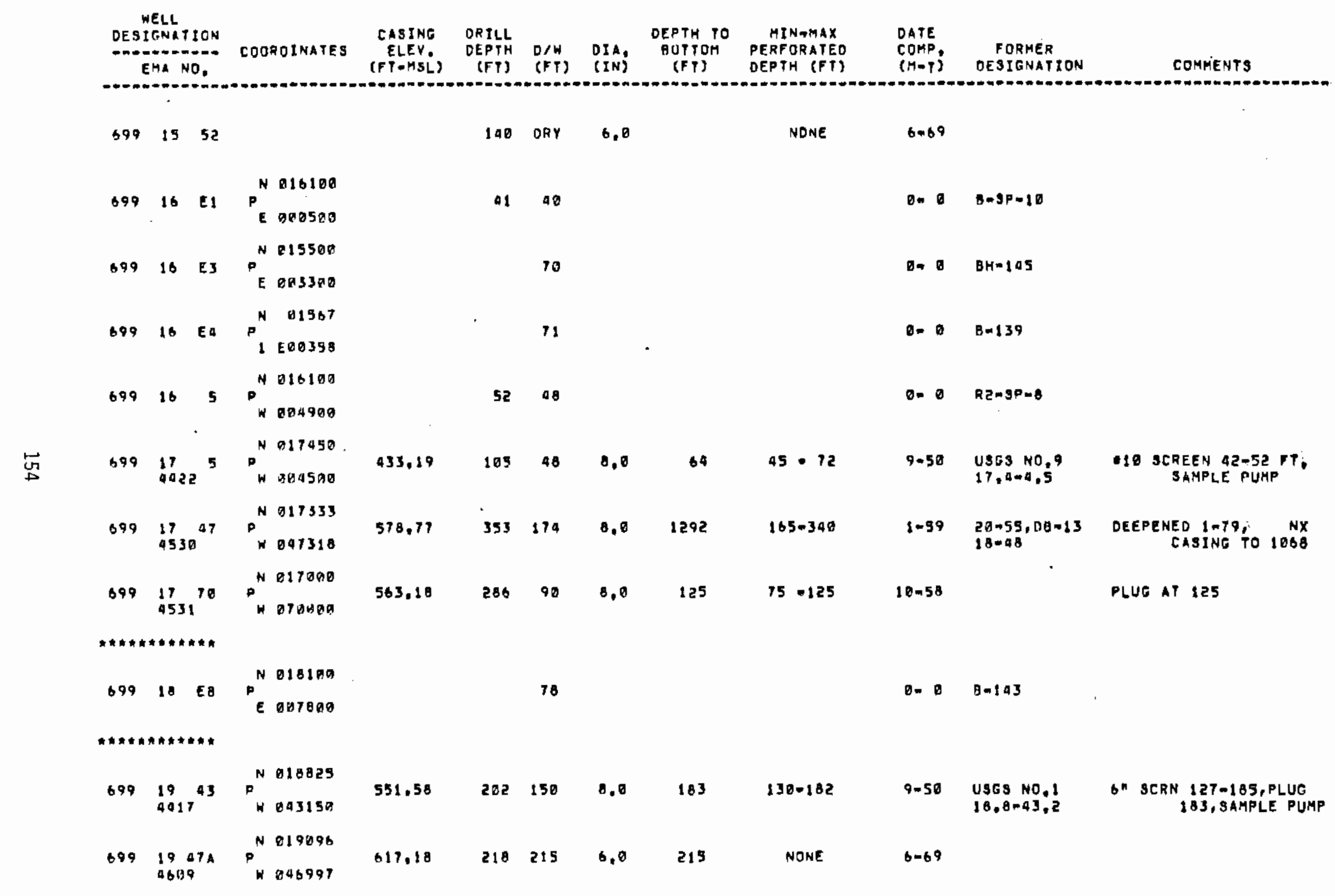




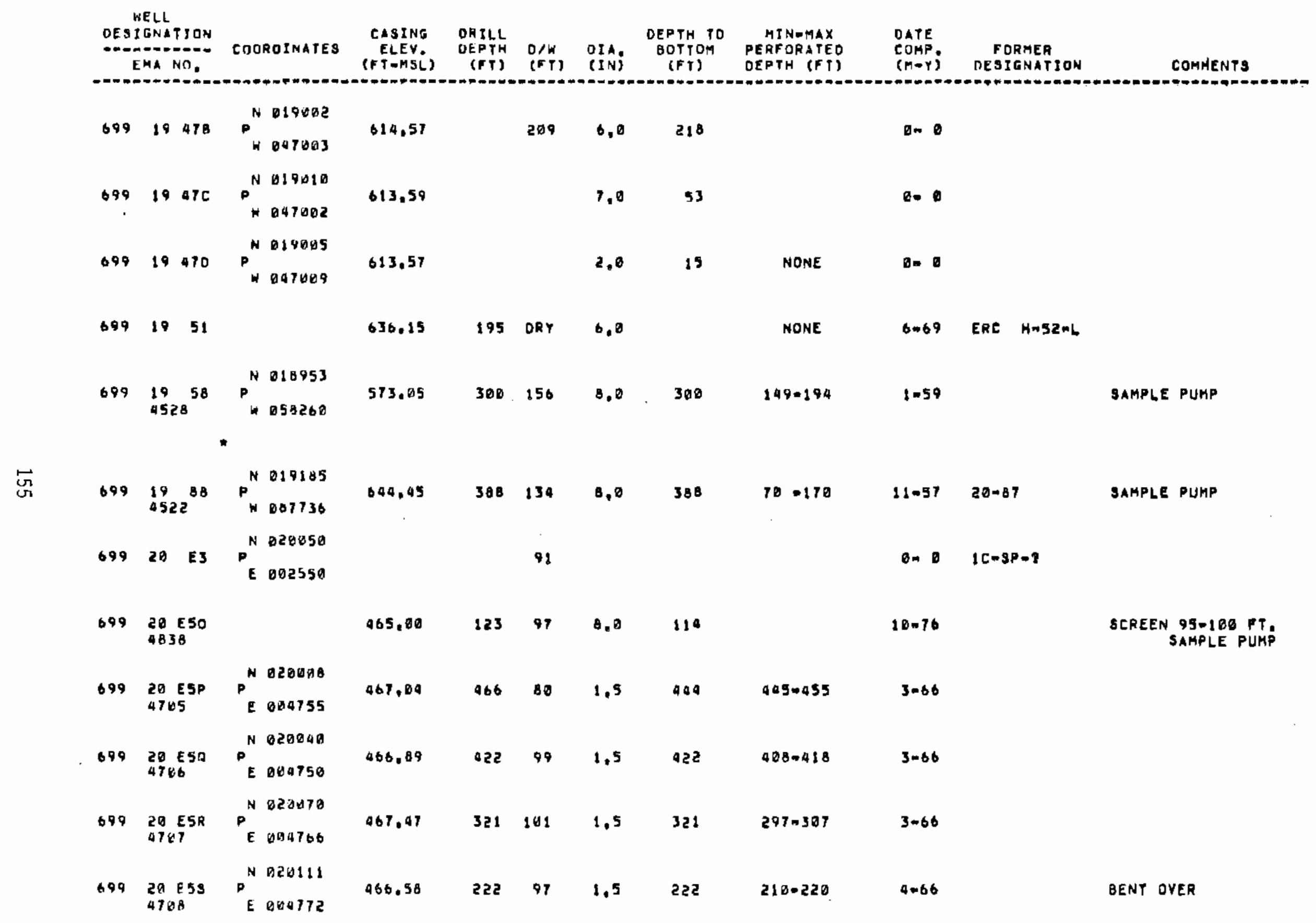




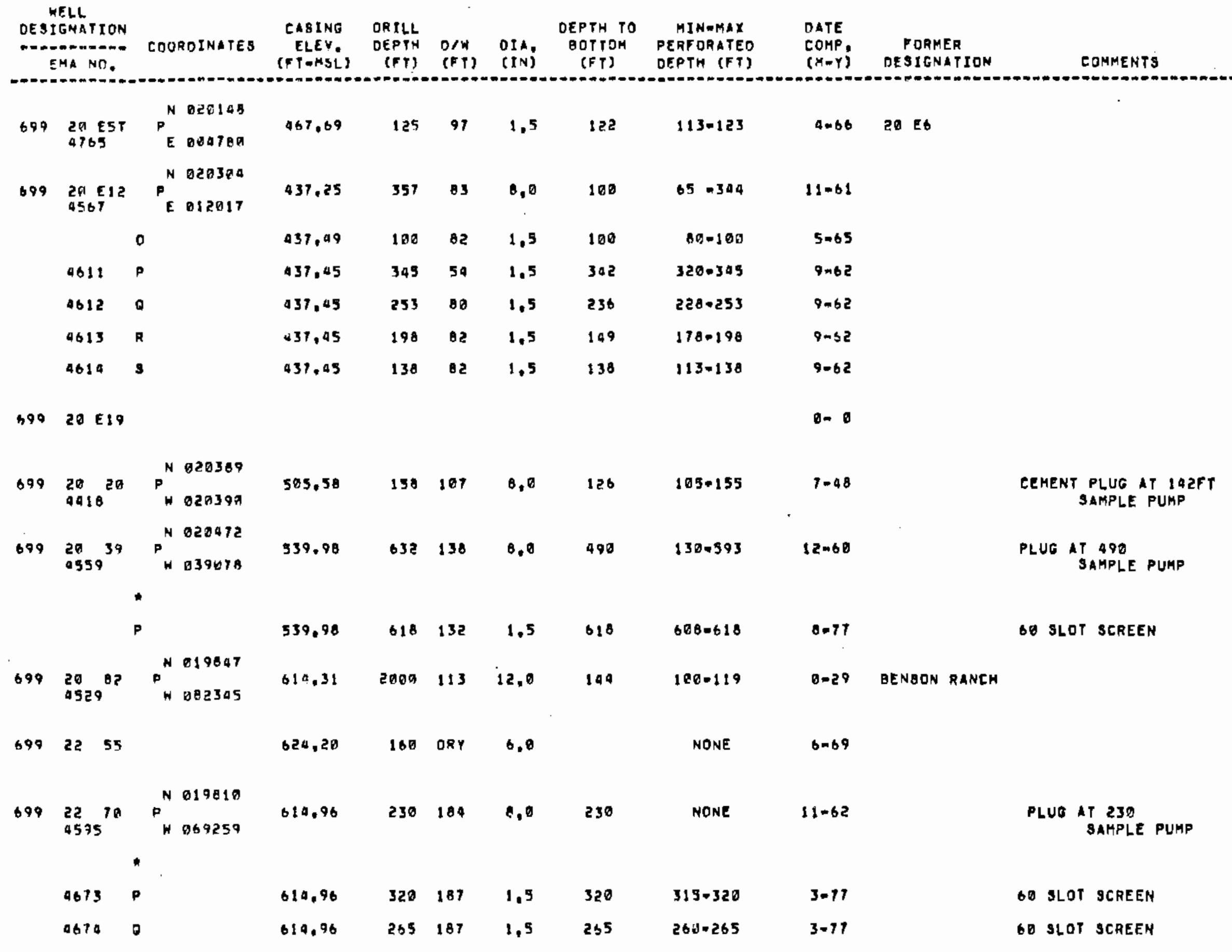




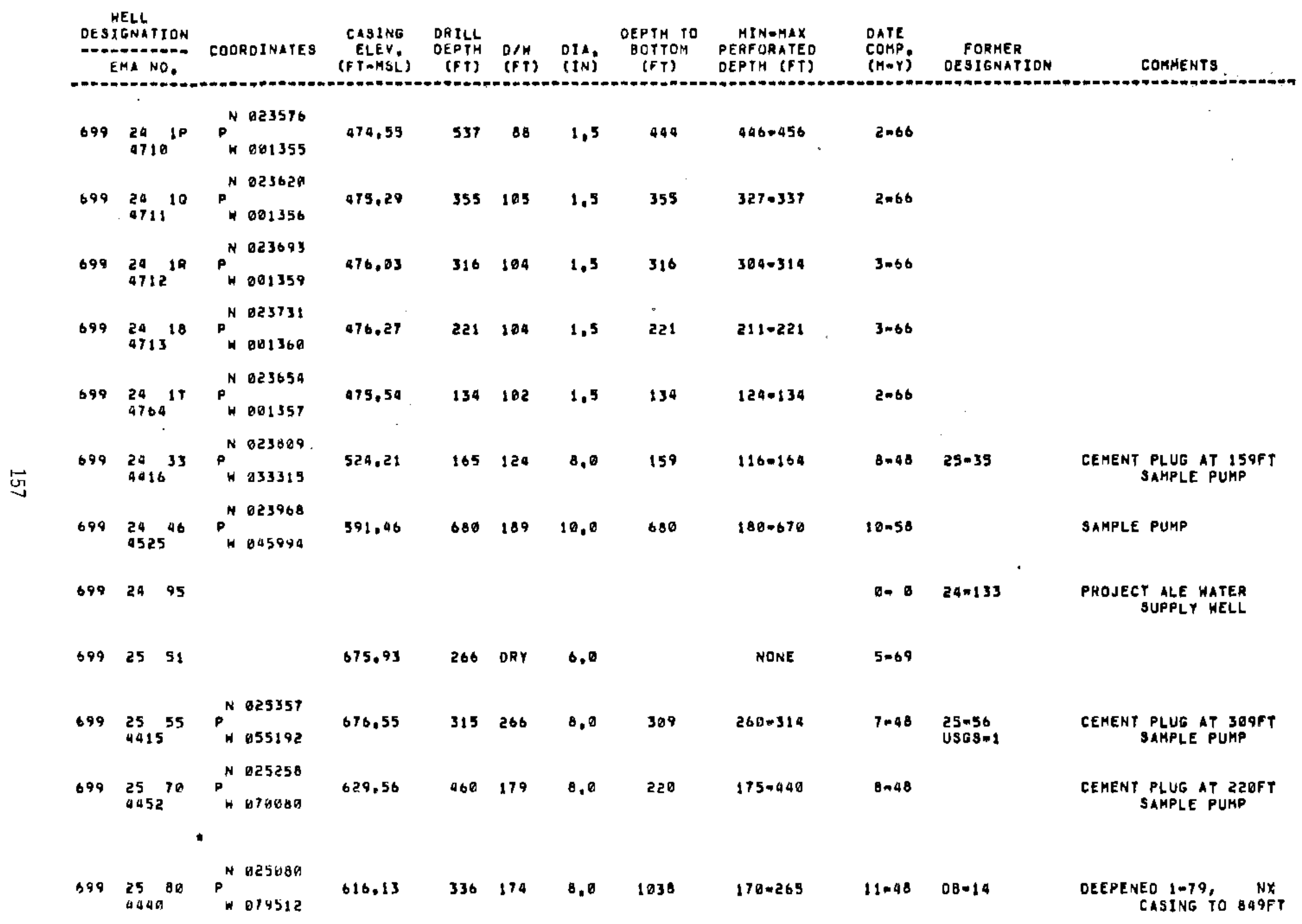




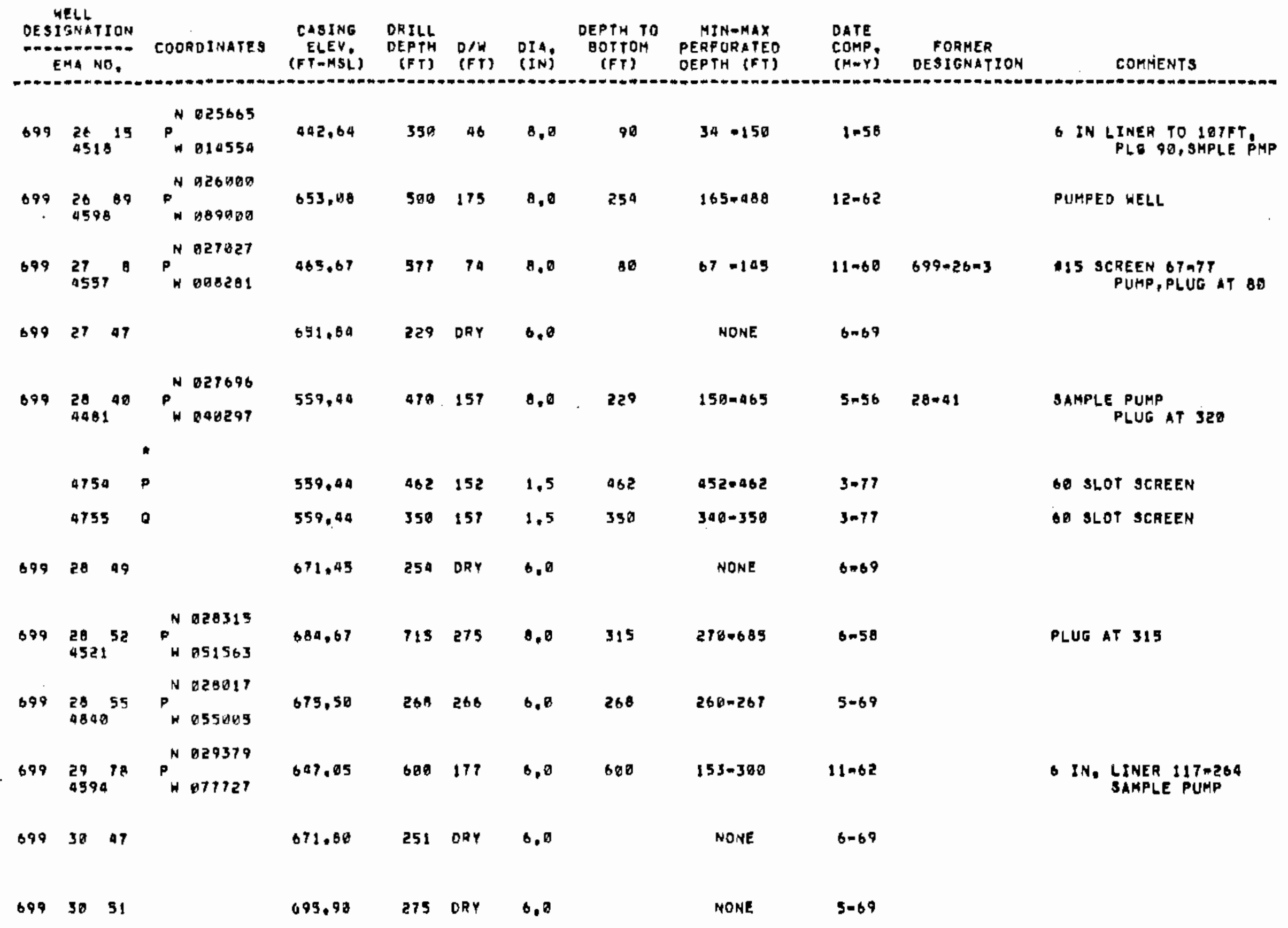




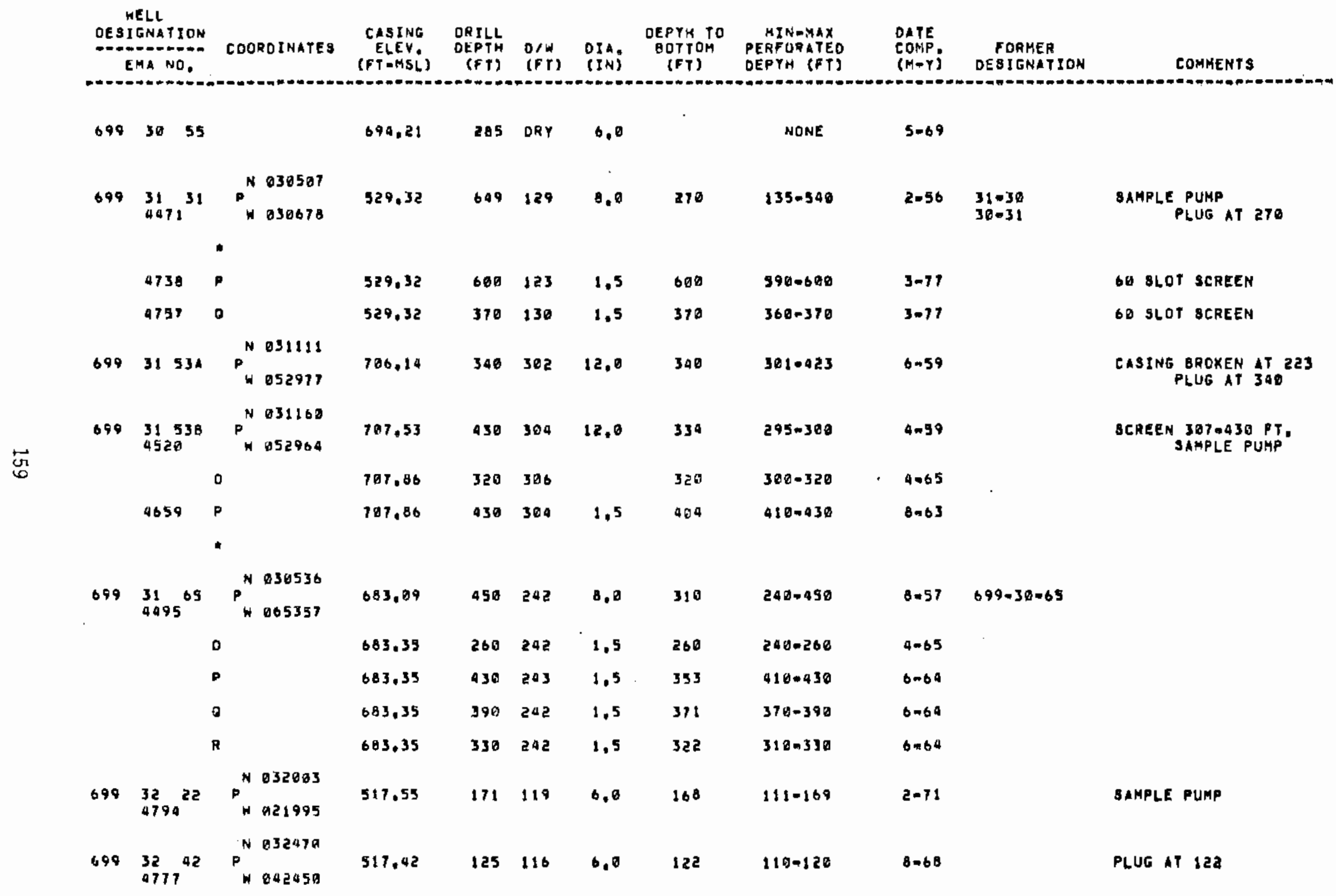




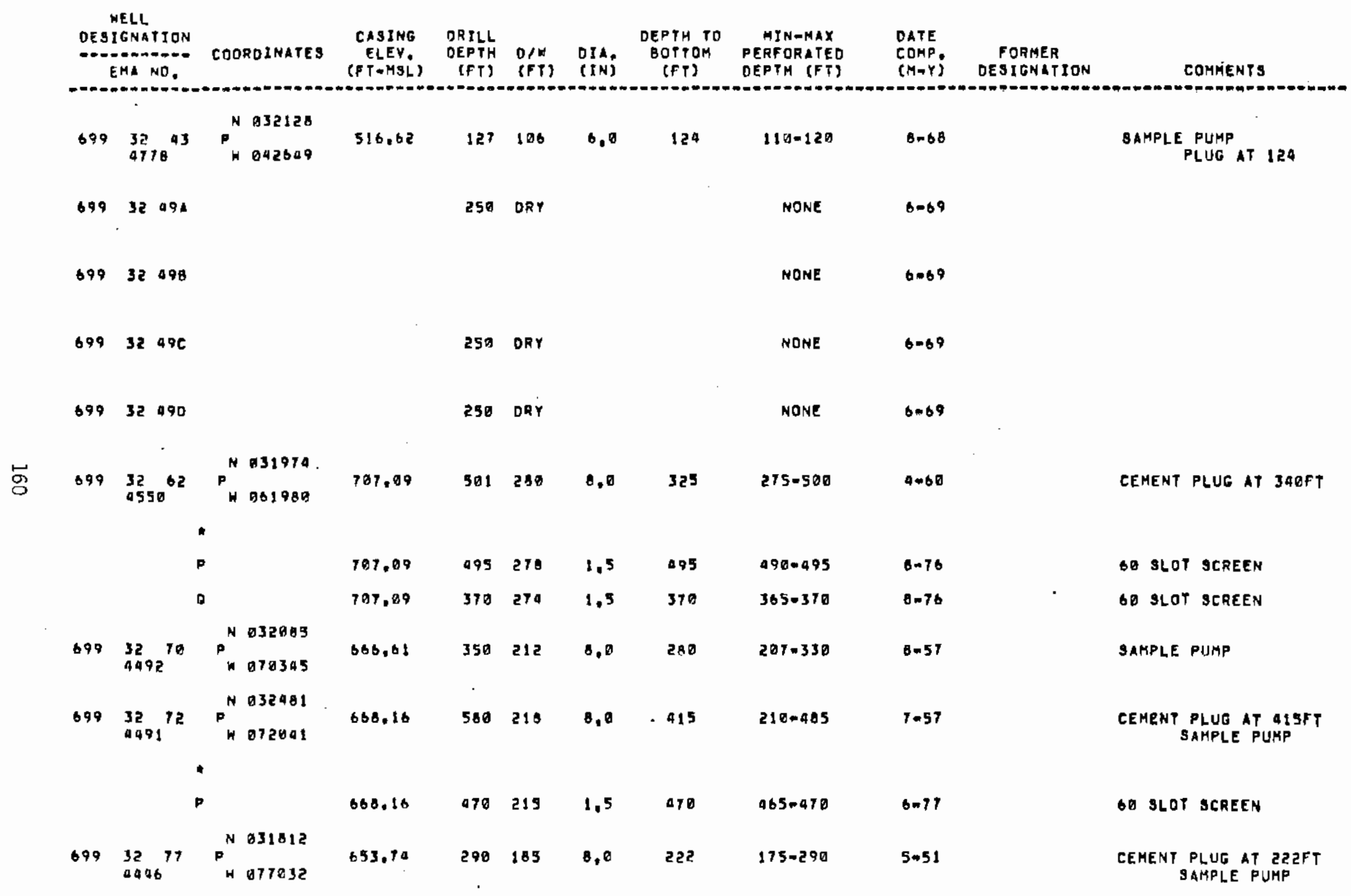




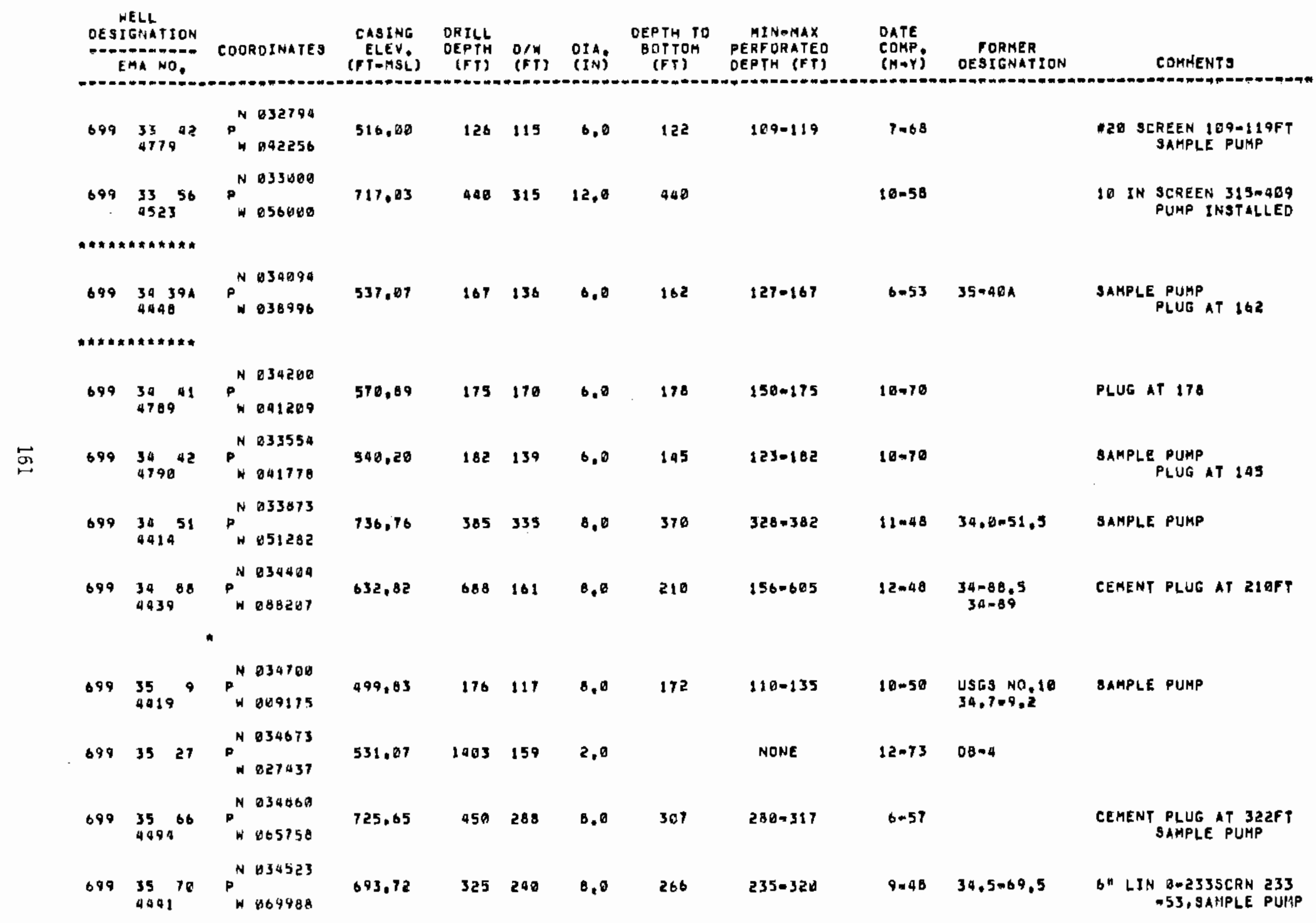




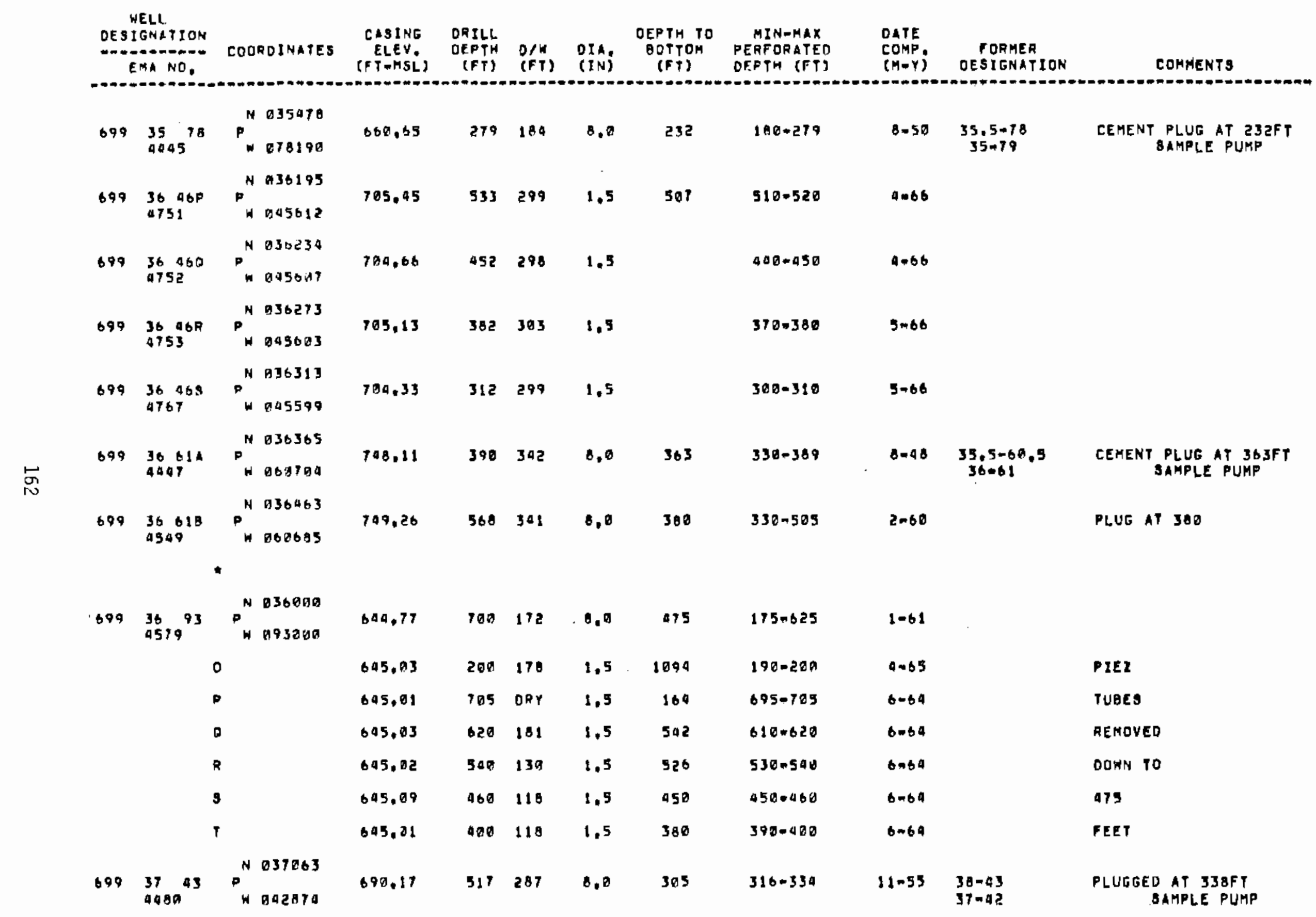




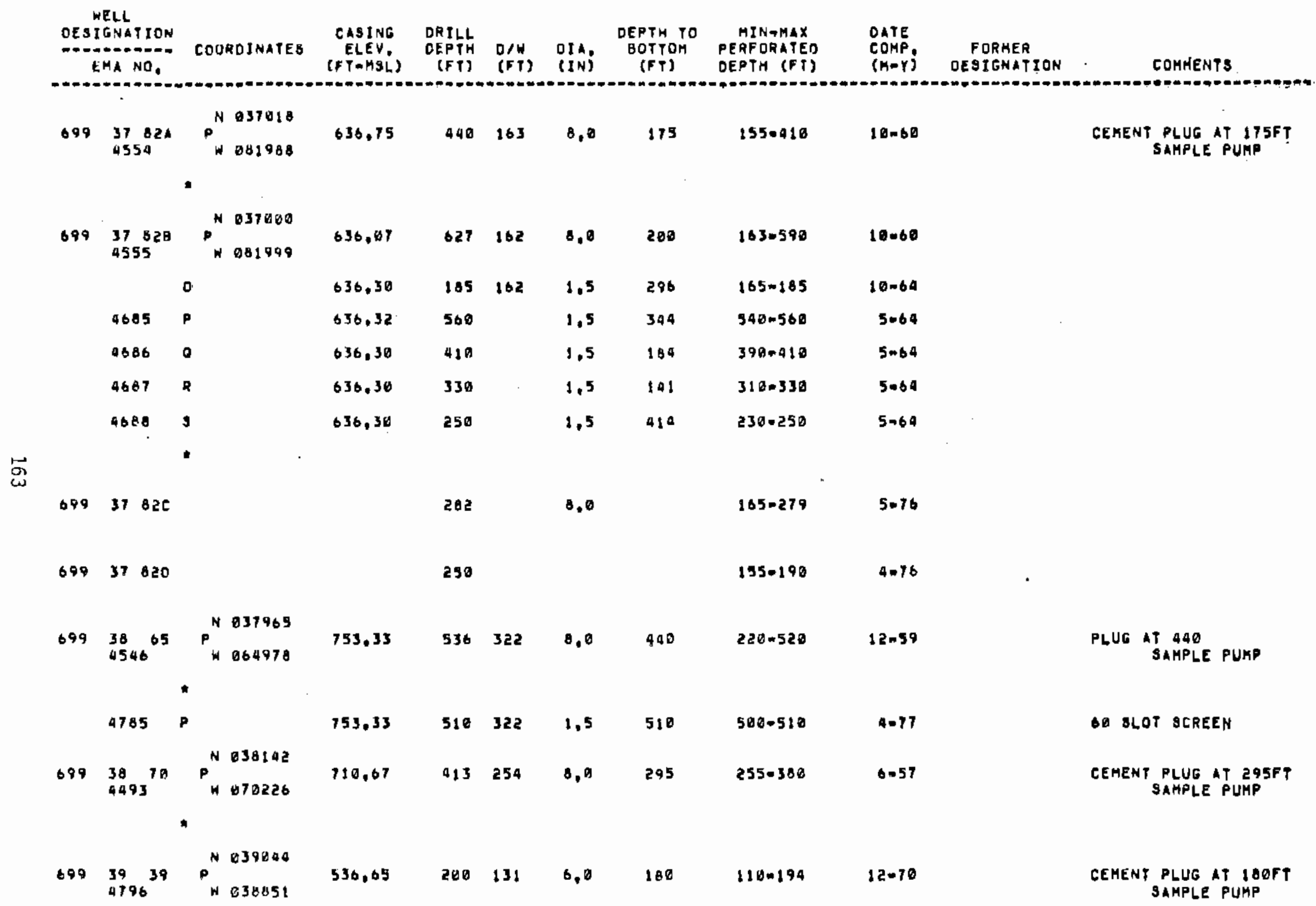




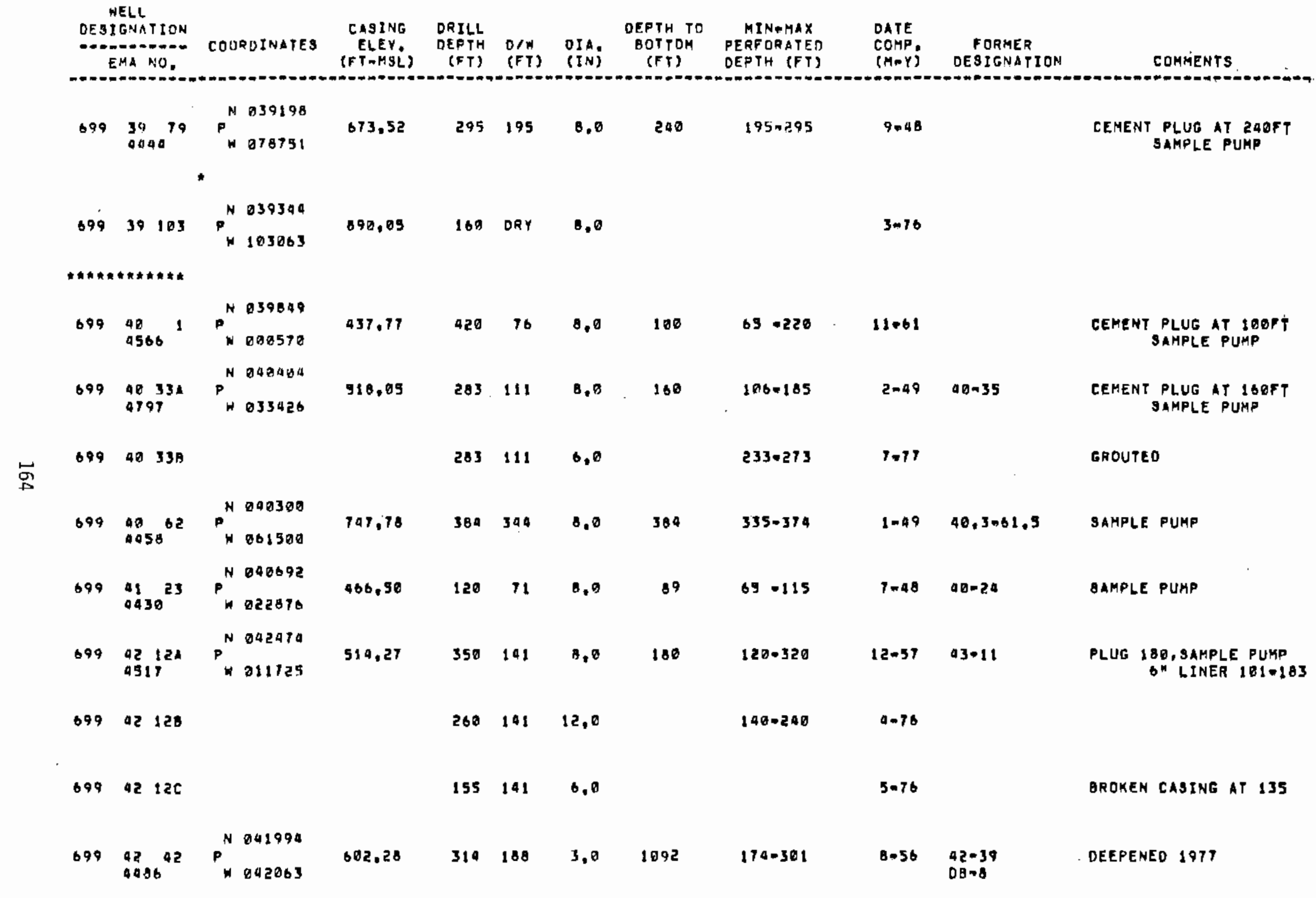




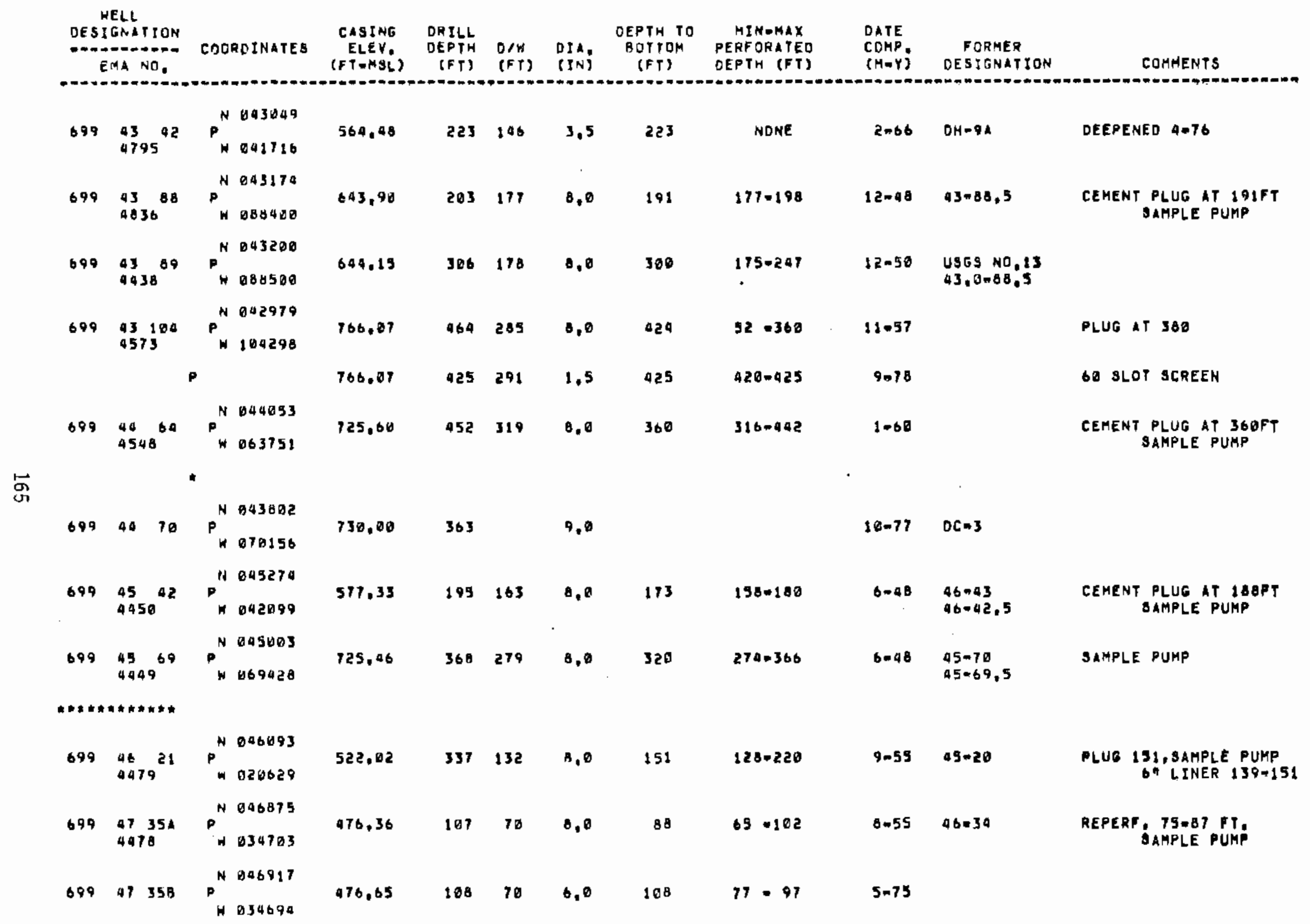




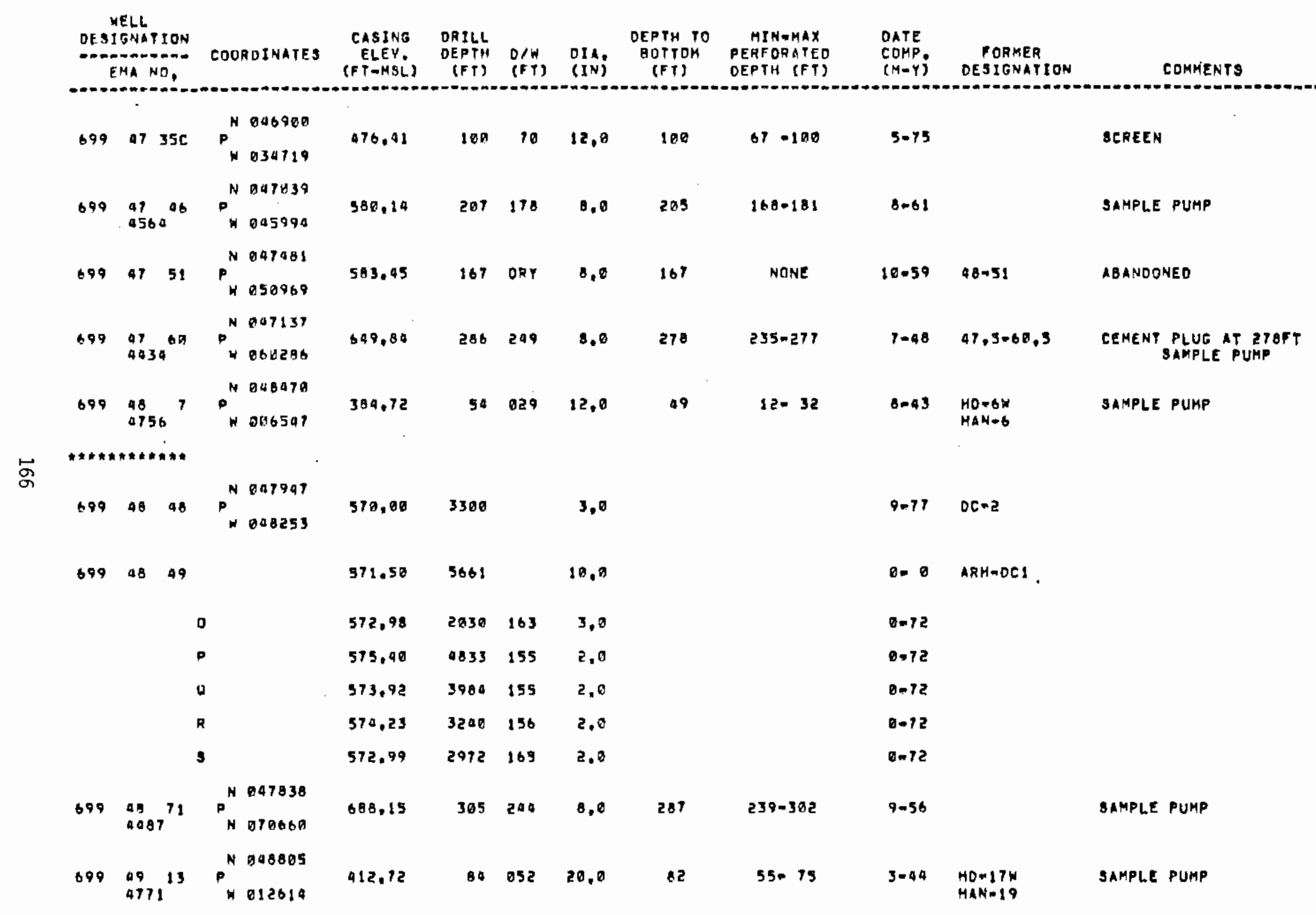




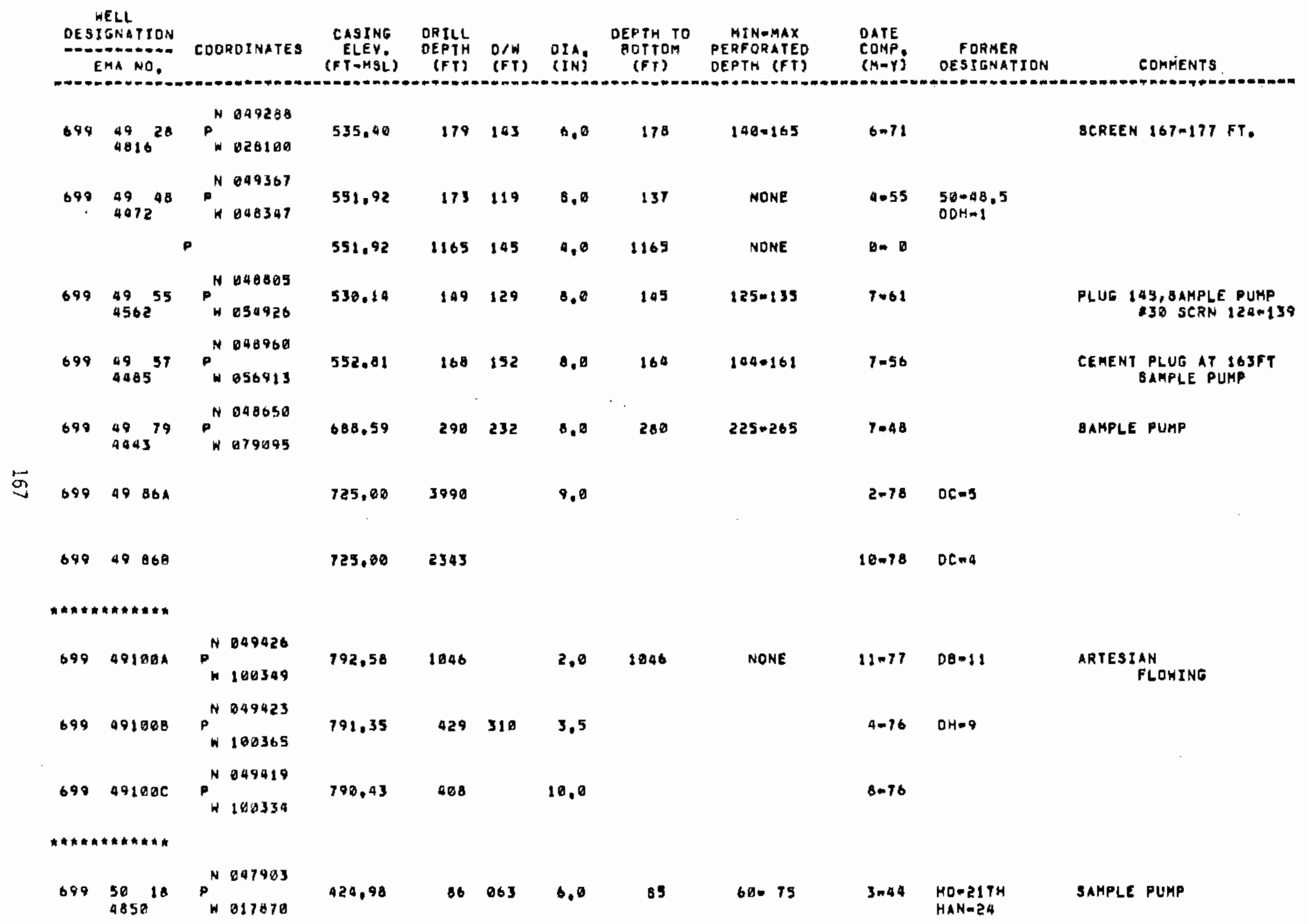




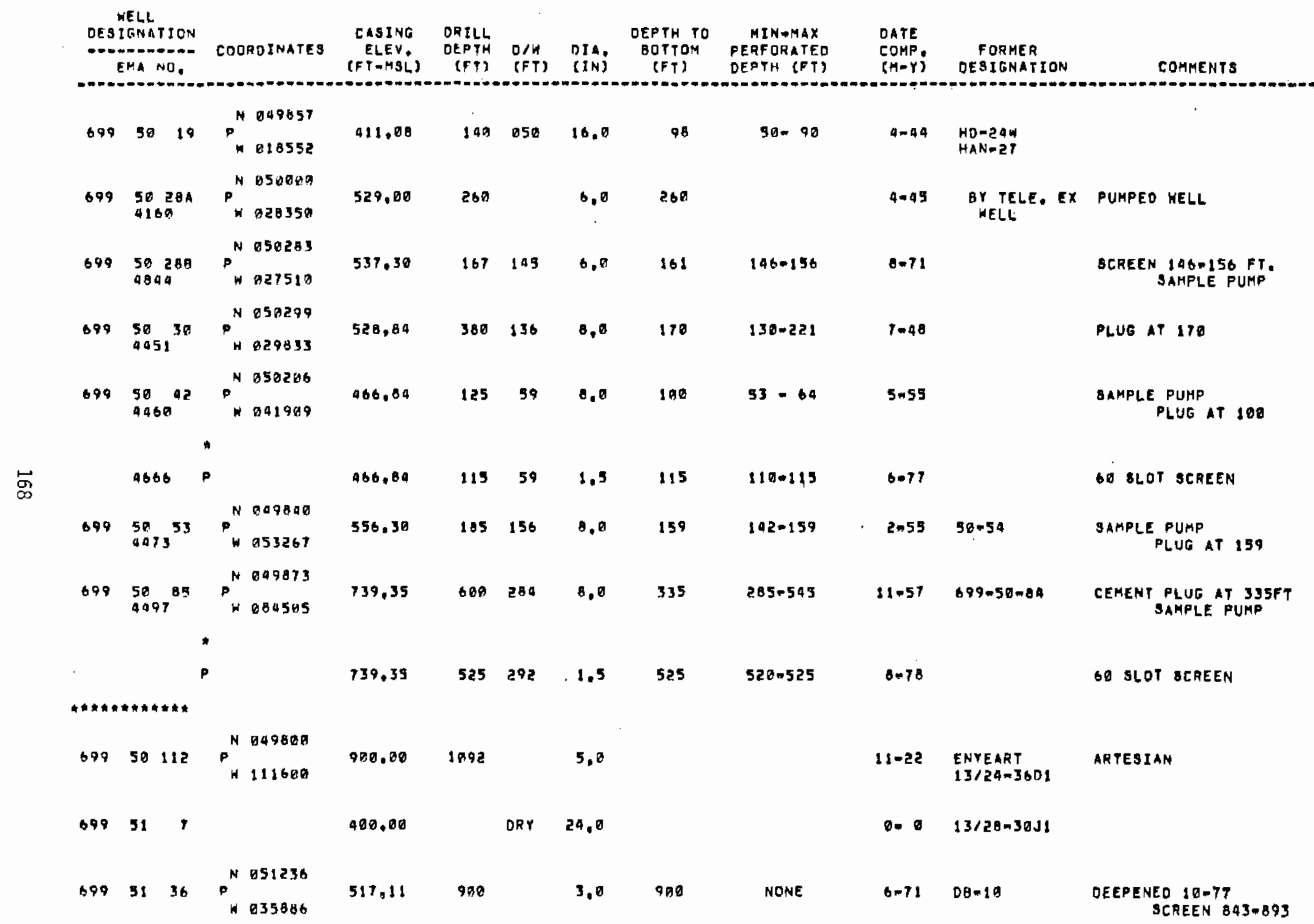




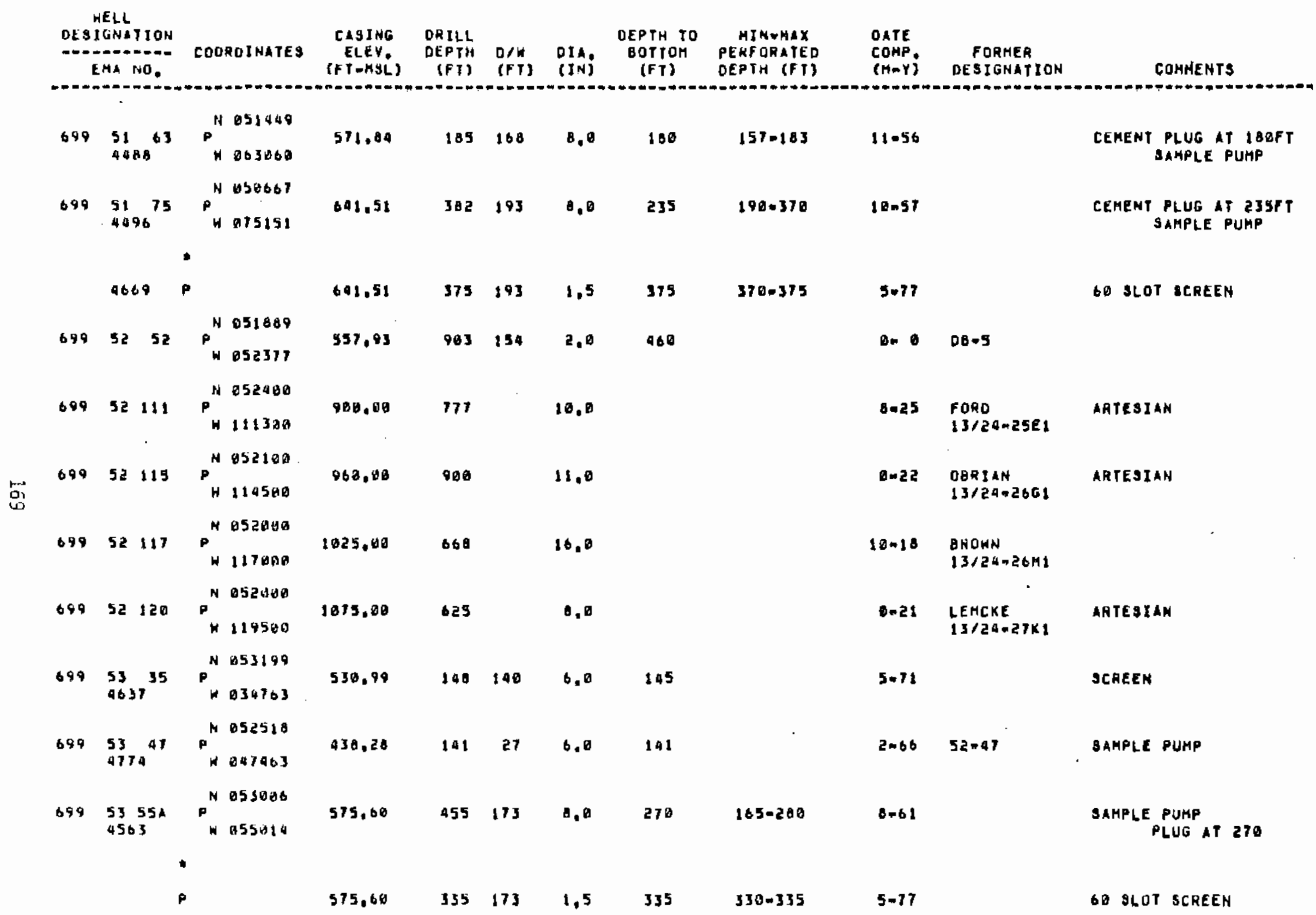




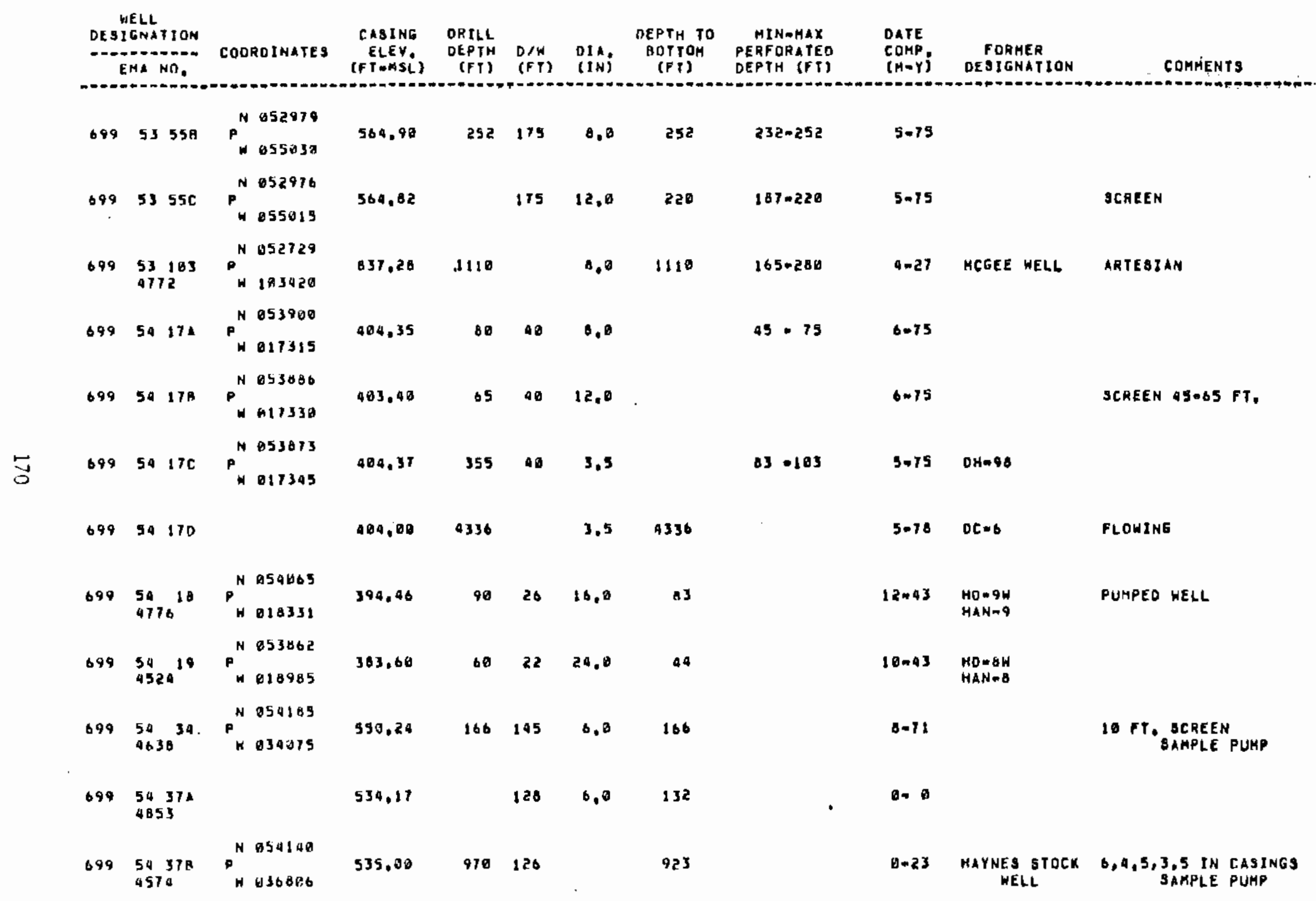




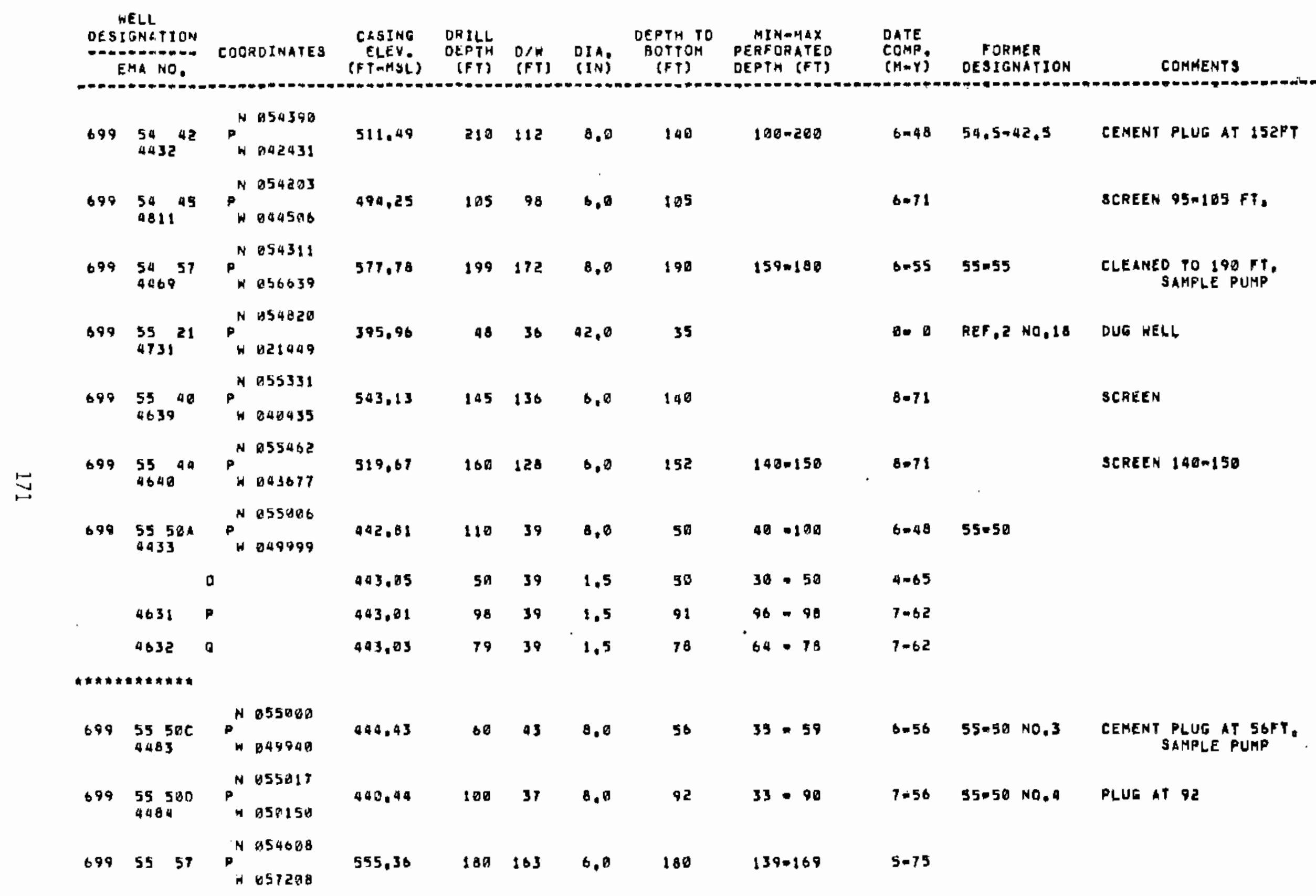




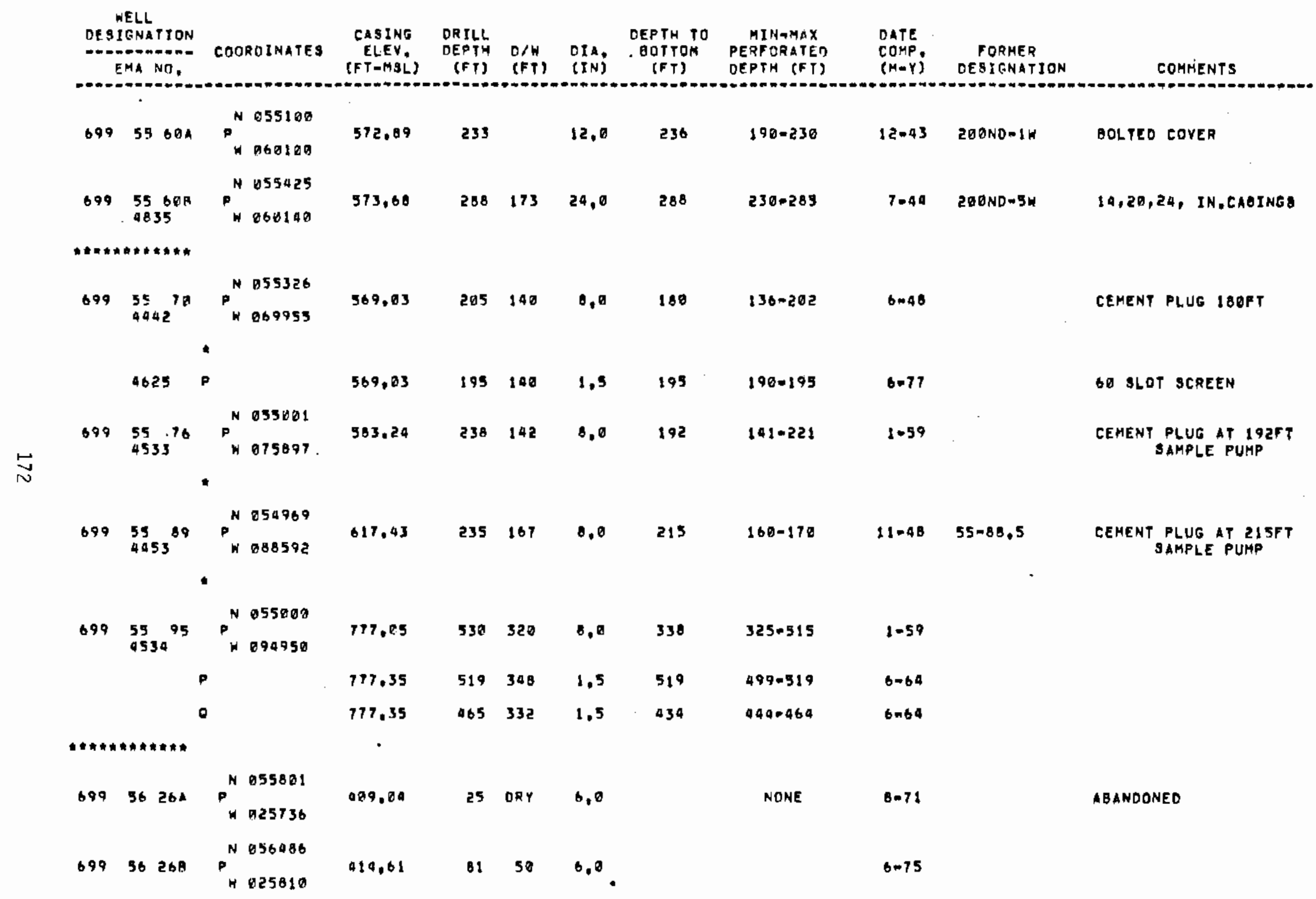




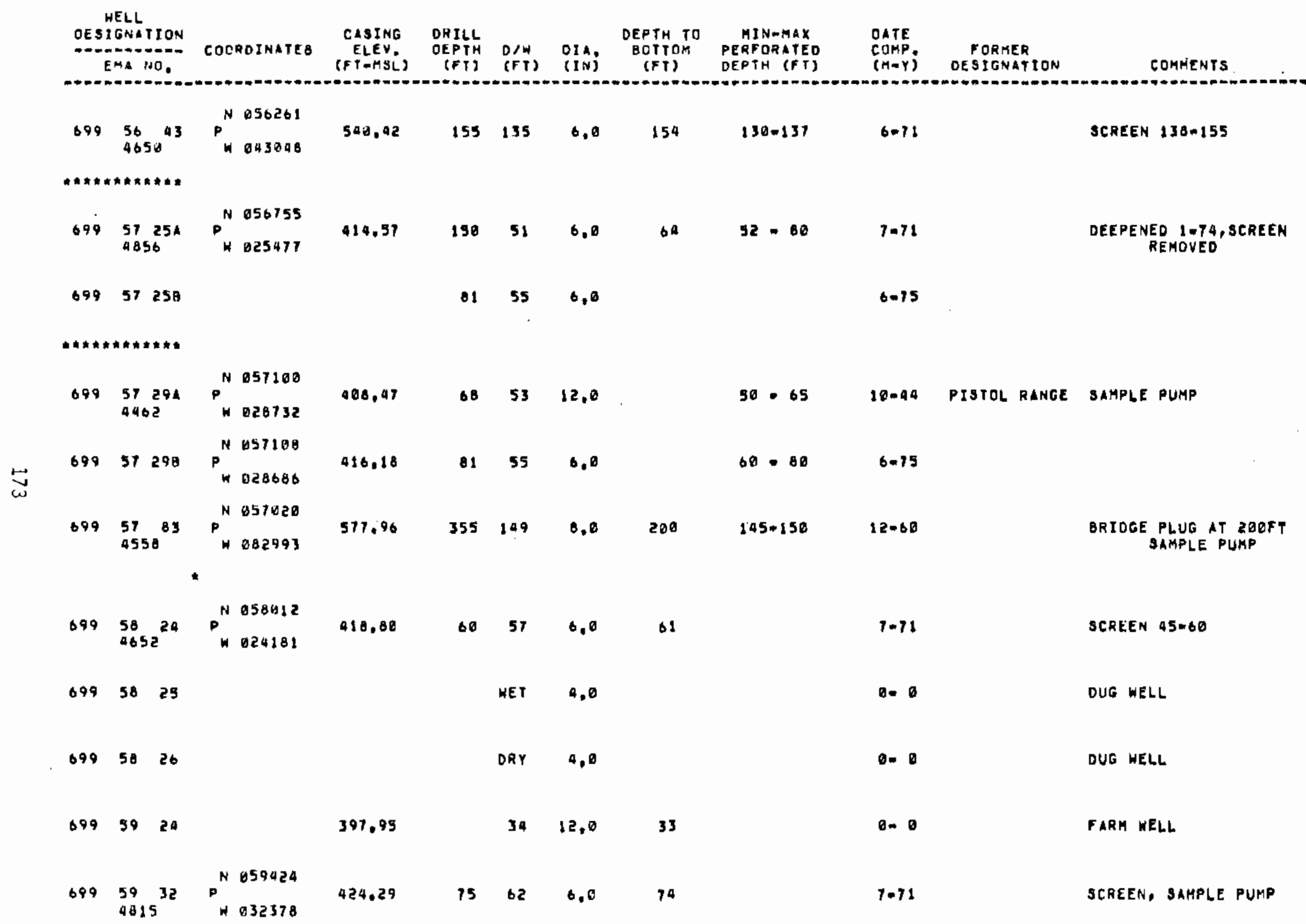




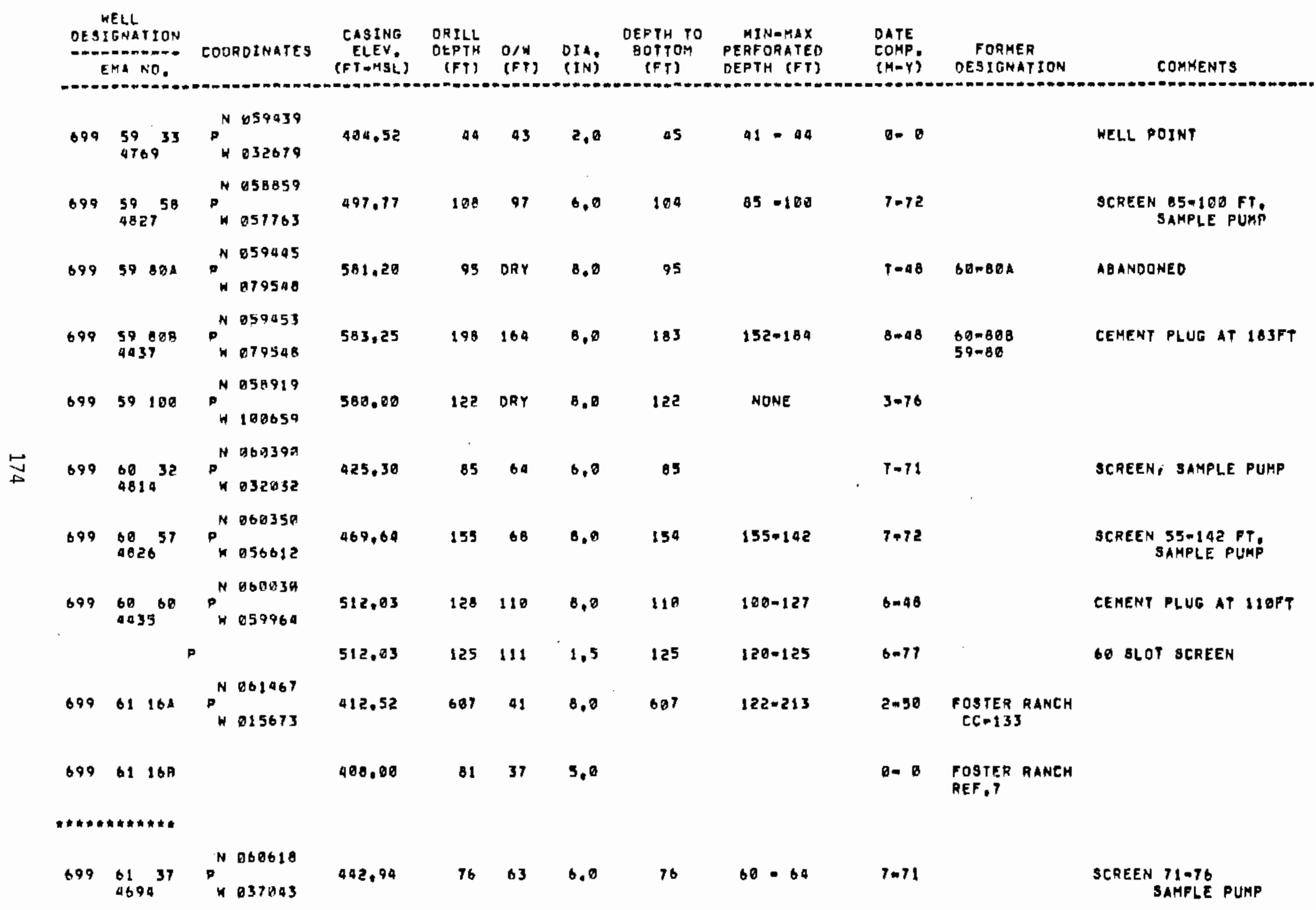




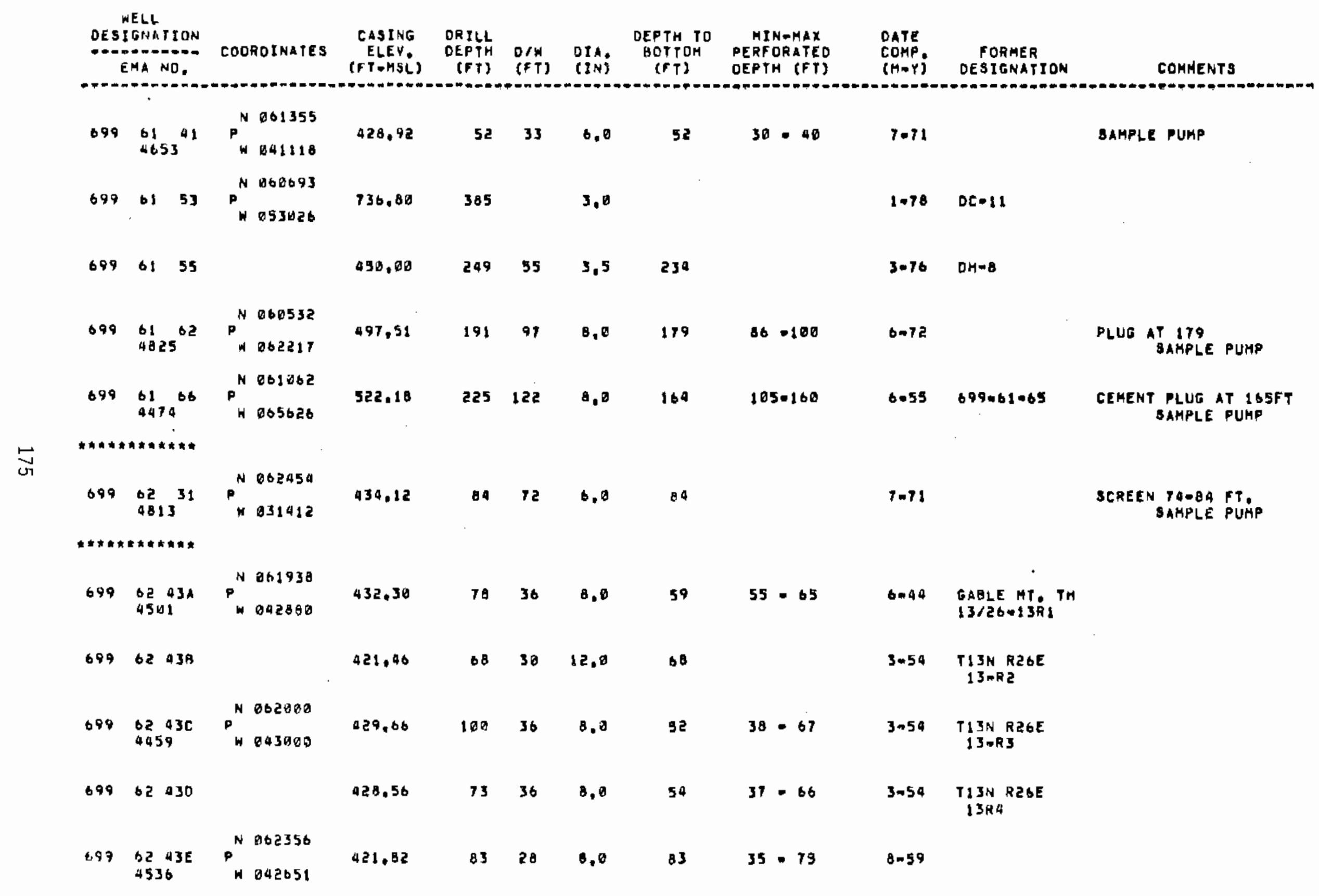




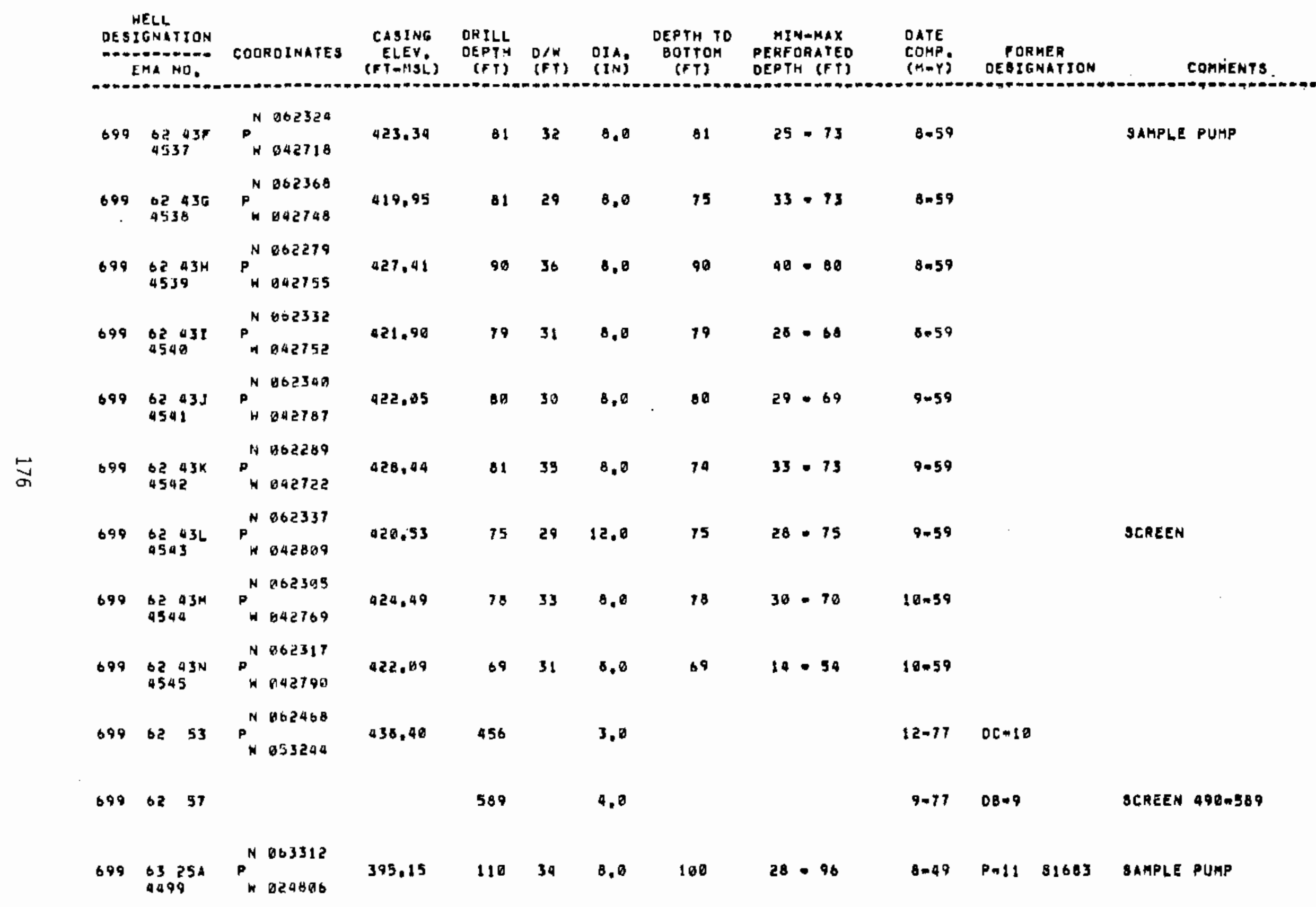




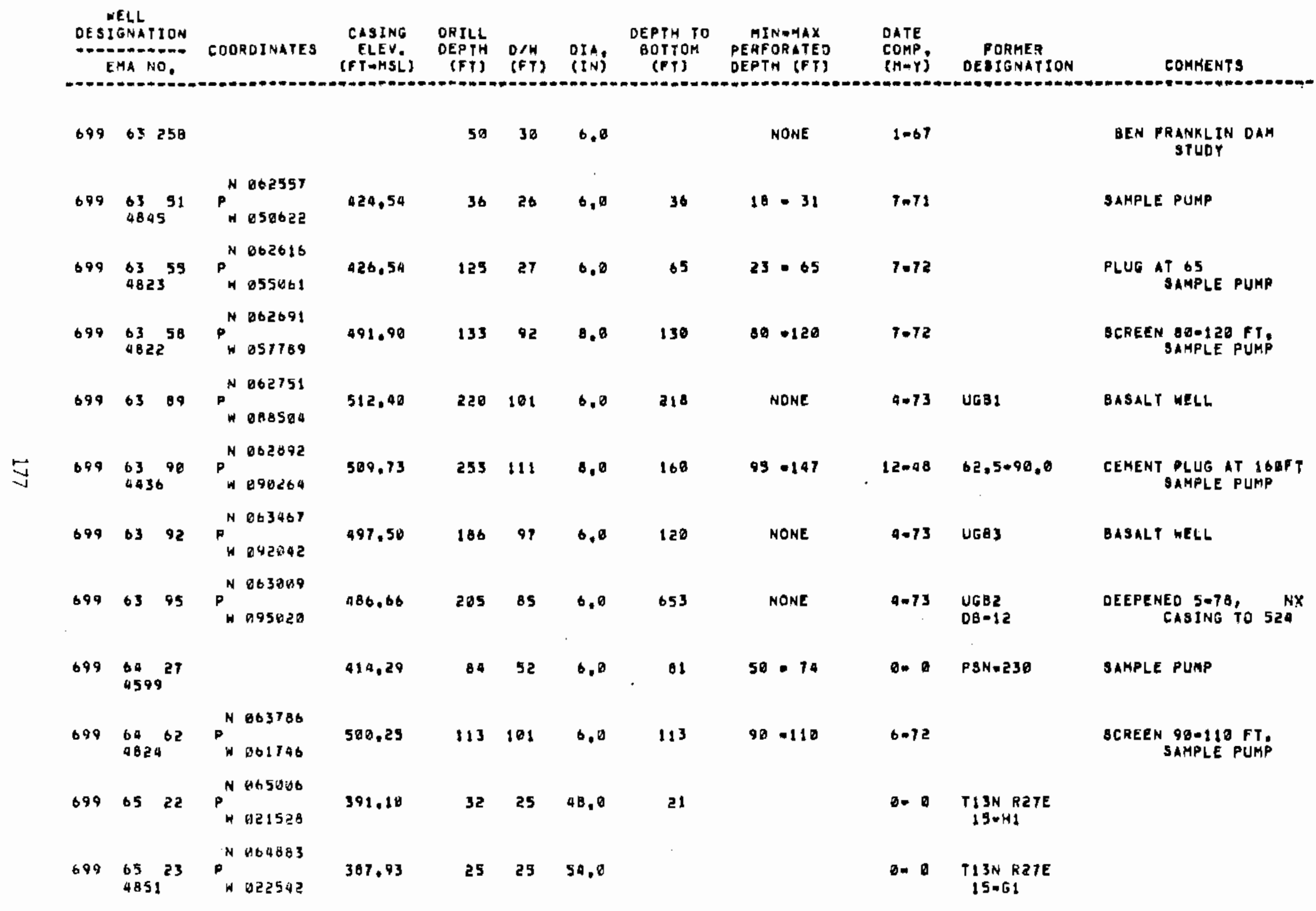


WELL

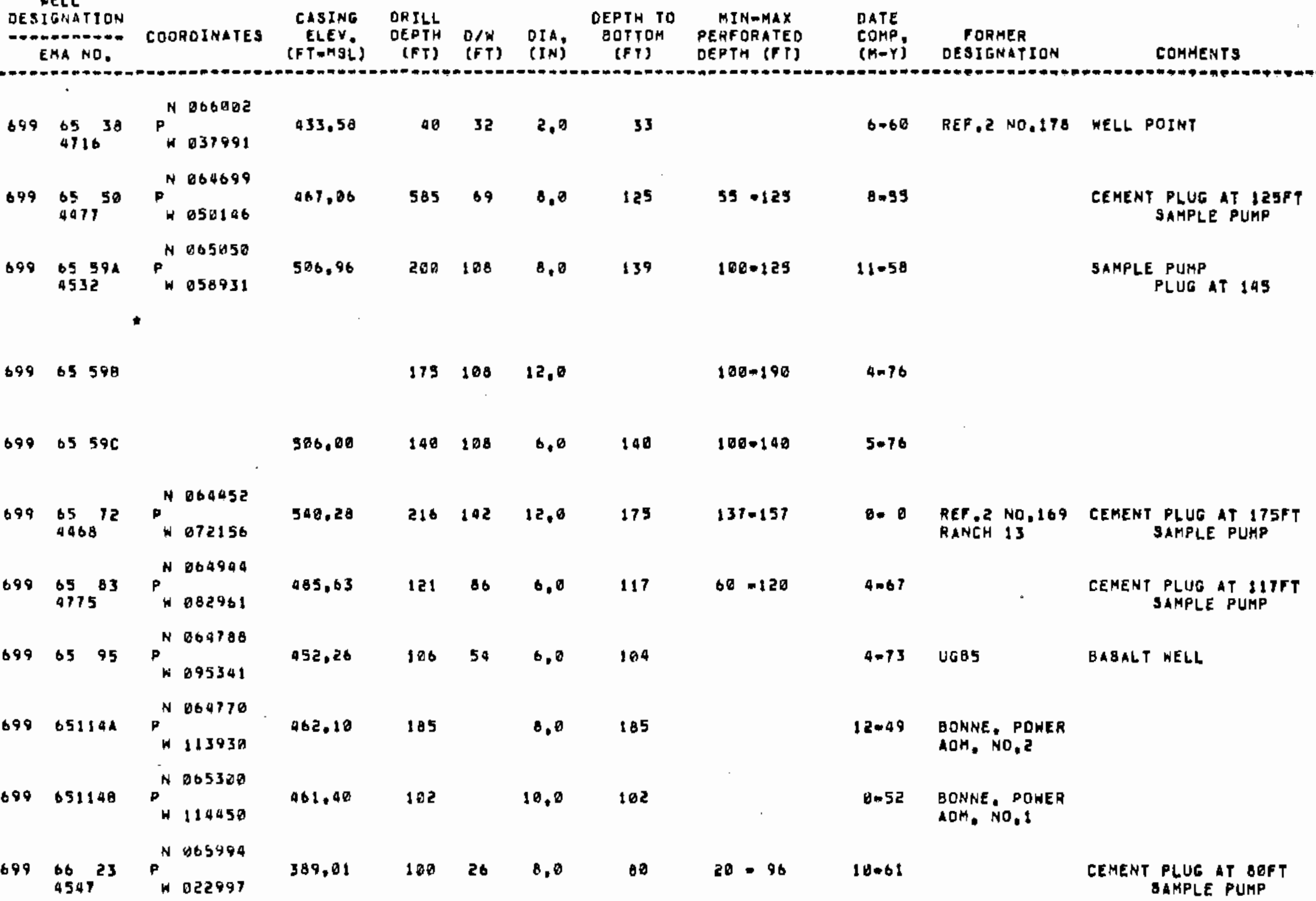




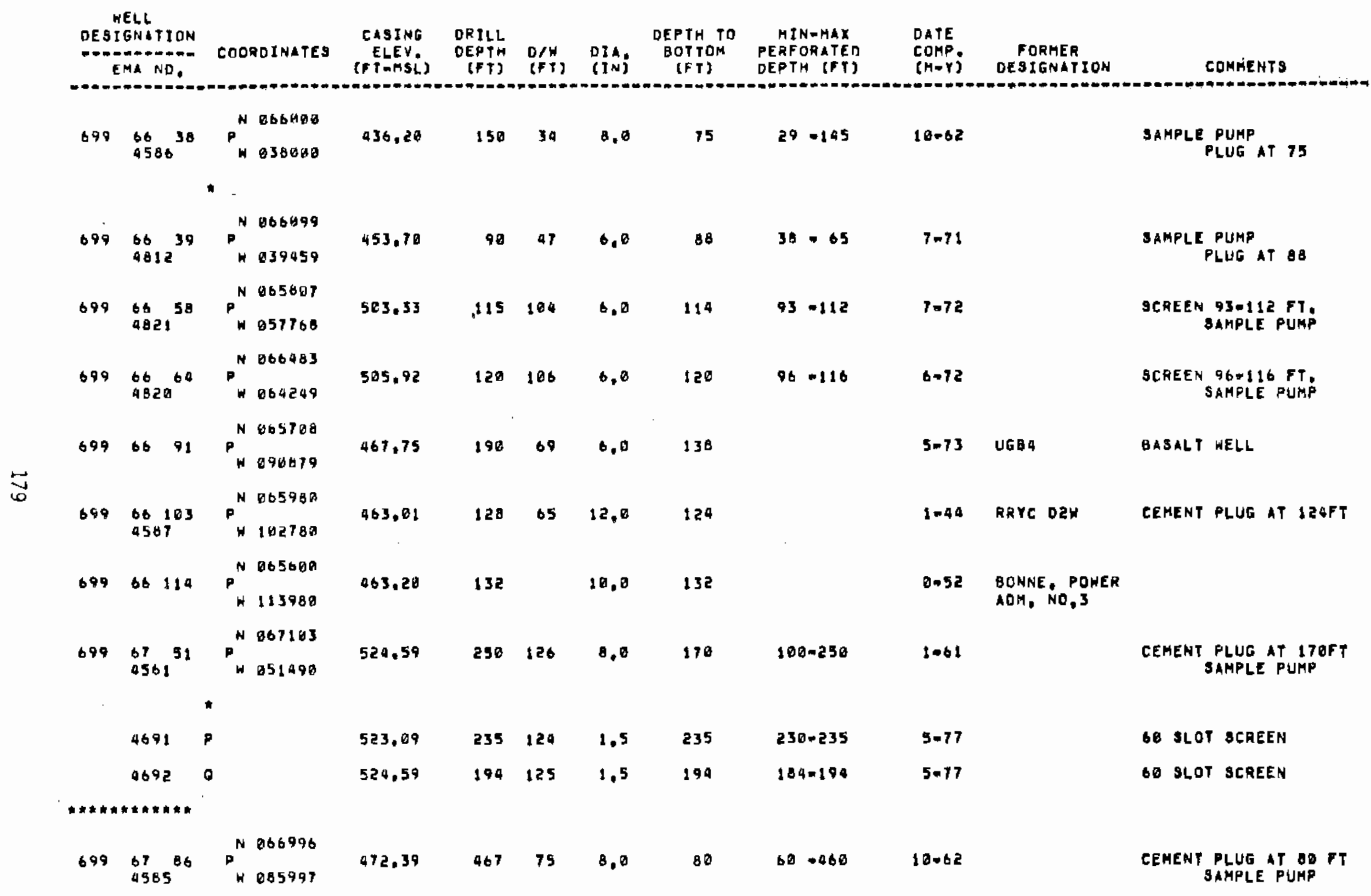




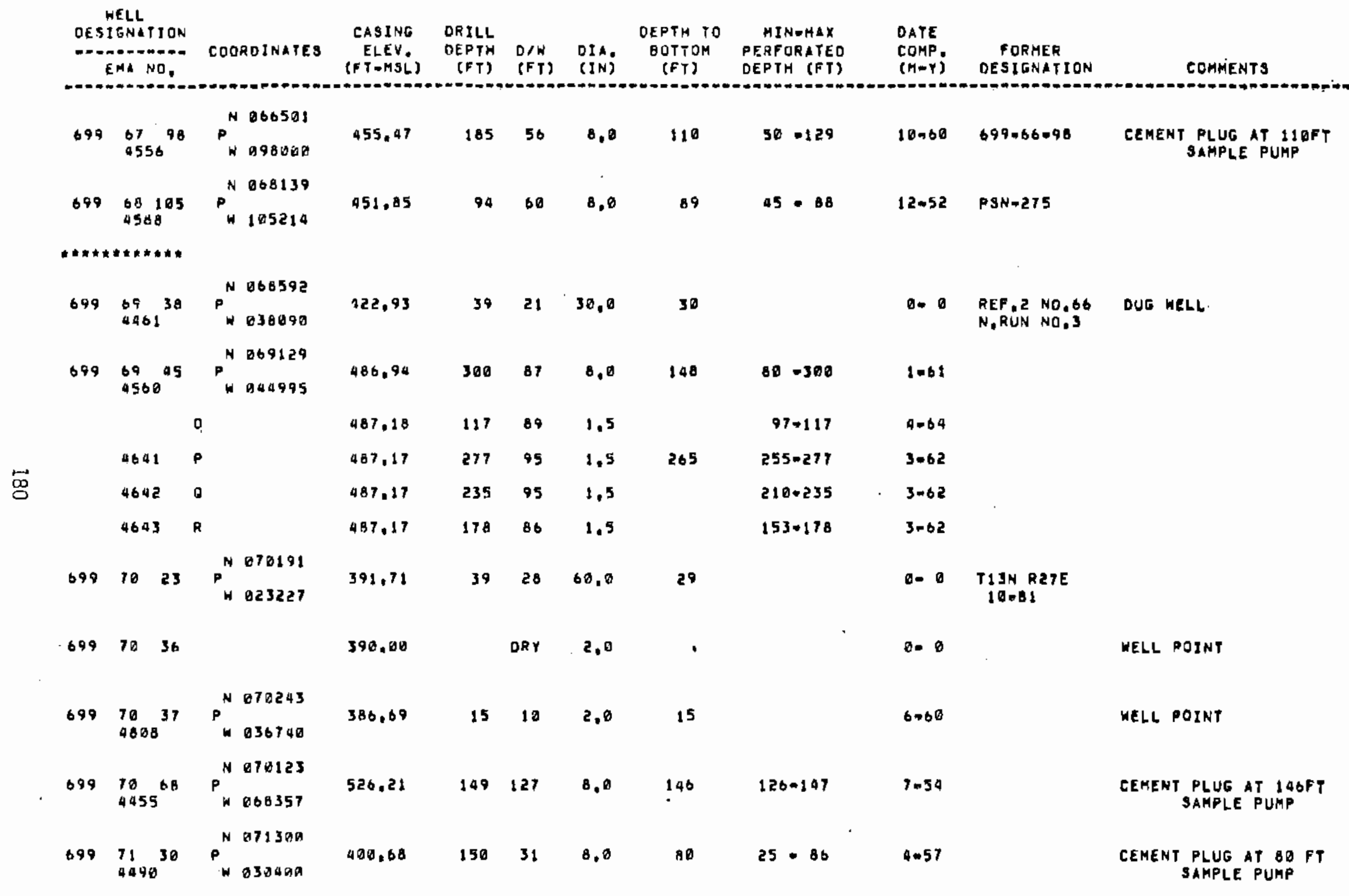




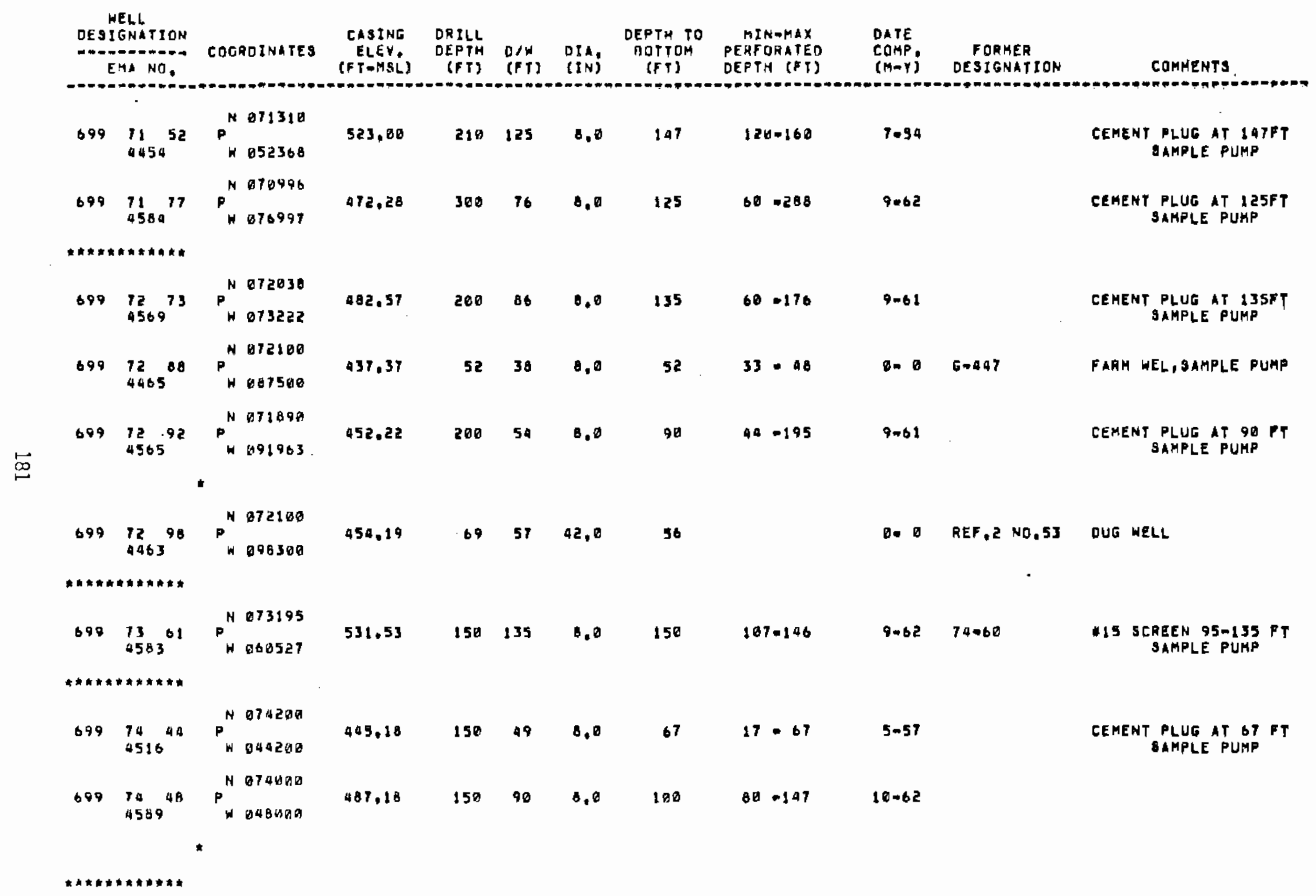




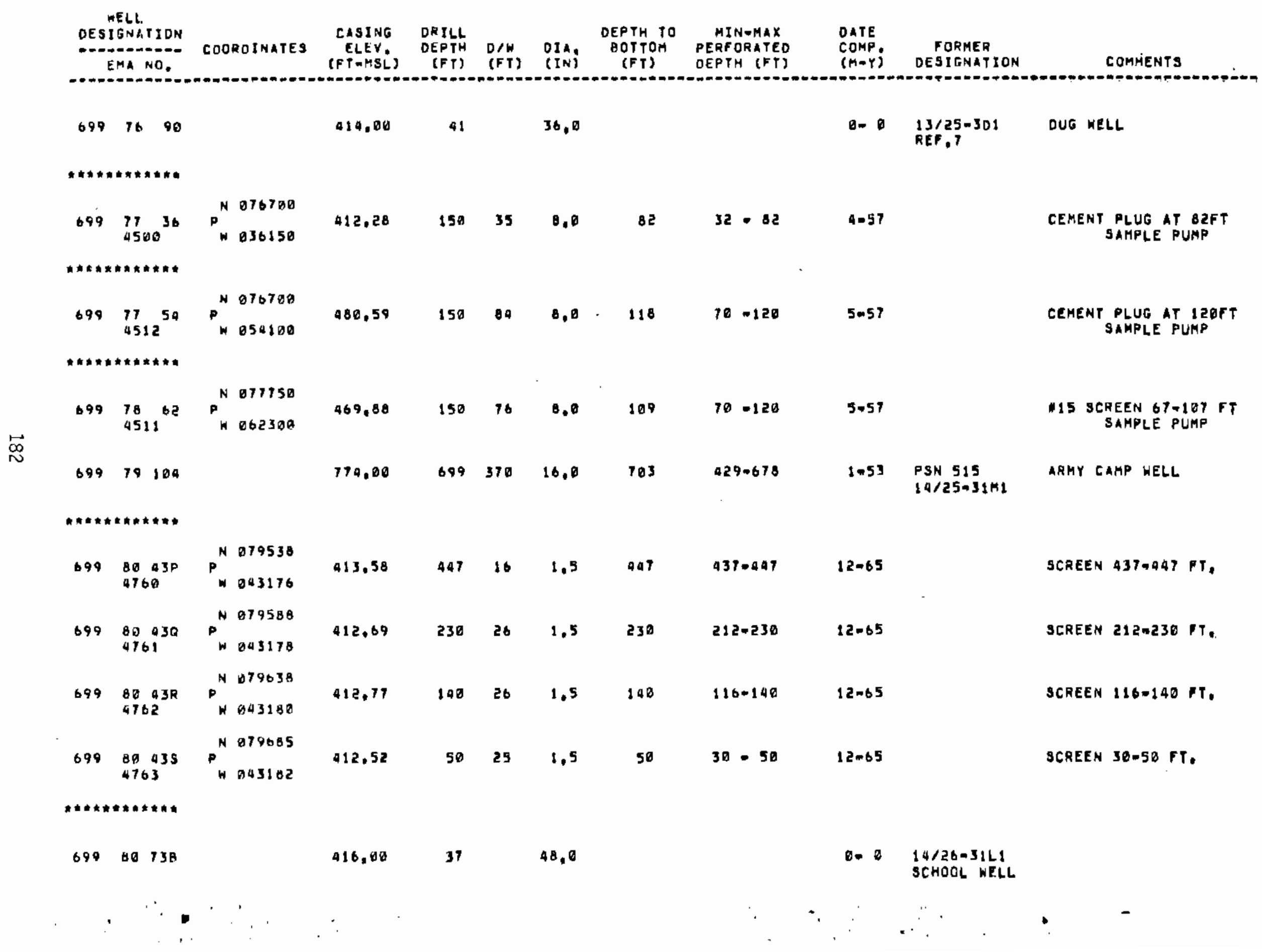




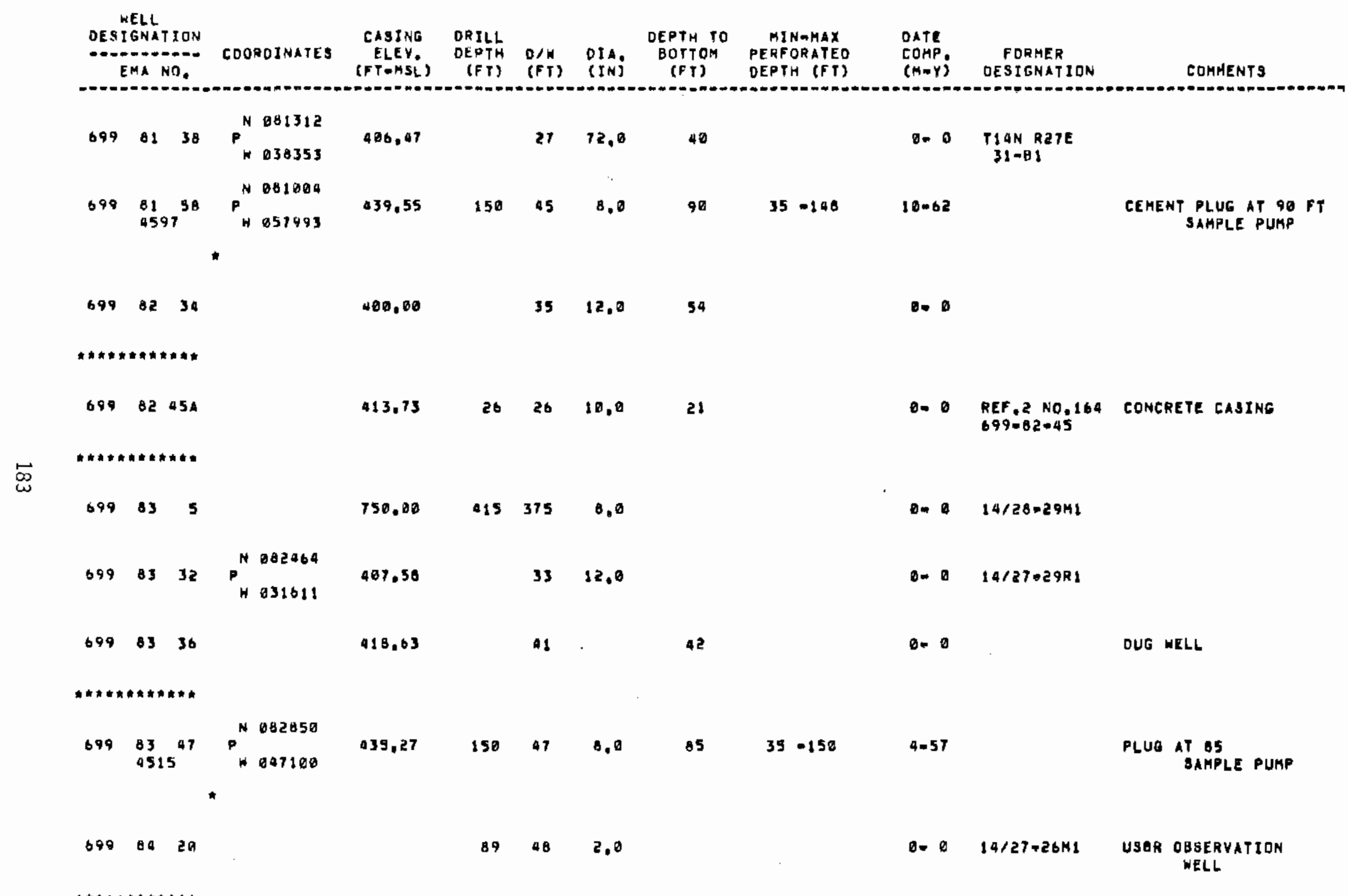




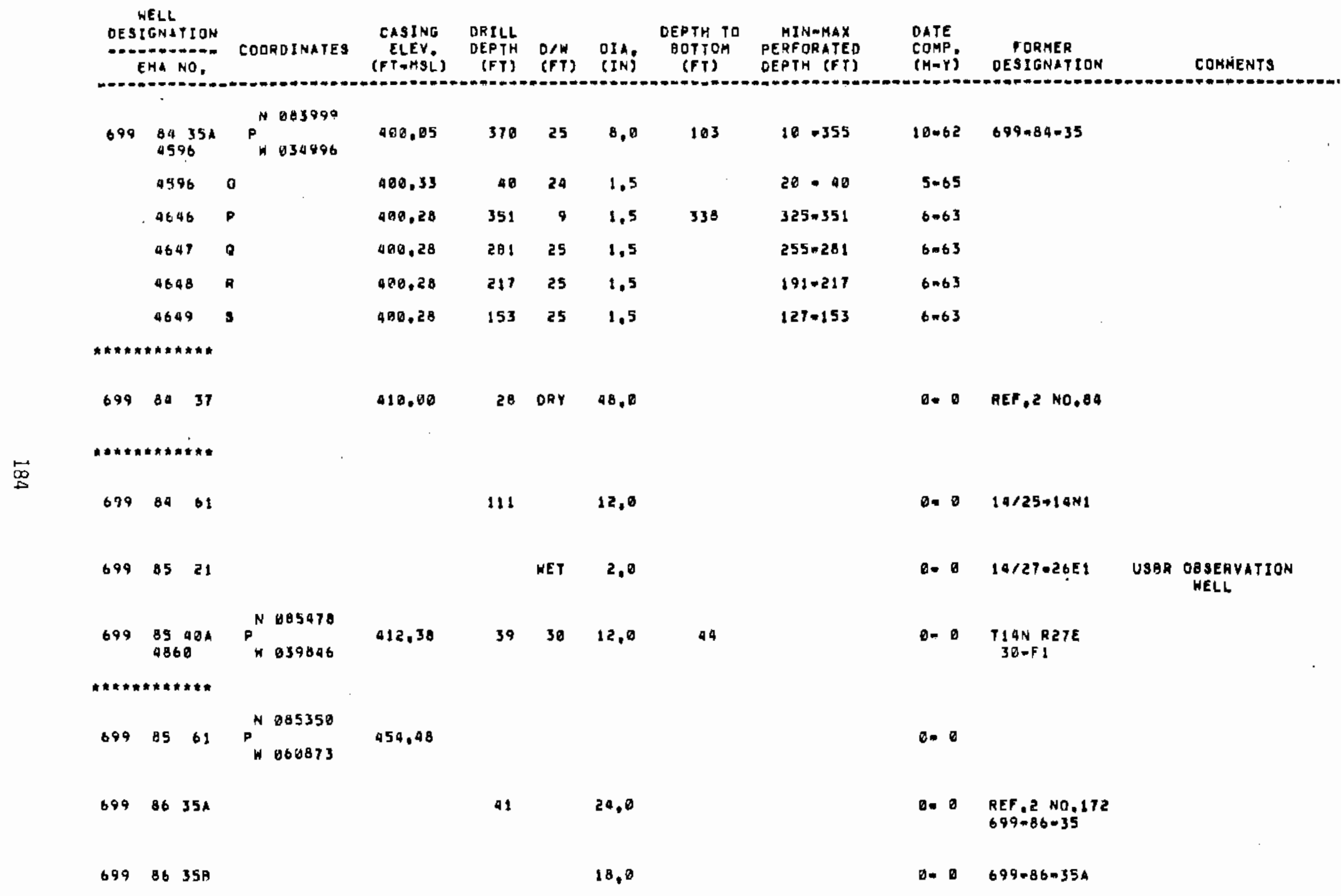




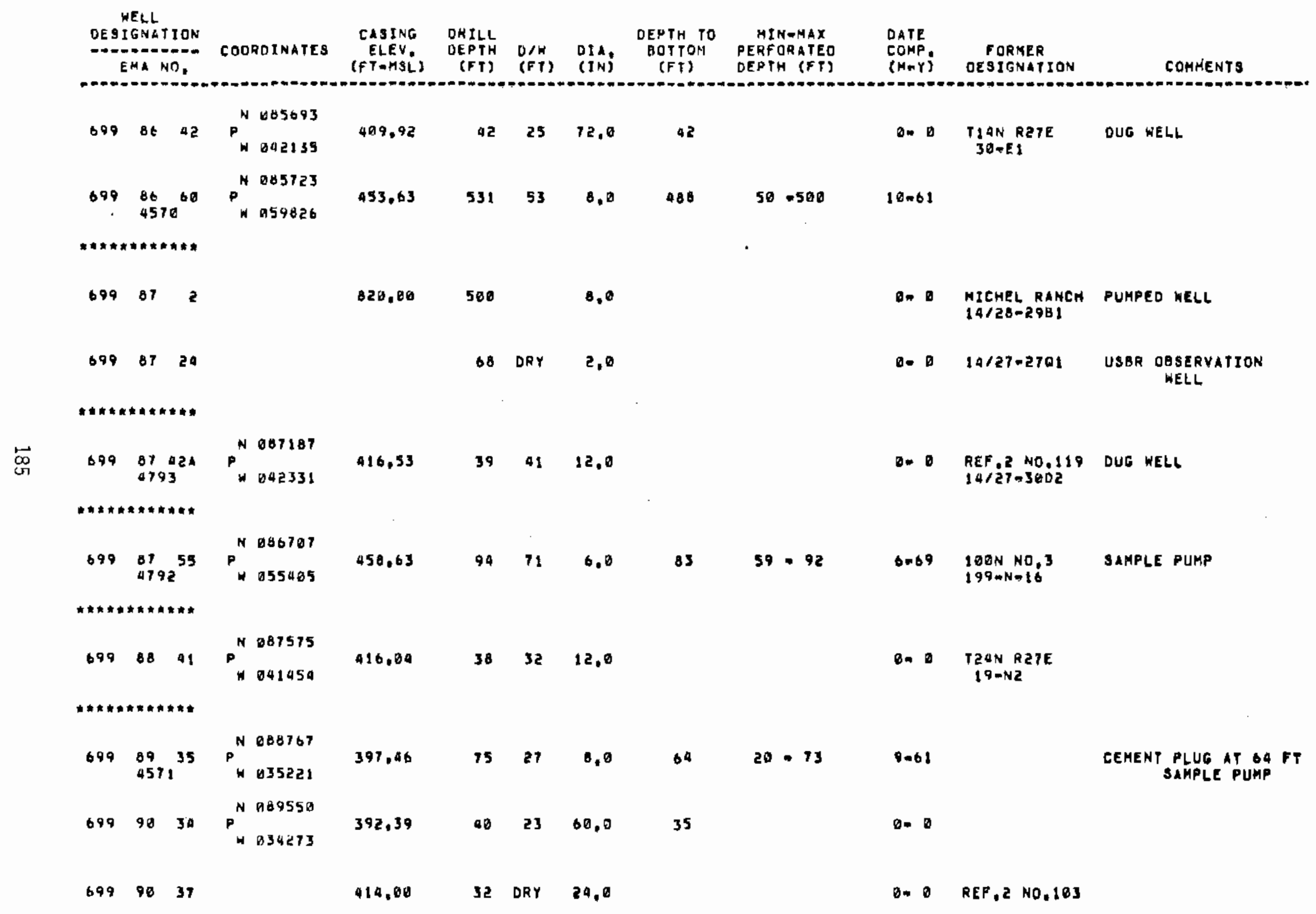




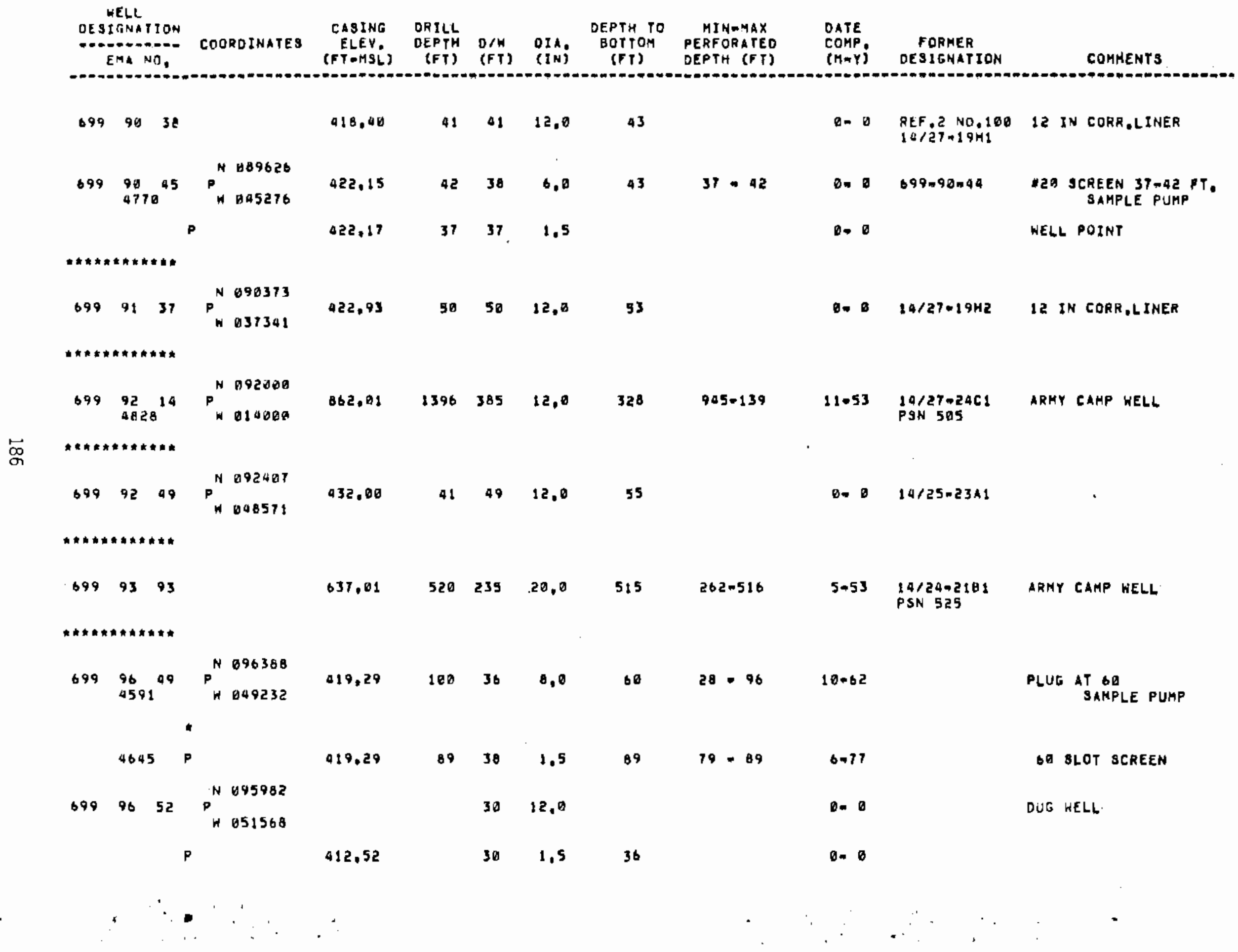




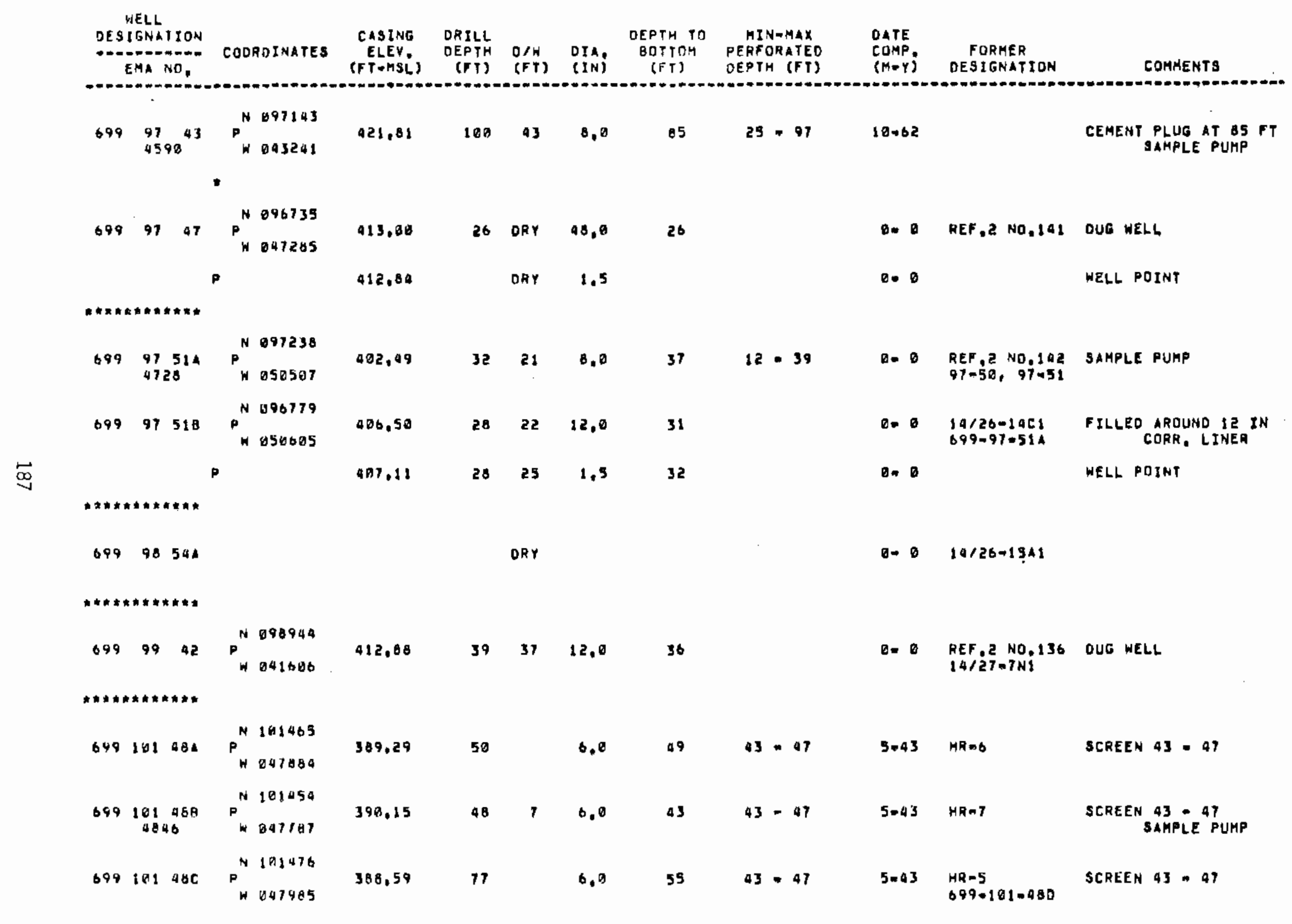




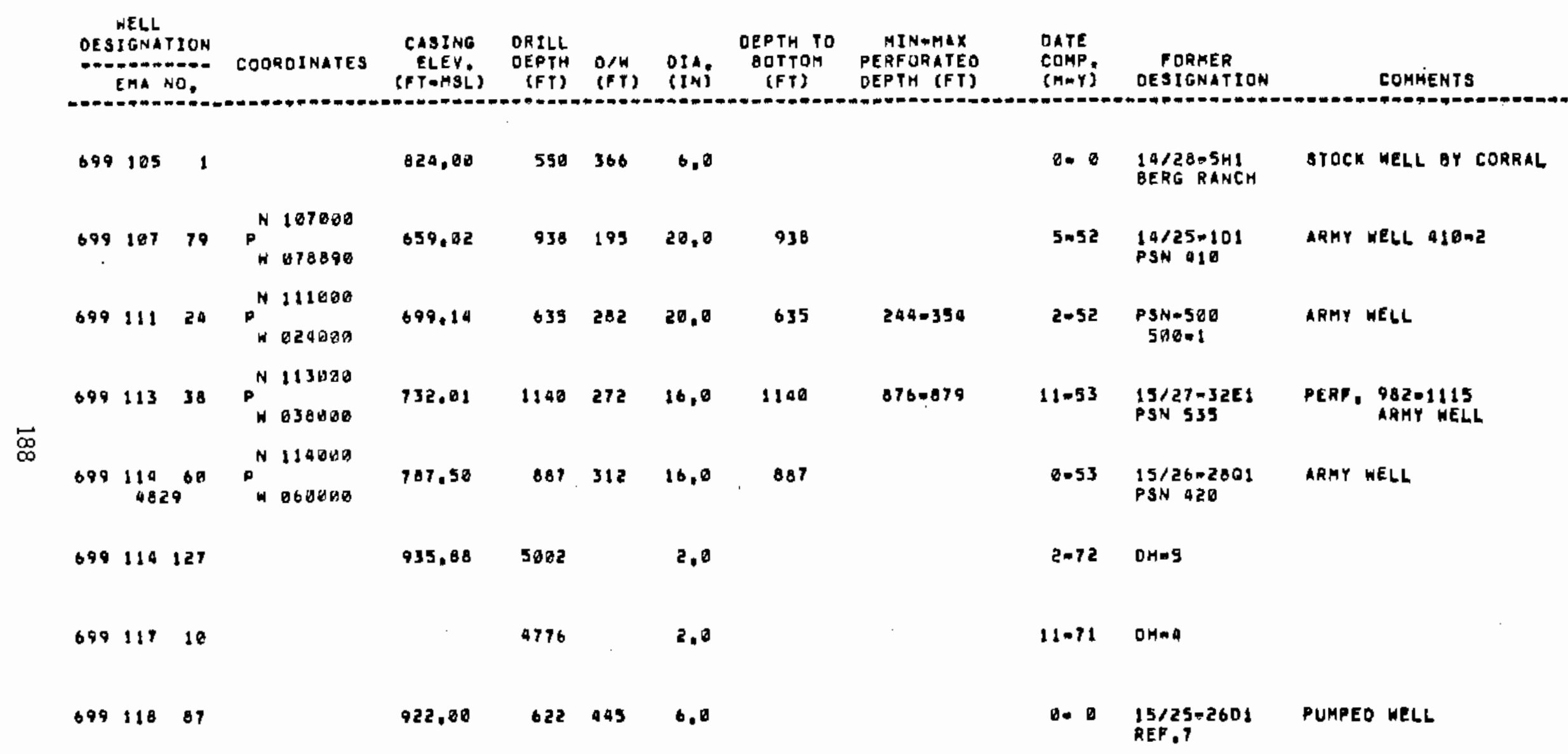


HELL

OESIGNATION

DESIGNATION

COORDINATES

CASING

ELEV.

DRILL (FTOKSL) (FT) (FT) (IN) (FT) DEPTH (FT)

BOTYOM

MINMMAX
PERFORATED

DATE

EMA NO.

COHP, FORHER

(HOY) DESIGNATION

COMHE्NTS

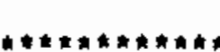




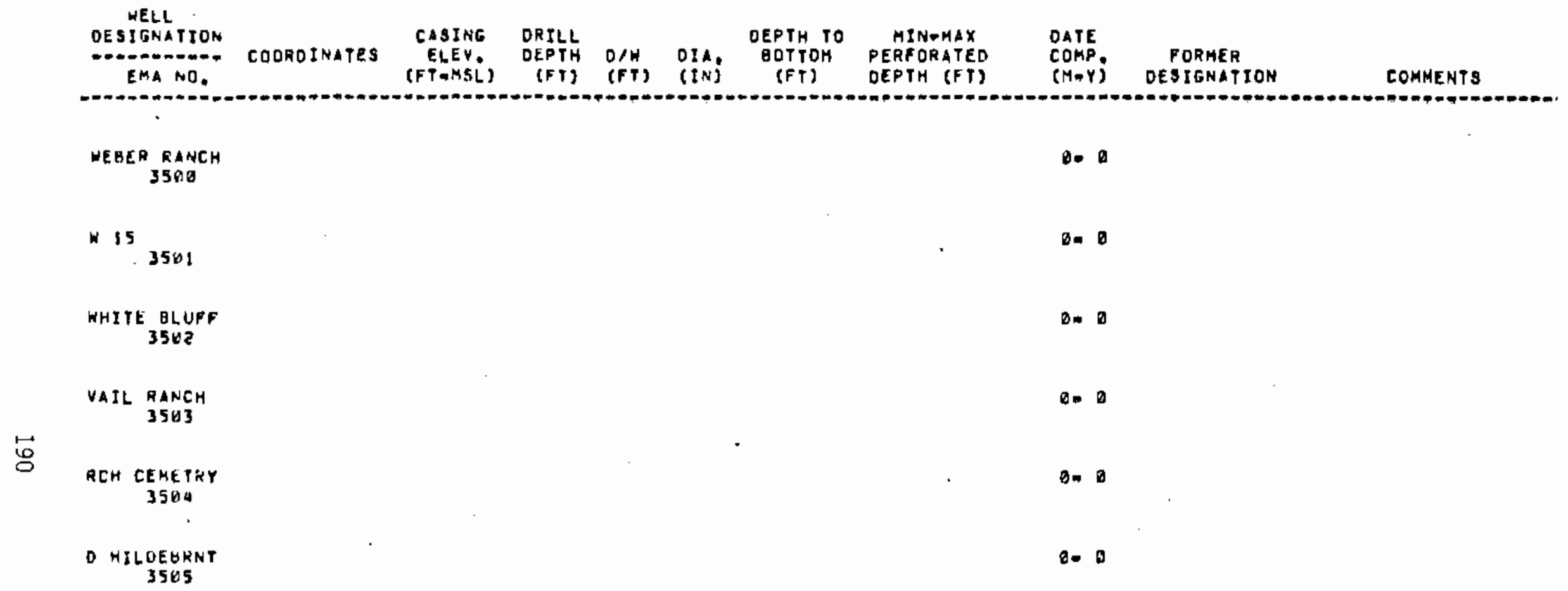




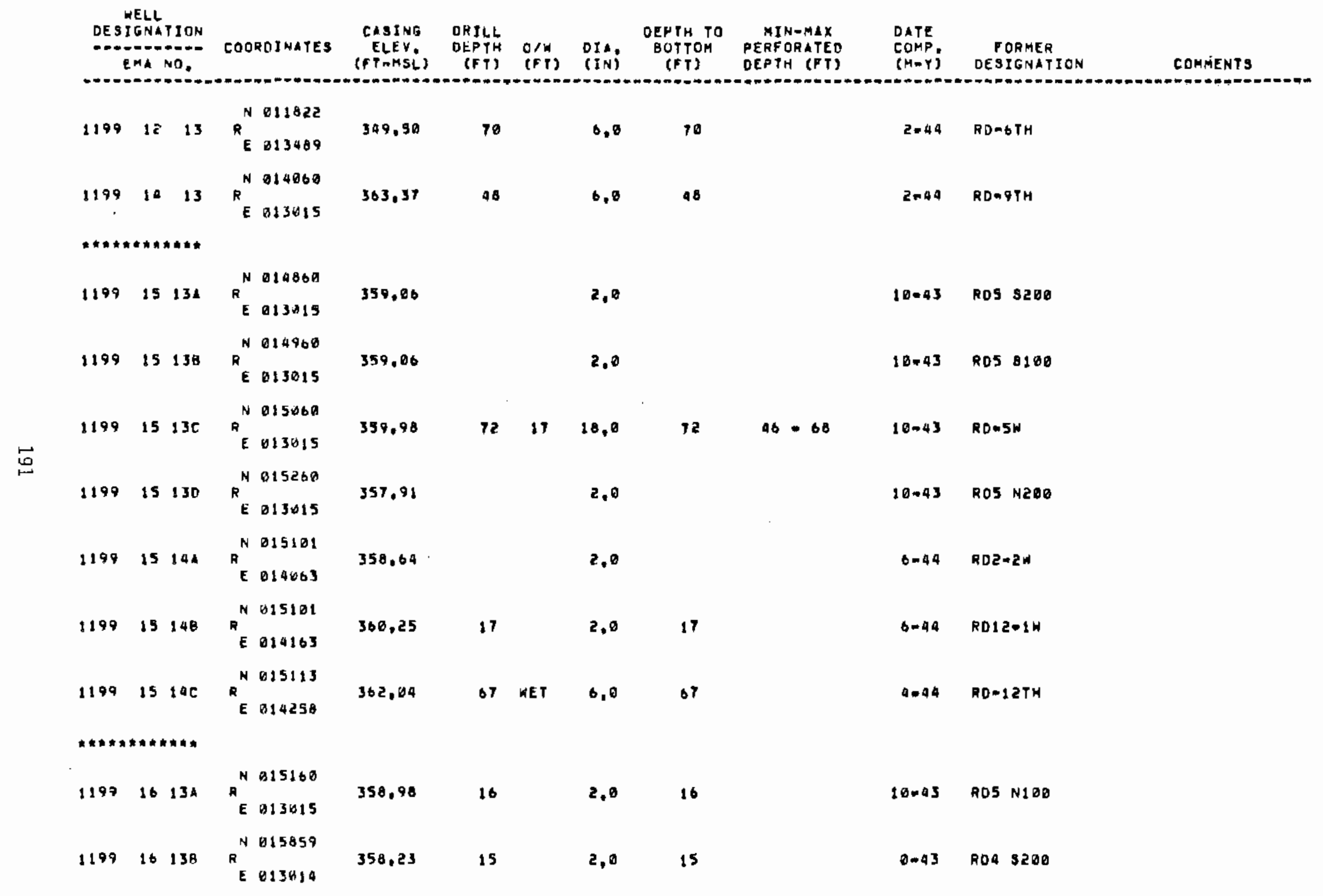




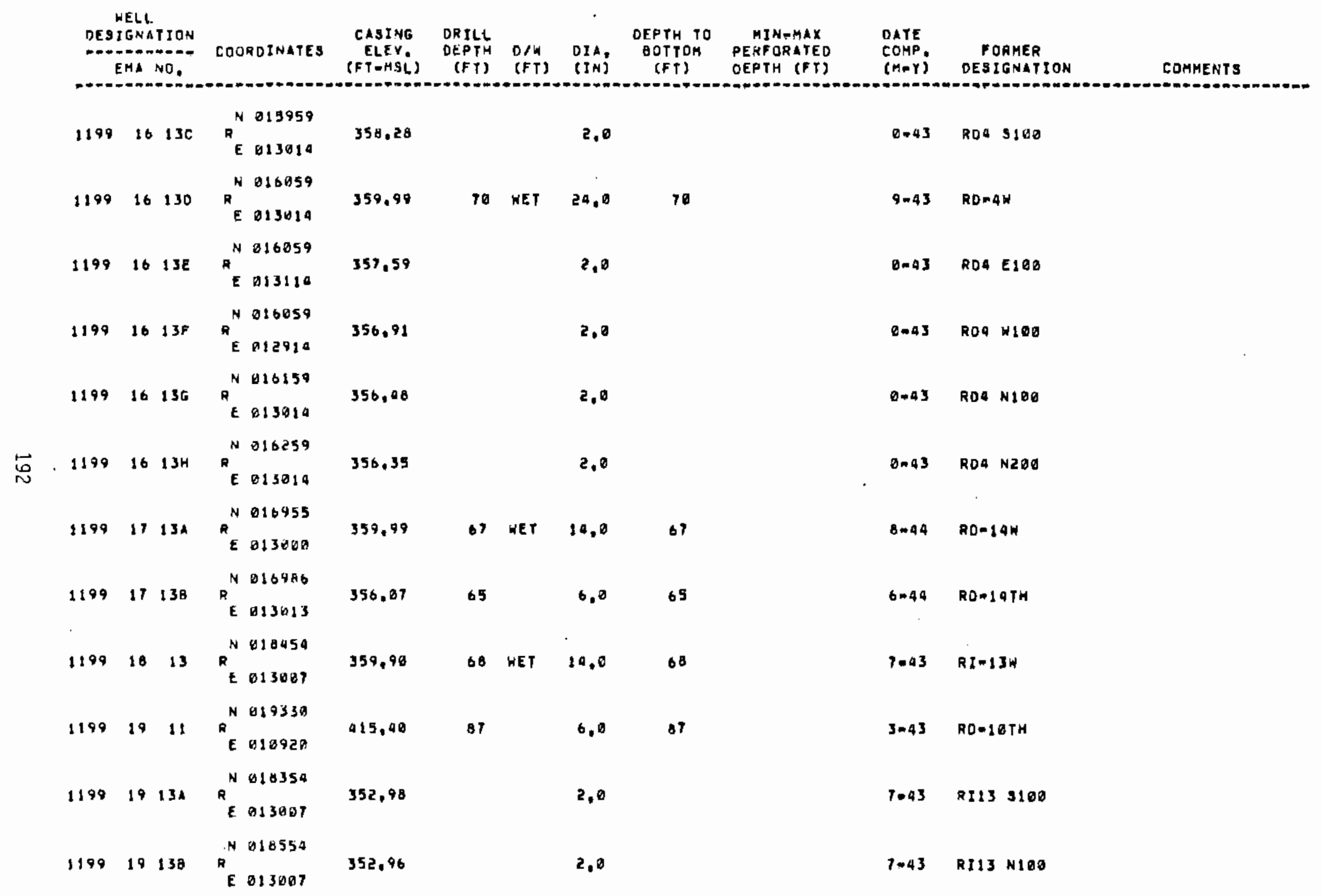




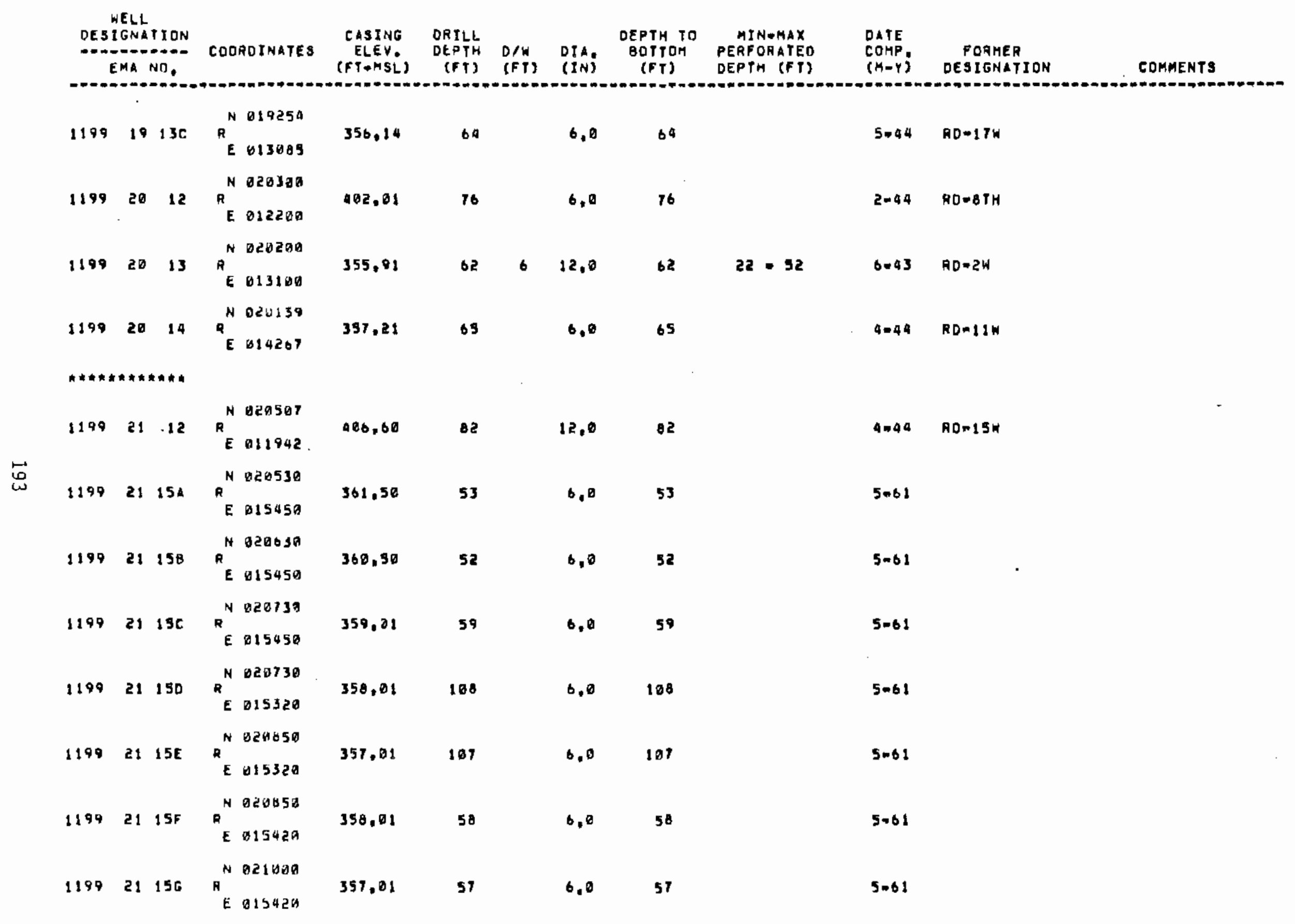




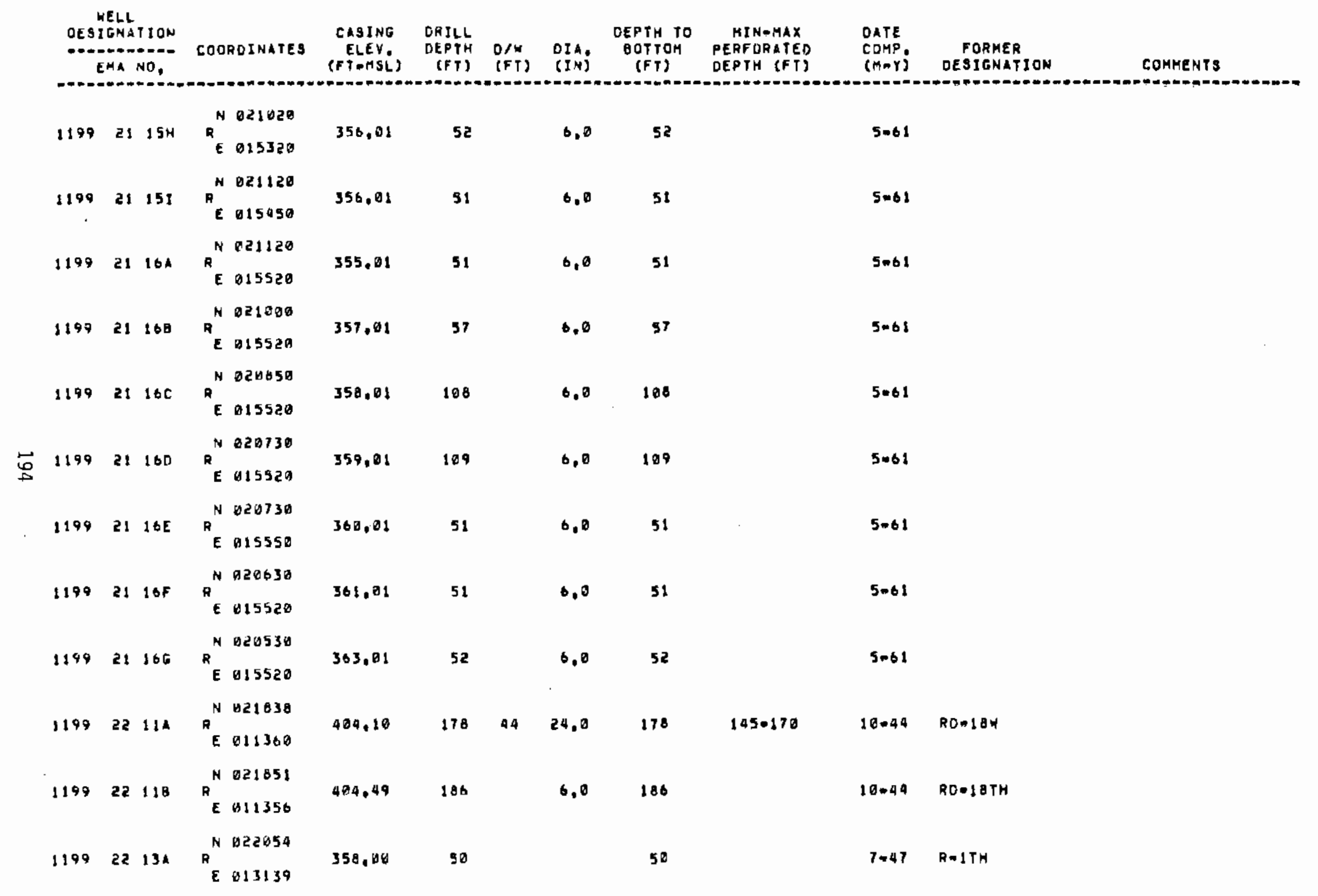




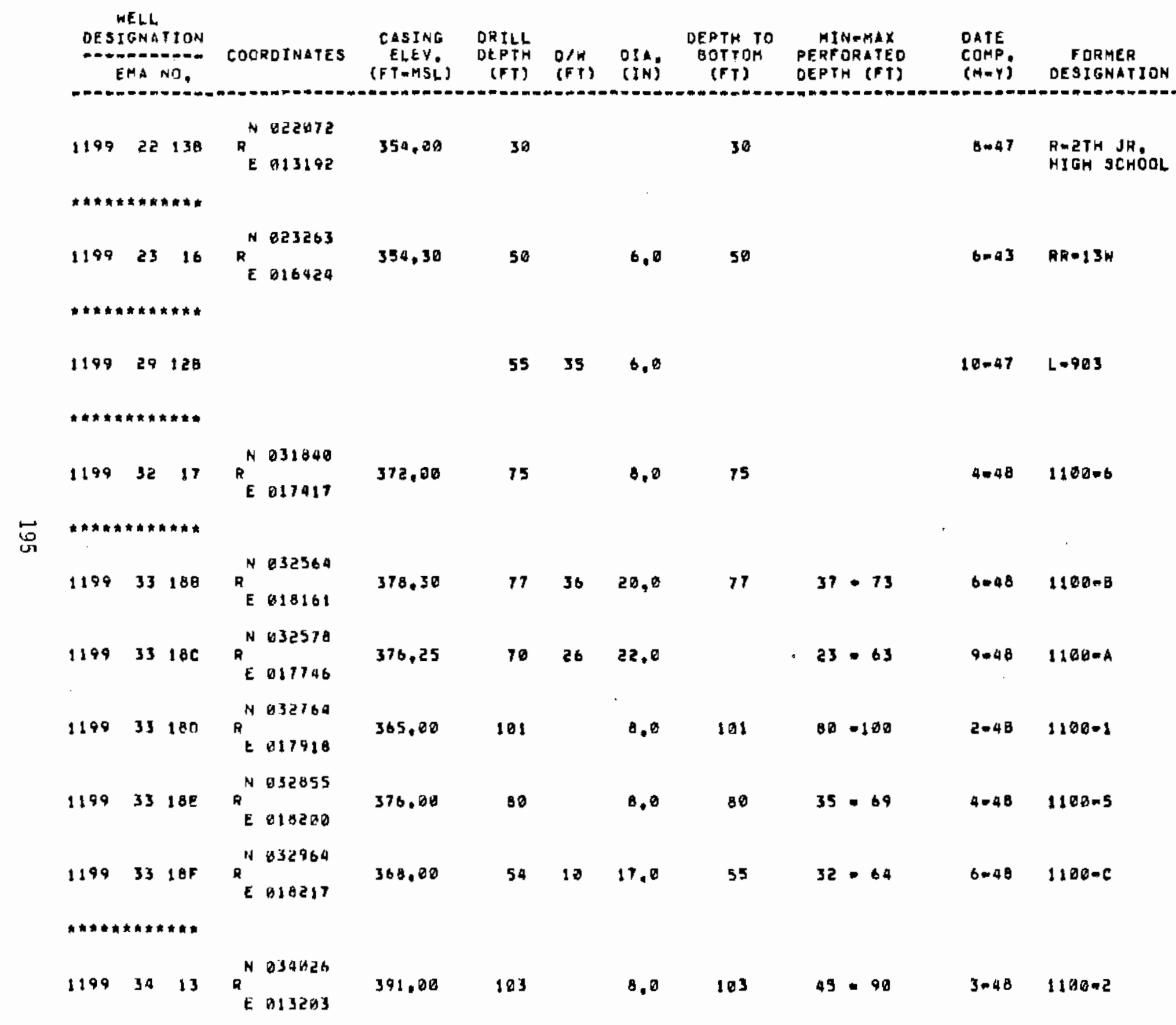




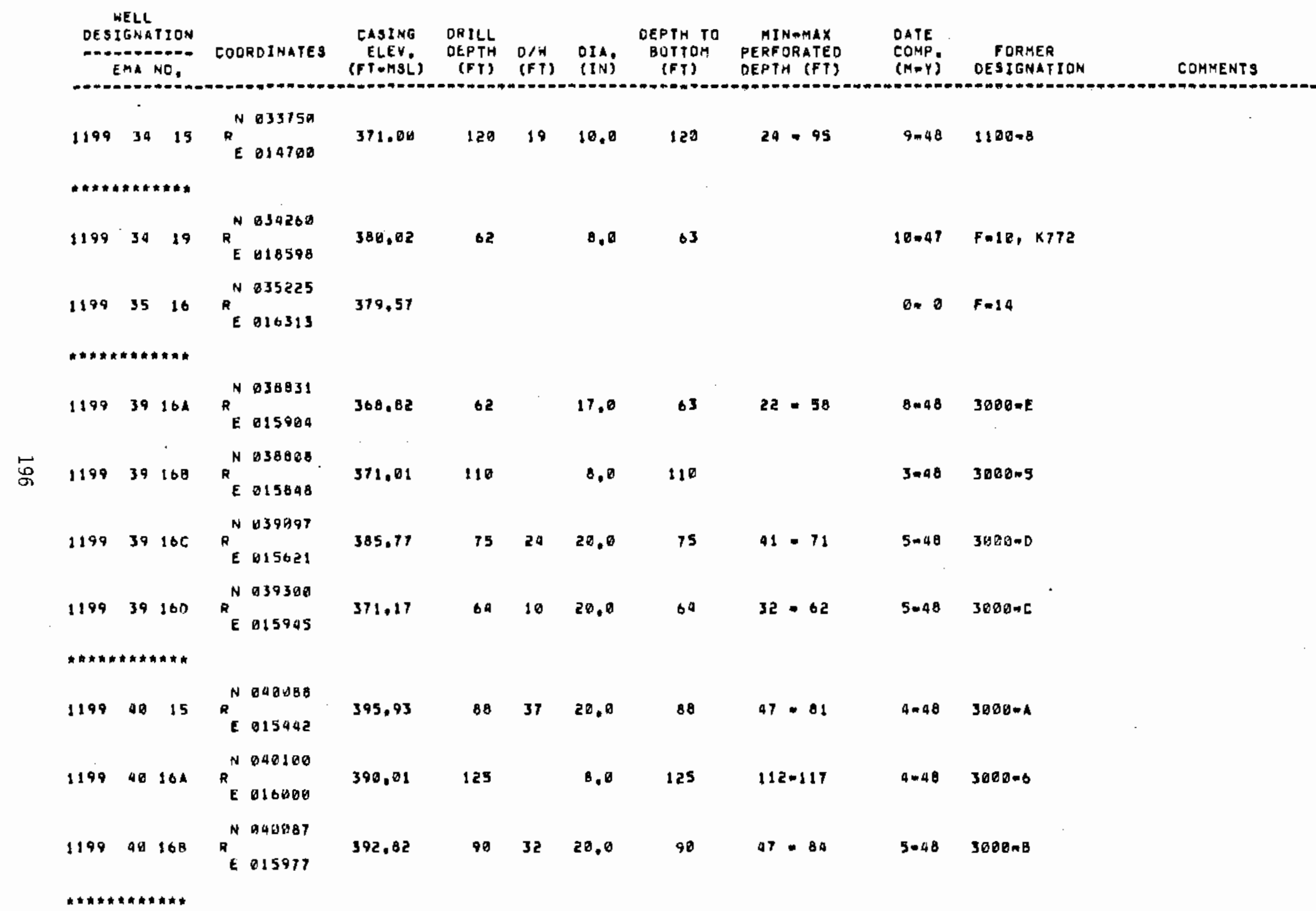


HELL

OESIGNATION

EMA

COOROINATES CASING DRTLL

DIA, DEPTH TO MITOM PERFORATEO

(FT) (FT) (IN) (FT) DEPTH (FT)

DATE

COMP, FORHER

(M-Y) DESIGNATION

COHHENTS

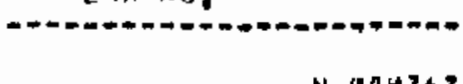

119

115

$R^{N 040767}$

398,94

74

17.0

74

$18-49$

3000- 6

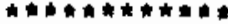




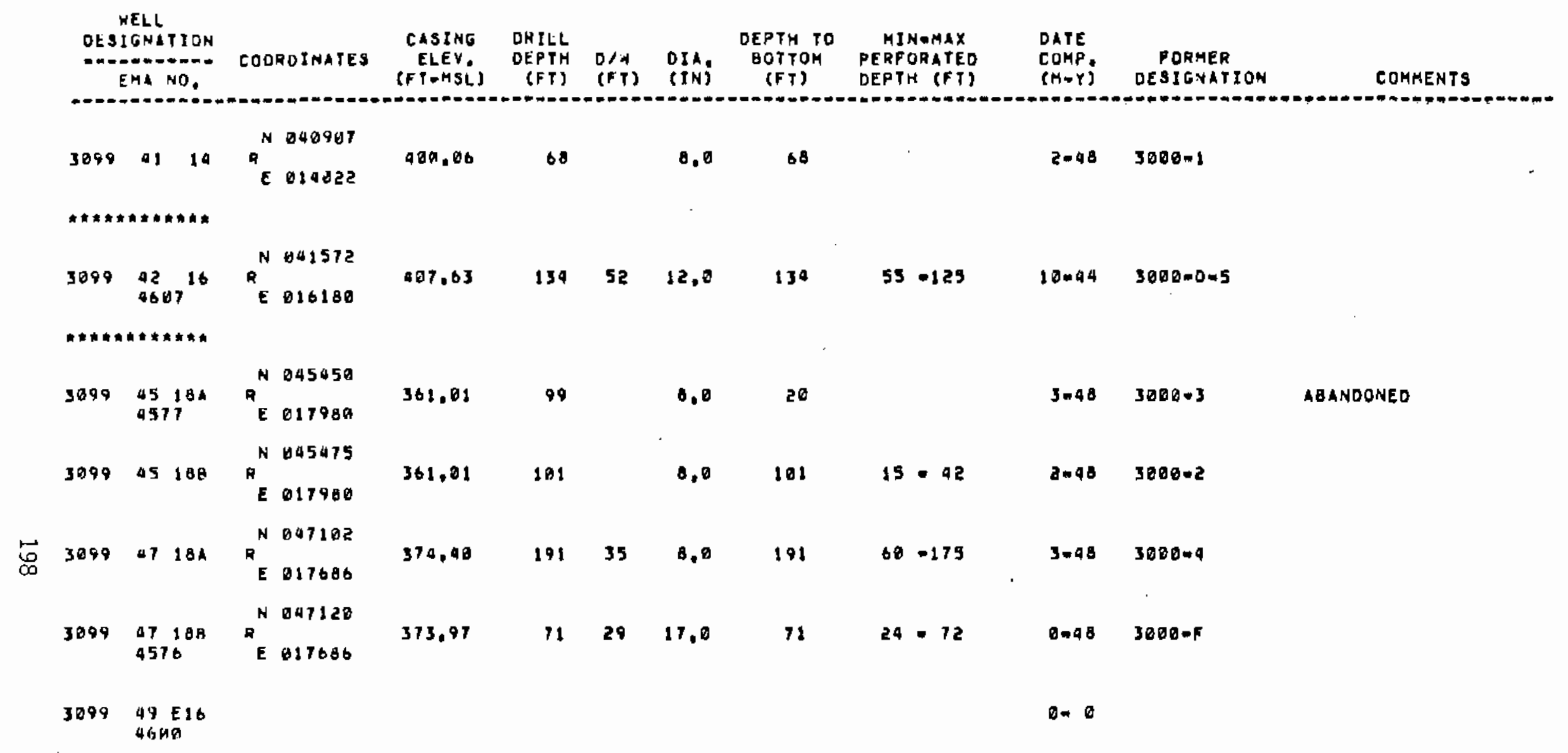




\section{DISTRIBUTION}

No. of

Copies

OFFSITE

$$
\begin{aligned}
& \text { A. A. Churm } \\
& \text { DOE Chicago Patent Attorney } \\
& 9800 \text { S. Cass Avenue } \\
& \text { Argonne, IL } 60439
\end{aligned}
$$

27 DOE Technical Information Center

\section{ONSITE}

7 DOE Richland Operations Office

D. R. Elle

D. E. Gerton

A. G. Lassila

H. E. Ransom

M. W. Tiernan

M. G. White (2)

\section{Rockwe 1] Hanford Operations}

M. K. Addition

H. Babad

R. L. Biggerstaff

L. E. Bruns

R. W. Bryce

D. L. Chase

R. A. Gephart

G. L. Hanson

K. K. Kover

A. G. Law

R. K. Ledgerwood

J. V. Panesko

W. H. Price

A. E. Rowlette

F. A. Spane

A. M. Tallman

G. L. Wagenaar

C. T. Webster

T. J. Wood
No. of

Copies

Hanford Environment Health Foundation

L. J. Maas

Vitro Engineering Corporation

S. S. Compton

3 Westinghouse Hanford Company

G. D. Carpenter

R. B. Hall

K. D. Hayden

2 United Nuclear Inc.

T. E. Dabrowski

E. A. Weakly

Pacific Northwest Laboratory

47 P. J. Blumer

P. E. Bramson

D. B. Cear lock

J. P. Corley

D. W. Damschen (5)

D. W. Dragnich

P. A. Eddy (2)

J. R. Eliason

E. L. Hilty

J. R. Houston

J. W. Lindberg

V. R. McGhan (10)

M. R. Miller

D. A. Myers

K. R. Price

M. R. Quarders (3)

J. R. Raymond

B. D. Robertson

C. M. Unruh

Publishing Coordination (2)

Technical Information (5)

Water and Land Resources Library (5)

Ro 


\section{100}

Wouring $10-100$

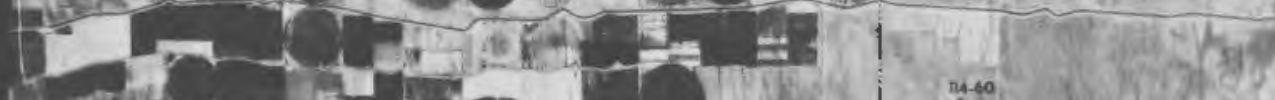

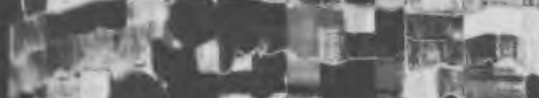

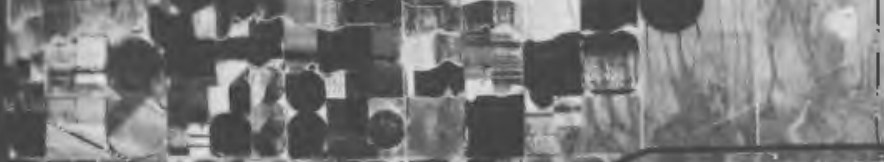

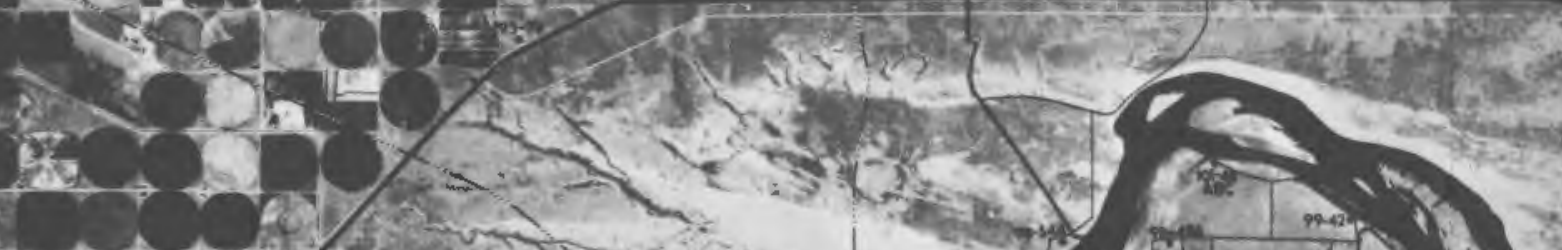

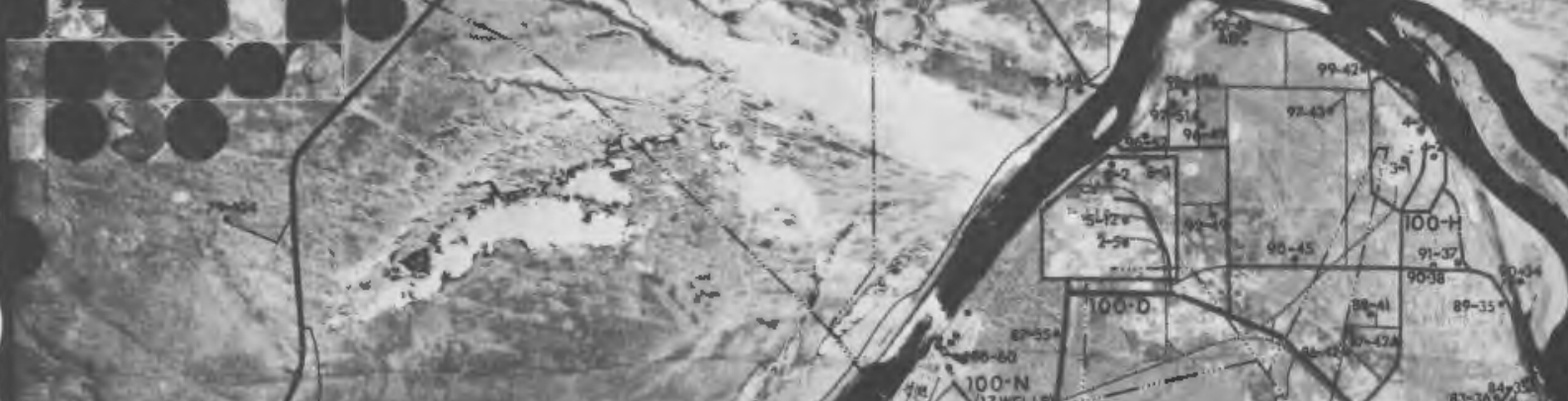

|

(15)

(1)

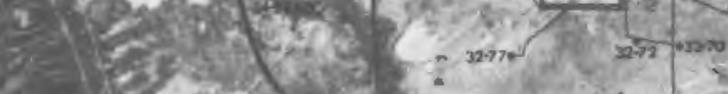

tras

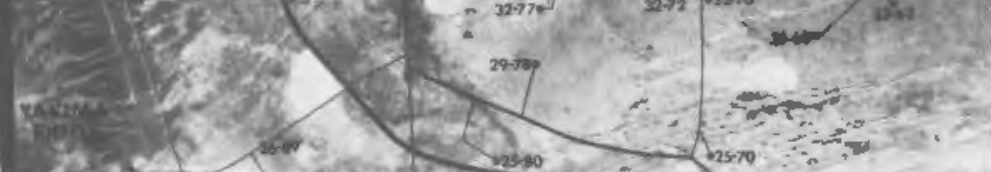

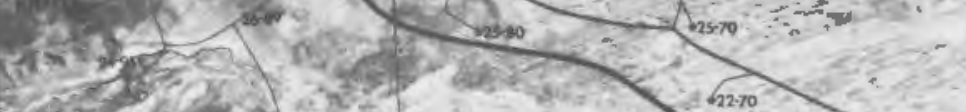

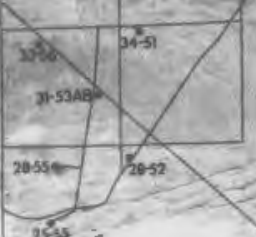

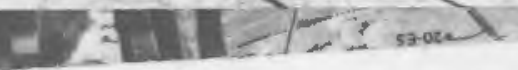


